\title{
CLARIFYING THE NATURE OF THE ASSOCIATION BETWEEN SOCIAL DOMINANCE ORIENTATION AND ENVIRONMENTALISM
}

\section{SAMANTHA KAY STANLEY}

\author{
A thesis \\ submitted to the Victoria University of Wellington \\ in fulfilment of the requirements for the degree of \\ Doctor of Philosophy
}

Victoria University of Wellington

(2019) 



\begin{abstract}
A small number of studies have reported a negative association between social dominance orientation (SDO: the relative preference for social inequality and intergroup dominance) and environmentalism. However, the existing research has yet to fully investigate the nature of this relationship. In this thesis, I develop a programme of research that aims to clarify how ideology relates to environmentalism, in several important ways.

I start by systematically reviewing the relationship between SDO, the related ideological construct of right-wing authoritarianism (RWA: the preference to submit to authority and tradition, and punish those who deviate), and environmentalism. I do this by meta-analysing the associations reported in the existing literature, and previously unpublished datasets. These analyses show that both ideological constructs are important for understanding human-environment relations. Importantly, SDO is more strongly related to environmentalism in general population samples than in student samples, which helps to reconcile previous inconsistencies in the literature.
\end{abstract}

I then explore the ideology-environmentalism link over time, comparing the strengths of the associations with environmentalism and SDO and RWA. Consistent with the metaanalyses I show that, while RWA is more predictive of changes in environmental attitudes over time in student populations, SDO is the key predictor among general population samples. Therefore, dependent on sample, these findings indicate that endorsing these ideologies lead to changes in environmentalism over time.

Next, I elaborate on the SDO-environmentalism relationship by exploring whether and how the two facets of SDO (anti-egalitarianism and dominance) differentially relate to environment-relevant attitudes. Overall, I find that anti-environmental attitudes are largely driven by individual preference for inequality rather than for intergroup dominance.

Previous work, including my own, has focused exclusively on quantitative surveybased methods. I invited individuals scoring relatively high, moderately, and low on SDO to share their ideas on climate change. Interviews with these individuals revealed that many were concerned about the causes, consequences, and potential solutions to climate change. Most participants were also armed with justifications excusing their, and others', inaction on the problem. To establish how the ideas shared in the interviews related to SDO, I reworked them into statements for survey-based research. This research demonstrates that ideology (both SDO and RWA) also related to most of these interview-based statements, with those 
scoring higher on dominance attitudes more opposed to top-down action on climate change, and those more tolerant of inequality more opposed to individual action.

In sum, my work adds to the growing body of research that establishes ideology as a barrier to environmental engagement. My findings support the interpretation of SDO as a barrier to engagement through an environmental justice framework. If we are to stop the warming of the planet and increased social inequality that this brings, then we must also understand ideology as a barrier to belief and action on climate change. 


\section{Preface}

What impact does a single individual have on the planet? Each of us is so small, and the world and its resources are so vast. If we are to make a change for the better, such as giving up meat, forgoing driving, or taking the time to sort our recyclables, it may have only a minute impact on the global scale, given that we share the planet with over seven billion others. I appreciate these attitudes, and how they make pro-environmental action seem futile. However, I also believe that every decision we make has an effect on the environment.

That effect can be positive or negative and, whether positive or negative, and regardless of its size, these effects are also cumulative. Each may just be a 'small drop in the bucket', but they all drop into the same bucket. Choosing environmentally harmful behaviours leads to increases in atmospheric levels of $\mathrm{CO}_{2}$ and depletes natural resources. Choosing them repeatedly, and on a larger scale, multiplies these effects. Our choices contribute to the environmental problems that we are currently experiencing - problems that are expected to get worse.

Because our actions affect the planet, they also affect its other inhabitants. Climate change is increasingly being acknowledged as a social justice issue (Agyeman, Schlosberg, Craven \& Matthews, 2016; Schlosberg \& Collins, 2014). Individuals and industries in some countries pollute our planet at an ever-increasing rate. Yet there are others who live within the bounds of what the natural environment can support, leaving little more than footprints. They are also the ones most threatened by rising sea levels, scarce resources interrupting their way of life, and more devastating effects of our choices.

Demands to reduce our impact on the planet are becoming more frequent and more desperate. We see this in IPCC reports, discussions at international summits in Kyoto, Copenhagen and Paris, open letters from scientists, and in the next generation walking out of school to strike for action (IPCC, 2014; Ripple et al., 2017; United Nations, 2015). Climate change will impact standards of living, and this is especially the case for those at the bottom of the social hierarchy. But we require those at the top to make sacrifices now. This places action on climate change at the intersection of environmental and social responsibility.

To me, both of these things are important, but to some, neither are. That really fascinates me - enough to spend three years studying it, and hopefully many more. The research in this thesis is not going to solve this problem by any stretch. But for me, it was a helpful start at understanding individual differences in attitudes towards these issues. 


\section{Acknowledgements}

I am most grateful to my supervisor, Professor Marc Wilson, for his advice, encouragement, and expertise. I must also acknowledge Taciano Milfont, who was part of my supervisory team for the studies presented in Chapters 3 and 4, and the design of the interview schedule in Chapter 5. I am grateful to have received the Victoria Master's by thesis scholarship, which supported the first year of my studies, before upgrading to a $\mathrm{PhD}$ with the support of a Victoria Doctoral Scholarship.

Finally, I feel incredibly lucky to have had the support of a great group of friends at Victoria, as well as my mum and my partner Nathaniel, who read many drafts and provided endless encouragement. 


\section{Publications}

Some of the work included in this thesis formed the basis of publications. These were published with joint authorship, therefore in this section I outline the extent of my contribution to each study. In all cases, where my co-authors made changes to my writing for the published version of this work, these changes were not retained in my thesis chapter, such that the writing included in my thesis is my own.

Study 1A and 1B (Chapter 2) form the basis of the publication Stanley and Wilson (2019). Marc Wilson originally had the idea for Study 1A, and I had the idea for Study 1B. I did the literature search, contacted authors in this area for additional work to include, and conducted all analyses. I was also responsible for interpreting the analyses and the writing of the manuscript and Chapter.

The studies in Chapter 3 used data collected by Marc Wilson (Study 2A; Stanley, Wilson \& Milfont, 2017a) and Chris Sibley (Study 2B; Stanley, Milfont, Wilson \& Sibley, 2019). For these studies, I conducted the analyses, interpreted results, and wrote the first draft of the manuscript. Where my co-authors made changes for the publication, I included my own writing in my thesis.

I had the idea for Study 3A, 3B, and 3C (Chapter 4; Stanley, Wilson, Milfont \& Sibley, 2017b). Data for Study 3A were from Chris Sibley, I collected the data for Study 3B, and Study $3 \mathrm{C}$ relied on an existing dataset from Marc Wilson. I did all analyses, data interpretation, the first complete draft of the manuscript, and all writing of this Chapter.

Study 4 (Chapter 5) is unpublished, though will likely be reworked and submitted for publication as a co-authored piece with Taciano Milfont and Marc Wilson. Milfont and Wilson assisted in the conceptualization and refining of the research idea, as well as refining the interview schedule. I did all data transcription, thematic analysis, and writing of this chapter. I had the idea for Study 5A and 5B (Chapter 6, unpublished), and developed the items with feedback from Marc Wilson. I also did all aspects of data collection, analysis, interpretation, and the writing of this chapter.

The Chapters that frame the published work (General Introduction/Chapter 1 and General Discussion/Chapter 7) are my own writing, and have not formed the basis of publications. 


\section{Contents}

Page

Abstract I

Chapter 1: General Introduction

Background 1

$\begin{array}{ll}\text { Literature review } & 7\end{array}$

Chapter 2: Ideology and environmentalism: A meta-analysis

Introduction to studies 1A and 1B 21

Study 1A: Social dominance orientation and authoritarianism: 24

Meta-analysing association between ideology and

environmentalism

Study 1B: Meta-analyses of the unique effects of ideology on $\quad 51$

environmentalismDiscussion of Studies $1 \mathrm{~A}$ and $1 \mathrm{~B}$

Discussion of studies $1 \mathrm{~A}$ and $1 \mathrm{~B}$

Chapter 3: Examining the associations between ideology and environmentalism over time

Introduction to Studies 2A and 2B 67

Study 2A: Exploring short-term longitudinal effects of social 69

dominance orientation and right-wing authoritarianism on

environmentalism

Study 2B: The influence of social dominance orientation and right-wing authoritarianism on environmentalism: A five-year cross-lagged analysis

Discussion of Studies 2A and 2B

Chapter 4: Dimensions of social dominance and their associations with environmentalism

Introduction to Studies 3A, 3B and 3C

99

Study 3A: Separating the dimensions of social dominance orientation to predict environmentalism in a general population sample

Study 3B: Examining how the two dimensions of social dominance orientation differentiate to predict environmentalism using the $\mathrm{SDO}_{7}$

Study 3C: Longitudinal relation between the dimensions of SDO and environmentalism

Discussion of Studies 3A, 3B and 3C 
Chapter 5: Examining the discourses of high-, moderate-, and lowSocial Dominants' surrounding environmental issues

Study 4: Thematic analysis of semi-structured interviews about climate change with individual's scoring low, moderate, and high in social dominance orientation

Chapter 6: Understanding how student's ideas about climate change relate to endorsement of SDO

Introduction to Studies 5A and 5B 213

Study 5A: Pilot study: Testing quantitative measures of the 216 ideas expressed in the interview study about climate change

Study 5B: How does social dominance orientation relate to student's ideas about climate change?

Discussion of Studies 5A and 5B

$\begin{array}{lll}\text { Chapter 7: } & \text { General discussion } & 267\end{array}$

$\begin{array}{ll}\text { References } & 283\end{array}$

$\begin{array}{ll}\text { Appendices } & 308\end{array}$ 


\section{List of Tables}

$\begin{array}{lll}\text { Table Title Page } & \end{array}$

Table 2.1 Coding schedule used to categorize environmental variables. 34

Table 2.2 Results of the meta-analyses of the correlation between 46 $S D O, R W A$, and six environment-relevant variables.

Table 2.3 Results of the meta-analyses of regression coefficients 60 between SDO, RWA, and four environment-relevant variables.

Table 3.1 Descriptive statistics, and correlation matrix between mean scores of each variable

Table 3.2 Descriptive statistics and correlations between variables across all time points.

Table 4.1 Model fit for one- and two-dimension models, two-method models, and four-factor models used in each study presented in Chapter 4.

Table 4.2 Descriptive statistics, and correlation matrix between mean scores of each variable in Study $3 A$.

Table 4.3 Wald test results comparing significance of SDO-D and SDO-E as predictors of environment-relevant variables.

Table 4.4 Descriptive statistics, and correlation matrix between mean scores of each variable in Study $3 B$.

Table 4.5 Descriptive statistics, and correlation matrix between mean scores of each variable in Study 3C.

Table 5.1 Descriptive information about the SDO groups from preselection data for Study 4.

Table 5.2 Descriptive information about the SDO groups from those 
interviewed in Study 4.

Table 5.3 Codes included in the causes and consequences of climate change theme.

Table 5.4 Codes included in the hopeless environmentalist theme.

Table 5.5 Codes included in the solutions theme.

Table 5.6 Associations between SDO and the codes developed in Study 203 4.

Table 6.1 Descriptive statistics for the Likert response items, and their 228 associations with SDO in Study 5A.

Table 6.2 Descriptive statistics of sliding scale responses and their associations with social desirability and SDO in Study 5A.

Table 6.3 Descriptive information and correlations between mean scores in Study $5 B$.

Table 6.4 Rotated factor matrix.

Table 6.5 Descriptive information and correlations between environmentalism scale factors and SDO.

Table 6.6 Descriptive analyses of Likert-scale response items, including associations with SDO in Study 5B.

Table 6.7 Descriptive analyses of sliding scale response set items, including associations with SDO in Study 5B.

Table 6.8 Descriptive information about the three groups based on SDO level in Study $5 B$.

Table 6.9 Group-based differences in sliding scale responses. 


\section{List of Figures}

$\begin{array}{lll}\text { Figure Heading } & \text { Page }\end{array}$

Figure 2.1 PRISMA summary of studies included in meta-analyses of 32 zero-order correlations.

Figure 2.2 Mean meta-analytic correlation between ideological variables and environmental variables.

Figure 2.3 Funnel plot showing the distribution of studies and effect sizes for the meta-analysis between SDO and environmentalism.

Figure 2.4 Funnel plot showing the distribution of studies and effect sizes for the meta-analysis between RWA and environmentalism.

Figure 2.5 Partitioning of the variance explained in a multiple regression analysis, where environmentalism is regressed onto SDO and RWA.

Figure 2.6 Results of meta-analyses between SDO and RWA, using different sample types and measurement scales.

Figure 2.7 Meta-analysed regression coefficients on the associations between SDO, RWA, and several indices of environmentalism.

Figure 3.1 Standardized associations between SDO, RWA, NEP, and 76 climate change denial across time.

Figure 3.2 Unstandardized parameters assessing the association between SDO, RWA, and willingness to sacrifice for the environment.

Figure 3.3 Standardized parameters assessing the association between SDO, RWA, and willingness to sacrifice for the environment 
(WILL).

Figure 4.1 SDO-D and SDO-E predicting environmental variables in 110 Study 3 A.

Figure 4.2 SDO-D and SDO-E predicting environmental variables in 119 Study 3B.

Figure 4.3 Time one SDO-D and SDO-E predicting environmental variables concurrently in Study 3C.

Figure 4.4 Time two SDO-D and SDO-E predicting environmental variables concurrently in Study 3C.

Figure 4.5 Longitudinal associations between SDO-D and SDO-E and environmentalism in Study 3C.

Figure 4.6 SDO-D and SDO-E at Time 1 predicting environmentalism 128 at Time 2 in Study 3C.

Figure 4.7 Environmental variables at Time 1 predicting SDO-D and 129 SDO-E at Time 2 in Study 3C.

Figure 5.1 Thematic map of codes and themes in Study 4.

Figure 6.1 Example sliding scale item.

Figure 6.2 Mean differences between high- and low-SDO groups on the sliding scale items in Study 5A.

Figure 6.3 Placement on sliding scale by SDO group in Study 5B. 


\section{List of appendices.}

Appendix A Table A.

Summary of studies included in the meta-analyses of zeroorder correlations (Study 1A).

Appendix B Table B.

Summary of studies included in our meta-analyses of standardized regression weights.

Appendix C Replication of Study 3B and 3C using shortened $\mathrm{SDO}_{7}$ scale.

Appendix D Interview schedule used in Study 4. 


\section{Clarifying the nature of the association between social dominance orientation and environmentalism}

\section{Chapter 1: General introduction}

"Climate change represents an urgent and potentially irreversible threat to human societies and the planet"

United Nations (2015, p. 2).

\section{Background}

The purpose of my thesis is to try to understand why people hold particular attitudes about the environment, not to review and debate the science behind climate change, or to convince my readers that it is real. However, here I briefly describe what the problem is, and include some citations for the interested reader.

Put simply, the climate of a region is its average weather, and the climate is described as changing when there is a shift in either the mean or the variability in the climate's properties (IPCC, 2014a). In the past three decades, we have experienced significant changes to the global climate, with each decade growing warmer than the last (IPCC, 2014a). The Intergovernmental Panel on Climate Change (IPCC, 2014a), which produces reports by over 2500 contributing climate scientists, link these recent changes to the increase in greenhouse gas emissions from human activities following the Industrial Revolution.

Although changes in climate can and do result from natural variability, there is near unanimous agreement that recent changes are attributable to human actions (Carlton et al., 2015; Cook et al., 2016; Verheggen et al., 2014). These changes include global surface temperatures rising at a rapidly increasing pace, extreme weather events such as heavy rain and heat waves becoming more common, and the reduction in arctic sea ice and corresponding rise in sea level (IPCC, 2014a).

Another IPCC working group (Collins et al., 2013) has predicted that at the current rate, we are likely to see an increase in the global temperature of between 1.4 and $5.8^{\circ} \mathrm{C}$. Relating to this, the majority of coastlines will experience advancing shorelines, and natural 
systems will be disrupted. An example is the water cycle, where some areas will experience drastic increases, while others a reduction, in precipitation levels. These effects have obvious implications for low-lying coastal areas, but also for crop production, which relies on rain and certain temperatures to thrive, water resources, and human health (IPCC, 2014b).

Burning of fossil fuels, increasing animal agriculture, and deforestation all contribute to greenhouse gas emissions: chemicals such as carbon dioxide, nitrous oxide and methane that build in concentration and work to trap heat in the atmosphere (e.g. Chiles, Fabian, Tobin, Colby \& DePue, 2018; de Sousa-Neto, Gomes, Nascimento, Pacheco \& Ometto, 2018; Shurpali, Agarwal \& Srivastava, 2019). Growth of the population and economy put a greater strain on the environment. Humans already take more natural resources from the environment than is sustainable, and with more people on Earth, this only works to increase that burden (Casey \& Galor, 2017; Guillebaud \& Hayes, 2008; IPCC, 2014a; Scovronick et al., 2017). The consequence of this is that, each year, the date that humans take more resources than the planet can supply (Earth Overshoot Day) grows closer to the start of the year. In 2017, we had exhausted resources and exceeded the planet's ability to regenerate these resources by August $2^{\text {nd }}$, when in 2013 we lasted until the $20^{\text {th }}$ of August (Global footprint network, 2018; Posthuma et al., 2014). Last year, Earth Overshoot Day was August $1^{\text {st }}$ (Global footprint network, 2018).

At this point, it is almost inevitable that we will experience two degrees Celsius of warming, with action to mitigate the damage unlikely to reverse this (IPCC, 2014a). Stopping or slowing the rate of climate change requires action from countries, corporations, and individuals to reduce their greenhouse gas emissions. Despite this, people tend to view themselves and those close to them as less vulnerable to climate change. Specifically, people are least likely to see climate change as a threat to them personally, a little more likely to imagine those in their own country being affected, though those in developing countries and future generations are perceived as more likely to be affected (Leiserowitz, Maibach, RoserRenouf, Feinberg \& Rosenthal, 2015; Leiserowitz et al., 2017). This is because people are inclined to view climate change as psychologically distant, with themselves, and people like themselves, more immune to the effects. The effects are not only considered uncertain, but imagined as far away both geographically and in time (Spence, Poortinga \& Pidgeon, 2012).

In truth, these perceptions may be partly correct. While humans are causing the rapid heating of the planet, those contributing most to climate change, and those most vulnerable to 
its effects, are not necessarily the same people. The IPCC (2014b) note that hazards associated with climate change are likely to exacerbate stressors and consequently effect people's livelihoods, but these effects are not unilateral. Instead, the poor will be most affected, and least able to afford to adapt to the effects of climate change. Those in developing countries with low incomes are also most likely to experience negative consequences of climate change on their health (Althor, Watson \& Fuller, 2016).

Instead, the University of Notre Dame Global Adaptation Initiative (UND-GAI; Chen et al., 2015), which indexes vulnerability and resilience to the effects of climate change, ranks several affluent countries - those contributing the most to global emissions - as most likely to survive climate change (e.g. the United States). In the New Zealand context, the Parliamentary Commissioner for the Environment (PCE; 2016) reports that animal agriculture is our largest contributor of greenhouse gases. Two by-products of animal agriculture, methane and nitrous oxide, are also more potent than carbon dioxide in warming (by 25 times and almost 300 times, respectively, PCE, 2016). With our long history and focus on farming, these more potent gases make up the largest proportion of our total greenhouse gases in the atmosphere; the highest proportion out of all OECD countries (54\%; PCE).

Despite our relatively high contribution for our size, we will fare reasonably well: the UND-GAI ranks New Zealand among the most resilient countries to climate change. While we are currently ranked $17^{\text {th }}$ in terms of how exposed, sensitive, and able to adapt to the effects of climate change, this is offset by our economic, political, and social 'readiness' that ultimately places us second most resilient in the world (Chen et al., 2015; 2018).

Therefore, as some of the largest contributors to the problem are also those more likely to survive its effects, this leaves the environmental consequences to the less fortunate. As well as those in poorer nations being most vulnerable to climate change overall, disadvantaged groups within these and other countries tend to be most at risk (Althor et al., 2016), thus prompting warnings that climate change is likely to increase social inequality (IPCC, 2014b; Singer, 2018). Within NZ, and consistent with global trend, Māori (our indigenous population) have been identified as most vulnerable to climate change (Climate Change Adaptation Technical Working Group, 2017).

Local environmental problems (for instance pollution from nearby industry; O'Rourke \& Connolly, 2003) are already inequitably distributed. Climate change has the potential to intensify this inequality, and therefore is examined through an environmental 
justice framework (e.g. Agyeman et al., 2016; Schlosberg \& Collins, 2014; Jylhä, 2016). Bryant (1995) described environmental racism as occuring when people of colour are disproportionately exposed to environmental hazards, and receive less protection from these hazards, than more privileged groups. Pellow (2000) takes this idea a step further in linking environmental inequality to social hierarchy more broadly, and the burdening of low-status social groups with environmental hazards of all kinds. Indeed, individuals in subordinate groups tend to be exposed to more hazards in their jobs, and live in areas closer to landfills, pollution, and other environmental hazards (e.g. Ard, 2015; Cushing, Morello-Frosch, Wander \& Pastor, 2015; Gunn et al., 2017). Pellow argues that this occurs when competition for resources inevitibly results in dominant groups gaining disproportionate access to these resources, and low-status or minority groups having to instead face the consequences of environmental degradation.

Despite the clear injustice of this state of affairs, some individuals prefer it this way. Specifically, individuals who endorse social dominance orientation (SDO; Sidanius \& Pratto, 1999) are accepting and even prefer social inequality and hierarchical intergroup relations. They believe that high-status social groups (of which they are typically, though not exclusively, members) are at the top of the hierarchy because they are more worthy than other, subjugated groups, and that it is acceptable to enforce positions in the existing hierarchy. Research has shown the importance of SDO in predicting prejudiced attitudes and underlying support for policies relevant to intergroup relations (Asbrock, Sibley \& Duckitt, 2010; Austin \& Jackson, 2019; Ho et al., 2015; Sidanius, Cotterill, Sheehy-Skeffington, Kteily \& Cavacho, 2016). As such, they believe they deserve to disproportionately benefit from environmental exploitation and avoid environmental hazards, while disregarding the effect this has on lower-status social groups (Jackson, Bitacola, Janes \& Esses, 2013; Milfont \& Sibley, 2014).

Taken together, the extant literature suggests that SDO is an ideology that serves to reinforce environmental inequality. It is therefore important to understand its role as an ideological barrier to action on climate change and other environmental issues. Jylhä and colleagues (Jylhä, 2016, Jylhä \& Akrami, 2015; Jylhä, Cantal, Akrami \& Milfont, 2016) were the first to apply the environmental justice framework to the association between SDO and 
environmentalism. They suggest that Social Dominants ${ }^{1}$ tolerance of inequality explains why they are more accepting of the inequity in the causes and consequences of climate change. In my thesis, I contribute to this line of research to clarify the nature of the association between ideological and environmental attitudes.

\section{Overview and structure of the thesis}

In the next sections within this chapter, I review the literature to date on the predictors of environmentalism. I use the term 'environmentalism' to mean any measurable environment-relevant variable. This includes pro-environmental attitudes or behaviours, which are those that people engage in to minimize their negative impact on the environment (Kollmus \& Agyeman, 2002), and climate change belief. Others have similarly defined environmentalism broadly as "concern for the environment and support for environmentfriendly behaviours, intentions, and attitudes" (Zhao, Zhang, Xu, Lu \& He, 2018, p. 2). As such, many researchers will measure environmentalism using multiple indicators (e.g. Jia, Alisat, Soucie \& Pratt, 2015; Milfont et al., 2017; Zhao et al., 2018), reflecting its complexity.

The reason I take a broad approach to examining environmentalism is because research on environmental attitudes has both operationalised and measured these attitudes unsystematically (Milfont \& Duckitt, 2010; Stern, 1992). Researchers of environmental psychology have made use of a variety of instruments to measure environmental attitudes in what Stern (1992) has called an "anarchy of measurement" (p. 279). Furthermore, knowledge or belief in climate change does not necessarily lead to action (Kempton, Boster \& Hartley, 1995; Kollmuss \& Agyeman, 2002), therefore it is useful to cast a wider net to include associations with constructs such as pro-environmental attitudes, Green politics, and climate change anxiety, as all may yield equally informative findings.

I then elaborate on the theoretical bases of authoritarianism and social dominance, before delving into the existing literature that associates these variables with environmentalism. The literature to date is sparse but growing, with many avenues for future

\footnotetext{
${ }^{1}$ Throughout my thesis, I sometimes refer to individuals with relatively higher levels of SDO as 'Social Dominants' or High-SDOs. I do not use these terms to mean that these individuals are necessarily high in the construct with reference to the scale, but instead to mean that they are higher than most others.
} 
research. I highlight the gaps in the literature, and discuss how the program of research in my thesis aims to address these gaps.

In the chapters that follow, I describe the research that I have conducted to answer the general question, 'What is the nature of the association between ideology and environmentalism?' Studies 1A and 1B (Chapter 2) work towards this aim by synthesizing the literature to date using meta-analysis to examine the nature and strength of the relationships between SDO, RWA, and environment-relevant variables. In Chapter 3, I examine the extent to which the variables are potentially causally related by examining their longitudinal associations (Study 2A \& 2B). In Chapter 4 (Studies 3A - 3C), I review research on the two components of SDO, anti-egalitarianism and dominance, and apply this twodimensional model to predict environmentalism. In Chapter 5, I present results of thematic analyses of an interview study where students with relatively high, moderate, and low scores on the SDO scale were recruited to discuss environmental issues (Study 4). I then develop the main ideas expressed in the interviews into survey items (Study 5A \& 5B), to see how a larger group of people respond to these ideas. In my final chapter, I draw the findings from these twelve studies together to discuss their broader contribution to the literature. 


\section{Literature review}

Milfont, Milojev, Greaves and Sibley (2015) reported that just over half of New Zealanders believe climate change is happening and that humans are responsible, despite virtually unanimous agreement among climate scientists (e.g. Cook et al., 2016). Although there is a general trend of increasing public agreement, with belief in climate change and its human causes increasing over time (Milfont, Wilson \& Sibley, 2017), action on climate change tends to lag behind. Indeed, consistent with decades of research on the relationship between attitudes and behaviour (Kollmuss \& Agyeman, 2002; Rhodes \& de Bruijin, 2013), belief in climate change is only weakly related to actual engagement in pro-environmental behaviour (e.g. Hornsey, Harris, Bain \& Fielding, 2016).

In the United States, the picture is rather similar: about seventy percent believe climate change is happening, but little over half of the population agree that humans are to blame (Leiserowitz et al., 2015; 2017). Furthermore, a previous report notes that the majority (70\%) of individuals feel they need 'a lot' more information about global warming before they will be convinced (Leiserowitz, Maibach, Roser-Renouf, \& Hmielowski, 2012). Attitudes towards climate change are given special attention in my thesis owing to the evidence that climate change is the most significant environmental problem of our time (IPCC, 2014).

In early models of behaviour, it was believed that decision making follows a linear progression, where knowledge (e.g. about environmental issues) leads to an attitude about these issues (e.g. concern), prompting behaviour (Kollmuss \& Agyeman, 2002). These models implied that teaching people about environmental problems would spur action, as knowledge would increase and presumably flow through to behavioural change. This would make sense, if we take the position that the only thing stopping people from taking action is their deficit in knowledge (Burgess, Harrison \& Filius, 1998). Based on this assumption, disseminating information is sufficient to change attitudes and motivate action (Marteau, Sowden \& Armstrong, 1998). However, information campaigns do little to spur action, and therefore these simple information deficit models have been rejected (e.g. Delicado, 2012; Marteau et al., 1998; Owens \& Driffill, 2008).

Instead, awareness of the issues and potential solutions to climate change might be a necessary, but not sufficient, criterion to motivate people to choose pro-environmental behaviours. Indeed, Hines, Hungerford and Tomera (1987) showed that knowledge of the 
problem and solutions, as well as feeling ones actions would make a difference, endorsing pro-environmental attitudes, verbally committing to engage in the behaviour, and feeling personally responsible for such actions, are all important predictors of pro-environmental behaviour. However, the associations between such variables and pro-environmentalism are weak (Kollmuss \& Agyeman, 2002). In reviewing experimental work to encourage energy conservation and recycling, Abrahamse and Steg (2013) demonstrate the power of social norms for influencing behaviour. That is, people will be motivated to act pro-environmentally if they believe others close to them do so (a descriptive norm), and approve of these actions (an injunctive norm; Cialdini, 2003). Descriptive norms also emerged as one of the strongest predictors of engaging in behaviours aimed at mitigating climate change (van Valkengoed \& Steg, 2019).

These findings are consistent with other models of behaviour, such as Ajzen's (1991) theory of planned behaviour (TPB), which has been frequently applied to pro-environmental behaviour (see Gardner \& Abraham, 2008; Klöckner, 2013, for reviews of the aplication of this model). The TPB asserts that engaging in a behaviour is determined by one's behavioural intentions. Intentions are said to be reliant on an individual believing the behaviour is positive, achievable, and something that those close to them believe they should do. While intending to act does make action more likely, it does not fully explain the situation (Bamberg \& Moser, 2007).

Hornsey et al. (2016) noted that part of the difficulty in making sense of the predictors and barriers to action is that environmental research is conducted across diverse domains such as climate science, agriculture, consumer behaviour, and psychology. In an effort to improve our overall understanding of this literature, Hornsey and colleagues synthesized the varied research on the predictors of climate change denial. Using meta-analysis, they found that variables typically included in information deficit models as likely to increase our acceptance of an issue, such as knowledge about climate change and education level, showed only weak associations (i.e. $r$ 's of .18 for subjective knowledge about climate change, .25 for objective knowledge, and .12 for education in general).

Some research does show that climate change sceptics report knowing just as much about climate change as believers (e.g., Kellstedt, Zahran \& Vedlitz, 2008; Malka, Krosnick \& Langer, 2009), and that environmentalists know just as much about environmental issues as anti-environmentalists (e.g., Kempton et al., 1995). However, Hornsey et al. (2016) show 
that belief in climate change is marginally stronger with more perceived knowledge, and shows another small improvement when objective knowledge about climate change is tested. Other demographic factors, such as age and sex, were similarly minimally related. Importantly, they found that people's underlying ideologies were more important predictors of climate change belief. van Valkengoed and Steg (2019) recently confirmed that the effects of knowledge and experience of climate change on mitigation behaviours are negligible ( $r$ 's of .14 and .12), however did not include political variables in their meta-analyses.

Although they may appear at face value to be several steps removed from environmental attitudes, Hornsey et al. (2016) demonstrated that political affiliation $(r=.30)$ and ideology $(r=.15)$ were among the strongest demographic predictors of climate change denial. Those who reported voting for conservative political parties tend to deny climate change, whereas liberal party supporters accept it. This helps to explain why people's views on climate change are resistant to change, especially in the way that information deficit models propose change occurs. Instead of increased knowledge inspiring change, people's ideologies act to filter incoming information, making information about climate change consistent with their existing version of reality, rather than causing a shift in belief.

Education level works as a moderator of the association between political affiliation and climate change belief: while Republicans deny climate change and Democrats are more accepting of it, increased education only makes this divergence more extreme (McCright \& Dunlap, 2011). Going from high school to college graduates, education increases liberal's acceptance and concern about climate change, whereas conservatives become even more sceptical (Hamilton, 2011; McCright \& Dunlap, 2011). This is likely, in part, because education makes people more attuned to seeking information: Democrats and Republicans read news from different sources, which work to reinforce their existing opinions on climate change. The differences in the content of liberal and conservative sources on climate change, such as news media and political statements, demonstrate the increasing politicization of climate change.

\section{Politicization of climate change}

Climate change is an increasingly political issue, with public opinion divided over whether it represents fact or fiction (McCright \& Dunlap, 2011). The political polarization of climate change follows polarization of social issues that occurred decades earlier, such as abortion (DiMaggio, Evans \& Bryson, 1996). From the late nineties, political party 
identification served to differentiate opinions on a range of social, economic, and cultural issues, with liberal and conservative parties growing "increasingly divided on all the major policy dimensions in American politics" (Layman, Carsey \& Horowitz, 2006, p. 83). This was certainly helped along by the conservative response to the environmental movement, with conservative think tanks promoting messages aimed at undermining the legitimacy of climate change from the 1990s (McCright \& Dunlap, 2000).

McCright and Dunlap's (2000) analysis of these claims found they tended to attack the scientific basis of climate change, argue that if climate change were to happen, the consequences would be overwhelmingly positive, and maintain that mitigating action would be detrimental (e.g. to the economy, national security, or even to the environment itself). The countermovement even spotlighted the few scientists who refuted climate change, using their 'expertise' to promote their agenda (e.g. Jacques, Dunlap \& Freeman, 2008). Soon, Republican leaders in the United States were citing non-peer-reviewed research refuting climate change, while attempting to discredit peer-reviewed climate science (McCright \& Dunlap, 2003), essentially 'cherry picking' the evidence to suit their cause. This presented the debate as more uncertain than the scientific consensus, which stands at near-unanimous support (Cook et al., 2016; Doran \& Zimmerman, 2009). It is this conservative countermovement that contributed to the United States failing to ratify the Kyoto Protocol, which would have seen countries cooperating to mitigate climate change before it was too late (McCright \& Dunlap, 2003).

In the American political system, Democrats are the more liberal party (thus endorsing policies that promote government intervention and support for citizens), whilst the Republican party is comprised of conservatives (who prefer individual freedom, and limited government intervention; McCright \& Dunlap, 2011). Oreskes and Conway (2010) argue that conservatives view international agreements to reduce greenhouse gas emissions as threats to the economy and the free market, representing substantial government interference, which hence explains their lack of support for such policies. While the Kyoto Protocol was signed over twenty years ago, the more recent Paris climate change agreement is facing similar problems. Almost 200 countries signed this agreement, committing to keep global warming below two degrees Celsius above pre-industrial levels (United Nations, 2015).

With climate change still a political issue today, Republican President of the United States Donald Trump recently signalled his intention to withdraw from the Paris agreement. 
Again, despite the scientific consensus on climate change, Trump is one of the minority of people (but one of many conservatives; Stokes, Wike \& Stewart, 2015) who remains a sceptic on the issue. With the exit of the United States, one of the largest contributors of greenhouse gases (World Resources Institute, 2014), the future of the agreement is unclear, and the projected environmental and social consequences of the move to withdraw are dire (Saad, 2018).

Hornsey et al.'s (2016) meta-analysis pitted predictors of environmentalism against each other to find that political affiliation and ideology were of the strongest predictors. There has been a resurgence in interest in political ideology in the past 15 years, motivated in no small part by Jost, Glaser, Kruglanski and Sulloway’s (2003) conceptualisation of conservativism as a form of motivated social cognition. Jost and colleagues argue that holding a conservative ideology serves a suite of psychological functions, including minimizing change, reducing fear, and justifying inequality. For the purposes of this thesis, Jost et al.'s work is particularly useful in terms of how they define the components of this ideology. They argue that, historically, conservatism represents two closely aligned sets of beliefs - antiegalitarianism, and endorsement of what are perceived as legitimate authorities. These beliefs are operationalised as the ideological variables of social dominance orientation and right-wing authoritarianism. These variables are related to both political affiliation and ideology, and show potential for helping us understand people's relationship with nature, however were absent from the suite of variables analysed by Hornsey and colleagues.

\section{The conception and measurement of SDO and RWA}

Sidanius and Pratto (1999) define social dominance orientation (SDO) as the preference for social hierarchy within groups, or "the degree to which individuals desire and support group-based hierarchy and the domination of 'inferior' groups by 'superior' groups" (p. 48). The authoritarian personality values the power of perceived authority over others, with right-wing authoritarianism (RWA) comprised of three interrelated attitudinal clusters: authoritarian submission, conventionalism, and punitiveness against deviants (Altemeyer, 1981).

Both SDO and RWA relate to prejudiced attitudes. These include hostile attitudes towards people unlike oneself (the 'outgroup') and a preference for people similar to oneself (the 'ingroup', Duckitt, 2001). Owing to their position in society, which is reinforced by institutionalized discrimination, members of minority groups tend to have poorer health 
outcomes, less access to job opportunities, and are restricted to poorer quality housing (Sidanius \& Pratto, 1999). People who hold prejudiced attitudes about one out-group are also more likely to hold unfavourable attitudes towards other out-groups, demonstrating the generality of prejudice (Duckitt, 2001; Everett, Caviola, Savulescu \& Faber, 2018; Hadarics \& Kende, 2018). Across various samples and targets of prejudice, the association between prejudiced attitudes towards out-groups is strong (e.g. Adorno, Frenkel-Brunswick, Levinson $\&$ Sanford, 1950; Altemeyer, 1981; 1996). Therefore, disliking one group of people is strongly predictive of disliking other groups, prompting research on what underlies this phenomenon.

Adorno et al. (1950) sought to explain generalized prejudice by theorizing the authoritarian personality. They argued that favouritism toward the in-group, and prejudiced attitudes towards the out-group, conservative political and economic views, as well as profascist attitudes, combined to form the authoritarian personality, dubbed an 'attitudinal syndrome'. Their work initially sparked a great deal of interest and research into the authoritarian personality, especially in relation to prejudice (e.g., Campbell \& McCandless, 1951; Hites \& Kellogg, 1964; Martin \& Westie, 1959), however attention turned away from authoritarianism in the 1960s. Altemeyer (1981) revived interest in this area with the development of an improved measurement instrument, the right-wing authoritarianism scale (RWA scale). Altemeyer's RWA scale measured just three of Adorno et al.'s original nine components of authoritarianism, as he believed that only conventionalism, authoritarian aggression, and authoritarian submission covaried to the extent needed to form a reliable scale.

Altemeyer (1981) defined conventionalism as "a high degree of adherence to the social conventions which are perceived to be endorsed by society and its established authorities" (p. 148). This means that right-wing authoritarians support traditional social norms, often guided by religion, and believe these traditional norms ought to be followed. Authoritarian aggression is the endorsement of harm against those who transgress from these norms. This is expressed through prejudice, or even outright violence, which individuals high in RWA perceive to be approved of by those in leadership roles to whom they submit to. This authoritarian submission is the tendency to follow, trust and support those in legitimate authority roles, and to be hostile to those who do not. Altemeyer argues that true right-wing authoritarians endorse all three clusters of attitudes, a position that has not gone without challenge. Altemeyer (1981) showed RWA to relate to aggression in a Milgram-type study, 
acceptance of transgressions by government officials, and punitiveness towards ordinary citizens who defied the law.

The role of hierarchy and anti-egalitarianism has become a particular focus of research on group conflict over the past two decades, with the articulation of social dominance theory (SDT) in the early nineties (Pratto, Sidanius, Stallworth \& Malle, 1994; Sidanius \& Pratto, 1993). Sidanius and Pratto noted that humans are organised in systems of group-based social hierarchies. Within these, the dominant groups enjoy more positive social value (e.g. better jobs, health care, political authority and power), whereas subordinate groups are disproportionately allocated negative social value (e.g. high-risk jobs, poor healthcare, harsher punishment for breaking the law). These groups are based on an age system (where adults are dominant to children), gender system (patriarchy, where men are dominant over women), and arbitrary-set systems. In arbitrary-set systems, group formation depends on situational factors, such as race, social class, or religion. One basic assumption of SDT is that all social systems have age and gender hierarchies, however arbitrary-set systems only emerge in societies with economic surplus, and these systems tend to be associated with the most violence and oppression. When there is an abundance of resources, people no longer need to devote their time to survival (e.g. hunting or gathering crops), thus making way for new specialized roles, such as in the military, which work to maintain hierarchy based on arbitrary characteristics. It is because of these group-based hierarchies that social systems experience conflict.

Sidanius and Pratto (1999) further explain that societies function to minimize this intergroup conflict by endorsing legitimizing myths that support inequality and discrimination. These legitimizing myths are "attitudes, values, beliefs, stereotypes, and ideologies that provide moral and intellectual justification for the social practices that distribute social value within the social system" (p. 45). The myths justify social inequality (hierarchy-enforcing), but may also be opposed by countervailing accounts that promote social equality (hierarchy-attenuating). For example, meritocracy is the hierarchy-enforcing belief that people at the top of the hierarchy are there because they earnt their place (and those at the bottom similarly deserve to be there). Hierarchy-attenuating beliefs include feminist and socialist arguments, which portray the idea that everyone ought to have the same human rights and society should be equal. 
Social dominance orientation (SDO) is the measurable ideological construct, at the centre of tests of SDT, that epitomises endorsement of intergroup inequality and social hierarchy. People scoring higher in SDO tend to gravitate towards hierarchy-enhancing jobs, such as police officers, compared to hierarchy-attenuating roles (e.g. charity worker). They oppose policies that aim to redistribute social value. Even when individuals are assigned to a group based on arbitrary grounds, Social Dominants allocate their own (meaninglessly selected) group more social value than the other group (Sidanius, Pratto \& Mitchell, 1994). Although members of the dominant group tend to endorse these ideas to a greater extent, subordinates are also complicit in supporting ideas that maintain their place at the bottom of the hierarchy (Lee, Pratto \& Johnson, 2011; Sidanius \& Pratto, 1999).

Sidanius and Pratto (1993) created the SDO scale to measure this construct, which includes items such as "Some groups of people are simply inferior to other groups" and "All groups should be given an equal chance in life" (con-trait). For a discussion on recent advancements in the measurement of SDO, see Chapter 4. SDO predicts many of the same social, political, and ideological variables, as well as prejudice and ethnocentrism (e.g. Jost \& Thompson, 2000; Pratto et al., 1994; Whitley, 1999) as RWA. Given the similarities in their predictive ability, one might expect that the RWA and SDO scales measure the same underlying construct. However, SDO and RWA are typically only weakly related, if at all (e.g. correlations typically range from $r=.07$ to .28; Duckitt, 2001). While together they predict about $50 \%$ of the variance in generalized prejudice, each variable explains unique variance, predicting prejudice independent of each other (e.g. Altemeyer, 1998). Altemeyer (2003), having once believed High-RWAs were the "the most prejudiced group of people the social sciences had ever found" (p. 162), reported later changing his mind, and instead acknowledged endorsers of SDO as even more prejudicial.

Altemeyer's (1998) research outlined various differences between SDO and RWA. He found that those who strongly endorse RWA tend to be religious, though high scorers on SDO are not especially so. Social dominants usually know they are more prejudiced than average, whilst Authoritarians do not. Men typically score higher on SDO than women, whereas scores on the RWA scale are roughly equivalent for both men and women. Altemeyer described Social Dominants as 'leaders', who want to ensure their ingroup, who they believe are superior to other social groups, are in more powerful positions. In contrast, the Authoritarian prefers to be a 'follower': they are careful to follow social norms and enforce them, supporting policies and actions that punish those that digress. 
Despite these differences, Adorno et al. (1950) would argue that RWA and SDO are two sides of the same coin: both part of the authoritarian personality, with SDO as the dominant side, and RWA as submissive. Duckitt (2001) presents a thoughtful discussion of the theoretical origins of SDO and RWA. He predicts that an individual's early socialization leads to repeated activation of a particular schema, which encourages certain personality traits, in turn influencing the way they see the world. This worldview in turn motivates individuals to favour SDO or RWA-consistent ideologies. As RWA is associated with social threat (e.g., Altemeyer, 1998), it likely arises from socialization that supports a threat schema: parenting that is harsh and punitive. This prompts the individual to conform (whereas tolerant parenting fosters autonomous personality traits). Because the individual supports conformity, they resist change or disruption to social order. This leads them to see the world as threatening and dangerous, thus making the motivational goal of social control and security more salient, characterised by support for authoritarian and conservative ideological beliefs.

Duckitt (2001) goes on to argue that portrayals of the world as a competitive jungle, where one has to be ruthless to survive, are associated with SDO, and not RWA. He posits that unaffectionate parenting fosters a toughminded, ruthless personality, which leads to this competitive worldview. This ultimately results in an individual who endorses SDO through the motivational goal for dominance and superiority. On the other hand, for those scoring low in SDO, parenting was likely affectionate, promoting compassion and empathy, and a view of the world as cooperative. These individuals would be motivated to show concern for others and be altruistic, leading them to endorse egalitarianism. Extensive research has now validated the dual-process model for its utility in predicting intergroup attitudes (Duckitt \& Sibley, 2010; Duckitt, Wagner, du Plessis \& Birum, 2002).

Unsurprisingly, measurement of SDO and RWA therefore have an inherent focus on social relations. For example, the SDO scale centres on attitudes towards social groups and their positioning, for example "some groups of people are simply inferior to other groups" (Ho et al., 2012). Items constructed to measure RWA similarly align with social relationships, i.e. deferring to authority (people or groups in higher power positions) as in submission/conservatism, ("What our country really needs is a strong, determined leader who will crush evil, and take us back to our true path"). Despite this, both variables relate to human-nature relations in that those endorsing SDO and RWA tend to express lower proenvironmental attitudes (Jackson et al., 2013; Schultz \& Stone, 1994), scepticism about climate change (Hoffarth \& Hodson, 2016; Milfont, Richter, Sibley, Wilson \& Fischer, 
2013), and an unwillingness to act on the problem. In the next section, I review the research to date that relates SDO and RWA to environmentalism.

\section{The relationship between SDO, RWA and environmentalism}

Perhaps the first research into the ideological predictors of environmentalism came from Peterson, Doty and Winter (1993), who broadened the application of RWA to examine its association with contemporary social attitudes. While an abundance of research had demonstrated the utility of RWA in predicting intergroup attitudes, the researchers correlated RWA with attitudes on AIDS, drug use, and environmental issues. They show that RWA is implicated in prejudice towards both those with AIDS and drug users, likely owing to their status as 'out-groups' to which authoritarian aggression is directed, and the threat AIDS and drugs pose to the 'American way of life'. Across three studies, Peterson et al. (1993) demonstrated that while RWA did not relate to statements about punishing polluters, high scores on RWA were associated with beliefs that environmentalists should be punished. Authoritarians also tended to agree that action on environmental issues is likely to reduce America to a 'second-rate power', and that environmental issues are exaggerated or blown out of proportion. Therefore, in the context of environmental issues, authoritarian aggression is directed towards those working to mitigate the problem, as their actions are threatening to the existing way of life, rather than those potentially causing the problem.

Peterson et al.'s (1993) work spurred new interest on the application of Authoritarian theory to environmental issues. Schultz and Stone (1994) followed, reporting that greater endorsement of RWA is associated with less concern about the environment, and the rejection of pro-environmental attitudes. Noting the apparent concern that environmental action is detrimental to a country's growth (as demonstrated by Peterson et al.'s work), Schultz and Stone proposed that Authoritarians' preference for economic growth underlies their rejection of pro-environmental attitudes. Both Thibodeau (1998) and Wang (1999) subsequently replicated the negative relationship between RWA and pro-environmental attitudes.

Pratto et al. (1994) presented the first investigation of the association between SDO and environmentalism. They showed that Social Dominants were less supportive of policies aimed at protecting the environment, which they attributed to Social Dominants opposition to any policy that promotes equality between humans and nature (which includes non-human species). Wang (1999) also showed females' scores on SDO predicted environmental 
attitudes, with Social Dominants less likely to endorse pro-environmental positions. Because Wang shows pro-environmental attitudes to relate to attitudes towards women, he argues that human dominance over nature parallels male dominance over females, a position called ecofeminism (Warren, 1990). Milfont et al. (2013) echoed this sentiment by similarly explaining the relationship between SDO and environmentalism as owing to endorsement of SDO promoting "human hierarchical dominance over nature" (p. 1127). These explanations are consistent with the dominant social paradigm (DSP; Noe \& Snow, 1990). Feygina (2013) explains that those supportive of the DSP position themselves to dominate over nature and exploit the environment.

Since these earlier studies, a growing number of recent studies have highlighted the association between SDO and environmentalism. Those who endorse SDO tend to deny climate change and its human causes (e.g. Jylhä \& Akrami, 2015; Jylhä et al., 2016; Häkkinen \& Akrami, 2014; Milfont et al., 2013, Study 4), are less concerned about the environment (Milfont et al., 2013, Study 1), and more accepting of the use of natural resources (Milfont \& Duckitt, 2010; Milfont et al., 2013, Study 3). Additionally, countries with relatively higher mean levels of SDO tend to have weaker environmental policies (Milfont et al., 2013, Study 2). Both SDO and RWA are related to weaker support for Green political parties (Sibley \& Wilson, 2007; Van Hiel, Cornelis, Roets \& Clercq, 2007). Furthermore, people who endorse these ideologies tend not to believe there are benefits from acting pro-environmentally (Van Heil \& Kossowska, 2007), or that we should preserve nature (Milfont et al., 2013). Authoritarians are more likely to agree that acting on environmental issues will be costly for their country (Caddick, 2016; Peterson et al., 1993) and believe that environmentalists (and not polluters) are the problem (Peterson et al., 1993). Authoritarians and Social Dominants seek to deflect responsibility for taking action on global warming (Vail \& Motyl, 2010).

A more recent viewpoint on what underlies the SDO-environmentalism link is that Social Dominants support exploitation of natural resources as a means to gain dominance over other social groups. This is the argument made by Milfont and Sibley (2014) in their hierarchy enforcement hypothesis of environmental exploitation. By this view, those endorsing SDO support environmental exploitation when doing so will increase the gap between high- and low-status groups. Finding support for this hypothesis, they show that Social Dominants only support the building of an environmentally hazardous mine when the social elite are set to gain. Jackson et al. (2013; Study 2) similarly demonstrated that an 
individual's level of SDO relates to support for a mine only when their ingroup will benefit by gaining disproportionate access to the resources it produces. High-SDOs are even content directing environmental hazards to poorer areas when their group will benefit (Study 3). Therefore, reminiscent of Pellow's (2000) environmental inequality theory, SDO might motivate environmental exploitation when this serves to maintain the social hierarchy, rather than the desire for dominance over the natural world itself.

\section{Summary of research gaps and future directions}

In reviewing the literature to date on the association between SDO and environmentalism, some clear gaps in the literature emerge. In this section, I highlight these gaps and explain how the collection of studies in my thesis aim to address these and therefore contribute to the wider literature on the ideological predictors of environmentalism.

Lack of cohesion in the literature. While the research on the association between SDO and environmentalism is small, it is growing. Despite this, recent efforts to synthesize the literature on the correlates of climate change belief and action failed to include SDO (Hornsey et al., 2016; van Valkengoed \& Steg, 2019). Research also demonstrates that the related ideological variable, RWA, may be relevant in understanding people's relationship with nature. While both SDO and RWA separately relate to environmentalism (e.g. Pratto et al., 1994; Peterson et al., 1993), when studied together, research tends to indicate that SDO is a stronger predictor (Milfont et al., 2013), and the association with RWA is sometimes not significant once the effects of SDO are controlled for (Häkkinen \& Akrami, 2014). Work is needed to synthesize the research to date on the ideological predictors of climate change, while pitting these predictors against each other to see which more strongly relates to environmentalism, and under what conditions. This is my aim in Chapter 2 (Studies 1A \& 1B).

In Study 1A, I synthesize the research to date using meta-analysis to examine which variable has the strongest overall relationship with environment-relevant variables. In testing the conditions which foster these associations, I consider the influence of moderating variables such as how the concepts are measured and whether participants were sampled from universities or the general population. In Study 1B, I use regression analysis to statistically control for each ideological variable and meta-analyse only the unique associations between SDO and environmentalism, and RWA and environmentalism, as a more thorough (though less complete) examination of which ideology more strongly relates to environmentalism. 
The need for longitudinal research. There are two competing predictions as to why the relationship between SDO and environmentalism exists. The first argument is that SDO relates to environmentalism through Social Dominant's desire for dominance over nature (Pratto et al., 1994; Wang, 1999, Milfont et al., 2014). A second, and more empirically supported thesis (e.g. Jackson et al., 2013; Milfont \& Sibley, 2014) is that Social Dominants want access to natural resources so that they can allocate high-status, dominant groups a greater share than subordinate, low-status groups, hence using nature as a way of enhancing the social hierarchy.

In making conclusions about why the relationship between these variables exists, researchers imply a causal model where endorsement of SDO influences later environmental attitudes. However, to my knowledge all research to date is cross-sectional, and without longitudinal data it is not possible to stipulate the direction of the association between ideology and environmental attitudes. Furthermore, these analyses cannot determine which ideology (SDO or RWA) more strongly predicts decreases in climate change belief and proenvironmental attitudes over time. In Chapter 3, I examine the longitudinal associations between these variables, firstly using a student sample (Study 2A), and then in a large-scale national probability sample (Study 2B).

Which aspect of SDO relates to environmentalism? Ho et al. $(2012,2015)$ recently made the case that SDO is comprised of two dimensions: SDO-Dominance (SDO-D) and SDO-Egalitarianism (SDO-E). They show that the two aspects of SDO differentially predict intergroup attitudes: specifically, SDO-D relates to overt racism, while SDO-E is characterized by subtle support for inequality. Despite this, no research to date has used the two dimensional model of SDO to examine how SDO-D and SDO-E relate to environmentrelevant variables.

This is an interesting research question, given the competing perspectives for the association between SDO and environmentalism. The argument of human dominance over nature is in line with an SDO-D explanation, whilst the argument that the relationship exists to maintain the hierarchy by way of environmental exploitation supports an SDO-E interpretation. In Chapter 4, I apply the two-dimensional approach of SDO to environmentalism, examining how SDO-D and SDO-E relate to environment-relevant variables (Studies 3A, 3B \& 3C). 
Qualitative work. Another limitation in the existing research is that all research to date relies on quantitative methods to examine Social Dominants views on the environment. While this work does demonstrate that a statistical relationship exists between the variables using survey-based data, no research to date has adopted qualitative methods to probe the nature of this association. In Chapter 5, I present findings of the first interview study examining self-reported reasons that those endorsing high, moderate, and low levels of SDO cite for their opinions on environmental issues. Study 4 presents a thematic analysis of interview transcripts to identify the key ideas offered by participants. In some exploratory analyses, I also examine whether and how SDO relates to these ideas. I follow this up in Chapter 6 with a pair of studies examining how the main ideas from the interview study apply to a larger group of people, and how they relate to scores on the SDO scale, using quantitative analyses (Study 5A \& 5B).

\section{Conclusion}

In sum, the literature to date demonstrates both the importance and challenges of proenvironmentalism and acting on climate change, with ideology one possible barrier to action. The program of research in my thesis works to clarify the nature of the association between ideological and environmental attitudes. In the General Discussion, I look at the key findings and contributions as a whole, and discuss what these mean for research in this area, how they may be applied, and the next steps. 


\section{Chapter 2}

\section{Ideology and environmentalism: A meta-analysis ${ }^{2}$}

\section{Introduction to Study $1 A$ and $1 B$}

Given the pressing nature of climate change, and the rapid developments in research on climate-related belief and behaviour, it is important to systematically review research informing our understanding of pro- and anti-environmentalism. Responding to this task, Hornsey et al. (2016) recently reported meta-analyses of the rapidly growing body of research on the correlates of climate change belief. Metaanalysis refers to a suite of techniques that synthesize results across multiple studies, effectively combining the statistical power of all participants included in each sample and comparing effect sizes based on differences within studies (Harrison, 2011).

Among the variables they identified as relating to belief in climate change, political ideology and affiliation showed stronger associations than demographic variables, and even surpassed the predictive ability of intuitively related variables, such as knowledge about climate change. Summarizing the link reported in 20 papers, people who vote for more conservative or right-wing political parties are more likely to deny climate change, whilst liberal political party voters are more likely to accept it. This suggests that how someone votes, and the ideological basis upon which they vote, is more important for understanding their beliefs about climate change than their degree of knowledge about the issue.

As I explained in Chapter 1, whilst Hornsey et al.'s (2016) research generated valuable information, the authors did not include SDO and RWA as correlates of climate change belief. Both SDO and RWA are intrinsically linked to political

\footnotetext{
${ }^{2}$ Note. Study $1 \mathrm{~A}$ and $1 \mathrm{~B}$ have been rewritten for inclusion in the thesis from the publication:
}

Stanley, S. K., \& Wilson, M. S. (2019). Meta-analysing the association between social dominance orientation, authoritarianism, and attitudes on the environment and climate change. Journal of Environmental Psychology, 61, 46-56. 
affiliation (Wilson \& Sibley, 2013) and political ideology (Jost et al., 2003), while also relating to environmentalism (e.g., Häkkinen \& Akrami, 2014; Milfont et al., 2013). This omission reflects Hornsey et al.'s narrow focus on studies reporting associations with climate change belief, one aspect of environmentalism, and one that had been the focus of very few studies incorporating SDO and RWA.

However, research shows that ideology relates to a broader range of beliefs, attitudes, and actions on environmental issues, than the specific question of endorsement or opposition to the reality of climate the change (e.g. Hoffarth \& Hodson, 2016; Milfont et al., 2013). The authors also completed their literature search in early 2014, thus missing the wealth of recent studies examining the link between ideological variables and environmentalism. This growing pool of literature has more than hinted at the importance of SDO and RWA for our understanding of ideology as a barrier to belief and action on environmental issues (e.g., Milfont et al., 2013). My aim is to address this gap in the literature by conducting meta-analyses of the association between environmentalism (broadly defined) and SDO and RWA.

SDO and RWA are related to a range of anti-environmental sentiments. For instance, people who score higher in SDO and RWA tend to be less convinced that climate change is happening or that humans contribute to the problem (Jylhä \& Akrami, 2015; Jylhä et al., 2016; Häkkinen \& Akrami, 2014; Milfont et al., 2013, Study 4). However SDO and RWA is also associated with lower concern about the environment (Milfont et al., 2013). support for exploitation of natural resources (Milfont \& Duckitt, 2010), and opposition to Green politics (Sibley \& Wilson, 2007; Van Hiel et al., 2007).

Taken together, previous literature (summarized in Chapter 1) supports the notion that climate change belief is not the only environmental correlate of SDO and RWA, but also that ideologies work to predict other important aspects of environmentalism. However, they might not do so to the same extent. Using regression analyses, researchers are better able to pinpoint the portion of the variance each ideological variable explains in environmentalism. When both SDO and RWA are included as predictors in regression analyses, RWA tends to yield a weaker association with environmentalism (e.g. Häkkinen \& Akrami, 2014; Milfont et al., 2013), perhaps indicating that hierarchical attitudes more strongly underpin 
environmental attitudes than do Authoritarian attitudes. However, some research reveals that the ideologies exhibit correlations with environmentalism of comparable sizes (e.g., Devine-Wright, Price \& Leviston, 2015), with some even showing RWA to be the stronger correlate (e.g. Sibley \& Wilson, 2007).

In this chapter, I present the results of two sets of meta-analyses. In Study 1A, meta-analyse the correlation coefficients from studies that examine the relationship between environment-relevant variables and SDO and/or RWA. Study 1B expands on this by examining only the unique association each ideological variable has with environmentalism. 


\section{Study 1A}

\section{Social dominance orientation and authoritarianism: Meta-analyzing the association between ideology and environmentalism}

My aim in Study 1A was to estimate the meta-analytic association between each ideology of interest (SDO and RWA) and environmentalism. In two main metaanalyses, I collected correlations between environmentalism and SDO and RWA to estimate the overall association between these variables. I also conducted a series of separate, smaller meta-analyses to analyse the associations at different levels or indicators of environmentalism. On the balance of findings from previous studies discussed above, I expected SDO would be more strongly related to environmentalism overall than RWA. However, I also considered the effect of several potential moderating variables to help explain the inconsistency in the literature.

These potential moderators were scale type (the measure used to assess SDO and RWA) and population type (whether samples were recruited from student or general population samples). Furthermore, to examine the influence of gender, the percentage of females in each sample was included in a meta-regression.

The first moderator, scale type, was included because there is some evidence that the variation in the strength of the association exists because of the way the variables are measured. For instance, some research revealing a weak or null association between RWA and environmentalism measures RWA using a subset of items or an alternative measure (e.g., Flouri, 2004; Milfont et al., 2013), and those that employ the full scale do tend to demonstrate that the variables are related (i.e. Peterson et al., 1993). Therefore, perhaps the inconsistency in findings arises at least in part from the way RWA is measured, with studies relying on shortened scales undermining the 'true' association by drawing from items that are perhaps less strongly associated with human-environment relations.

The same problem might occur when SDO is measured using shortened scales. Häkkinen and Akrami (2014) conducted two studies, showing firstly a moderate association between SDO and environmentalism using a full scale measure, 
but in the second study the association was weak; one difference being that they changed to a shortened version of the same scale. Research to date is yet to systematically explore how well these ideological variables, measured in various forms, predict environmentalism.

Gender may also be relevant to the ideology-environmentalism link. There is a well-documented increase in climate change denial among white males who are politically conservative (McCright \& Dunlap, 2011; Milfont et al., 2015; Milfont \& Sibley, 2016). Jylhä et al. (2016) noted that SDO mediates the link between political orientation, gender, and denial of climate change. Therefore, they explain that this "conservative male" effect exists because men and those who express a conservative political affiliation tend to score higher in SDO. Pratto, Stallworth and Sidanius (1997) similarly demonstrated that sex is linked to political attitudes through SDO. Based on these analyses, I expect that the association between SDO and environmentalism is stronger in studies with a greater proportion of male participants than female.

In terms of RWA, Altemeyer (1996) noted only one study that showed a gender difference in mean RWA scores. He otherwise stated that men and women have always yielded equivalent levels of RWA. This exception showed that mean levels of RWA are higher for men than for women. Since then, however, Brandt and Henry (2012) collected data from over 50 societies to show that authoritarian values are endorsed by women to a greater extent than by men, which they argued speaks to the psychological threat posed by being a member of a stigmatized group. However, Brandt and Henry measure authoritarianism as preference for authoritarian childrearing practices, which is not necessarily equivalent to endorsement of RWA. No research to date has explicitly explored the possibility of gender differences in the RWA-environmentalism association. I include gender as a continuous moderator of this relationship, however the goal of this analysis is exploratory and I make no specific prediction for the results.

In sum, I expect that both SDO and RWA will have significant, moderate associations with environment-relevant variables $(\mathrm{H} 1)$, though the mean correlation will likely be stronger with SDO than RWA (H2). I also hypothesize an effect of scale type, with full scale measures yielding stronger relationships than shortened scales or 
alternative measures (H3). Due to the restricted range of responses obtained from student samples, I expect general population samples to exhibit stronger associations between these variables (H4). Furthermore, I expect the relationship between SDO and environmentalism to be moderated by gender, with a stronger association found when there is a greater proportion of males in the sample (H5), and make no specific prediction about the moderating influence of gender on the RWA-environmentalism relationship.

I take a broad approach by including many environment-relevant variables to meta-analyze the associations these have with ideology. It is yet to be fully investigated whether the aspect of environmentalism that a certain measure taps into has an influence on the strength of the relationship with ideology. However, there are several reasons to expect that some measures are more strongly related than others.

Perhaps the most direct evidence of how the framing of environmental issues affects the strength of the relationship comes from Peterson et al. (1993). Peterson and colleagues demonstrated that the degree to which Authoritarians agree with statements about environment-related issues depends on the wording: RWA is unrelated to the belief that polluters should get more than just fines, but strongly associated with statements that environmentalists should be punished. Their series of correlations show that Authoritarians are more opposed to the environmental movement, and more accepting of polluters, than those who score low on RWA, but the associations vary depending on what aspect of environmentalism is highlighted. Therefore, the way in which researchers conceptualize environmentalism might affect how strongly it relates to ideological variables, thus demonstrating the need for separate meta-analyses to examine how SDO and RWA relate to different aspects of environmentalism.

Some research shows that SDO and RWA differentially relate to aspects of environmentalism. For example, in terms of valuing the environment, Dimdins, Sandgren and Montgomery (2016) found SDO to relate more strongly than RWA. This is a similar pattern of results as obtained by Häkkinen and Akrami (2014), who indexed environmentalism using a measure of climate change denial. I therefore expect that these measures, which broadly fit the categories of pro-environmental attitudes and climate change belief, will be more strongly associated with SDO than 
with RWA, as well as similar constructs such as support for action on climate change and climate change anxiety (H8).

In terms of support for the Green party, on the other hand, Sibley and Wilson (2007) show RWA to be more strongly related, at least among New Zealanders. When thinking about the potential reasons behind the associations, it might make sense for Authoritarians to oppose Green politics, as this represents a substantial threat to tradition and established authority. Therefore, I expect the mean correlation to be stronger between RWA and Green politics than for SDO (H9).

Another aspect of environmentalism is environmentalist threat, encompassing negative attitudes or fear towards environmentalists or action on climate change. Milfont et al. (2015) showed that SDO, and not RWA, predicts prejudice towards derogated groups: a measure that includes environmentalists. They explain this association as owing to the fact that those high in SDO disapprove of derogated groups' subordinate status. However, environmentalists might also be perceived as belonging to a threatening group and therefore disliked by Authoritarians. Indeed, Hoffarth and Hodson (2016) show SDO and RWA have comparable correlations with viewing environmentalists as threatening to the economy and the current way of life. I therefore expect SDO and RWA to have comparable associations with environmentalist threat (H10), even though the relationships might exist for different reasons.

Taken together, there is evidence to suggest that the strength of the association will depend on how environmentalism is measured. However, it is also possible that the range of correlations is what would be expected by chance, and that all are roughly equivalent measures of a general construct of 'environmentalism'. This is why, to test for these possibilities, I conduct separate meta-analyses for each type of environmental measure to assess the overall relationships of each with SDO and RWA, as equivalent associations for each environmental index would suggest environmentalism is a single, general construct. 


\section{Method}

\section{Data collection}

Sources were predominantly drawn from databases of published literature and theses. I also consulted the reference lists of papers I include, as well as studies citing these papers, for additional records. Finally, I endeavored to email researchers who have published or conducted theses in the area for unpublished research, to avoid publication bias influencing the results. I excluded studies reporting data already published elsewhere, so that information from the same sample was used only once. Furthermore, I excluded studies where an alternative measure of RWA was employed ${ }^{3}$.

One important consideration for meta-analyses is to ensure that samples are independent, so that data from the same sample are not included more than once. I faced this problem because many of the studies that I identified included more than one measure of environmentalism, meaning that multiple correlations were available from each study, all representing data from the same participants. There are several solutions to this problem, such as choosing to include only one outcome measure per study, treating each correlation as independent and including the correlations for all variables, or including the mean of the correlations for each sample (Scammacca, Roberts \& Stuebing, 2014).

The first solution raises problems over deciding which measure to include. If, as we predict, some environmental variables are more strongly related to ideology than others, then we may inadvertently over- or under-estimate the mean correlation by choosing to include certain variables over others. Choosing a variable at random

${ }^{3}$ I excluded proxy measures of RWA because I found that a significant portion of the variance in the RWA-environmentalism association was attributable to scale type $(\mathrm{Q}(4)=$ $26.23, \mathrm{p}<.001)$, with comparable associations between full scale $(-.264,95 \%$ CI [-.319, $.208])$ and shortened measures $(-.223,95 \%$ CI $[-.292,-.151])$ of the RWA scale, as well as the Zakrisson measure (-.322[-.445, -.187]) and the ACT scale (-.282, [-.371, -.187]). However, our 'other' category, which included alternative measures of authoritarianism such as cultural or social conservatism (as in Ray \& Wilson, 1975; Van Heil \& Kossowska, 2007), preference for authoritarian child-rearing practices, and support for authority (e.g. Flouri, 2004), had no relation with environmentalism $(\mathrm{r}=-.063[-.132, .007], \mathrm{p}=.079)$. The meta-analytic correlation was significantly different to that when measured by all other measures of RWA (as evidenced by the nonoverlapping confidence intervals). I therefore decided to exclude studies that used these types of measures, as they may not be tapping the same construct, and their results vary widely. The PRISMA diagram has been updated to reflect that these studies have been excluded. 
does somewhat mitigate the potential bias, but some information is lost using this method. The second solution, which ignores dependence of effect sizes, inflates the variance of the mean correlation and gives more weight to studies with more effect sizes, thus introducing bias (Scammacca et al., 2014). Including effect sizes as though they contribute independent information assumes there is no correlation between measures (Borenstein, Hedges \& Higgins, 2009), which is inappropriate when each variable is a related but distinct measure of environmentalism. The third solution, including the average of the correlations from each sample, is perhaps the most common approach (Ahn, Ames \& Myers, 2012). Despite this, Borenstein et al. (2009) notes that including an average effect size per study assumes all measures are perfectly correlated, all measuring the same construct, and thus able to be directly combined. However, this method is fair in that it only includes one effect size per independent sample, and does not lose any information by excluding certain measures (instead, it averages them all, thus possibly converging on a more accurate correlation between two variables).

I conducted each of the main meta-analyses three times to conform to each of the solutions to non-independence discussed above. Notably, all effect sizes were very similar regardless of the method employed, however the variance did change substantially. Specifically, including one variable per study (chosen at random) yielded the strongest effect sizes for both SDO and RWA, suggesting that these results may warrant caution as this method is perhaps overestimating the mean effect size. Treating each correlation as independent roughly quadrupled the Q statistic, a measure of heterogeneity of the included studies, and thus has the effect of inflating the variance as expected (Scammacca et al., 2014). Using the average correlation from each unique sample obtained the lowest mean effect size for SDO, and an estimate for RWA that fell in between those obtained by the other two methods. Measures of heterogeneity were also lower using this approach, suggesting that on balance this is a good solution.

I decided to combine the best of these solutions to firstly yield an overall estimate by including the average of all relevant correlations for each sample. I used this method for the main meta-analyses, and used this model to test my proposed moderating variables. I then followed Cooper's (1998) recommendations, averaging similar outcome types and including these in separate meta-analyses for six categories 
of environmental variables: belief in climate change, support for action, environmentalist threat, green politics, pro-environmental attitudes, and climate change anxiety. Although running separate meta-analyses limits the researcher from making inferences about the overall effect of all studies (as the full corpus of studies are not included in every meta-analysis), I avoid this limitation by also conducting the full meta-analyses that includes the average correlation from all studies. This method is valuable as it allowed me to retain the associations from each study to test our specific hypotheses about the relation between ideology and different facets of environmentalism. This ensures that information is not lost from the individual studies, whilst also maintaining statistical independence (Scammacca et al., 2014).

\section{Data extraction}

As my interest lies in the relationship between SDO, RWA, and environmentrelevant variables, I looked for papers that assessed at least one environmental variable and either SDO or RWA. My main search terms is included below, attempting to capture all research items that might include either SDO and RWA, and at least one aspect of environmentalism:

((RWA OR Authoritarianism OR “right-wing authoritarianism”) OR (SDO OR “social dominance" OR "social dominance orientation")) AND (“climate change" OR environmentalism OR “global warming” OR "green party" OR proenvironmental OR "green politic*”)

I used this search term to consult PsycINFO, a psychology-specific search engine, as well as Scopus and Web of Science. When searching for theses, the terms appeared to be too broad, as they were capturing too many items unrelated to our interests (e.g. RWA can also mean "random walking algorithm" or "Russian wheat aphids"). Therefore, when searching ProQuest Dissertations and Theses Global, I narrowed my search term to: ("right-wing authoritarianism" OR "social dominance orientation") AND (“climate change" OR environmentalism OR "global warming” OR "green party" OR proenvironmental OR "green politic*”).

In case these searches missed any records, I also searched Google Scholar using my search terms, and again using key terms within the 'cited by' lists for all articles we included in the meta-analyses. Furthermore, I searched ResearchArchive, a 
New Zealand thesis database. I was also able to include fourteen of my supervisors own unpublished datasets, plus two from a colleague.

Where possible, I took correlations from published papers or theses. Where the correlation(s) were not available within the paper, I emailed the corresponding author to request this, and worked my way through the list of authors if I received no response. In ten cases, I was unsuccessful in my attempts to obtain this information from the researchers, either because they could not be contacted, or because they no longer had the data required to compute the correlations. I also contacted all authors (that I could find contact details for) who had published or conducted theses in this area to enquire about unpublished work. The PRISMA diagram (Figure 2.1) details how the final sample of 44 studies (in total) were identified. Appendix A has a full list of all studies included in these meta-analyses. 


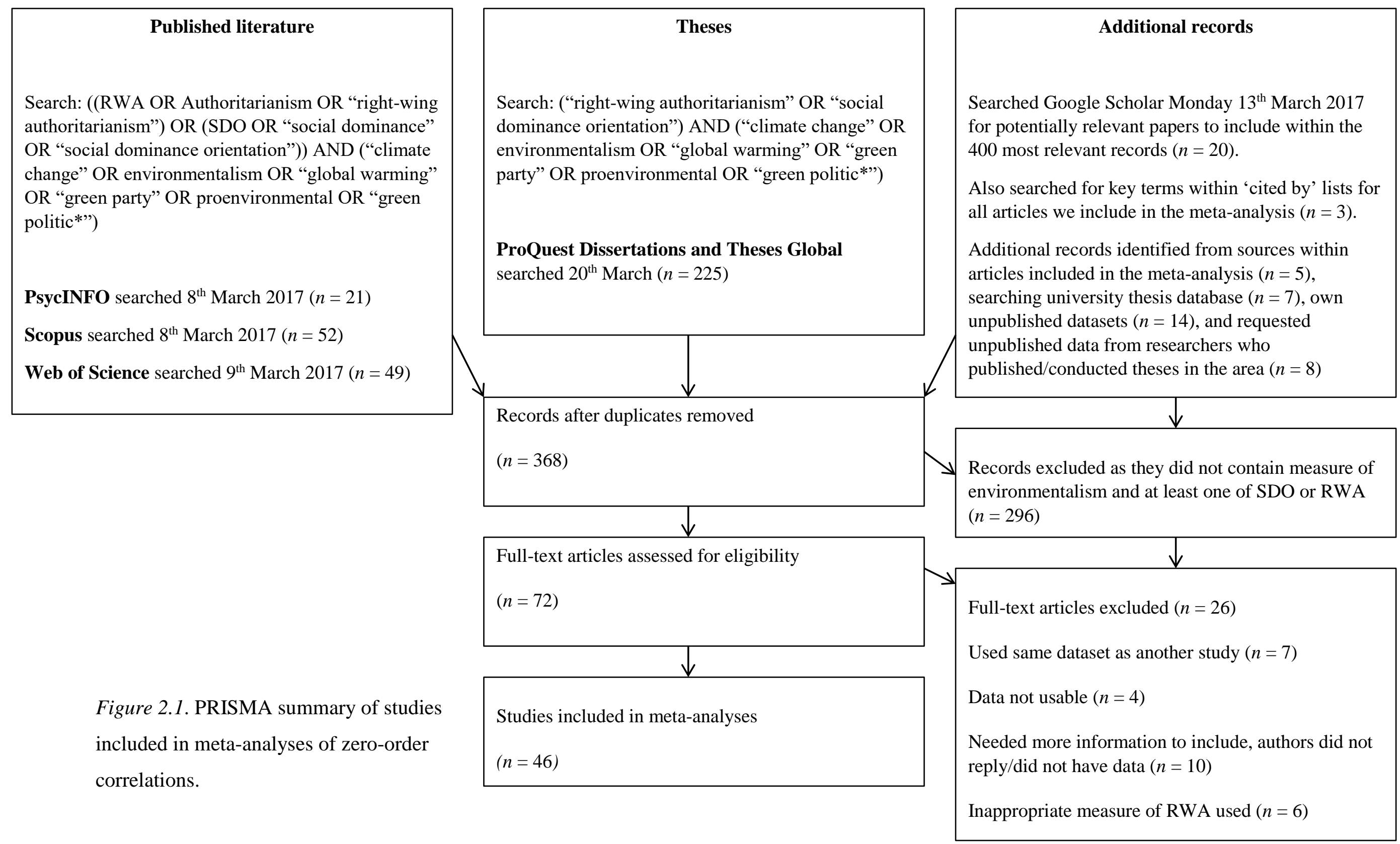




\section{Interrater reliability}

I included a wide range of environmental variables that fit broadly into the six categories listed earlier. I excluded some variables (e.g. support for nuclear power plants or fracking, endorsing environmental reasons for going vegetarian) as these were less direct tests of environmental attitudes, and were judged to not fit the main themes that I created for categorizing the variables. I also wanted to ensure that all correlations were in a pro-environmental direction, so that they could be combined. For example, associations with climate change belief were left unchanged, but associations with climate change denial were reversed.

After initial coding and construction of the coding scheme, I gave my supervisor the environmental measures included in each study and the coding scheme. He coded the variables to fit the established coding scheme (see Table 2.1) with a good level of agreement ( $88.2 \%$ agreement). Cohen's kappa, a measure of interrater agreement that takes agreement by chance into account, demonstrated near perfect agreement in our coding of environmental variables, $\kappa=.85(95 \% \mathrm{CI}[.79, .91]), p<$ .001 (by Landis \& Koch's standards; 1977). We then discussed disagreements, and used the code we both agreed on to separate the variables into the correct metaanalyses. 
Table 2.1.

Coding schedule used to categorize environmental variables.

\begin{tabular}{ll}
\hline Code & Category description \\
\hline $\begin{array}{l}\text { 1. Climate change } \\
\text { belief }\end{array}$ & $\begin{array}{l}\text { Belief in climate change or knowledge about it (e.g. how } \\
\text { much temperature will rise; reverse: climate change denial). } \\
\text { Includes belief that humans are causing climate change (or } \\
\text { that climate change is natural), and shouldn't say otherwise }\end{array}$ \\
2. Support for action & $\begin{array}{l}\text { Willingness to act on climate change, assume responsibility } \\
\text { for the problem and support policy/interventions that } \\
\text { mitigate climate change, including punishment for polluters. }\end{array}$ \\
& $\begin{array}{l}\text { Pro-environmental behavioural intentions or actual } \\
\text { behaviours/activism. Believe we should act soon and try to } \\
\text { reduce emissions (reverse: think government action on } \\
\text { climate change is not needed or exaggerated) }\end{array}$
\end{tabular}

3. Environmentalist threat

4. Green politics

5. Pro-environmental attitudes

6. Climate change anxiety
Attitudes towards environmentalists or fear of environmental activists. Seeing action on climate change as a burden, costly and unnecessary/exaggerating the issue (reverse: support for pro-environmental groups or environmental movement)

\section{Support/vote for the Green party}

New environmental paradigm, value the environment and see benefit of acting on climate change and preserving the environment, emotional connection with nature, feel morally obligated to act (reverse: nature dominance, utilization of nature, apathy)

Perceive climate change/pollution/environmental degradation as threatening/worrying (reverse: think climate change is unlikely to happen to them/now/psychological distance)

\section{Overview of analysis}

I used Comprehensive Meta-Analysis software (CMA; version 2.2.064; Borenstein et al., 2009) to conduct the meta-analyses. Briefly, the first pair of metaanalyses included averages of the environmental variables for each sample with SDO, and separately RWA, and also included tests of potential moderating variables. In the second set of meta-analyses, I entered the associations between SDO, and separately RWA, and the six categories of environmental variables into twelve smaller metaanalyses. In the next sections, I discuss how researchers assess publication bias, test 
whether heterogeneity is present in the corpus of studies included in a meta-analysis, and identify moderating variables.

Publication bias. Problematic for meta-analyses, and the broader literature on a given topic, is that positive results (i.e. in this case, those showing a significant relationship) are more likely to be published than null results (those showing weak or non-significant associations). This is the 'file drawer problem', and it means that we may be overestimating the effect by including only published studies (Rosenthal, 1979). At its worst, we can imagine publication bias resulting in all published studies being those that show a significant relationship due to Type I error (where the relationship is due to error and does not exist in the real world), whereas all those in larger file drawers show the 'true' null association (Rosenthal, 1979).

My first defense against this problem was to contact researchers who published papers or conducted theses in this area to request unpublished data. I was able to include correlations from 14 unpublished datasets that my supervisor has accumulated. Although these steps reduced the total number of unpublished studies omitted from this research, it is impossible to say how many additional studies on this association remain in researchers' file drawers. For instance, I know of at least ten studies that were omitted from the meta-analysis as I could not access the information needed to include them, and it is likely that more studies have been conducted by authors who did not respond or were not included in my emails to request unpublished work. To fully explore this potential problem, I performed several tests to examine the extent to which this remaining publication bias may have affected the results.

I first conducted a visual examination of the funnel plots from each main meta-analysis. The funnel plot gives a graphical depiction of the effect sizes included in each meta-analysis, plotted by their sample size. When publication bias is not an issue, the funnel plot has an even distribution of smaller studies on either side of the plot (Borenstein et al., 2009). This judgement is based on smaller studies because these tend to have more variation from sampling error, and therefore a greater range of effect sizes (Borenstein et al., 2009). However, interpreting funnel plots visually is subjective and potentially subject to bias (Simmonds, 2015), thus to confirm my assessment I supplement visual inspection with several formal statistical tests. 
Duval and Tweedie's (2000) trim and fill analysis works to 'fill the gaps' of the funnel plot, so if the distribution of studies is asymmetric, the test imputes the 'missing' studies. Put simply, the more studies the test imputes and the more this alters the results of the meta-analysis, the more likely it is that publication bias is present, and findings may change if some studies from the 'file drawer' are uncovered and included. When the funnel plot is symmetrical, and therefore publication bias is less likely, no studies will be imputed, and the results of the meta-analysis remain unchanged.

Egger's regression intercept analysis is another test for funnel plot asymmetry, providing evidence for publication bias when it is significant; although when heterogeneity is present, more weight should be placed on inspection of the funnel plot as Egger's test has low power under these circumstances (Simmonds, 2015). Finally, Cooper's fail-safe $\mathrm{N}$ tells the researcher the number of additional studies evidencing null results necessary to reduce the mean correlation to non-significance. Put simply, we can have more confidence that our findings are not subject to publication bias with larger $\mathrm{N}$, but if our results could be reduced to non-significant with only a few more studies added, then it is likely that publication bias is present and problematic (e.g. Rosenthal, 1979).

Detecting variability. I assessed heterogeneity, which is the extent to which there is variability in the correlations included in the meta-analysis above and beyond the amount of variation expected by chance, using the Q statistic and the $I^{2}$ index. As Huedo-Medina, Sánchez-Meca, Marín-Martínez, and Botella (2006) explain, the Q statistic tells us whether there is heterogeneity present, or if the set of studies included in the meta-analysis are homogenous. The Q statistic can be used to justify a choice of statistical model to run the meta-analysis: with no significant heterogeneity (a nonsignificant Q statistic), a fixed effects model is used, whereas when the Q statistic is significant, indicating significant heterogeneity, the researcher ought to use a random effects model (Huedo-Medina et al., 2006).

While the Q statistic identifies if heterogeneity exists, Higgins and Thompson (2002) developed the $I^{2}$ index to describe the impact of the heterogeneity. It is interpreted as a percentage of variability over and above that expected by withinstudy variability (Huedo-Medina et al., 2006). Higgins and Thompson proposed that 
$I^{2}$ values of approximately 25 (meaning $25 \%$; interpreted as quarter of the total variability caused by 'true' variability) are used to represent low heterogeneity, $I^{2}=$ $50(50 \%)$ medium, and $I^{2}=75(75 \%)$ as high heterogeneity.

Results of different studies usually show some variability due to within-study variation, which comes from sampling error. Because we ensure our samples are independent, we expect some variability owing to the use of different samples. A portion of the variability is also due to between-study variability, which arises from actual differences in population effect sizes. Examples of between-study variability include differences in the sample characteristics (e.g. age, gender, student versus general population sample), variations in the study design (e.g. online versus in lab survey, measures used).

Some sources of variability are predictable and expected, and therefore we can take steps to limit the bias they might introduce, or help explain the variability. For example, because I expect that some of the variability in the studies included in the meta-analysis will be due to between-study variability (over and above within-study variability, which is always present), I use a random effects model to take these potential sources of variability into account (Erez, Bloom \& Wells, 1996). Furthermore, I included a range of potential sources of variability as moderating variables to test their effects and help explain the variability.

Both fixed- and random-effects models assign greater weight to studies with larger sample sizes, as they tend to be more precise than smaller studies. While a fixed-effect model works under the assumption that variance in effect sizes is due to sampling variation, the random-effects model acknowledges two sources of potential variance: both sampling variation and between-study variation, and hence give relatively less weight to studies with larger sample sizes than the fixed-effects model. I therefore based estimations on random-effects models, as they are more conservative than fixed-effects models, and account for the expected variance between studies (Sutton \& Higgins, 2008).

Moderator analysis. One way to explain significant heterogeneity in a metaanalysis is through examining the effects of moderator variables (e.g. Huedo-Medina et al., 2006). To assess whether a variable is a significant moderator, such as population type, the overall meta-analysis is first conducted with all studies included 
except those without a defined population type (e.g. where this information is unavailable). Next, the meta-analysis is recomputed while testing for the mean correlation in student samples, and separately in general population samples, effectively conducting two new meta-analyses at once in CMA. The Q values for the meta-analyses on each sample are subtracted from the overall meta-analysis, to obtain a value called the between-level Q: the portion of the overall variability that the moderator explains. When the between-level Q is significant, this means that the moderating variable explains a significant portion of the variability across studies.

Similarly, for a continuous moderator (e.g. proportion of the sample that is female), CMA computes a meta-regression, which points to a significant moderator when the slope is significant. For meta-regression, CMA can base results on either a fixed- or random-effects models. I use the unrestricted ML method for the metaregressions.

A note on family-wise error rates. When conducting multiple statistical tests, the risk of finding significant results due to Type 1 error, where the true effect size is actually not significant (hence, a false positive), becomes more likely (e.g. Tukey, 1949). The risk of finding Type 1 errors increases exponentially with more statistical tests (Polanin, 2013). Because meta-analysis relies on significance testing, the technique also may be susceptible to family-wise error rates, though there is no consensus on this, and no clear solution (Borenstein et al., 2009).

The first pair of meta-analyses, between SDO, RWA, and environmentalism overall, involve just two statistical significance tests each: one for the average effect size, and the other for heterogeneity ( $Q$ test). Because there is not an issue with family-wise error rates in these analyses, I set the $p$-value to the conventional $\alpha=.05$ level. That is, I will reject the null hypothesis (that there is no relationship between the variables) so long as the p-value is below .05, representing at most a $5 \%$ chance that I accept a result as significant that is instead due to chance.

I am conducting multiple tests of statistical significance both when I test for the possible effects of moderating variables, and when I separate the effect sizes to conduct smaller meta-analyses based on the type of environmentalism measured. Therefore, in my moderation analyses, which tested for the potential influence of five moderating variables, add an additional 10 significance tests to each meta-analysis, 
and a further 12 from my split meta-analyses of six separate categories environmentrelevant variables.

To correct for the possibility of erroneously rejecting the null hypothesis (as in a Type 1 error), several procedures have been created to adopt a more conservative $p$ value outside of meta-analysis. Perhaps the most common of these methods, the Bonferroni correction, divides the $p$-value by the number of significance tests conducted. Therefore, for a series of analyses with ten additional tests for example, the alpha level (which was .05) is divided by ten $\left(\alpha_{\text {corrected }}=.05 / 10=.005\right)$. Polanin (2013) argues that this correction is too conservative. While it would work to decrease the likelihood of Type 1 errors, it would also unfairly increase the chances of making a Type 2 error: where we instead risk accepting an incorrect null hypothesis, and miss acknowledging an effect that actually does exist (i.e., a false negative).

Despite methods existing outside of meta-analysis, there is no clear guidance on how to control for family-wise error rates within meta-analysis. Borenstein et al. (2009) advocate for a decrease of the critical $p$-value from .05 to .01. This new thresholds represents a one percent chance of falsely rejecting the null hypothesis, thus is more conservative than the conventional alpha level. Though their recommendation comes without explanation, it does fit typical suggestions to either take .05 or .01 as appropriate critical values. However, these guideline were made for medical research, when the consequences of a false positive might be more devastating than false negatives. On balance, I argue that neither solution represent a good compromise between guarding against Type 1 error, while also not going as far as to increase the risk of Type 2 errors. I consistently use random-effects models throughout the analyses in this chapter, despite recommendations from other researchers turn to fixed-effects models in order to increase the sensitivity of the test (and hence, the likelihood of finding significant results; Helmus, 2017, personal communication). Because of the low power of random-effects models, I am already at a greater risk of making Type 2 rather than Type 1 errors.

Ultimately I therefore decided the best option was to rely on the $p$-value of .05 to evaluate the statistical significance of the findings from all statistical tests in the meta-analyses and use random-effects models where appropriate. Furthermore, following the recent shift to examine confidence intervals rather than significance 
tests (e.g. Cumming, 2013), I also consider confidence intervals that do not include zero to indicate a noteworthy effect, and when comparing estimates of SDO and RWA, non-overlapping confidence intervals to indicate statistically different results.

\section{Results}

I identified a total of 33 studies using 53 independent samples to include in our main meta-analysis on SDO and environmentalism, and 32 studies using 53 independent samples for the meta-analysis on RWA and environmentalism. In this section, I discuss the results of the meta-analyses: firstly with all environmental variables included for each ideological correlate, and then with environmentalism separated into climate change belief, support for action, environmentalist threat, Green politics, pro-environmental attitudes, and climate change anxiety (refer to Table 2.1 for descriptions of these category definitions).

\section{Overall meta-analyses}

The overall meta-analyses of the associations between environmentalism and SDO, and separately RWA, were conducted using the average correlation of this relationship for each unique sample. As predicted in $\mathrm{H} 1$, both mean correlations were significant, with SDO a slightly stronger correlate $(r=-.294,95 \%$ CI [-.322, -.266], $p$ $<.001)$ than RWA $(r=-.261,95 \%$ CI $[-.304,-.217, p<.001)$. As shown in Figure 2.2, the confidence intervals for these estimates overlap, thus SDO was not a statistically stronger correlate than RWA, contrary to my second hypothesis. Instead, this finding suggests that SDO and RWA are comparable correlates of environmentrelevant variables. 


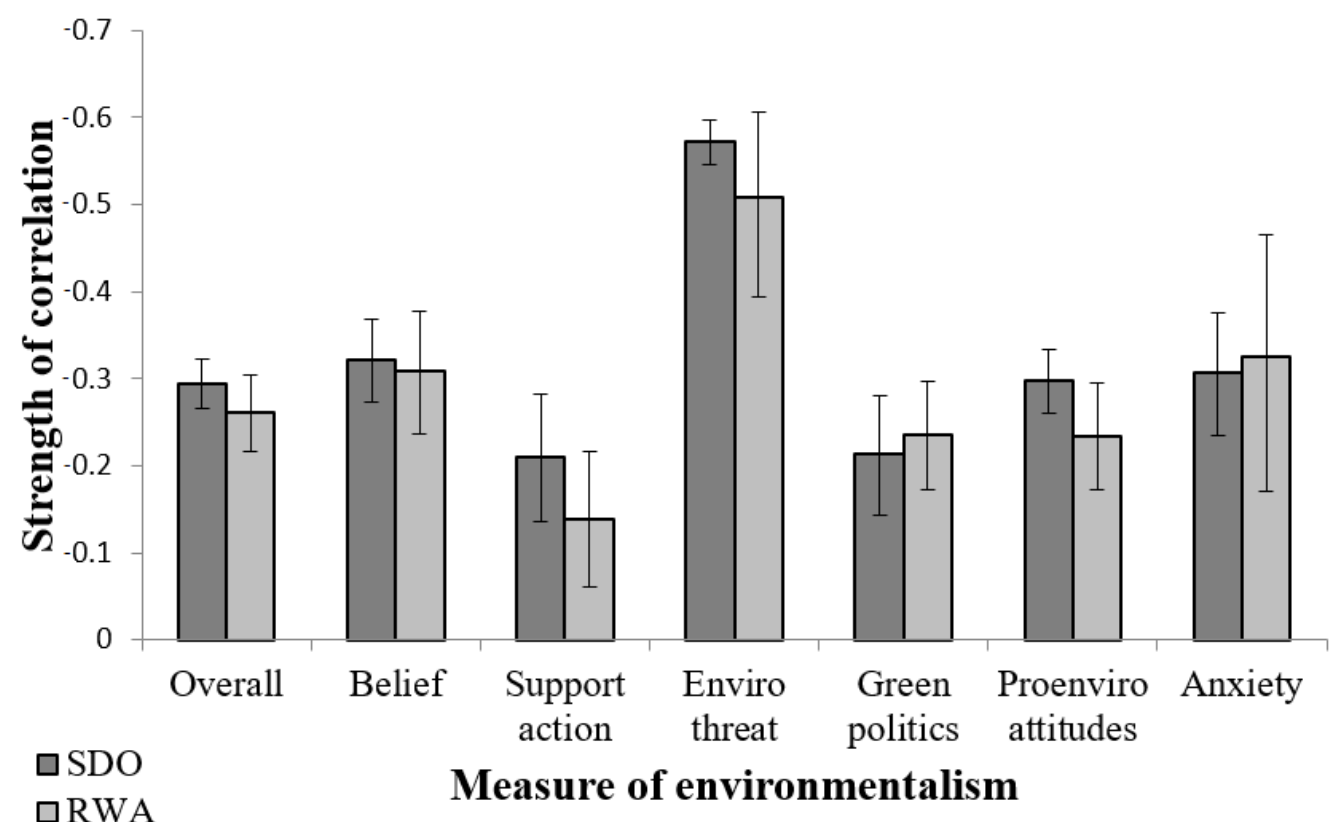

Figure 2.2. Mean meta-analytic correlation between ideological variables $(\mathrm{SDO}=$ social dominance orientation, $\mathrm{RWA}=$ right-wing authoritarianism $)$ and environmental variables.

Publication bias. While I did take steps to reduce the potential impact of publication bias, such as including correlations from unpublished manuscripts and datasets, it is still an important step to test if publication bias is a problem for the meta-analyses. Visual examination of both funnel plots (see Figures $2.3 \& 2.4$ ) show roughly even distributions of small studies on either side of the plots. Supporting this informal test, for SDO, Egger's regression is non-significant $(\mathrm{t}(51)=.36, p=.359)$, trim and fill analysis imputed zero studies, leaving the mean correlation and confidence intervals for the combined studies unchanged, and Cooper's fail safe $\mathrm{N}$ requires that 26,016 studies with null results are included to undermine these results.

The meta-analysis for RWA failed the Egger's regression test $(\mathrm{t}(51)=1.87, p$ $=.034)$, however Begg's test was not significant $(p=.128)$, the meta-analysis was not subject to any changes in the trim and fill analysis, and obtained a fail safe $\mathrm{N}$ of 17,460. To further probe whether publication bias could be an issue for the RWA meta-analysis, I reanalyzed the data with publication status as a moderator (i.e. published versus unpublished effect sizes). The strength of the association was not 
influenced by publication status $(\mathrm{Q}(1)=.56, p=.455)$. Taken together, these indices, in conjunction with the visual examination of the funnel plots below, suggest that publication bias was not a problem for either meta-analysis.

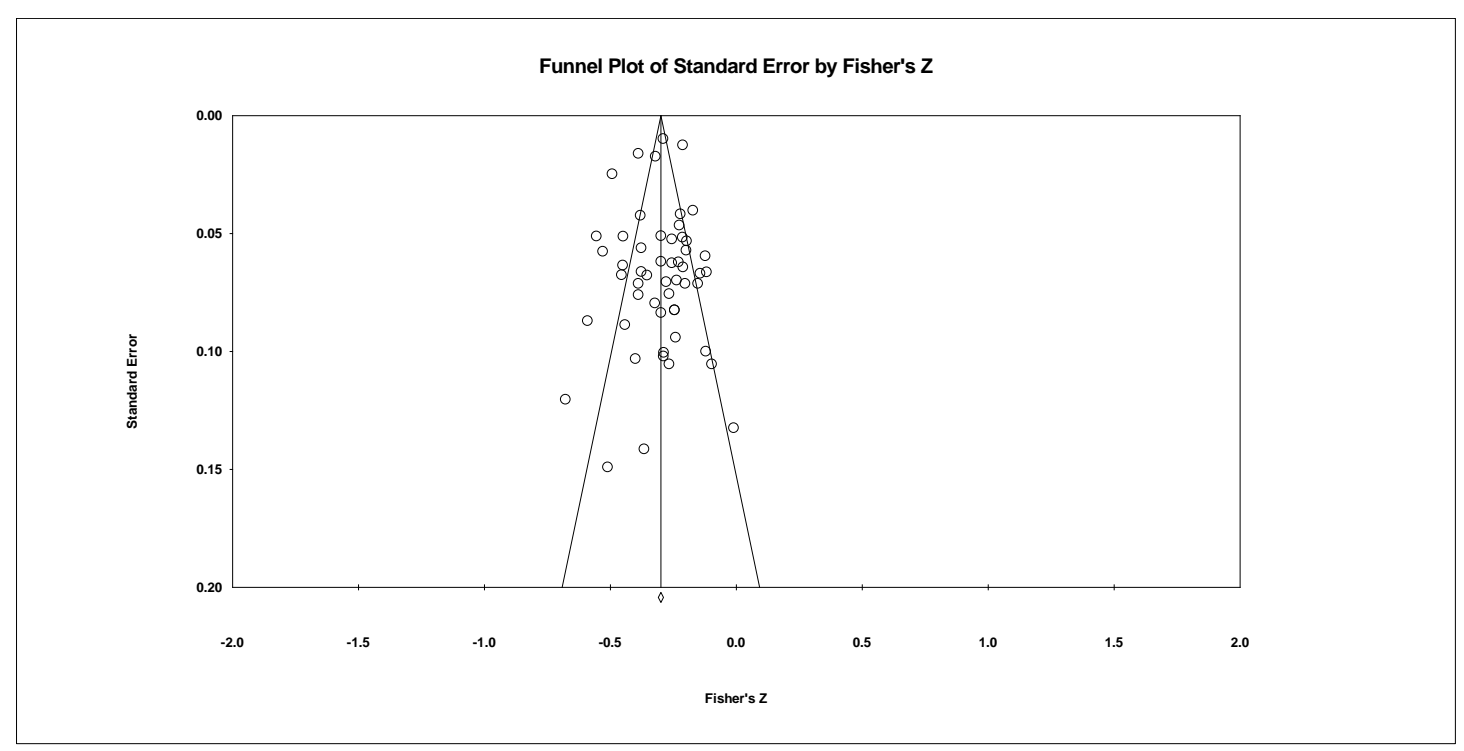

Figure 2.3. Funnel plot showing the distribution of studies and effect sizes for the meta-analysis between SDO and environmentalism.

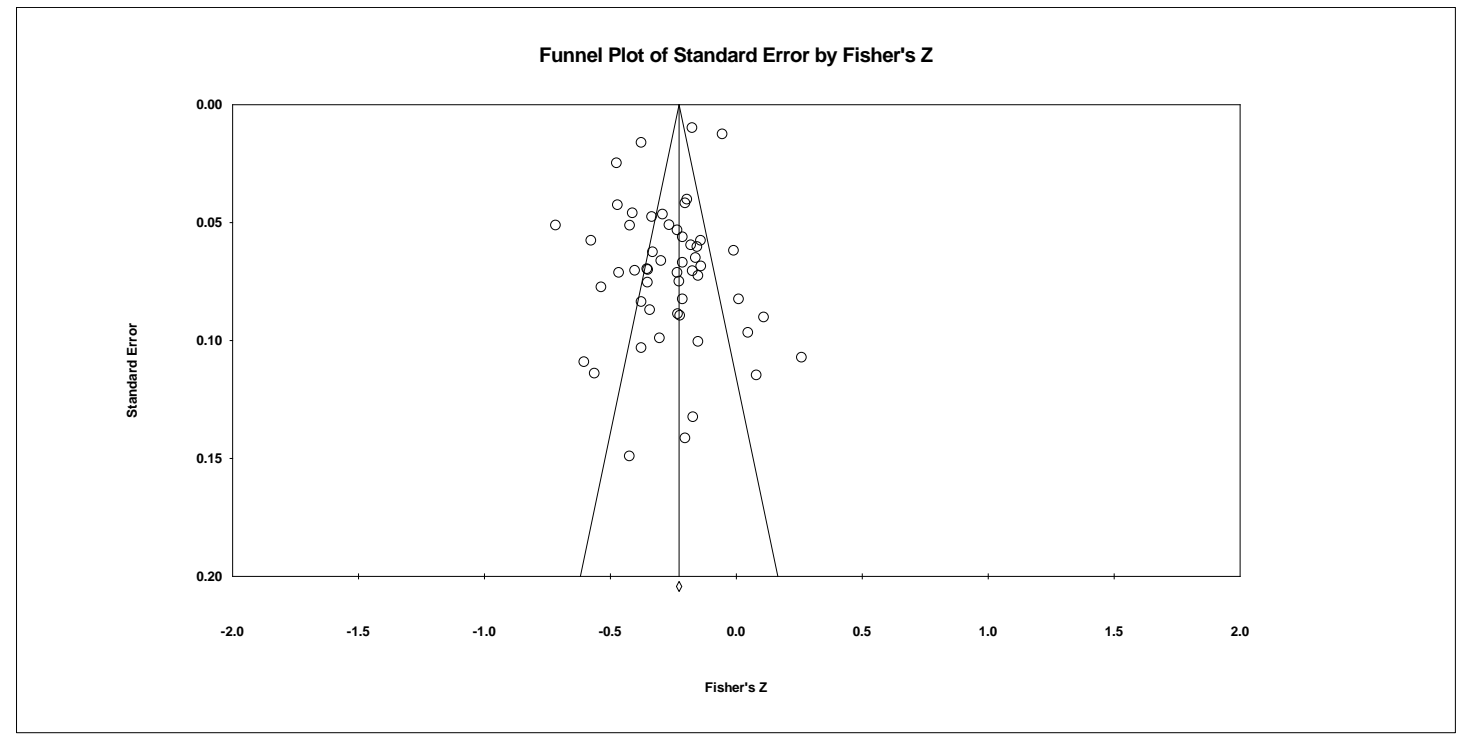

Figure 2.4. Funnel plot showing the distribution of studies and effect sizes for the meta-analysis between RWA and environmentalism.

Variability. I observed significant heterogeneity for both $\mathrm{SDO}(\mathrm{Q}(52)=$ $\left.311.75, p<.001 ; I^{2}=83.32\right)$ and RWA $\left(\mathrm{Q}(52)=759.75, p<.001 ; I^{2}=93.16\right)$. These 
values were similar to those reported in Hornsey et al.'s (2016) meta-analysis for the associations between climate change belief and political affiliation $\left(I^{2}=72.06\right)$ and political ideology $\left(I^{2}=91.43\right)$.

Moderation analysis. I explored the effect of two categorical moderator variables that I suspected, based on examination of previous studies' findings, to have some influence on the strength of the relationships, and therefore having the potential to help explain some of the variability present across studies. The first moderator is scale type, including full versus shortened SDO scales (e.g. Pratto et al., 1994), the $\mathrm{SDO}_{7}$ (e.g. Ho et al. 2012), and other measures of SDO, and for RWA the full and shortened RWA scales (e.g. Altemeyer, 1998), Zakrisson's (2005) measure, and the ACT scale (Duckitt, Bizumic, Krauss \& Heled, 2010). I also considered population type (general population versus student sample). Also from previous findings of differences in environmentalism and SDO by gender (e.g. Jylhä et al., 2016), I consider the effect of gender on these relationships by including the ratio of males to females as a continuous moderator.

For the SDO-environmentalism association, scale type was not a significant moderator $(\mathrm{Q}(3)=6.47, p=.091)$, meaning the overall strength of the relationship was not dependent on how SDO was measured. I had expected that the association would be stronger when SDO was measured using a full scale compared to when a shortened scale was utilized, so this finding did not support H3. Instead, the relationship was comparable when measured using the full SDO scale $(r=-.293,95 \%$ CI [-.343, -.242]), a shortened version of this ( $r=-.270,95 \%$ CI [-.317, -.221]), Ho et al.'s $\mathrm{SDO}_{7}$ scale $(r=-.373,95 \% \mathrm{CI}[-.437,-.304])$ or other measures, such as egalitarianism ( $r=-.269,95 \%$ CI [-.341, -.194]). While it appears that the relationship is substantially stronger when SDO is measured using the most recent measurement tool, the $\mathrm{SDO}_{7}$, the difference is not significant (indicated by the overlapping confidence intervals with other measures), though this could be due to low power (only six samples employed this measure).

Providing support for $\mathrm{H} 4$, population type was a significant moderator of the relationship between SDO and environmentalism $(\mathrm{Q}(1)=9.86, p=.002)$, with a slightly stronger association in general population samples $(r=-.335,95 \%$ CI [-.374, 
$-.294])$ as compared to student samples $(r=-.247,95 \%$ CI [-.331, -.202]). I discuss this finding in more detail later.

Next, I considered the effect of gender as a continuous moderator. For the meta-regression with sample gender ratio, I employed a random-effects metaregression using an unrestricted maximum likelihood model. Contrary to hypothesis 5, which predicted a stronger association in samples with greater proportion of males, the gender composition of the sample did not influence the association $(\mathrm{Q}(1)=0.01, p$ $=.908$ ). Therefore, the hypothesis that the association between SDO and environmentalism was rejected.

For RWA, there was no effect of population type $(\mathrm{Q}(1)=.16, p=.689)$ or scale type $(\mathrm{Q}(3)=2.19, p=.533)$, findings that do not support H4. Finally, as with SDO, the association between RWA and environmentalism was not influenced by gender (random-effects $\mathrm{Q}(1)=.00, p=.951)$.

Overall, therefore, these results support my first prediction that both SDO and RWA would be significant correlates of environmentalism, although there was no significant difference in their predictive abilities, when I had expected SDO to exhibit a stronger relationship. Contrary to expectations, scale type was not a significant moderator for either ideological variable, which is fortunate as such a finding would warrant caution for all existing studies and datasets that measure SDO and RWA using shortened scales. I found partial support for my fourth hypothesis: the association between SDO and environmentalism varied depending on whether a general population or student sample were used, but for RWA, the mean correlation held across sample types. I did not find support for gender as a continuous moderator, suggesting instead that the associations between SDO, RWA and environmentalism hold regardless of the gender composition of the samples.

\section{Separate meta-analyses by type of environmental variable}

Next, I conducted a series of separate meta-analyses for each ideological variable according to our six categories of environmental variables. Where more than one correlation was reported for the same sample that fit the same broad environmental category (e.g. belief that climate change was happening, and belief that climate change is caused by humans), I included an average of these correlations to maintain statistical independence (for the example, these fit into the 'Climate change 
belief' category). Here, I report the mean correlations obtained in the meta-analyses, which are also graphed with the findings of the overall meta-analyses in Figure 2.2. Tables 2.2 contain the number of studies included in each meta-analysis, the mean correlation, and heterogeneity indices for these analyses, for SDO and RWA, respectively. For all Q statistics included in the table, $p<.001$. 
Table 2.2.

Results of the meta-analyses of the correlation between SDO, RWA, and six environment-relevant variables.

\begin{tabular}{|c|c|c|c|c|c|c|c|c|}
\hline & \multicolumn{4}{|c|}{ Social dominance orientation } & \multicolumn{4}{|c|}{ Right-wing authoritarianism } \\
\hline $\begin{array}{l}\text { Measure of } \\
\text { environmentalism }\end{array}$ & $\underline{k}$ & Mean $r[95 \% \mathrm{CI}]$ & $\underline{Q}$ & $\underline{\mathrm{I}^{2}}$ & $\underline{k}$ & $\underline{\text { Mean } r[95 \% \mathrm{CI}]}$ & $\underline{Q}$ & $\underline{I^{2}}$ \\
\hline Overall & 53 & $\begin{array}{c}-.29 \\
{[-.32,-.27]}\end{array}$ & 311.75 & 83.32 & 53 & $\begin{array}{c}-.26 \\
{[-.30,-.22]}\end{array}$ & 759.75 & 93.16 \\
\hline Belief in climate change & 24 & $\begin{array}{c}-.32 \\
{[-.37,-.27]}\end{array}$ & 301.18 & 92.36 & 21 & $\begin{array}{c}-.31 \\
{[-.38,-.24]}\end{array}$ & 602.84 & 96.68 \\
\hline Support for action & 15 & $\begin{array}{c}-.21 \\
{[-.28,-.14]}\end{array}$ & 173.35 & 91.92 & 13 & $\begin{array}{c}-.14 \\
{[-.22,-.06]}\end{array}$ & 93.40 & 87.15 \\
\hline Environmentalist threat & 3 & $\begin{array}{c}-.57 \\
{[-.60,-.55]}\end{array}$ & 1.93 & 0 & 6 & $\begin{array}{c}-.51 \\
{[-.61,-.39]}\end{array}$ & 71.07 & 92.97 \\
\hline Green politics & 14 & $\begin{array}{c}-.21 \\
{[-.28,-.14]}\end{array}$ & 83.34 & 84.40 & 19 & $\begin{array}{c}-.24 \\
{[-.30,-.17]}\end{array}$ & 115.63 & 84.43 \\
\hline Pro-enviro attitudes & 35 & $\begin{array}{c}-.30 \\
{[-.33,-.26]}\end{array}$ & 248.27 & 86.31 & 29 & $\begin{array}{c}-.23 \\
{[-.29,-.17]}\end{array}$ & 586.50 & 95.23 \\
\hline Climate change anxiety & 6 & $\begin{array}{c}-.31 \\
{[-.38,-.23]}\end{array}$ & 21.46 & 76.70 & 4 & $\begin{array}{c}-.33 \\
{[-.47,-.17]}\end{array}$ & 27.26 & 92.66 \\
\hline
\end{tabular}


In each of the meta-analyses, SDO and RWA are significant correlates. Furthermore, all correlations are negative, consistently showing that the more that people endorse socially dominant or right-wing authoritarian attitudes, the less proenvironmental they are, as expressed by the lower likelihood of reporting belief in climate change, supporting action on climate change, supporting or voting for Green political parties, or expressing pro-environmental attitudes. Instead, they are more likely to feel threatened by the environmental movement, and are less anxious about the threat of climate change itself.

In all cases, the confidence intervals for the mean correlations between SDO and environmentalism and RWA and environmentalism overlap. Therefore, for each of the six types of environmental variables, SDO and RWA show comparable correlations. I had expected SDO and RWA to differentially predict some environmental variables, predicting SDO would be more strongly related to climate change belief, support for action on climate change, pro-environmental attitudes and climate change anxiety, and RWA to relate more strongly to Green politics. These expectations were consistent with the direction of the associations, however I conclude from these findings that there are no significant differences in the predictive ability of SDO and RWA. Instead, they are comparable correlates of environmentalism overall, and each of the six aspects of environmentalism I identified.

Interestingly, the mean associations for both SDO and RWA are significantly stronger with environmentalist threat than for any other category of environmentalism, based on non-overlapping confidence intervals. This might indicate that the aspect or target of environmentalism that Social Dominants and Authoritarians most strongly oppose is environmentalists themselves, instead feeling that action on climate change is a burden. However, these findings should be interpreted with caution, as this category included correlations from the fewest number of samples.

\section{Comments}

The aim of this first study of my thesis was to conduct a wide review of the literature to date on the ideological correlates of environmentalism, specifically social dominance orientation (SDO) and right-wing authoritarianism (RWA). To do this, I 
built on work conducted by Hornsey et al. (2016) on the correlates of climate change belief. Hornsey et al. demonstrated that while education and knowledge about climate change might be somewhat related to belief in climate change, political affiliation is a stronger correlate. Those who intend to vote for left-wing parties are more likely to believe that climate change is happening, and that humans contribute to the problem. As research indicates that SDO and RWA are strongly related to, if not foundational for, political ideology, I expand on Hornsey et al.'s work to show that SDO and RWA are significantly related to a range of aspects of environmentalism.

Summarizing the link reported in 53 independent samples each, both SDO and RWA are significant correlates of environmentalism. While the association with environmentalism overall was slightly weaker, my meta-analyses yielded mean correlations with climate change belief $\left(r_{\mathrm{SDO}}=-.32 ; r_{\mathrm{RWA}}-.31\right)$ that were comparable to those reported by Hornsey et al. (2016) on the associations between climate change denial and political affiliation $(r=.30)$, objective knowledge on climate change ( $r=$ $.25)$, and both public $(r=.25)$ and private $(r=.32)$ pro-environmental intentions.

While I showed that SDO tends to have a slightly stronger relationship with environment-relevant variables than RWA (except when environmentalism is indexed by support for Green politics or climate change anxiety), the analyses presented here do not show this difference to be statistically significant - instead, the associations are comparable. Indeed, they demonstrate that ideology relates to a range of antienvironmental sentiments, thus is important for understanding more than just climate change belief.

Another important finding comes from the moderation analyses: Despite my prediction, there is insufficient evidence to conclude that scale type moderates the relationship between either of our ideological variables and environmentalism. Instead, the associations between SDO, RWA, and environmentalism hold regardless of whether full, shortened, or other scale measures are employed. In large-scale surveys (including some of those reported in this thesis), it is sometimes necessary to include only a subset of items to measure a construct, so that more constructs can be measured within the same questionnaire without drastically increasing participant fatigue (and therefore increasing potential for errors; Ben-Nun, 2008). Based on the results of my meta-analyses, it is likely that using shortened scales (at least to measure 
SDO, RWA, and their association with environment-relevant variables), significantly effects results. One caveat is that I did find that alternative measures of authoritarianism showed a large amount of variability (see footnote on page 42), indicating that they were perhaps not measuring the same construct as RWA scales. Future research might usefully compare these measures more directly to determine how much overlap they have with the RWA construct captured by validated scales.

The moderation analyses also revealed that the relationship between SDO and environmentalism is dependent on the population sampled. There is a stronger relationship between SDO and environmentalism for general population samples than for student samples. This is unsurprising given that students tend to score differently on ideological variables, for example Roccato and Ricolfi (2005) found that the association between SDO and RWA is typically weaker in student samples, and thus recommended drawing from general population samples to measure these constructs. Despite this, researchers continue to collect data from student samples, as they are often more easily accessible. While I found that using student samples may underestimate the relationship between SDO and environmentalism, for RWA, the relationship is as strong in student populations as in general population samples. Therefore I urge caution, at least in the case of SDO, about drawing conclusions about the variable's association with environmentalism based solely on research recruiting from student populations.

There are some reports in the literature of gender differences in the association between ideology and environmentalism (e.g. the 'conservative white male effect', where conservative males tend to be less convinced about climate change compared to liberals and females; McCright \& Dunlap, 2011). However, I did not find evidence to suggest that the relationships between SDO, RWA and environmentalism were affected by the gender composition of the samples. This is perhaps consistent with Hornsey et al.'s (2016) meta-analysis, which showed a minimal relationship between climate change belief and gender $(r=.029)$. It is also possible that I did not have enough power to detect the relationship in the random-effects model, which is typically underpowered (Lipsey, 2003). Therefore, more thought might need be given as to whether and why gender could be a correlate of environmentalism, and under what conditions. 
One take away message from the series of separate meta-analyses is that SDO and RWA are consistently related to the multiple dimensions of environmentalism: all are associated with lower pro-environmentalism. From a measurement perspective, our findings indicate that while utilizing multiple measures of environmentalism might demonstrate subtle differences in the strength of the relationship, they all show roughly equivalent (and indeed, at least not significantly different) associations with SDO and RWA.

One major limitation of this study is that I have examined these associations using correlations, and therefore cannot make any claims about causal relationships between SDO, RWA, and environmentalism, or any claims about the effect of the combination of SDO and RWA on environmentalism. Furthermore, correlations do not allow for the examination of the unique relationship between environmentalism and each ideological variable. I aim to address this second limitation in Study 1B by meta-analyzing the relationship between each ideological variable and environmentalism while controlling for the effect of the other. 


\section{Study 1B}

\section{Meta-analyses of the unique effects of ideology on environmentalism}

\section{Introduction}

The take home message of Hornsey et al.'s (2016) synthesis of research on the predictors of climate change belief was clear: Being more educated, or more knowledgeable about climate change does not necessarily mean that people are more convinced that climate change is real. Instead, people evaluate the evidence of climate change to the extent that it is consistent with their own values, political loyalties, and ideologies. Recent research has turned to SDO and RWA as important determinants of environmental attitudes (e.g., Häkkinen \& Akrami, 2014; Milfont et al., 2013). In Study $1 \mathrm{~A}$, I reported results of a series of meta-analyses of the research to date, showing that SDO and RWA do indeed relate to lower levels of support for Green political parties and action on the problem, pro-environmental attitudes, and less belief in, and anxiety towards, climate change. Instead, Social Dominants and Authoritarians are more likely to feel threatened by environmental activists and the potential effects of their actions.

Although Study 1A demonstrated that both SDO and RWA are important ideological correlates of environmentalism, it fails to tease apart exactly how much of the relationship between each ideological variable and environmentalism is unique, and how much is attributable to the shared variance between SDO and RWA. As alluded to in the Comments section of Study 1A, research typically finds a weak to moderate association between SDO and RWA (e.g. Altemeyer, 1998; Duckitt, 2001). Further complicating this issue, the relationship between the ideologies is dependent on context.

In a New Zealand context, SDO and RWA tend to correlate moderately as our political system is organised along a single dimension from left to right, with less highly polarised societies showing weak to null associations between the variables (Duckitt, 2001). SDO and RWA similarly predict support for our two main political parties, with those scoring more highly in these ideologies tending to be supportive of 
National (our main conservative party), and tending to oppose Labour (liberal party; Sibley \& Wilson, 2007). Furthermore, endorsement of SDO and RWA relates to lower support for the Green party, our left-wing political party with a strong focus on policies for environmental protection (Sibley \& Wilson, 2007). Further, SDO and RWA interact to predict political ideology in New Zealand with those scoring high on both being the most politically conservative (Wilson \& Sibley, 2013).

Many of the studies included in the first set of meta-analyses $(k=22$ out of 53) were conducted in New Zealand. It is therefore likely that some of the SDOenvironmentalism relationship is explained by the relationship between RWA and environmentalism, and the shared variance that SDO has with RWA, and the same for the relationship between RWA and environmentalism. In Study 1B, I aim to evaluate the unique effect each ideological variable has on environment-relevant variables.

\section{Meta-analyzing the unique effect of SDO and RWA on environmentalism}

Meta-analysis is a useful technique for combining effect sizes across studies to yield an overall effect size that better approximates the true size of the effect in the population. This is achieved by accumulating evidence from existing studies, which all estimate a similar relationship, but also contain some error (Rosendaal \& Reitsma, 2014). Pooling the data yields perhaps a more valid and reliable estimate of the true effect by computing a weighted average of the findings from each study.

Findings from each study included in a meta-analysis are represented by effect size statistics. Meta-analysis software permits a range of effect size statistics, with one such program offering over one hundred formats for entering data, from simple correlations to group differences (Borenstein et al., 2009). In Study 1A, I was interested in the relationships between SDO, RWA, and environmentalism. To investigate this, I included Pearson's correlation coefficients as a measure of effect size for these meta-analyses. Correlation coefficients capture the bivariate relationship between each ideological variables (SDO and RWA, separately) and environmentalism, without controlling for the effects of any other variable. Many published papers include correlation matrices between key variables included in their study, making collecting correlation coefficients from existing research a relatively accessible way to meta-analyze a relationship between variables. 
When there is only one predictor variable in a bivariate regression analysis, the result matches the bivariate (zero-order) correlation between the two variables. Adding a second predictor, as in multiple regression, typically reduces this estimate, resulting in a standardized regression coefficient that is smaller than the correlation coefficient. This is because multiple linear regression analysis partials out the amount of variance each predictor variable explains in the outcome variable: the relationship is smaller as the variance that is explained by the second predictor is taken out, leaving only the unique association between the two variables. Figure 2.5 shows a visual depiction of this. The rare exception to this case is where a suppressor effect occurs (e.g. Velicer, 1968). As this is not the case for the variables discussed here, the issue of suppressor effects is not discussed in further detail.

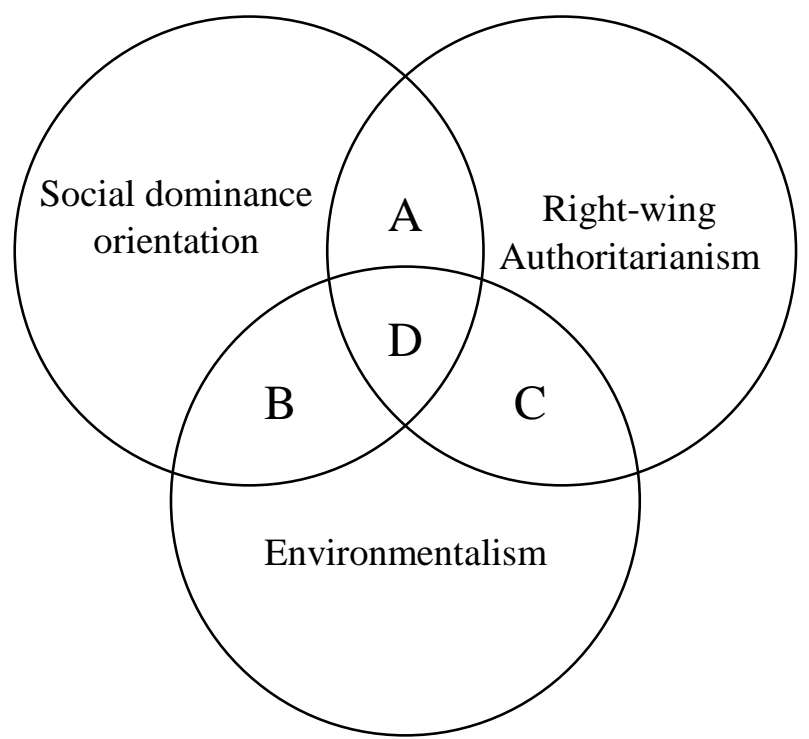

Figure 2.5. Partitioning of the variance explained in a multiple regression analysis, where environmentalism is regressed onto SDO and RWA.

In this figure, the correlation between SDO and environmentalism is represented by sections B and D of the diagram, and between RWA and environmentalism in C and D. Section D represents the association with environmentalism that is accounted for by the shared variance between SDO and RWA. By controlling for each in turn, we can measure the unique association between SDO and environmentalism (section B), and the unique association between RWA and environmentalism (section C). 
Kim (2011) explains that the difference between the regression coefficient and the correlation coefficient depends on the strength of the relationship between the two predictors. When there is a weak to null association between the two predictors (e.g. $r$ $=0.10$ ), the standardized regression slope is almost identical to the correlation coefficient. When variables are more strongly correlated ( $r=.50$ to .70$)$, there is a marked difference in the strength of the regression weight. We know that there is a relationship between SDO and RWA, which is typically weak, though stronger in highly ideologically organized contexts such as New Zealand (see Duckitt, 2001). This means that they likely covary enough to yield weaker associations with environmentalism in their regression coefficients than those represented by the bivariate correlation coefficients.

In order to test which ideological variable explains the most variance in environmentalism (and is therefore the stronger predictor), it is necessary to control for the effects of each variable while examining the relationship to estimate the unique contribution of each variable in predicting environmentalism. This is achieved in multiple regression analysis, when both SDO and RWA are entered as predictor variables, with environmentalism as the dependent variable.

\section{Current study}

In Study 1B, I include only a subset of the samples from Study 1A. This is because I needed to conduct additional analyses on raw data files, and therefore was restricted to only using the 16 full datasets available to me. I calculated the standardized regression coefficients for SDO and RWA as they simultaneously predict environmentalism, and meta-analyzed these results. Following the same rationale as in Study 1A, I expected that both SDO and RWA would explain unique variance in environmentalism, however the association with SDO (while controlling for RWA) would be stronger (H1). I included the significant moderator from Study 1A (sample type), and expected (based on those findings) that population type will moderate the association between SDO (but not RWA) and environmentalism, with a stronger association in general population samples than in student samples (H2).

As well as meta-analysing the association between SDO, RWA, and environmentalism overall, I also ran separate meta-analyses to see how the ideological variables predicted four indices of environmentalism assessed in the data 
sets that were available: Climate change belief, support for action, Green politics, and pro-environmental attitudes. For these smaller meta-analyses, I expected to find a similar pattern of results as in Study 1, with SDO a stronger predictor of climate change belief, support for action, and pro-environmental attitudes, and RWA a stronger predictor of Green politics (H5).

\section{Method}

\section{Sample of studies}

For these analyses, I needed to calculate effect sizes from raw datasets (see below for rationale). This includes 14 independent samples from my supervisor's personal collection, as well as two from a colleague, totaling data from 23,856 participants (for a full list of included studies, see Appendix B). All data were collected in New Zealand, tended to have more female than male participants (except in one case), and used a range of full and shortened SDO and RWA scales (i.e. no alternative measures). Of the 16 , half sampled from the general population, and half from student samples.

\section{Data extraction}

My first step was to decide what kind of measure of effect size to use to test my research question. As explained above, including correlation coefficients is inappropriate to test this new research question, which concerns the unique relationship each variable has with environmentalism, as bivariate correlation coefficients do not control for the effect of other variables.

Multiple regression (as described above) is the most appropriate analysis for this situation, however gaining the right information from the analyses to include regression coefficients as the measure of effect size proves challenging. To include an effect size from a regression analysis in CMA, researchers must include either the standard error of the effect size, or the $95 \%$ confidence interval surrounding the effect size (Borenstein et al., 2009). However, data packages such as SPSS only provide this information for the unstandardized regression slopes (Kim, 2011). Unstandardized regression slopes are often inappropriate to include as effect size estimates in metaanalyses for several reasons. Firstly, in order to combine regression slopes, the researcher must ensure that all predictors are the same across studies, with the same 
predictor variables making up the model (Becker \& Wu, 2007; Nieminen et al., 2013). If different studies control for the effects of different predictor variables, this affects the strength of the relationship between the predictor variable of interest to the metaanalysis and the outcome variable. This is because any additional predictors in the model will reduce the size of the estimate, as the effect of more variables is being controlled for. I avoid this criticism (which could also be leveled at standardized effect sizes) by using raw datasets to calculate this information myself, thus including only my key predictor variables (SDO and RWA) in each regression analysis. A second reason, which is more relevant to the current study, is that raw-scale slopes are usually not on equivalent metrics. Predictor and outcome variables tend to be measured slightly differently across studies, or on different scales, and therefore these estimates cannot be added together using meta-analysis (Becker $\& \mathrm{Wu}, 2007$ ).

Including standardized estimates avoids these issues. This is because they are scale free, meaning that the effect sizes are comparable across studies, and are therefore better candidates to combine using meta-analysis. Standardized regression slopes are also easier to interpret because the variance of standardized estimates equal one, the regression slope is a measure of the number of standard deviations the dependent variable changes for each one standard deviation increase in the predictor variable (Vittinghoff, Glidden, Shiboski \& McCullouch, 2005).

However, standardized estimates (beta weights) are generally reported without their associated standard errors or confidence intervals (Aloe, 2009), likely in part due to common statistical packages such as SPSS not providing this information. To include the standardized estimates in a meta-analysis, I needed to take steps to calculate this additional information. I followed Kim's (2011) method for calculating the standard error for standardized estimates. She begins with the knowledge that the $t$ statistic is equivalent for standardized and unstandardized slopes. The $t$ statistic is the estimate (either the unstandardized $\mathrm{B}$ or standardized beta weight $/ \beta$ ) divided by the standard error for that estimate (i.e., $t=\beta / \mathrm{SE}_{\beta}$ and $t=\mathrm{B} / \mathrm{SE}_{\mathrm{B}}$ ). Using algebra to transform this formula, for each predictor in the regression model, the standard error for the beta weight is obtained by dividing the standardized regression coefficient by the corresponding $t$ statistic $(\mathrm{SE}=\beta / t$ ). The SPSS output for multiple regression provides both beta weights and the associated $t$ statistic, so I used SPSS version 23 (IBM, 2017) to obtain this information for the meta-analyses. 


\section{Overview of analyses}

As in Study 1A, I analysed the data using Comprehensive Meta-Analysis software (CMA; version 2.2.064; Borenstein et al., 2009). When more than one index of environmentalism was included in the same dataset, I maintained independence of samples by averaging the beta weight and standard error for each environmental variable included in the dataset, so that I included only one effect size per sample. CMA does not have a dedicated option for entering standardized regression coefficients, recommending instead that regressions are entered using the generic 'Point estimate' option for this type of effect size (Borenstein et al., 2009). I entered these and their accompanying standard errors (rather than confidence intervals), after using Kim's (2011) formula to calculate the standard errors from the information provided by the SPSS output for each regression.

I used the same indices to assess variability and the potential influence of moderating variables as detailed in Study 1A, and included a test for the moderating effects of sample type (general population versus student). I used random effects models, supported by the significant variation across studies in both main metaanalyses (SDO: $\mathrm{Q}(15)=46.83, p<.001, I^{2}=67.97$; RWA: $\mathrm{Q}(15)=162.37, p<.001$, $\left.I^{2}=90.76\right)$.

\section{Results}

\section{Testing the strength of the predictors}

In the first set of meta-analyses, I entered an averaged beta weight from each study on the relationship between SDO and environmentalism (while controlling for RWA), and between RWA and environmentalism (while controlling for SDO). As shown in Figure 2.6, both SDO and RWA are significant independent predictors of environmentalism (SDO: $\beta=-.168,95 \%$ CI [-.203, -.132], $p<.001$; RWA: $\beta=-.193$, $95 \%$ CI [-.252, -.135], $p<.001)$. Furthermore, as evidenced by the overlapping confidence intervals of these estimates, the results suggest that SDO and RWA have comparable unique associations, rather than SDO being a stronger predictor (as I predicted). 


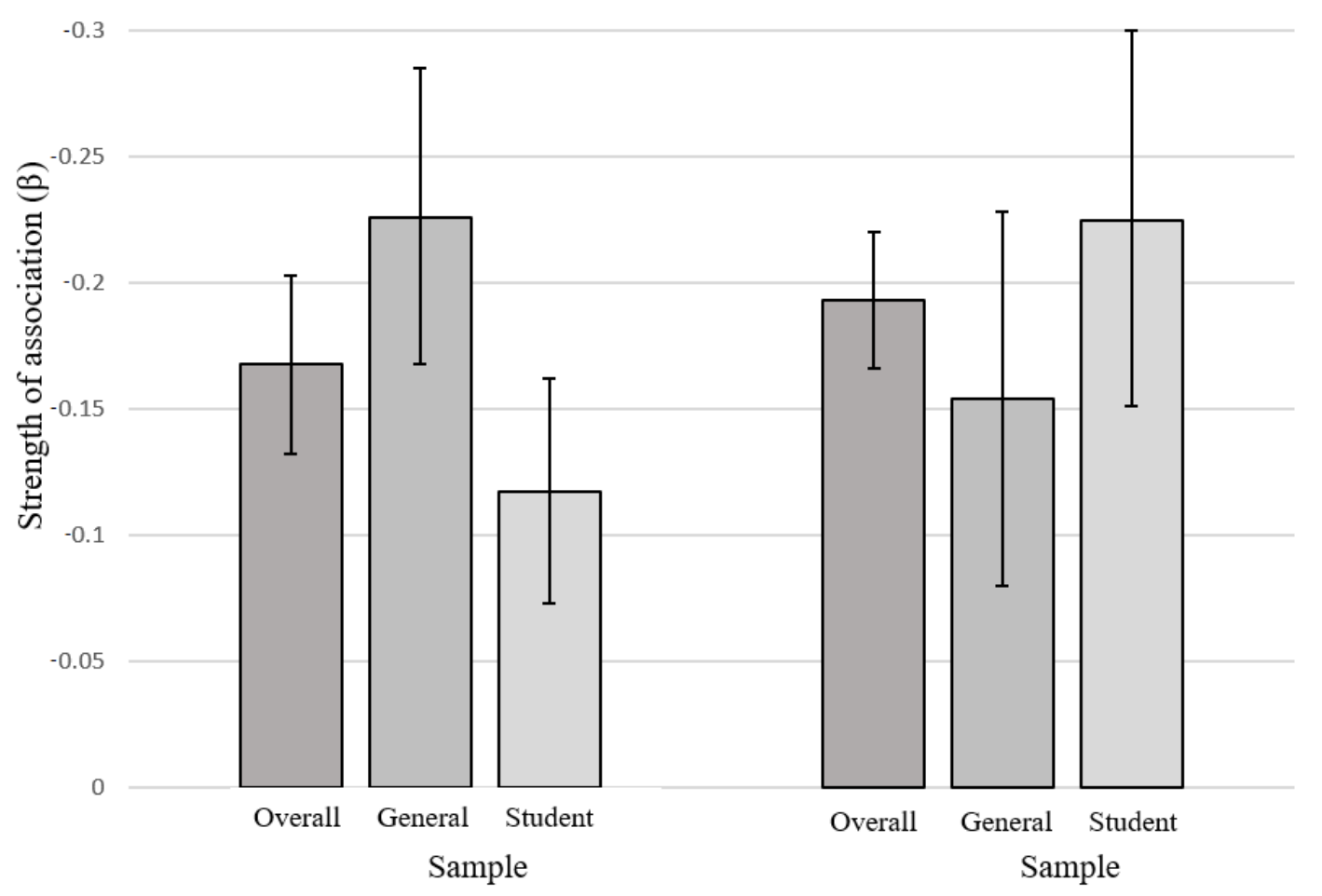

Figure 2.6. Results of meta-analyses between social dominance orientation (left) and right-wing authoritarianism (right), using different sample types and measurement scales.

Consistent with the results of Study $1 \mathrm{~A}$ and H2, the relationship between SDO and environmentalism was again moderated by sample type $(\mathrm{Q}(1)=18.09, p<.001)$, with a stronger association in general population samples $(r=-.226,95 \%$ CI [-.253, $.199], p<.001)$ than in student samples $(r=-.117,95 \%$ CI [-.159, -.075], $p<.001)$. In line with Study 1A, this was not the case for RWA $(\mathrm{Q}(1)=1.87, p=.172)$.

\section{Separate meta-analyses by type of environmental variable}

I then conducted a series of meta-analyses of the different types of environmentalism identified and coded in Study 1A: Climate change belief, support for action, Green politics, and pro-environmental attitudes. I did not compute separate meta-analyses for the environmentalist threat and climate change anxiety aspects of environmentalism, as the data that I had access to did not include these variables. 
Again, I included the standardized regression coefficients for SDO, RWA, and environment-relevant variables when both ideological variable is included as the predictor, to control for their effects, and averaged beta weights when more than one relationship fit into the same category of environment-relevant variable. These results are presented in Table 2.3 and Figure 2.7.

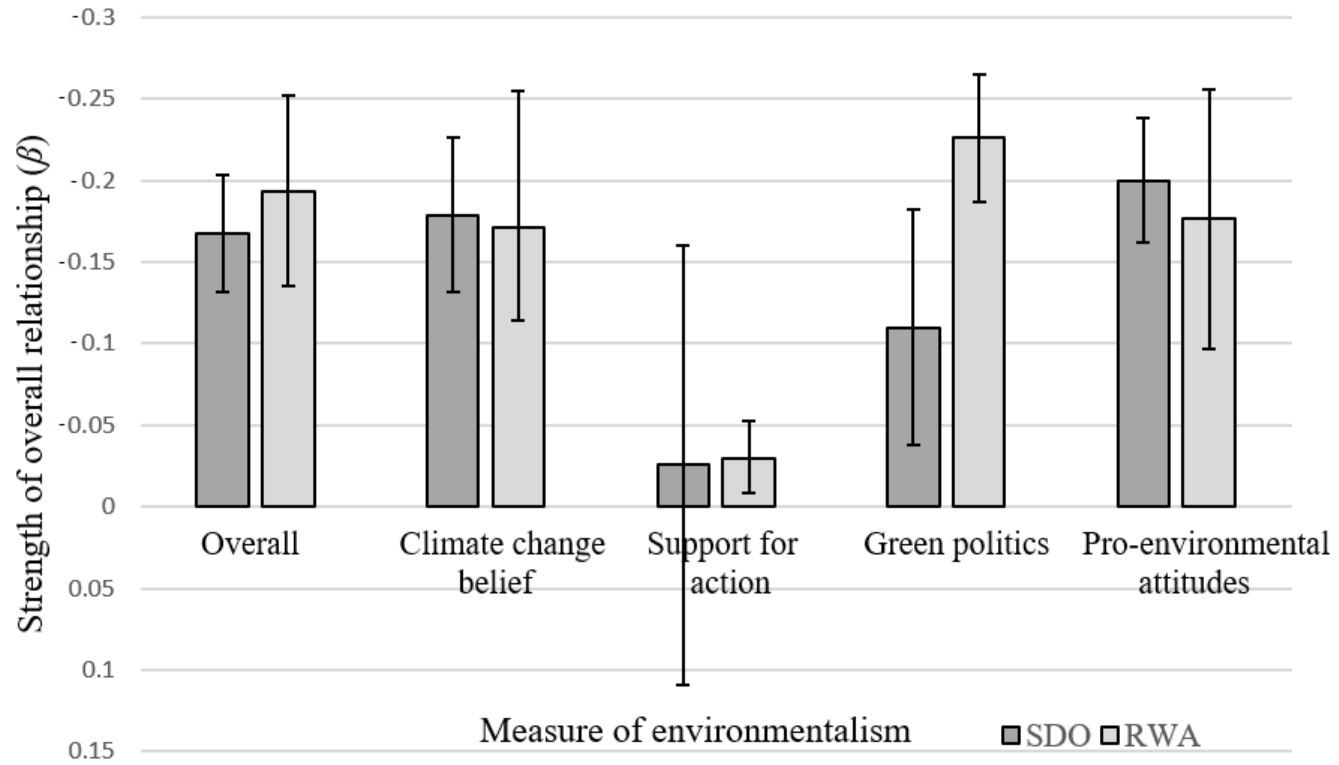

Figure 2.7. Meta-analysed regression coefficients on the associations between SDO (social dominance orientation), RWA (right-wing authoritarianism), and several indices of environmentalism. 
Table 2.3.

Results of the meta-analyses of regression coefficients between SDO, RWA, and four environment-relevant variables.

\begin{tabular}{|c|c|c|c|c|c|}
\hline \multirow[b]{2}{*}{$\begin{array}{l}\text { Measure of } \\
\text { environmentalism }\end{array}$} & \multirow[b]{2}{*}{$\underline{\mathrm{k}}$} & \multicolumn{2}{|c|}{ Social dominance orientation } & \multicolumn{2}{|c|}{ Right-wing authoritarianism } \\
\hline & & $\frac{\text { Overall effect size }}{[95 \% \mathrm{CI}]}$ & $\underline{\text { Variability }}$ & $\frac{\text { Overall effect size }}{[95 \% \mathrm{CI}]}$ & Variability \\
\hline Overall & 16 & $\begin{array}{c}-.17, p<.001 \\
{[-.20,-.13]}\end{array}$ & $\begin{array}{c}\mathrm{Q}(15)=46.83 \\
\mathrm{I}^{2}=67.97\end{array}$ & $\begin{array}{c}-.19, p<.001 \\
{[-.25,-.14]}\end{array}$ & $\begin{array}{c}Q(15)=162.37 \\
I^{2}=90.76\end{array}$ \\
\hline Climate change belief & 9 & $\begin{array}{c}-.18, p<.001 \\
{[-.23,-.13]}\end{array}$ & $\begin{array}{c}\mathrm{Q}(8)=52.93 \\
\mathrm{I}^{2}=84.89\end{array}$ & $\begin{array}{c}-.17, p<.001 \\
{[-.26,-.11]}\end{array}$ & $\begin{array}{c}\mathrm{Q}(8)=171.10 \\
\mathrm{I}^{2}=95.32\end{array}$ \\
\hline Support for action & 6 & $\begin{array}{c}-.03, p=.71 \\
{[-.16, .11]}\end{array}$ & $\begin{array}{c}\mathrm{Q}(5)=49.27 \\
\mathrm{I}^{2}=89.85\end{array}$ & $\begin{array}{c}-.03, p=.008 \\
{[-.05,-.01]}\end{array}$ & $\begin{array}{c}\mathrm{Q}(5)=3.24 \\
\mathrm{I}^{2}=0.00\end{array}$ \\
\hline Green politics & 12 & $\begin{array}{c}-.11, p=.003 \\
{[-.18,-.04]}\end{array}$ & $\begin{array}{c}\mathrm{Q}(11)=53.16 \\
\mathrm{I}^{2}=79.31\end{array}$ & $\begin{array}{c}-.23, p<.001 \\
{[-.27,-.19]}\end{array}$ & $\begin{array}{c}\mathrm{Q}(11)=14.96 \\
\mathrm{I}^{2}=26.49\end{array}$ \\
\hline Pro-environmental attitudes & 11 & $\begin{array}{c}-.20, p<.001 \\
{[-.24,-.16]}\end{array}$ & $\begin{array}{c}Q(10)=26.75 \\
I^{2}=62.61\end{array}$ & $\begin{array}{c}-.18, p<.001 \\
{[-.26,-.10]}\end{array}$ & $\begin{array}{c}Q(10)=164.89 \\
I^{2}=93.94\end{array}$ \\
\hline
\end{tabular}

Note. All Q statistics are significant at the $p<.001$ level. 
These results demonstrate that RWA has a significantly stronger relationship with Green politics when SDO is controlled for, whilst the regression weight with SDO is smaller than the mean correlation reported in Study 1A. I take this to mean that RWA more strongly underlies rejection of Green political parties and policies than the preference for inequality. For the other environment-relevant measures, SDO and RWA have roughly equivalent relationships, with SDO tending to relate slightly stronger to climate change belief and pro-environmental attitudes (though not significantly so).

Both SDO and RWA are significant predictors of all aspects of environmentalism except when this is indexed as support for action: where RWA is a significant predictor, and SDO is not. As well as having the fewest studies including measures of support for action, this category of environmentalism was only included in student samples, which again could explain why findings diverge from the correlations in Study 1A (where SDO and RWA are significant correlates, and these findings come from a mix of general population and student samples).

\section{Variance explained}

To determine how meaningful these associations are, I then examined how much of the variance in environmentalism SDO and RWA explain. This information is included in the SPSS output for the regression analyses, captured by the R-squared value. The R-square indexes the proportion of variance in environmentalism (the dependent variable) that is explained by SDO and RWA (the independent variables in the model). Because it is a proportion, when multiplied by 100 it gives the percentage of the variance in the dependent variable explained by the predictors. I have more than one predictor in each model, which can cause the R-square to increase due to chance, therefore I present the adjusted R-squared value (which controls for the number of predictors in the model, so that additional variance explained by chance is controlled for). Using this value, I can compute the average amount of variance the models explain in environmentalism overall, as well as the four different types of environmentalism included in this study.

For environmentalism overall, the mean adjusted R-square value is .083 ( $S D=$ .076). This is interpreted as SDO and RWA explaining $8.3 \%$ of the variance in environmentalism. The value is skewed, with more low R-square values and fewer 
higher values, meaning that for most models, a relatively small amount of the variance is explained. This is likely in part because the models do poorly at predicting support for action, explaining on average just two percent of the variance. The variables explain a more substantial portion of variance in climate change belief (on average $6.8 \%$ ), and are relatively better at predicting support for Green politics $(10.5 \%)$ and pro-environmental attitudes $(11.4 \%)$.

\section{Comments}

I conducted Study 1B as I was interested in pinpointing the unique associations between SDO, RWA and environmentalism. I show that controlling for each ideological variable decreases the others' association with environment-relevant variables (i.e. compare the average beta weights reported in Table 1 here to the mean correlations from Study 1A). This indicates that for each relationship with SDO, the correlation captures part of the association driven by RWA and its shared variance with SDO, and the same for the RWA-environmentalism links. Examining the unique association gives a more accurate estimate of the unique association between each ideological variable and environmentalism. In doing this, I found that each ideology explains a unique proportion of the variance in environmentalism, but again they are roughly equivalent predictors. The one exception to this is for Green politics, where RWA is a stronger predictor than SDO.

Replicating the finding from Study 1A, the regression coefficient with environmentalism overall was notably stronger in general population samples than student samples for SDO. This is consistent with my prediction and findings from Study 1 A.

The aim of Study 1B was to evaluate the unique effects of SDO and RWA on environment-relevant variables. Much like Study 1A, these analyses showed that the ideological variables have comparable associations. I turn now to a discussion of all meta-analyses reported in Studies 1A and 1B. 


\section{Discussion of Studies 1A and 1B}

In Study 1A, I showed that both SDO and RWA are important correlates of a range of environment-relevant variables, with an especially strong relationship for SDO in general population samples, and for both SDO and RWA when environmentalism is operationalized as fear of action on climate change or environmentalists themselves. Building on this work, Study 1B meta-analyzed the unique contributions of SDO and RWA in predicting environmentalism. While I expected findings of both studies to converge to show that SDO is the stronger ideological correlate and predictor of environment-relevant variables, Study 1B controlled for the effect of SDO to show that overall, RWA is a slightly stronger predictor.

Together, Studies 1A and 1B, drawing from findings of over thirty-thousand and twenty-thousand participants (respectively), demonstrate the importance of ideology for understanding human-environment relations. Meta-analysis is a useful tool for combining findings from similar but independent studies to better estimate a true effect size: in this case, a relationship between variables (Erez et al., 1996). My meta-analyses show that when combined, findings converge to show that SDO and RWA have important associations with environmentalism, both together and uniquely. We can therefore be more confident that this relationship exists, and conclude that researchers should consider the impact of these ideological variables for our understanding of the potential barriers to belief and action on climate change.

Information about climate change is selectively sought after, remembered, and used in ways that are consistent with people's ideological stances (Hornsey et al., 2016). For those endorsing authoritarian, hierarchical or dominance-based attitudes, this is more likely to mean rejecting climate change and opposing action on environmental issues. This represents a more significant barrier to motivating mitigating action on climate change than simply a lack of knowledge, thus helping to explain why educating people on such issues is not sufficient to spur action.

This indicates that climate change communications ought to be careful in how they portray the issue and solutions. Hakkinen and Akrami (2014) suggest avoiding framing action on climate change as threatening to the social hierarchy, as this might make Social Dominants especially unlikely to act. My findings suggest that 
Authoritarians may also need tailored communication strategies, although it is as yet unclear why they endorse anti-environmental attitudes (e.g. Schultz \& Stone, 1994 versus Reese, 2012).

Both sets of meta-analyses show that ideology relates to a variety of environment-relevant variables: Social Dominants and Authoritarians are not just sceptics on climate change, but they also oppose action or policies aimed at addressing the problem, feel less anxiety about climate change, instead feeling that the threat comes from environmental activists. Duckitt (2001) wrote about the generality of prejudice, where people's prejudiced attitudes towards one out-group tend to extend to other out-groups. Similar findings led Adorno et al. (1950) to argue that some people hold a general attitudinal syndrome made up of their in-group favouritism, out-group prejudice, profascist attitudes, and politicoeconomic conservatism. Through extending studies on the link between ideology and prejudice into the environmental domain, it appears that the anti-environmentalism associated with SDO and RWA is similarly generalizable to multiple aspects of environmentalism. With SDO and RWA explaining about half of the variance in prejudice, the ideologies are certainly less strongly related to environmentalism, but still make a sizeable contribution.

In the social sciences, small effect sizes are not uncommon, and are in part a reflection of the complexity of the phenomena we study. There are many factors that are relevant for predicting pro-environmentalism (e.g. see Chapter 1 for a brief overview), and even small contributions to behaviours may translate to meaningful differences in the environmental consequences of ones choices. To put these effect sizes into context, the most recent estimates suggest that the average New Zealander expends around 7.69 metric tonnes of carbon dioxide per year (Worldbank, 2014), which is among the highest in the world. Greenhouse gas emissions in New Zealand have grown by close to $25 \%$ since 1990 , and the country has pledged to reduce its total emissions by 50\% below 1990 levels by 2050 (New Zealand Gazette, 2011). This target will require individuals to do their part to reduce their own contributions, and the practical significance of even weak determinants of behaviour should not be overlooked. My research shows that ideological attitudes explain around one tenth of the variance in environmentalism. These attitudes are also relatively stable over time and perhaps more difficult to shift than many of the factors explaining the remainder 
of the variance in environmentalism. This further highlights the importance of researching the nature of this ideology-environmentalism link.

In the research synthesized to date, all associations between SDO, RWA and environmentalism have been cross-sectional in nature. These findings are easily combined using meta-analysis, and can tell us a great deal about the nature of the association between ideology and environmentalism: They are indeed related, under almost all conditions, yet there are some differences in the strengths of the associations that depend on the sample and aspect of environmentalism captured. However, longitudinal examination of these relations is needed to be able to more fully grasp the nature of the associations.

From the results of my first two studies, I conclude that people's current levels of endorsement of SDO and RWA tend to relate to lower levels of environmentalism within the same measurement point, yet this relationship may not extend to predict their attitudes in future. Longitudinal data follows participants across time, thereby picking up any directional relationships between variables. No research to date has examined the longitudinal associations between SDO, RWA and environmentalism. In the next chapter, I present two studies that aim to address this gap in the literature. 


\section{Chapter 3}

\section{Examining the associations between ideology and environmentalism over time}

\section{Introduction to Study 2A and 2B}

The studies in Chapter 2 establish SDO and RWA as important and independent correlates of environmentalism. However, Studies 1A and 1B, as well as the broader literature on the ideological determinants of environmentalism, have yet to explore whether and how these ideological attitudes relate to environment-relevant variables over time.

It is possible that the ideological and environmental variables covary only within time points (i.e., do not exhibit a longitudinal association). Those who favour inequality or tradition and loyal submission to authority may express negative attitudes towards the environment, but independent of ideology. Alternatively, one's ideology could drive their later climate change beliefs and attitudes on environmental action. Another option is that environmental attitudes drive ideological positions. Finally, ideological and environmental attitudes could be reciprocally related. The aim of the studies presented in Chapter 3 is to test these possibilities.

One reason to believe that endorsement of SDO and RWA would precede environmental attitudes follows the logic of Duckitt's (2001) dual-process model. Duckitt asserts that prior levels of endorsement of SDO and RWA work to predict attitudes towards different social groups in a causal manner: people prefer their ingroup, and are hostile towards those unlike themselves because they view the world as competitive and thus have a desire to hold a superior position over other groups (i.e., SDO). Similarly, for RWA, individuals might view the world as threatening and thus rely on submitting to authorities and traditional norms and punishing those who transgress (RWA), hence favoring their ingroup. Both SDO and RWA have been applied to the environmental domain, evidencing modest correlations between each 
ideological variable and environment-relevant variables (e.g. see Chapter 2). A causal relationship between SDO, RWA and environmentalism would therefore be consistent with theoretical predictions about the relationship between ideology and attitudes over time (Duckitt, 2001), and strengthen their utility as ideological predictors of environmentalism.

Although not directly tested, some research on these associations makes causal claims about how the variables are related. For example, Pratto et al. (1994) reported an association between SDO and opposition to environmental policies, concluding that Social Dominants oppose policy that promotes equality between humans and other species. Milfont et al. (2013) make a similar claim, arguing that the link between SDO and environmental exploitation is due to their support for human dominance over nature. Milfont and Sibley (2014) proposed an opposing viewpoint where the exploitation comes instead from high-SDO's attempt to widen the gap between high and low status groups through unequal distribution of natural resources. Arguing the SDO-environmentalism link exists because of either of these explanations suggests that it is people's ideology that is driving their environmental attitudes, hence a causal argument.

Similarly, investigators of the relationship between RWA and environmentalism have suggested that the preference for tradition (Schultz \& Stone, 1994) or for both tradition and aggression towards those who reject authority (Reese, 2012) underlie the association. Despite this, proponents of these perspectives have not empirically tested the causal nature of these relationships longitudinally.

The studies in this chapter assess the interrelationships between SDO, RWA, and environment-relevant variables over time. First, in Study 2A I explore these associations within a student population sampled twice over five months. To replicate and extend on this work, in Study 2B I draw on data from a national probability sample surveyed annually over five years. 


\section{Study 2A}

\section{Exploring short-term longitudinal effects of social dominance orientation and right-wing authoritarianism on environmentalism ${ }^{4}$}

\section{Introduction}

Longitudinal research helps to establish temporal connections between variables, determining whether one variable precedes the other, if there is a bidirectional association over time, or perhaps no relation at all. In Study 2A, I present the first study on the effects of SDO and RWA on environment-relevant variables over time, using a student sample. Environmentalism was indexed as general pro-environmental attitudes, as well as a specific measure of belief in anthropogenic climate change.

I make several predictions for this study. Firstly, I expect to find that each variable is relatively stable over time (i.e. strong association between time 1 and time 2 scores). This is because research on the test-retest reliability of ideological and attitudinal variables suggests they remain largely unchanged over time (e.g. Liu, Huang \& McFedries, 2008; Sidanius et al., 2013; H1).

On the association between SDO and RWA, Duckitt et al. (2002) argue this depends on context. The strength of the relationship between SDO and RWA is stronger in countries with more ideologically organized politics, unrelated where the politics of a society is not ideologically organized, and directional (i.e. one more strongly relating to changes in the other) where individuals are socialised more strongly into one ideology than the other. Duckitt (2001) argues that politics in New

${ }^{4}$ Note. Study $2 \mathrm{~A}$ has been rewritten for inclusion in the thesis from the publication:

Stanley, S. K., Wilson, M. S., \& Milfont, T. L. (2017). Exploring short-term longitudinal effects of social dominance orientation and right-wing authoritarianism on environmentalism. Personality and Individual Differences, 108, 174-177. 
Zealand are relatively strongly tied to ideology, and I therefore expect that both variables will work to reinforce the other over time, hence exhibiting a bidirectional association (H2).

I further hypothesize, based on theory (e.g., Duckitt, 2001) and some empirical evidence (e.g., Duckitt \& Sibley, 2010; Duckitt et al., 2002) showing SDO and RWA to precede attitudes towards various social groups, that SDO and RWA will be significant prospective predictors when extended to predict environmentalism over time $(\mathrm{H} 3)$. However, informed by the findings of Chapter 2, I expect the variables will have associations with environmentalism that are equivalent in strength (H4).

\section{Method}

\section{Participants and procedure}

These data comprise responses from first-year psychology students invited to fill in an online questionnaire at the start of each semester towards a mandatory course research requirement. Each student participated twice, with approximately five months in between measurement points. The survey was made available in both 2014 and 2015, thus two cohorts of students participated and data were combined to make up the final dataset, which includes data from 674 students $(77 \%$ female; mean age $=$ 18.7 years).

\section{Measures}

I was interested in participant's scores on SDO, RWA, and environmentrelevant variables, though participants completed several small 'packets' of measures pertaining to a variety of short research studies. This is common practice for largescale research projects, and no manipulations were introduced prior to exposure to the measures included in this study that could have affected participant responses. For my variables of interest, participants were asked to respond on 7-point Likert scales from 1 (strongly disagree) to 7 (strongly agree). Table 3.1 includes alpha coefficients for the reliability of each measure, which are all acceptable (i.e., $\alpha$ 's $<.70$; Nunnally, 1978). Each balanced scale included a range of pro-trait and con-trait items, where the con-trait items were recoded prior to scale calculation and analysis. 
Social dominance orientation. This study employed Ho et al.'s (2012) $\mathrm{SDO}_{7}$ scale to measure endorsement of SDO. This measure includes a total of 16 items, with eight items to measure the 'anti-egalitarian' dimension of SDO (including four protrait items, e.g. "We shouldn't try to guarantee that every group has the same quality of life", and four con-trait items, e.g. "We should do what we can to equalize conditions for different groups") and eight balanced items to measure SDOdominance (e.g. "Some groups of people are simply inferior to other groups"; "Groups at the bottom are just as deserving as groups at the top").

Right-wing authoritarianism. Endorsement of RWA was measured using Altemeyer's (1996) RWA scale. This scale contains 30 balanced items, with a range of pro-trait (e.g. "It is always better to trust the judgment of the proper authorities in government and religion than to listen to the noisy rabble-rousers in our society who are trying to create doubt in people's minds") and con-trait (e.g. "Atheists and others who have rebelled against the established religions are no doubt every bit as good and virtuous as those who attend church regularly") items.

Environmentalism. I used two measures of environment-relevant attitudes, to measure both general pro-environmental attitudes, and specific beliefs about climate change. Dunlap and Van Liere's (1978) New Environmental Paradigm (NEP) Scale was used to measure pro-environmental attitudes. Endorsement of the NEP indicates a preference for harmonious relations between humans and nature. The NEP is a widely used measure of environmental attitudes (Dunlap, 2008), with studies consistently reaffirming its reliability (e.g. Cordano, Welcomer \& Cherer, 2003; Hawcroft \& Milfont, 2010). The scale is comprised of 12 items, including an equal number of protrait ("Humans must live in harmony with nature in order to survive") and con-trait ("Humans have the right to modify the natural environment to suit their needs") items. Finally, climate change belief was measured using two related items $(r=.70$, $95 \%$ CI $[.65, .74])$, “Climate change is an entirely natural phenomenon - human action does not contribute importantly to it" and "Any changes in global climate simply reflects naturally occurring variation" that were combined to form a two-item scale. 


\section{Data analysis}

I used SPSS (IBM, version 23) to calculate mean scores for each scale, and then computed descriptive statistics, including means and correlations, for each of the variables. To answer my main research question, about the temporal associations between SDO, RWA and environmentalism, I constructed a latent cross-lagged panel model in Mplus 7.3 (Muthén \& Muthén, 1998-2010). Mplus is a statistical modeling program that allows users to run structural equation models using syntax.

I wrote the Mplus syntax to estimate SDO, RWA and environmentalism using latent variables. Many measures in psychology are said to tap 'hypothetical constructs' (Harman, 1960), which might not refer to something that exists objectively in the real world, but instead something that researchers invent (Nunnally, 1978). Bollen (2002) uses the example of self-esteem, which does not exist in an objective sense, but researchers can think of, define, and use scales to estimate it. Our variables of interest, SDO, RWA, and environmental attitudes, are similarly hypothetical constructs, and perhaps as with most latent variables, they "cannot be directly measured” (Jöreskog \& Sörbom, 1979, p. 105). Researchers estimate participants 'levels' on these constructs based on how they respond to multiple items, thus improving the reliability of the measurement (Diamantopoulos, Sarstedt, Fuchs, Wilczynski \& Kaiser, 2012).

The individual items making up the latent variable vary and covary because responses on the items are driven by the underlying (unmeasured) latent variable, thus the latent variable has been estimated indirectly through agreement with observed variables (i.e. the items making up each scale). To take into account the variance and covariance between observed variables, I use latent variables rather than mean scores to estimate each construct in the model.

Therefore, the first step in constructing the model was to create latent variables for SDO, RWA, scores on the NEP, and climate change belief for both Time 1 and Time 2. Next, I allowed each item to covary between Time 1 and Time 2, and allowed the factors within each time point to correlate. To test the model, I set each Time 1 latent variable to predict each Time 2 latent variable. When the same latent variable predicts itself, e.g. from SDO at Time 1 to SDO at Time 2, this residualizes 
the Time 2 variable to now measure change in SDO over time. The $\mathrm{R}^{2}$ statistic gives a measure of the amount of variance explained in each dependent variable by the predictor variables.

I relied on several fit indices to evaluate the model. The Chi-Square test indicates good fit when the value is not significant and relatively small compared to its degrees of freedom. This test is sensitive to model complexity and sample size (Potthast, 1993; Yung \& Bentler, 1994), with the test virtually always returning a significant result when sample size is above 400 (Kenny, 2015). Dividing the ChiSquare value by its degrees of freedom is one proposed correction for this limitation (Wheaton, Muthen, Alwin \& Summers, 1977), however is similarly flawed, as the guidelines on what constitutes an acceptable value are unclear (Brown, 2014). I tentatively evaluate the $\chi^{2} / \mathrm{df}$ as indicating good fit when the value is below 3 , as per Carmines and McIver's (1981) suggestion, though I do so in conjunction with several other fit indices to make up for the shortcomings of this test.

Further indicative of good fit, the comparative fit index (CFI) should be above .95 , though this index is also affected by model complexity (Hu \& Bentler, 1999; Kenny, 2015). The standardized root mean square residual (SRMR) should be below .08 (with 0 indicative of perfect fit; $\mathrm{Hu} \&$ Bentler, 1999). Finally, an RMSEA of .01 indicates excellent fit, .05 good, and .08 mediocre (MacCallum, Browne \& Sugawara, 1996). Where structural equation models are presented throughout my thesis (e.g. Study 2B, Studies 3A-3C), I rely on these same indicators to assess fit unless otherwise stated.

\section{Results}

\section{Descriptive statistics}

As displayed in Table 3.1, mean endorsement of SDO, RWA, and climate change denial was low, whereas overall scores on the NEP were relatively high (for 7point scales). Furthermore, zero-order correlations show that SDO and RWA are related to both facets of environmentalism: those endorsing SDO and RWA tend to score higher on climate change denial and report lower pro-environmental attitudes. While the strengths of these associations are comparable, RWA is notably more strongly related to scores on the NEP than SDO in Time 2 only. This is consistent 
with findings from Study 1A, where Pearson's correlation coefficients with environment-relevant variables are comparable for both SDO and RWA. The crosslagged panel model reports standardized beta weights, which control for each variable to test the comparative strengths of the associations.

\section{Cross-lagged panel model findings}

Model fit. The model, illustrated in Figure 3.1, showed acceptable fit to the data. Although the Chi-Square test returned a significant result $\left(\chi^{2}(6932)=16172, p\right.$ $<.001)$, meaning that there was a significant difference between my model and a hypothetical 'ideal' model, dividing the Chi-Square value by its degrees of freedom returned a satisfactory fit $\left(\chi^{2} / \mathrm{df}=2.33\right)$. Furthermore, the CFI, which is similarly sensitive to model complexity, was slightly below the threshold of good fit (.76), whereas the sRMR (.07) and RMSEA $(.04,90 \%$ CI $[.04, .05])$ both indicated good fit. Taken together, and given the limitations of the two measures demonstrating only marginally acceptable fit, I accepted the model.

The model also succeeds at explaining the variance in Time 2 variables: $57.4 \%$ of the variance in SDO, $63.4 \%$ in RWA, $46.7 \%$ in climate change denial, and $59.7 \%$ in pro-environmental attitudes (as calculated from the $\mathrm{R}^{2}$ statistic). 
Table 3.1.

Descriptive statistics, and correlation matrix between mean scores of each variable.

\begin{tabular}{|c|c|c|c|c|c|c|c|c|c|c|}
\hline & Mean & SD & 1. & 2. & 3. & 4. & 5. & 6. & 7. & 8. \\
\hline 1. SDO T1 & $\begin{array}{c}2.82 \\
{[2.75,2.89]}\end{array}$ & $\begin{array}{c}.92 \\
{[.88, .97]}\end{array}$ & .88 & & & & & & & \\
\hline $\begin{array}{l}\text { 2. RWA } \\
\text { T1 }\end{array}$ & $\begin{array}{c}2.70 \\
{[2.64,2.75]}\end{array}$ & $\begin{array}{c}.76 \\
{[.73, .80]}\end{array}$ & $\begin{array}{c}.54 \\
{[.48, .59]}\end{array}$ & .91 & & & & & & \\
\hline 3. NEP T1 & $\begin{array}{c}5.22 \\
{[5.16,5.28]}\end{array}$ & $\begin{array}{c}.76 \\
{[.72, .79]}\end{array}$ & $\begin{array}{c}-.30 \\
{[-.38,-.23]}\end{array}$ & $\begin{array}{c}-.41 \\
{[-.47,-.35]}\end{array}$ & .78 & & & & & \\
\hline $\begin{array}{l}\text { 4. Denial } \\
\text { T1 }\end{array}$ & $\begin{array}{c}2.84 \\
{[2.84,3.06]}\end{array}$ & $\begin{array}{r}1.40 \\
{[1.33,} \\
1.47]\end{array}$ & $\begin{array}{c}.29 \\
{[.22, .37]}\end{array}$ & $\begin{array}{c}.26 \\
{[.19, .34]}\end{array}$ & $\begin{array}{c}-.34 \\
{[-.42,-.26]}\end{array}$ & .78 & & & & \\
\hline 5. SDO T2 & $\begin{array}{c}2.92 \\
{[2.84,3.00]}\end{array}$ & $\begin{array}{c}.99 \\
{[.95,1.03]}\end{array}$ & $\begin{array}{c}.71 \\
{[.67, .75]}\end{array}$ & $\begin{array}{c}.51 \\
{[.45, .57]}\end{array}$ & $\begin{array}{c}-.28 \\
{[-.35,-.21]}\end{array}$ & $\begin{array}{c}.30 \\
{[.22, .37]}\end{array}$ & .91 & & & \\
\hline $\begin{array}{l}\text { 6. RWA } \\
\text { T2 }\end{array}$ & $\begin{array}{c}2.74 \\
{[2.67,2.81]}\end{array}$ & $\begin{array}{c}.88 \\
{[.84, .91]}\end{array}$ & $\begin{array}{c}.50 \\
{[.45, .56]}\end{array}$ & $\begin{array}{c}.76 \\
{[.71, .80]}\end{array}$ & $\begin{array}{c}-.36 \\
{[-.43,-.30]}\end{array}$ & $\begin{array}{c}.26 \\
{[.19, .33]}\end{array}$ & $\begin{array}{c}.64 \\
{[.59, .69]}\end{array}$ & .94 & & \\
\hline 7. NEP T2 & $\begin{array}{c}5.21 \\
{[5.15,5.28]}\end{array}$ & $\begin{array}{c}.87 \\
{[.83, .90]}\end{array}$ & $\begin{array}{c}-.30 \\
{[-.38,-.23]}\end{array}$ & $\begin{array}{c}-.41 \\
{[-.47,-.33]}\end{array}$ & $\begin{array}{c}.70 \\
{[.65, .74]}\end{array}$ & $\begin{array}{c}-.45 \\
{[-.51,-.37]}\end{array}$ & $\begin{array}{c}-.40 \\
{[-.47,-.33]}\end{array}$ & $\begin{array}{c}-.53 \\
{[-.59,-.47]}\end{array}$ & .85 & \\
\hline $\begin{array}{l}\text { 8. Denial } \\
\text { T2 }\end{array}$ & $\begin{array}{c}2.84 \\
{[2.73,2.95]}\end{array}$ & $\begin{array}{r}1.37 \\
{[1.31} \\
1.43] \\
\end{array}$ & $\begin{array}{c}.23 \\
{[.15, .30]}\end{array}$ & $\begin{array}{c}.28 \\
{[.21, .35]}\end{array}$ & $\begin{array}{c}-.33 \\
{[-.41,-.26]}\end{array}$ & $\begin{array}{c}.55 \\
{[.49, .61]}\end{array}$ & $\begin{array}{c}.38 \\
{[.31, .45]}\end{array}$ & $\begin{array}{c}.39 \\
{[.33, .46]}\end{array}$ & $\begin{array}{c}-.45 \\
{[-.51,-.37]} \\
\end{array}$ & .82 \\
\hline
\end{tabular}

Note. $\mathrm{SDO}=$ social dominance orientation, $\mathrm{RWA}=$ right-wing authoritarianism, $\mathrm{NEP}=$ new environmental paradigm, $\mathrm{T} 1=\mathrm{Time} 1, \mathrm{~T} 2=\mathrm{Time}$

2. All Pearson's correlations are statistically significant at $p<.001$. Numbers in square brackets are $95 \%$ confidence intervals based on bootstrapping of 1000 iterations. Along the diagonal are Cronbach's alphas for each scale. 


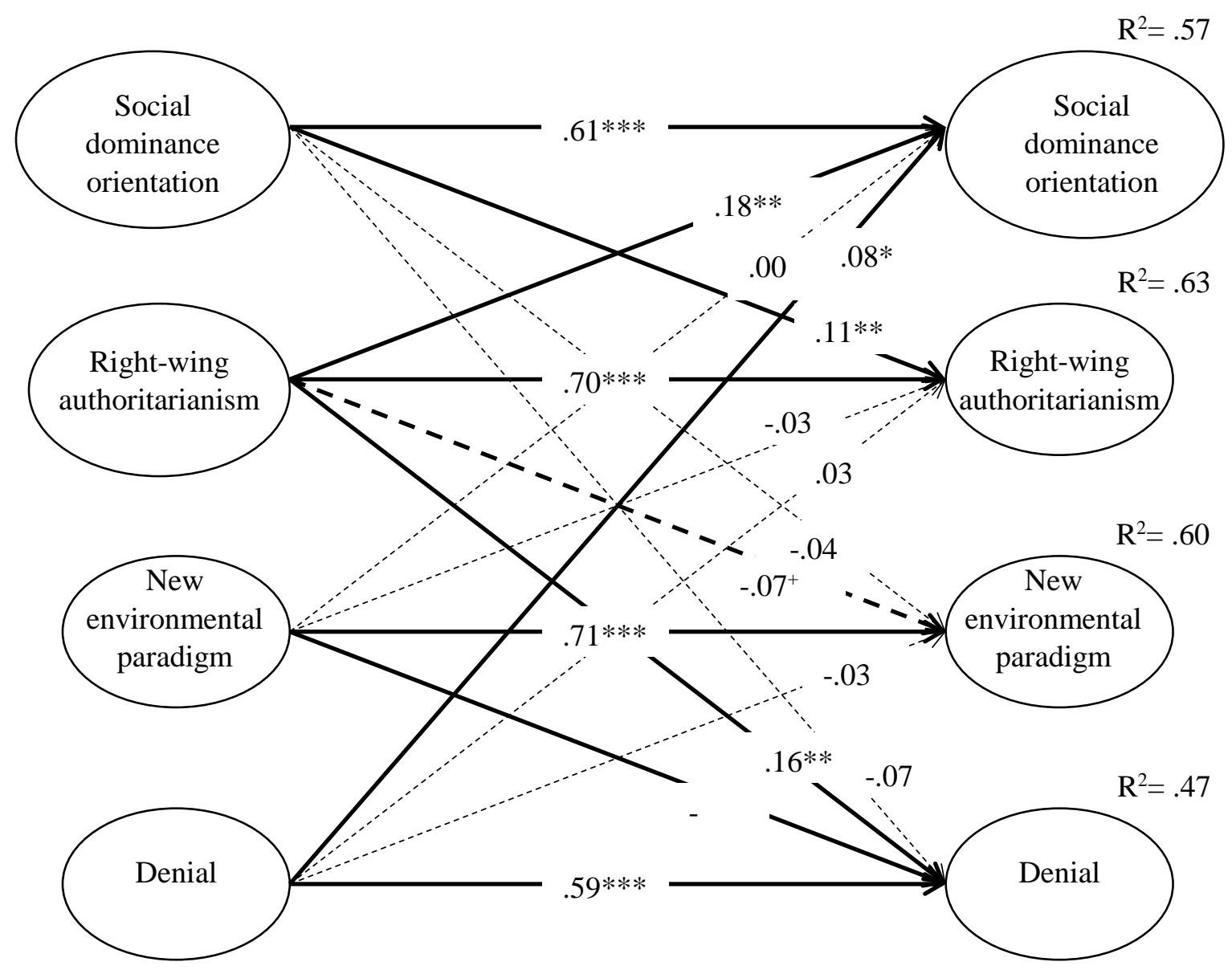

Figure 3.1. Standardized associations between SDO, RWA, NEP, and climate change denial across time.

Note. ${ }^{*} p<.05, * * p<.01, * * * p<.001,{ }^{+}$marginally significant, non-significant paths dashed.

Stability over time. Each variable exhibited a strong and significant association between initial and final measurement points $\left(\beta_{\mathrm{SDO}}=.61,95 \% \mathrm{CI}[.54\right.$, $.67] ; \beta_{\mathrm{RWA}}=.70,95 \% \mathrm{CI}[.64, .76] ; \beta_{\mathrm{NEP}}=.71,95 \% \mathrm{CI}[.65, .78] ; \beta_{\text {Denial }}=.59,95 \%$ CI $[.51, .67])$. This indicates that they are relatively stable over time, and in other words have high test-retest reliability, thus supporting H1. Because of their strengths, these autoregressive paths are explaining the majority of the variance in the Time 2 variables. For example, the standardized coefficient from Time 1 endorsement of NEP to endorsement at Time 2 suggests that approximately 50\% of Time 2 NEP scores is explained by the association with Time 1 NEP scores. The cross-lagged paths are 
therefore only able to explain variation in the Time 2 NEP scores beyond that accounted for by NEP itself at Time 1 .

Relationship between SDO and RWA over time. Turning to the crosslagged effects, Figure 1 shows that RWA and SDO exhibit a bidirectional association. RWA has a positive influence on SDO over time $\left(\beta_{\mathrm{RWA}}=.18, p<.001,95 \%\right.$ CI [.10, .26]), and a similar, albeit weaker effect exists between initial levels of SDO and later increased endorsement of RWA $\left(\beta_{\mathrm{SDO}}=.11, p=.002,95 \%\right.$ CI $\left.[.04, .18]\right)$. Therefore, the ideologies positively reinforce each other over time.

\section{Relationship between environmental variables over time. While NEP} prospectively predicts a reduction in climate change denial $(\beta=-.10, p=.027,95 \%$ CI [-.20, -.01]), this effect is unidirectional, with no longitudinal relationship from initial levels of denial to later endorsement of the NEP $(\beta=-.03, p=.497,95 \%$ CI [$.10, .05])$. Therefore, while pro-environmental attitudes are related to an increase in climate change belief over time (effectively, the reduction of climate change denial), initial climate change denial does not influence later endorsement of proenvironmental attitudes. The extent to which this finding points to a causal relationship is explored more fully in the Comments section.

Testing the research question. I expected that both SDO and RWA would be prospective predictors of environmentalism. I found that RWA significantly predicts increases of climate change denial over time $\left(\beta_{\mathrm{RWA}}=.16, p=.001,95 \%\right.$ CI [.06, $.25]$ ), and is only marginally predictive of changes in scores on the NEP over time ( $\beta$ $=-.07, p=.081,95 \% \mathrm{CI}[-.15, .01])$. Unexpectedly, SDO does not predict changes in either the NEP $(\beta$ SDO $=-.04, p=.365,95 \%$ CI $[-.12, .04])$ or denial $(\beta=-.07, p=.155$, $95 \%$ CI $[-.16, .03])$. Therefore, the prediction that both ideologies would exhibit significant longitudinal associations with environmental attitudes was not supported. In the case of climate change denial, I did not find evidence for $\mathrm{H} 4$, that the associations would be equivalent between ideological attitudes, on the basis of the non-overlapping confidence intervals, which indicate that RWA is a significantly stronger predictor. However, the confidence intervals do overlap in the prediction of scores on the NEP, indicating that the ideologies may not significantly differ as predictors. 
Considering the effect of gender. While some research shows that gender is important in the relationship between ideology and environmental attitudes (e.g. Jylhä et al., 2016), other research shows gender has a minimal effect on belief in climate change (Hornsey et al., 2016). In Chapter 2, I synthesized the literature to find that the gender composition of samples did not influence the strengths of the associations between SDO, RWA, and environmentalism. However, this could have been due to lack of power in the analysis, and therefore in this study I include an exploratory analysis as a further test for the effect of gender in this model.

I did this by using multi-level modeling in Mplus with gender as the grouping variable. This returns two models: One where the estimates for male and females are constrained to equality (the null model), and one with free estimates (the alternative model). If the alternative model does not provide a significantly better fit to the data than the null model, this means that the relationships hold equally for men and women, and therefore is interpreted to mean that there are unlikely to be significant differences between males and females. Indeed, I did not find a significant difference in model fit based on the Satorra-Bentler scaled Chi-square difference test $\left(\chi^{2}(16)=\right.$ $15.796, p=.467)$, and thus conclude that the main model holds equally well for men and women.

\section{Comments}

The aim of Study $2 \mathrm{~A}$ was to contribute to the existing literature on the relationship between ideological variables and environmentalism by conducting the first longitudinal study on these associations. As well as demonstrating that all variables remain relatively stable across time, I found a bidirectional association between both SDO and RWA, and a unidirectional association from NEP to climate change denial. While the first relationship has already been established in the literature (e.g. Duckitt et al., 2002), the latter is a novel finding.

I show that individual's preference for humans to live harmoniously with nature (i.e. scores on the NEP scale) is associated with an increase in their certainty of the reality of climate change. However, belief in climate change does not translate into greater pro-environmental attitudes at a later date. This temporal association is 
consistent with Hornsey et al. (2016) treating scores on the NEP as a precursor or antecedent of one's climate change beliefs, rather than a consequence of belief.

The NEP scale is a measure of worldview, providing insight into how an individual believes we ought to interact with the natural environment. For instance, the items emphasize the extent to which people prefer sustainable human-nature relations (e.g. "Humans must live in harmony with nature in order to survive") versus anthropocentric or exploitative interactions (e.g. "Humankind was created to rule over the rest of nature"). These attitudes may be foundational for later belief in climate change because belief was indexed as belief in the human causes of climate change. One might first need to acknowledge our responsibility to live harmoniously with nature before accepting the problems that the alternative (an environmentally exploitative lifestyle) contributes to: climate change.

This finding indicates that promoting harmonious relations with nature (as in the NEP) could boost climate change belief over time, and hence be included in climate change communication strategies. Instead, focusing on increasing climate change belief (which is the strategy of information deficit models) may have little-tono effect on pro-environmental attitudes.

An alternative explanation for the lack of a reciprocal effect of belief on attitudes is that the NEP is more stable than climate change belief. Specifically, the autoregressive path explains around half of the variance in scores on the NEP at Time 2 , whilst climate change denial explains around $35 \%$. This means that there is less variance to explain in endorsement of NEP at Time 2, hence contributing to lower cross-lagged associations. Providing support for this explanation, although Time 1 RWA exhibited a stronger zero-order association with Time 2 NEP than Time 2 climate change denial (i.e. $r$ 's of -.41 and .28 , respectively), the association with Time 2 NEP dropped to a marginal, weak association when Time 1 NEP was controlled. The high stability of NEP scores could contribute to this drop, and similarly explain why denial is not significantly associated with changes in NEP over time.

As demonstrated by their zero-order correlations, there are consistent, negative associations between SDO, RWA and environment-relevant variables, both within and across time points. Those who endorse SDO and RWA tend to also deny climate 
change and have lower scores on pro-environmental attitudes. This replicates and extends on findings of existing research that demonstrate negative cross-sectional associations between these ideological variables and environmentalism (e.g., Häkkinen \& Akrami, 2014; Milfont et al., 2013).

Similar to findings from Chapter 2, I found that controlling for each ideology (as in the structural equation model) weakens their individual associations with environment-relevant variables. This is taken as evidence that some of the relationship between SDO, RWA and environmentalism is shared between the ideological predictors. Furthermore, the model supports RWA as the stronger ideological predictor of environmentalism, whilst SDO is not a significant predictor of either environment-relevant variable when controlling for RWA. This contradicts my hypotheses, as I had expected both ideologies to predict changes in environmentalism over time (H3), at similar rates (H4). Instead, only RWA had significant lagged effects on environmentalism, and in the case of climate change denial, RWA has a statistically stronger relationship when compared to SDO.

To explore the reasons why SDO might be unrelated to changes in environmentalism across time, I return to the key findings of the moderation analyses in Chapter 2. Both Study 1A and 1B demonstrated that the relationship between SDO and environmentalism is weaker among student samples than general population samples. Therefore, the reliance on a student sample in Study 2A could explain why my hypothesis was not supported. However, the moderating effect of sample type has so far only been demonstrated cross-sectionally. Study 2A provides some evidence that the moderating effect may also exist over time. Replicating the longitudinal model within a general population sample is the next step to test this possibility.

In sum, Study $2 \mathrm{~A}$ is limited both in its reliance on a student sample and because the time between measurement points was only five months. To continue with this line of work in Chapter 3, Study 2B aims to address these limitations. 


\section{Study 2B}

\section{The influence of social dominance orientation and right-wing authoritarianism on environmentalism: A five-year cross-lagged analysis ${ }^{5}$}

\section{Introduction}

In the first analysis of how SDO and RWA relate to environmentalism over time, Study 1A shows that RWA is a significant longitudinal predictor of climate change denial and (marginally) of pro-environmental attitudes, whilst SDO is not. This research was conducted in a student population, and spanned only five months, thus warranting follow-up with a general population sample over a greater period of time. Fortunately through collaboration, I gained access to a national probability sample dataset (see below), where a representative sample of New Zealanders were followed up annually for five years.

As the main difference between Studies 2A and 2B is sample, I consult my meta-analyses in Chapter 2 again to inform my hypotheses. The meta-analyses showed that in general population samples, the association between SDO and environmentalism (while controlling for RWA) tended to be the stronger than in student samples. Although this result was determined from synthesizing crosssectional research, I argue that ideology does indeed precede attitudes (e.g. Duckitt, 2001), and predict that I will find a significant lagged effect of both RWA and SDO on environmentalism in this sample.

${ }^{5}$ Note. Study 2B has been rewritten for inclusion in the thesis from the manuscript submitted for publication:

Stanley, S. K., Milfont, T. L., Wilson, M. S., \& Sibley, C. G. (2019). The influence of social dominance orientation and right-wing authoritarianism on environmentalism: A fiveyear cross-lagged analysis. PLoS ONE 14(7): e0219067. https://doi.org/10.1371/journal.pone.0219067. 


\section{Method}

\section{Participants and Procedure}

The New Zealand Attitudes and Values Study (NZAVS) is a longitudinal study of personality, social attitudes, and health outcomes. It relies on data from a large, nationally representative group of people living in New Zealand who were sampled from the electoral roll. In this study, I utilize data from 22,966 individuals involved in the first five waves of data collection (from Time 1, collected in 2009 to Time 5, 2013). On average, participants were 43 years old when they began participating $(M=43.07, S D=14.67)$ and $62 \%$ were female. The majority of participants were born in New Zealand (76\%) and identified as Pākehā (New Zealand European; 86\%).

In Sibley's (2014) report on the sampling procedure of the NZAVS, he explains that participants were recruited by mail, with approximately $1.36 \%$ of all registered voters being mailed a questionnaire, and $16.6 \%$ of these individuals responded at Time 1 . The researchers retained $68 \%$ of these participants at Time 2, $60 \%$ at Time $3,62 \%$ at Time 4 (but $84 \%$ of the Time 3 sample), and $60 \%$ and Time 5 ( $81 \%$ of Time 4 ), introducing booster samples from Time 3 onwards to compensate for attrition. The NZAVS website includes more details of the sample, as well as further information about the procedure, retention rates, and demographics of participants: https://www.psych.auckland.ac.nz/en/about/our-research/researchgroups/new-zealand-attitudes-and-values-study.html.

\section{Measures}

As the NZAVS is a large scale survey, measuring hundreds of constructs per time point, the researchers tend to adopt shortened scales to measure the constructs of interest. From Chapter 2, I note that this does not influence the strength of the relationship between these ideological variables and environment-relevant variables. In Table 3.2, I present the reliability information for the SDO and RWA scales. The scales achieved poorer reliability than is typically found when full SDO and RWA scales are employed, however the Cronbach's alphas were consistent with existing research on these shortened scales (e.g., Pratto et al., 2013). 
Social dominance orientation. SDO was assessed using a shortened 6-item version of Sidanius and Pratto's (1999) SDO$_{6}$ scale. The subset of items included three items tapping the dominance component of SDO, e.g. "To get ahead in life, it is sometimes okay to step on other groups", and three favoring the equality dimension, e.g. "We should have increased social equality". For both SDO and RWA scales included in the NZAVS, responses were on a 7-point Likert scale from 1 (Strongly disagree) to 7 (Strongly agree).

Right-wing authoritarianism. Participant's level of RWA was assessed using a shortened version of Altemeyer's (1996) RWA scale. In Time 2, the item "Some of the best people in our country are those who are challenging our government, criticizing religion, and ignoring the "normal way" things are supposed to be done" was inadvertently excluded from the survey, thus Time 2 included just 5 items to measure RWA. At all other time points, a 6-item balanced scale was employed.

Environmentalism. The measure of environmentalism employed in this study is willingness to make sacrifices for the environment. This was measured using two items modeled from Liu and Sibley (2012), "Are you willing to change your daily routine in order to protect the environment?" and "Are you willing to make sacrifices to your standard of living (e.g., accept higher prices, drive less, conserve energy) in order to protect the environment?" For these items, responses were on a scale from 1 (definitely no) to 7 (definitely yes), with a midpoint of 4 (maybe).

\section{Data Analysis}

Consistent with my analyses in Study 2A, I used SPSS (IBM, version 23) to compute descriptive statistics and Mplus (version 7.3; Muthén \& Muthén, 1998-2010) to test the cross-lagged panel model. The model was comprised of three latent variables at each time point (SDO, RWA, and willingness to make sacrifices for the environment). In the main model, paths related each variable of interest at each time point to test the autoregressive paths (the stability of each variable over time) and cross-lagged paths (how the variables effect each other over time). I employed full information maximum likelihood to impute any missing data, and applied MLR for robust standard errors for our analysis. 
Because of the size of the dataset and multiple time points included, I employed additional constraints in this model. I used a stationary cross-lagged model by constraining each parameter to be time-invariant (e.g. Osborne, Milojev \& Sibley, 2017). This was achieved by firstly constraining factor loadings for each latent variable to equality. This meant that the items making up each latent variable were constrained to equality over time. Put concretely, this saw the factor loading of item 1 on the latent variable 'SDO' as equal across all time points, as with item 2, et cetera, thus factor loadings did not change across time points. Next, the means (intercepts) of each latent variable were constrained to equality across time, such that the latent mean of SDO, RWA, and environmentalism were invariant from Time 1 to Time 5. Furthermore, the residual variance for each item over time was constrained to equality. This meant that item 1 for SDO had equal variance across measurement points. Finally, I constrained to equality all cross-lagged associations.

These constraints, which made the model a stationary cross-lagged panel model, were employed because the focus of Study 2B was on the effects of SDO and RWA on changes in environmentalism across time, both overall and comparatively. Therefore, I was not interested in (and did not expect) any differences in the associations between measurement points. I assess fit of this model according to the same fit statistics in Study 2A.

To assess my hypotheses, the model gives unstandardized parameters that are invariant across time points (because of the constraints placed on the model). Kim and Mueller (1976) recommend examining the unstandardized estimates when comparing causal relationships, despite the convention of standardized estimates being considered more interpretable. To ease interpretation of the strengths of the paths, I present both the unstandardized parameters, which are invariant across time points, and the standardized paths, which are comparable. While comparing standardized paths can point to which variable is the stronger predictor, traditionally a Wald test is computed to assess if this difference is statistically significant. However, the Wald test is not possible in this circumstance given the model complexity involving multiple constraints. To get around this, I repeated analysis of the main model after manually adding similar constraints as in the Wald test. Specifically, I constrained the SDO-environmentalism and RWA-environmentalism paths to equality and compared 
the fit to the main model. When the models have equivalent fit (i.e. a non-significant Chi-Square difference test), this is taken as evidence that the variables are not statistically different predictors. When there is a significant improvement in fit (again determined by the change in Chi-Square), then this means that there is a statistical difference in the predictive ability of the variables.

Finally, because this study draws on data from over twenty thousand participants, I rely on the more conservative threshold of $p<.01$ to assess significance of findings (see also Milojev, Osborne \& Sibley, 2014). This minimizes the risk of rejecting the null hypothesis inappropriately (i.e. a Type 1 error) due to the high power of these data being more likely to return significant results.

\section{Results}

I present the descriptive statistics for the first time point in Table 3.2. Similar to the student sample used in Study 2A, on average participants endorsed low levels of SDO and RWA, and scored relatively highly in willingness to make sacrifices for the environment. The association between SDO and RWA was comparatively weaker in this sample, yet both ideological variables exhibit the expected negative relationships with environmentalism. The zero-order correlation is notably stronger between environmentalism and SDO than with RWA, and the confidence intervals for these estimates do not overlap (thus implying SDO is a significantly stronger correlate). 
Table 3.2.

Descriptive statistics and correlations between variables across all time points.

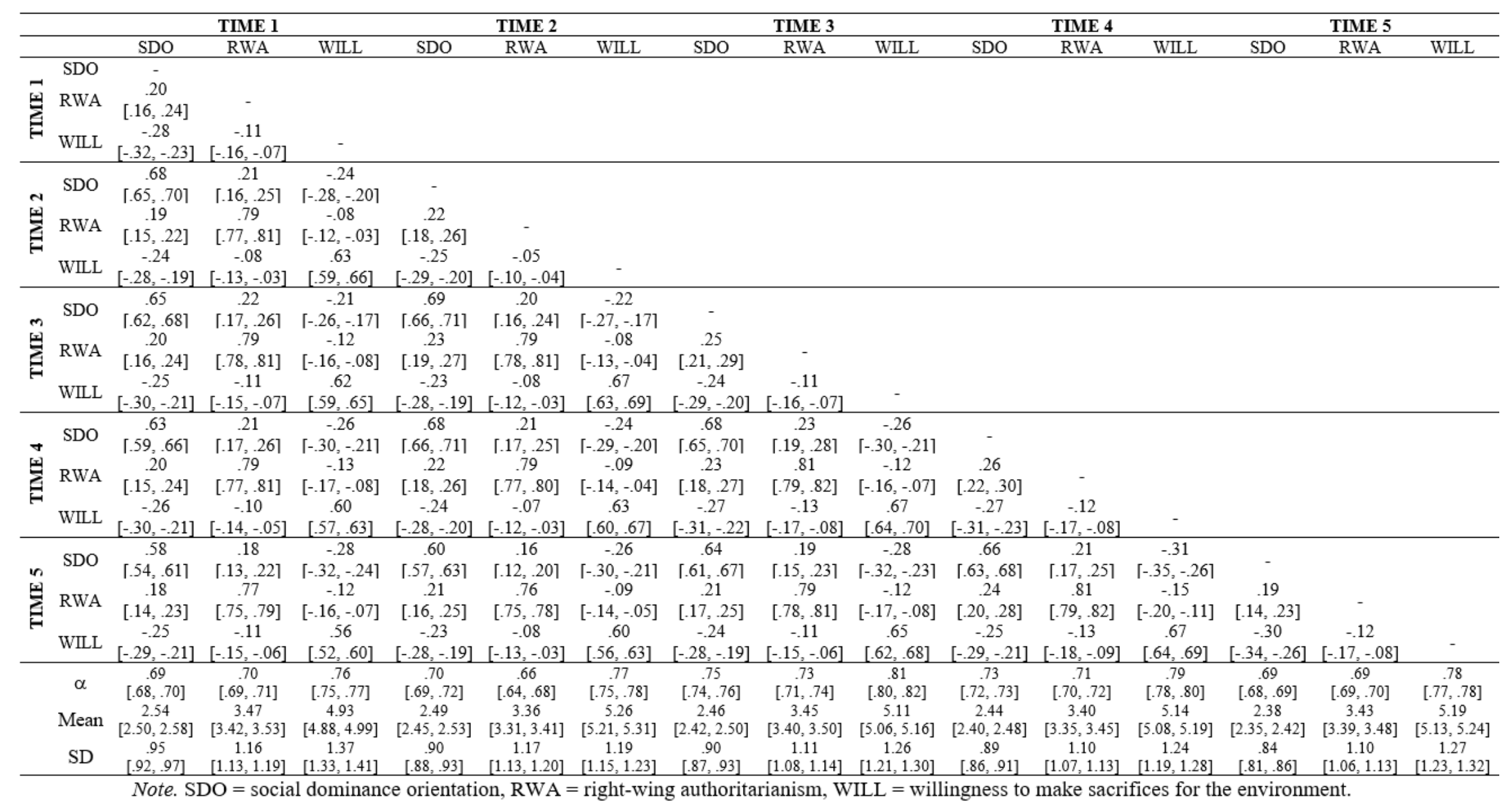

Numbers in square brackets represent $95 \%$ confidence intervals based on 1000 iterations. These values are based on participants who completed all items at all time points only $(\mathrm{N}=2041)$. 
Model fit. This model is extremely complex and contains data from a large number of participants. Recall from the model fit criteria in Study 2A, this means that fit is likely to be poor (at least in the case of the Chi-Square and CFI tests). Indeed, the Chi-Square test was significant $\left(\chi^{2}(2279)=36519.58\right)$, and the value remained considerably high when taking into account the degrees of freedom in the model $\left(\chi^{2} / \mathrm{df}=16.02\right)$. Furthermore, the CFI did not exceed the desired .95 threshold $(\mathrm{CFI}=$ .86). Despite this, the SRMR was appropriately low (.07), and RMSEA indicated excellent fit $(.03,90 \%$ CI $[.03, .03])$, and therefore overall, I judged the model to be acceptable.

The fit of a stationary cross-lagged model can be improved by parceling factor loadings (as in Osborne et al., 2017). I judged that this was not necessary as the main goals of this study were to test the strengths of relationships, not to achieve perfect fit, and therefore decided not to take steps to (perhaps artificially) improve fit using parceling. Furthermore, the technique is sometimes problematic if used on scales that are not unidimensional, as is the SDO scale (e.g. Jost \& Thompson, 2000) and some argue the RWA scale (Mavor, Louis \& Sibley, 2010), although the multidimensionality of this construct is clearer when measured using the ACT scale (Duckitt et al., 2010). As a final point, creating parcels would also prove challenging for the RWA scale as one item was excluded from Time 2, meaning that I could not create parcels that would be identical over the five time points. For a further discussion of when parceling is and is not recommended, see Little, Cunningham, Shahar and Widaman (2002).

Stability. To ascertain how stable the variables were over time, I examine the autoregressive paths in Figure 1. Across time points, participants mostly retained their level of SDO and RWA, which is consistent with arguments that ideology is largely steady over time $\left(\mathrm{B}_{\mathrm{SDO}}=.79, \mathrm{SD}=.01, p<.001,95 \% \mathrm{CI}[.78, .80], \beta\right.$ 's range from $.78-.83 ; \mathrm{B}_{\mathrm{RWA}}=.95, \mathrm{SD}=.003, p<.001,95 \% \mathrm{CI}[.94, .96], \beta$ 's range from .97-.98). Similarly, there was strong stability in participant's willingness to make sacrifices for the environment $(\mathrm{B}=.83, \mathrm{SD}=.01, p<.001,95 \% \mathrm{CI}[.82, .84], \beta$ 's range from .83$.87)$.

Association between SDO and RWA over time. I did not replicate the pattern of results from Study 2A of a bidirectional association between SDO and 
RWA. Instead, I found that SDO and RWA relate only in one direction over time. Specifically, RWA is a significant predictor of changes in SDO over time $\left(\mathrm{B}_{\mathrm{RWA}}=\right.$ $.02, \mathrm{SD}=.003, p<.001,95 \%$ CI $[.02, .03]$, all $\beta$ 's .03), whilst SDO does not predict change in RWA ( $\mathrm{B}_{\mathrm{SDO}}=-.001, \mathrm{SD}=.01, p=.844,95 \% \mathrm{CI}[-.01, .01], \beta$ 's -.001). Imposing Wald-like restraints, I find that RWA is a significantly stronger predictor of SDO than SDO is of RWA $\left(\Delta \chi^{2}(1)=11.46, p<.001\right)$ 


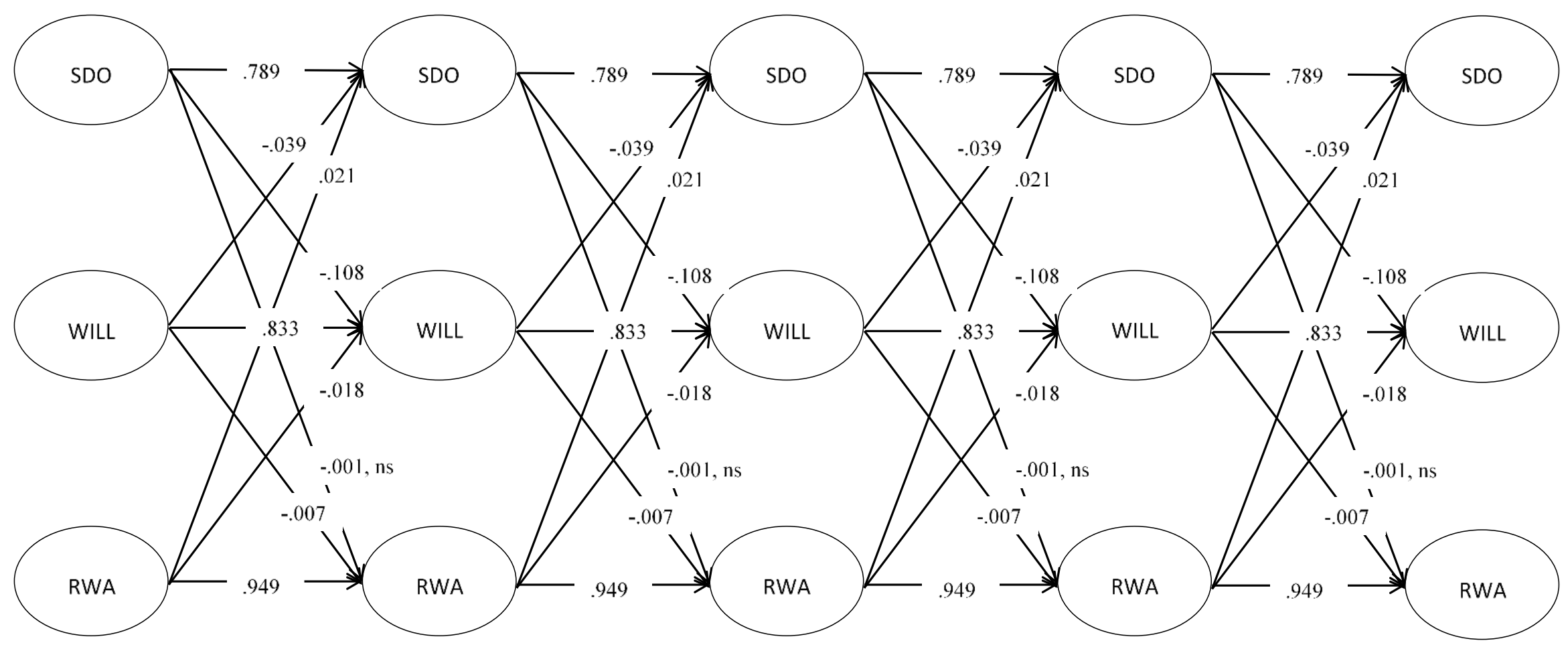

Figure 3.2. Unstandardized parameters assessing the association between social dominance orientation (SDO), right-wing authoritarianism (RWA), and willingness to sacrifice for the environment (WILL). All meet the accepted threshold for significance of $p<.01$ unless otherwise noted. 


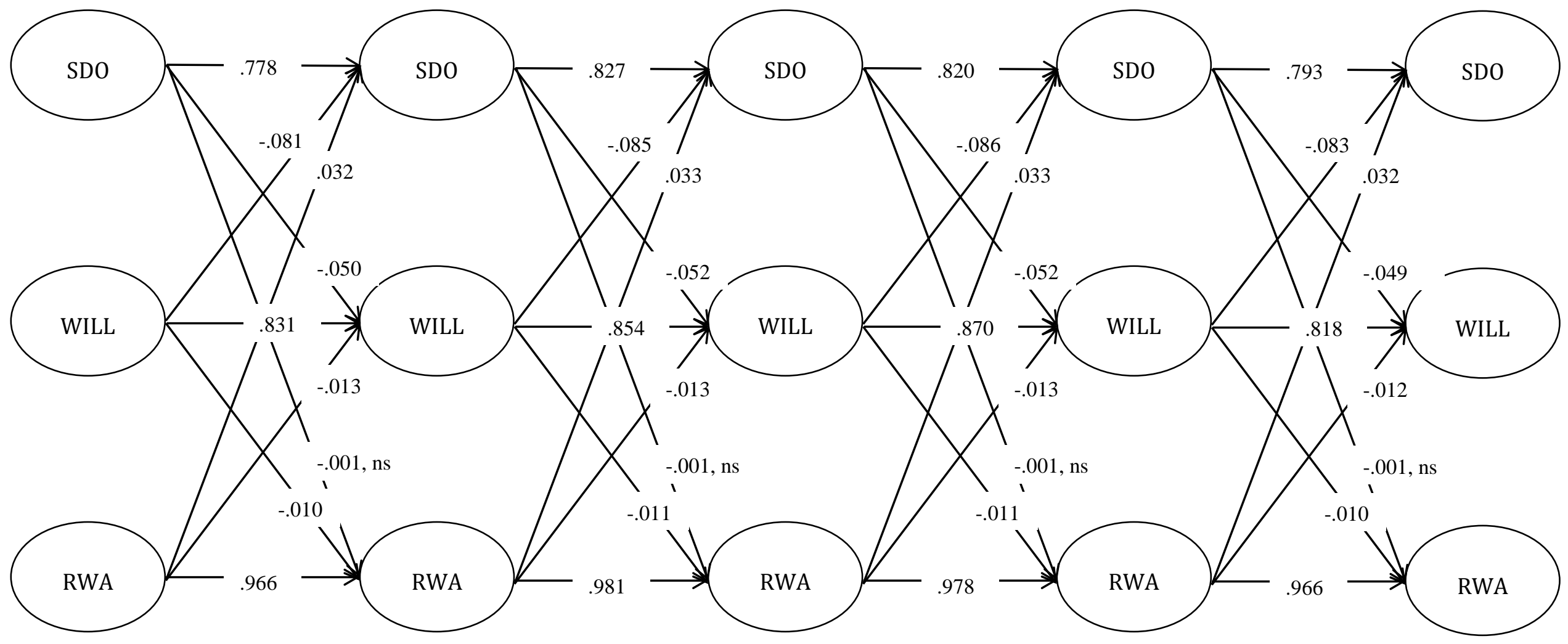

Figure 3.3. Standardized parameters assessing the association between SDO, RWA, and willingness to sacrifice for the environment (WILL). All meet our accepted threshold for significance of $p<.01$ unless otherwise noted. 
Relationship between ideology and environmentalism. I expected that SDO and RWA would predict increased environmentalism, here defined as willingness to make sacrifices for the environment. Indeed, Figure 1 shows that both SDO (B = -.11, $\mathrm{SD}=.01, p<.001,95 \% \mathrm{CI}[-.13,-.09]$, all $\beta$ 's -.05) and RWA $(\mathrm{B}=-.02, \mathrm{SD}=.01, p$ $=.001,95 \% \mathrm{CI}[-.03,-.01]$, all $\beta$ 's -.01$)$ relate to decreased pro-environmental intention over time. Next, I wanted to test whether they were equivalent predictors (consistent with the meta-analytic cross-sectional results), or if one ideology was a statistically stronger longitudinal predictor than th other. My first hint that this is the case is by examining the standardized estimates, and I note that SDO appears to be the stronger predictor, and the confidence intervals surrounding the standardized estimates do not overlap. After imposing constraints on the model, this is confirmed: SDO is a statistically stronger predictor of environmentalism than RWA $\left(\Delta \chi^{2}(1)=\right.$ $51.86, p<.001)$.

Although I did not make a specific prediction about the other direction of this association, the model does include a path from environmentalism to the ideological variables, and it is indeed significant. Expressing a willingness to make sacrifices for the environment is related to a small decrease in $\mathrm{SDO}(\mathrm{B}=-.04, \mathrm{SD}=.002, p<.001$, 95\% CI [-.04, -.03], $\beta$ 's between -.08 and -.09) and RWA (B = -.01, SD = .002, $p=$ $.002,95 \%$ CI [-.01, -.003], all $\beta$ 's -.01) over time. Therefore, the effect of ideology on environmentalism is bidirectional, and environmental attitudes do indeed influence endorsement of SDO and RWA. Furthermore, environmentalism more strongly predicts changes in SDO than in RWA $\left(\Delta \chi^{2}(1)=97.31, p<.001\right)$.

While the association between SDO and environmentalism was bidirectional, the lagged effect of environmentalism on SDO was stronger than the reverse $\left(\Delta \chi^{2}(1)\right.$ $=40.76, p<.001)$. This analysis helps to disentangle the directionality of the relationship by indicating that environmentalism has a stonger influence on SDO over time than SDO does on environmentalism. In contrast, there was no difference between the strength of the effect of RWA on environmentalism and vice versa $\left(\Delta \chi^{2}(1)=2.58, p=.108\right)$.

Explanatory power of the model. One final point is made by examining the amount of variance the model explains in our Time 2 variables (from the $\mathrm{R}^{2}$ values). 
Together, the variables at Time 1 explain between $67.4-75.8 \%$ of the variance in SDO, an extraordinary $93.5-96.6 \%$ of the variance in RWA, and $70.7-79.8 \%$ of the variance in environmentalism. These are likely driven mainly by the high autocorrelations.

\section{Comments}

My focus in this study was to determine whether and how SDO and RWA relate to environmentalism over time in a general population sample. I show that both ideologies are significant prospective predictors of willingness to make sacrifices for the environment, with SDO a statistically stronger predictor than RWA. This suggests that while both ideological variables may be foundational for later development of environmental attitudes, the preference for inequality explains more unique variance in changes in environmentalism. This highlights that, within the general population, Social Dominants are a potential target for climate change communications, as they are perhaps among those least motivated to act on the problem. However, environmentalism also significantly related to changes in ideology over time, and while there was no difference in the strength of the path from RWA to environmentalism and the reverse, contrary to hypotheses, I found that environmentalism is more strongly related to changes in SDO than the reverse.

This means that intentions to act pro-environmentally (by making sacrifices for the environment) decrease endorsement in SDO and RWA, thus the variables have reciprocal effects on one another. With the strong link between SDO, RWA, and prejudiced attitudes, it is interesting to note that boosting support for the environment could have flow-on longitudinal effects on reducing prejudice (and other 'consequences') by minimizing endorsement in these ideologies. Perhaps as well as motivating action on environmental issues, reducing endorsement of inequality and social hierarchy can broaden to effect other pro-social behaviors and attitudes, alluding to Duckitt's (2001) findings on the generality of prejudice.

One particularly interesting pattern of results is that of the associations between SDO and RWA. I found that RWA significantly predicted an increase in endorsement of SDO over time, while prospective SDO was unrelated to changes in RWA. This is especially impressive given the large sample size, where even very 
small associations are usually significant. Guided by Duckitt et al.'s (2002) research, this is interpreted as meaning that New Zealanders are likely more strongly socialised into authoritarianism than social dominance. Similar to the NEP-climate change denial association in Study 2A, an alternative explanation for the nonsignificant SDOto-RWA path is that there was little variance remaining to be explained in RWA, given the strong autoregressive path.

Study 2B used a powerful longitudinal dataset to show that SDO and RWA predict environmentalism over time, and vice versa, with SDO the stronger longitudinal predictor. The take home message of this study is that in general population samples, SDO is the key prospective predictor of willingness to make sacrifices for the environment. However, this research also questions the assumed temporal direction of the SDO-environmentalism link, and instead places environmentalism as the more likely antecedent of later ideological attitudes. 


\section{Discussion of Studies 2A and 2B}

While a small but growing literature on the associations between ideology and environmentalism have demonstrated the importance of understanding the role of SDO and RWA in environmental attitudes, Studies $2 \mathrm{~A}$ and $2 \mathrm{~B}$ are the first to empirically test the potential causal nature of these associations. In Study 2A, I showed that RWA is a stronger longitudinal predictor of changes in environmentalism than SDO in a student sample. Study 2B utilizes a general population sample to answer a similar question, and instead finds SDO to be the superior predictor.

On the surface, these represent two conflicting sets of results. However, the differences are reconciled by considering the findings from the meta-analyses reported in Chapter 2. These demonstrate that overall, SDO and RWA have comparable zero-order correlations and independent contributions to predicting environmentalism, though the SDO-environmentalism link is not equivalent across samples. Instead, the association with SDO is dependent on sample type, with SDO relating more strongly to environmentalism in general population samples than in student samples. This potentially explains why over time, SDO is more strongly related to increases in environmentalism in Study 2B than in Study 2A.

One further difference between the studies is in the way that each indexed environmentalism. In Study 2A, I included pro-environmental attitudes and climate change denial, whilst Study 2B relied on a measure of individual's willingness to make sacrifices for the environment. Overall, many of the aspects of environmentalism included in the meta-analyses in Chapter 2 had roughly equivalent associations with ideological attitudes, which points to the difference in sample as a more likely explanation for the different results.

Campbell (1963) introduced cross-lagged panel models as a way of testing causal associations between variables. When one lagged effect is stronger than the other, this is taken as evidence of a directional effect (Kenny \& Harackiewicz, 1979). This is the case in Study 2B for the relationship with SDO, where environmentalism is a stronger predictor of later SDO than the reverse, therefore suggesting that over time, environmentalism has a stronger effect on ideology. Where there was no significant difference between the lagged effect of RWA on environmentalism and the 
effect of environmentalism on RWA, this is potentially indicative of a spurious finding.

The stronger environmentalism-SDO directional relationship is counter to predictions, which were that ideology would more strongly relate to changes in environmental attitudes across time. It is possible that the refusal to make personal sacrifices for the environment leads individuals towards preferring an unequal society. Inaction on climate change means accepting its consequences and allowing these to fall to low status groups. It is possible that rejecting environmental action, when doing so is likely to exacerbate problems for low status groups, evokes feelings of cognitive dissonance. These are resolved through accepting social hierarchy and inequality as legitimate and fair (i.e., holding a social dominance orientation). Therefore, individuals who tolerate the environmental inequality brought on by the current environmental crisis, by way of forgoing making personal sacrifices to mitigate the problem, are more likely to extend these attitudes to general tolerance for social inequality.

Jylhä (2016) advanced the idea that individuals who endorse SDO are more likely to reject the reality of climate change as they are also more tolerant of the inherent inequity in both the causes and consequences of climate change. Denial may therefore serve as a way of maintaining or enhancing the status difference between social groups. My research runs parallel to this interpretation, by suggesting that SDO may serve as a way of protecting the individual from discomfort brought about by the social consequences of inaction on climate change.

There are some criticisms in the ability to draw conclusions about causal associations from CLPM (e.g. Hamaker, Kuiper \& Grassman, 2015). Arguably a more definitive test of causality would involve an experimental design. Such a design would divide participants at random, introduce one group to a manipulation set to increase or decrease SDO, and compare later environmental attitudes to a group who were not subjected to such a manipulation. However, given that my variables of interest have important implications for prejudice (e.g. Sidanius \& Pratto, 1999; Altemeyer, 1981), experimental research could be ethically dubious. Increasing SDO to examine the resultant effect on environmental issues risks a host of other consequences for intergroup relations. At this time, I am aware of few interventions 
that have succeeded in reducing levels of SDO (Zhao et al, 2018), which should increase pro-environmentalism if the variables are causally related. This is an interesting avenue for future research, and possibly could be achieved by targeting precursor beliefs (e.g. the belief that the world is a competitive place).

Cross-sectional research has established SDO and RWA as significant correlates of environment-relevant variables (see Chapter 2). In Chapter 3, I confirm this pattern of results exists across time. While longitudinal panel models cannot prove that the variables are causally related, they do show ideology to prospectively predict changes in environmentalism, both over a short, five month period (for RWA), and when tested annually over five years (both ideological variables). These findings support research applying social dominance theory and authoritarianism to aid our understanding of human-environment relations. They show that the ideological variables - while designed to help explain intergroup relations - can be broadened and understood as ideological foundations of environmental attitudes. In each study, the sizes of the lagged effects are small. However, this is unsurprising, given the slow rate of change of each variable (evidenced by the strong autoregressive paths).

While Study 2A demonstrated a bidirectional association between SDO and RWA, Study 2B found that only RWA predicted increased endorsement in SDO over time and not vice versa. Duckitt et al. (2002) explains that the SDO-RWA link is dependent on the dominant ideology in a particular sociopolitical context. When the dominant ideology is authoritarian-conservatism, then RWA predicts SDO more strongly, and the influence of SDO is either weak or nonsignificant. This is the pattern of results I found in Study 2B, thus suggesting that New Zealanders have generally been socialized into a context of authoritarianism. While both studies recruited New Zealanders as participants, the pattern was different for the student population in Study 2A: instead, both SDO and RWA reinforced each other over time. Duckitt (2001) notes that in young, student samples, SDO and RWA tend not to be related. Instead, their association emerges in adulthood, with a stronger correlation in adult samples.

However, there are cross cultural differences: In New Zealand, there tends to be a stronger association between SDO and RWA. Duckitt's (2001) attributes the stronger relationship in New Zealand to our society being more highly ideologized, 
with political issues tending to range on a strict continuum from left to right. Students sampled in Study 2A were therefore socialized into an ideologized context, but not in

such a way that one ideology was more prevailing than the other, hence explaining the lack of directional differences between SDO and RWA over time (Duckitt et al., 2002).

One main strength of the studies presented in Chapter 3 is that they draw from both student and general population samples, using both short- and long-term follow ups. Despite this, a significant longitudinal association does not definitively represent a causal relationship. Time 2 variables are clearly unable to retrospectively influence Time 1 variables, and thus ideology must flow in a forwards direction. It is possible that a third variable drives these temporal associations. For example, an academic who reviewed a version of this research submitted to Personality and Social Psychology Bulletin noted that those affected by economic downturn exhibit less favourable outgroup attitudes, and this could also drive a move away from proenvironmental attitudes. Economic downturn may indeed foster perceptions of competition for resources and thus SDO. Could it also predict a selfish approach to the environment?

Noting that changes in the predictor variables relate to corresponding changes to dependent variable supports a causal explanation of my findings, providing they are nonspurious, meaning that the association is not driven by variation in a third variable (Bachman \& Schutt, 2013). One further possibility is that socio-economic status is associated with both SDO and environmentalism, which is yet to be explored as a potential explanation for why the relationship exists. Consulting the literature, it is unclear what other potential third variable could be driving the ideologyenvironmentalism relationship, though future research ought to keep this possibility in mind and be wary in making causal claims.

\section{Conclusion}

In Studies 2A and 2B, I explored the longitudinal associations between SDO, RWA and environmentalism, finding that RWA is the stronger prospective predictor of environmentalism in Study 2A, yet this pattern is reversed in Study 2B, instead showing SDO to more strongly relate to changes in environmentalism. I tentatively 
explain the differences in the main findings in Chapter 3 as more likely due to the population type sampled from (rather than the aspect of environmentalism measured).

These studies extend on cross-sectional research to show that SDO and RWA may indeed be important for shaping people's environmental attitudes. The central caveat is that RWA is the key predictor of environmentalism in a student population, whereas those in the general population draw on their preferences for inequality and hierarchy to a greater extent to inform their environmental attitudes. Furthermore, an interesting and unexpected finding is that environmentalism more strongly predicts changes in SDO than the reverse directionality. Taken together, the findings in Chapter 3 indicate that ideology is an important factor to consider in research on environmentalism, although the causality is so far unclear. Moving forward, I continue to explore the SDO-environmentalism link to help clarify the nature of this association. 


\section{Chapter 4}

\section{Dimensions of social dominance orientation and their associations with environmentalism 6}

\section{Introduction to Studies 3A, 3B \& 3C}

In chapters 2 and 3, I establish social dominance orientation (SDO) as an important correlate, and prospective predictor, of environmentalism. SDO even remains an important longitudinal predictor in general population samples when controlling for the related ideological variable, right-wing authoritarianism (RWA), despite the association typically being weaker among student samples. Consistent with Sidanius and Pratto's (1999) first introduction of SDO as comprised of a single dimension, in studies of the SDO-environmentalism, SDO has been included as a unidimensional construct. However, subsequent research has suggested that SDO is actually comprised of two distinct dimensions (Jost \& Thompson, 2000).

With SDO defined as both the preference for social hierarchy and inequality (Sidanius \& Pratto, 1999), it is possible to separate the two facets of SDO to form two measurable subscales. The 16 -item $\mathrm{SDO}_{6}$ scale assesses social dominance using eight items that are worded in the direction where agreement on the item indicates endorsement of SDO (i.e. pro-trait items), and eight items that express rejection of SDO (con-trait items). Representative pro-trait items include "Inferior groups should stay in their place" and "To get ahead in life it is sometimes necessary to step on other groups", and con-trait items are those such as "Group equality should be our ideal" and "No one group should dominate society".

Note that the pro-trait items appear to tap dominance-related attitudes, whilst the con-trait items center around equality, leading Jost and Thompson (2000) to

${ }^{6}$ Note. The three studies reported in this chapter are based on the publication:

Stanley, S. K., Wilson, M. S., Sibley, C. G., \& Milfont, T. L. (2017). Dimensions of social dominance and their associations with environmentalism. Personality and Individual Differences, 107, 228-236. 
qualify these as SDO-Dominance and SDO-Egalitarianism. Because of this conflation, it is therefore not possible to separate the direction of wording of the items (i.e. whether they are pro- or con-trait items) from the aspect of SDO the item taps: dominance or egalitarianism. In 2012, Ho et al. published an updated $\mathrm{SDO}_{7}$ scale which avoids these criticisms, and more clearly measures each distinct subdimension of SDO. They achieved this by revising the $\mathrm{SDO}_{6}$ scale to include an equal number of pro- and con-trait items to measure dominance-related aspects of SDO and egalitarianism-related aspects.

Ho et al. $(2012,2015)$ explain the egalitarianism dimension of SDO (henceforth referred to as SDO-E) as capturing opposition to group equality, whereas the preference for some groups to dominate over subordinate groups characterises the dominance facet of SDO (SDO-D). In the context of intergroup relations, Ho and colleagues show that SDO-D is associated with overt racism and aggression, where the dominant group is active in their oppression of subordinate groups. SDO-E instead relates to endorsement of legitimizing myths that justify inequality, as well as passive resistance to hierarchy-attenuating policy - instead, those who endorse SDO-E oppose policies that redistribute social power. Ho et al. (2015) suggest, "Whereas individuals high on SDO-D prefer dominance hierarchies where high power groups oppress and subjugate low power groups, and are willing to achieve this form of inequality by use of very aggressive measures, individuals high on SDO-E prefer hierarchies where resources are inequitably distributed, and which can be defended by anti-egalitarian ideologies.” (p. 1022).

Despite research supporting a link between SDO and environmentalism (see Chapter 2), no research to date has broadened the two-dimensional approach of SDO to predicting environmentalism. Prior to the introduction of the $\mathrm{SDO}_{7}$ scale, this dearth is in no small part due to the absence of an effective measurement tool. As discussed in the General Introduction, SDO was first conceptualized as a way to aid our understanding of intergroup attitudes (Sidanius \& Pratto, 1993, 1999). However, research in the environmental domain shows a clear link between endorsement of group-based inequality and climate change belief (see Chapter 2).

There are logical reasons that could explain why either SDO-D or SDO-E might drive the association between SDO (measured unidimensionally) and 
environmentalism. Specifically, the preference for human dominance over nature may explain why Social Dominants are less concerned about the environment and more willing to exploit natural resources, an explanation that is consistent with dominance aspects of SDO (SDO-D). Alternatively, SDO-E may be the driving force behind the association owing to Social Dominants keenness for dominant groups to receive a disproportionate allocation of natural resources, and therefore permitting environmental exploitation to achieve this end.

Without directly testing whether SDO-D and/or SDO-E underlies the SDOenvironmentalism association, some research has alluded to the possibility that environmental exploitation is motivated by either Dominant or Egalitarian components of SDO. Recall that SDO-D predicts a preference for group-based dominance, and to achieve this, support for aggression or violence towards subjugated groups (Ho et al., 2012; 2015). This is reminiscent of the Dominant Social Paradigm, which encompasses a worldview of human dominance over nature (Pirages \& Ehrlich, 1974). Proponents of the DSP argue that humans are entitled to utilize natural resources. Consistent with this view, Pratto et al. (1994) presented the earliest evidence of an association between SDO and opposition to pro-environmental policy, putting this down to Social Dominants rejection of policies that equalize conditions for humans and other species. Similarly, Milfont et al. (2013) argued that the relationship exists because those who endorse SDO feel that humans are superior to nature.

Highlighting Social Dominants greater preference for active oppression over other groups, Jackson et al. (2013; Study 4) show high-SDOs to choose to direct the environmental hazards of a manufacturing plant to poorer locations, despite their own social group reaping the benefits. The Dominance facet of SDO expresses approval of the use of force against other groups to achieve an end: "It's okay to 'step on other groups' to get ahead"; "Some groups are inferior" (among other items: Ho et al., 2012; 2015). In Jackson et al.'s scenario, and consistent with a dominance view of SDO, hierarchy is achieved through subjugating a lower-power group (Ho et al., 2015).

The dominance over nature theories, combined with limited empirical research, do provide some reason to anticipate that SDO-D might relate more strongly 
to environmentalism than SDO-E. However, SDO-E is more predictive of political conservatism (Ho et al., 2015), which is one of the correlates of climate change denial (Hornsey et al., 2016). Furthermore, relative to SDO-D, Ho et al. (2015) showed SDO-E to more strongly relate to support for hierarchy-enhancement in the form of allocating more resources to those at the top of the hierarchy, and opposition to hierarchy-attenuating policy by objecting to those policies aimed at promoting groupbased equality.

In the environmental sphere, Milfont and Sibley (2014) theorized that Social Dominants exploit the environment to ensure their group benefits. Specifically, their hierarchy enhancing hypothesis of environmental exploitation contends that highSDO's can exploit the environment in their favour, with the end goal of achieving unequal distribution of resources. This is more consistent with the SDO-E facet of SDO. Support for this theory comes from Milfont and Sibley's finding that highSDO's will put their support behind a mining operation, but only if this will provide more benefits to the dominant group, thus increasing inequality. Similarly, Jackson et al. (2013, Study 2) show Social Dominants to support environmental exploitation when the benefits go to their own country only. While both studies treat SDO as a unitary construct, their conclusions allude to a more anti-Egalitarian take on SDO, where hierarchy is maintained through unequal distribution of resources (Ho et al., 2015).

Taken together, these findings suggest that assessing SDO-D and SDO-E as distinct subconstructs of SDO will help tease out the nature of the association between SDO and environmentalism. The purpose of the research presented in Chapter 4 is to test whether the two components of SDO differentially predict attitudes towards the environment and climate change beliefs. In Study 3A, I use an earlier measure of SDO to determine how this separates into the two dimensions, and how these relate to environmental attitudes in a general population sample. In Study 3B, I repeat this analysis with a dataset which employed the $\mathrm{SDO}_{7}$, and examine the underlying factor structure and associations with environmentalism in a student sample. In Study 3C, I replicate and extend on Study 3B by also testing the cross-time associations. 


\section{Study 3A}

\section{Separating the dimensions of social dominance orientation to predict environmentalism in a general population sample}

\section{Introduction}

Given the differential effects of SDO-D and SDO-E on attitudes relevant to intergroup relations, Study $3 \mathrm{~A}$ is the first to test the distinct effects of the dimensions of SDO on environmentalism. I do this using data from Time 1 of the same nationally representative sample of New Zealanders used in Study 2B, from the New Zealand Attitudes and Values Study (NZAVS). Bergh, Sidanius and Sibley (2015) also used these data to show that SDO-D and SDO-E differentially relate to socio-political attitudes. They found that SDO-D was predictive of sexist attitudes, whilst SDO-E related more strongly to conservative political orientation.

As discussed in the Introduction, and consistent with arguments by Pratto et al. (1994) and Milfont et al. (2013), SDO might primarily influence environmental attitudes through Social Dominants preference for human dominance over nature. Consistent with this argument, one expectation is that SDO-D will more strongly underlie environmental attitudes. Alternatively, Milfont and Sibley's (2014) argument that Social Dominants utilize the environment as a way of maintaining the social hierarchy supports the opposite pattern of results, with SDO-E as the driving facet. Finally, it is also possible to expect that both SDO-D and SDO-E predict environmentalism to similar degrees. There are therefore three conflicting predictions about how the facets will relate to environmental attitudes, and I aim to test these in an exploratory first step in Study 3A.

The NZAVS indexed endorsement of SDO based on a short version of the $\mathrm{SDO}_{6}$ scale, meaning that it is not possible to disentangle completely direction-ofwording from item content. At the same time, previous researchers (e.g., Eagly, Diekman, Johannesen-Schmidt \& Koenig, 2004; Jost \& Thompson, 2000; Sears, Haley \& Henry, 2008) have computed scale scores for the pro-trait/dominance and con-trait/egalitarianism items separately, showing that the two facets differentially 
predict a variety of other social and political variables. A secondary aim of the series of studies reported in Chapter 4 is to validate the use of both the $\mathrm{SDO}_{6}$ and $\mathrm{SDO}_{7}$ scales as a two-dimensional. While Sidanius and Pratto $(1993,1999)$ were adamant that SDO tapped just one construct (and that the two factors typically extracted represent direction of wording only), recent advances indicate that it is indeed comprised of both SDO-D and SDO-E dimensions (e.g. Ho et al., 2012, 2015). In Study 3A, I compare the fit of the original one-dimensional model to the new twofactor model of SDO (as indexed by the $\mathrm{SDO}_{6}$ scale). I expect to replicate existing findings that the two-dimensional model provides a better fit to the data.

\section{Method}

\section{Participants and procedure}

Participants were those individuals who completed the first wave of data collection in the NZAVS in 2009 by returning a mail questionnaire. A total of 6516 participants (59\% female) were included in the sample, with a mean age of 48 years $(M=48.09, S D=15.75)$, and a predominantly New Zealand European ethnic identification (86\%). Further details about the sample and procedure are reported in the Study 2B Method section.

\section{Measures}

Social Dominance Orientation. Recall that SDO was assessed using a shortened version of Sidanius and Pratto's (1999) SDO 6 scale. Of the six items included, three assess SDO-D (e.g. "To get ahead in life, it is sometimes okay to step on other groups"), and three assess SDO-E (e.g. "We should have increased social equality"). Responses to these items were on a scale from 1 (strongly disagree) to 7 (strongly agree).

Environmentalism. I included four measures of environmentalism in Study 3A. Firstly, I assessed climate change belief using two related items, "climate change is real" and "climate change is caused by humans". This is rated on the same 7-point Likert scale as for SDO. Two aspects of environmental sacrifice from Liu and Sibley's (2012) scale were also included. Willingness to make sacrifices for the environment was measured using two related items ("Are you willing to change your daily routine in order to protect the environment?" and "Are you willing to make 
sacrifices to your standard of living (e.g. accept higher prices, drive less, conserve energy) in order to protect the environment?"). These items were reworded by changing the willingness stem to "Have you made changes/sacrifices" to form two items that measure Having made sacrifices for the environment. Participants express agreement to all sacrifice items on a scale from 1 (definitely no) to 7 (definitely yes), with the midpoint 4 (maybe). Finally, one item from Schwartz's (1992) value scale was used to measure environmental value. Participants were asked to rate on a scale with anchors of -1 (opposed to my values), 0 (not important), 3 (important), 6 (very important) and 7 (of supreme importance) the extent to which "Protecting the environment (preserving nature)" is a guiding principle in their lives.

\section{Statistical analysis}

As I use the same dataset as in Bergh et al.'s (2015) analysis on the predictive ability of SDO-D and SDO-E, I follow their method to use a robust maximum likelihood estimator for all structural equation models presented in this Chapter. Bergh et al. used CFA to confirm that the two-dimensional model of SDO provided a good fit to the data, however they did not compare this to the one-dimensional model. In this study, I first assess whether the $\mathrm{SDO}_{6}$ scale suits a unidimensional or twofactor solution by examining which provides the best fit to the data. These confirmatory factor analyses are constructed such that in the one-factor model, all items in the $\mathrm{SDO}_{6}$ scale load on one factor (latent variable), and in the two-factor model, the SDO-D items load to form one factor, and the SDO-E items load on a second, separate but correlated, factor.

To test competing predictions about the associations between SDO-D, SDO-E and environmentalism, I constructed a path model where latent variables of SDO predict latent variables of environmentalism (with the environmental value measure of environmentalism as an observed variable, as this was measured using a single item). I constructed all models in Mplus (version 7.3, Muthén \& Muthén, 19982010). Because of the large sample size of the NZAVS data, and therefore high power of these analyses, I used the conservative threshold of significance of $p<.01$ to indicate statistically significant findings (Milojev et al., 2014). I used the Wald test to determine whether there is a statistically significant difference in the predictive ability 
of SDO-D and SDO-E. When significant, this test confirms that the stronger predictor is a statistically stronger predictor.

I evaluated the fit of these models using similar indices as explained in the Method section of Study 2A. This includes the Chi-Square test (ideally nonsignificant, or less than 3 when divided by its degrees of freedom), CFI (ideally $>$ $.95)$, RMSEA $(<.06)$, SRMR $(<.08)$. Additionally, to compare the fit of two different models (i.e. when comparing between the unidimensional and two-factor model of SDO), the best model is the one that has a lower Akaike Information Criterion (AIC) value, and shows a significant improvement in the Chi-Square difference test. The difference test used in the models in Chapter 4 is the Satorra-Bentler scaled Chisquare difference (scaled $\Delta \chi^{2}$ ) test. This is a more conservative test, appropriate to use when employing a robust maximum likelihood estimator, and it adjusts the mean only if a non-normal distribution is detected (Satorra \& Bentler, 2010).

\section{Results}

\section{Confirmatory factor analyses}

In Table 4.1, I present the model fit for all confirmatory factor analyses conducted in Chapter 4 (For Study 3A, 3B, and 3C). Of note for Study 3A is the first line, which compares the fit of the unidimensional and two-factor solution to the $\mathrm{SDO}_{6}$ scale. This shows that the two-factor model provides significantly better fit to the data: scaled $\Delta \chi^{2}(1)=425.22, p<.001$. This justifies the treatment of the $\mathrm{SDO}_{6}$ scale as two-dimensional, and thus I continue with the model that separates SDO into its two dimensions to predict environmentalism. 
Table 4.1.

Model fit for one- and two-dimension models, two-method models, and four-factor models used in each study presented in Chapter 4.

\begin{tabular}{|c|c|c|c|c|c|c|c|c|}
\hline & $X^{2}$ & $d f$ & $X^{2} / d f$ & CFI & AIC & RMSEA & $\begin{array}{l}\text { 90\% CI } \\
\text { RMSEA }\end{array}$ & SRMR \\
\hline \multicolumn{9}{|l|}{ Study 3a } \\
\hline One-dimension model & 761.32 & 9 & 84.59 & .84 & $135,887.32$ & .11 & {$[.11, .12]$} & .07 \\
\hline Two-dimension model & 135.33 & 8 & 16.92 & .97 & $135,041.03$ & .05 & {$[.04, .06]$} & .03 \\
\hline \multicolumn{9}{|l|}{ Study 3b } \\
\hline One-dimension model & 421.30 & 104 & 4.05 & .86 & $26,412.34$ & .08 & {$[.07, .09]$} & .06 \\
\hline Two-dimension model & 284.83 & 103 & 2.77 & .92 & $26,230.84$ & .06 & {$[.05, .07]$} & .05 \\
\hline Two-method model & 297.14 & 103 & 2.88 & .92 & $26,248.35$ & .06 & {$[.05, .07]$} & .05 \\
\hline Four-factor model & 150.81 & 86 & 1.75 & .97 & $26,036.22$ & .04 & {$[.03, .05]$} & .03 \\
\hline \multicolumn{9}{|l|}{ Study 3c (Time 1) } \\
\hline One-dimension model & 374.59 & 104 & 3.60 & .90 & $36,484.34$ & .06 & {$[.06, .07]$} & .05 \\
\hline Two-dimension model & 268.84 & 103 & 2.61 & .94 & $36,354.40$ & .05 & {$[.04, .06]$} & .04 \\
\hline Two-method model & 321.07 & 103 & 3.12 & .92 & $36,419.50$ & .06 & {$[.05, .06]$} & .04 \\
\hline Four-factor model & 122.56 & 86 & 1.43 & .99 & $36,203.07$ & .03 & {$[.01, .04]$} & .02 \\
\hline
\end{tabular}

Note. $\chi^{2}=$ Chi Square, $d f=$ degrees of freedom, CFI = comparative fit index, RMSEA = root mean square approximation, 90\% CI RMSEA = confidence interval around RMSEA of the change in fit between models, AIC = Akaike Information Criterion, SRMR = standardized root mean square residual. 
Table 4.2.

Descriptive statistics, and correlation matrix between mean scores of each variable in Study $3 A$.

\begin{tabular}{|c|c|c|c|c|c|c|c|c|c|}
\hline & & Mean & SD & 1. & 2. & 3. & 4. & 5. & 6. \\
\hline \multirow{2}{*}{1.} & \multirow{2}{*}{ SDO-D } & 2.43 & 1.16 & $\alpha$ & & & & & \\
\hline & & {$[2.41,2.46]$} & {$[1.13,1.18]$} & .54 & & & & & \\
\hline \multirow{2}{*}{2.} & \multirow{2}{*}{ SDO-E } & 2.76 & 1.19 & $.34 * * *$ & & & & & \\
\hline & & {$[2.73,2.79]$} & {$[1.17,1.22]$} & {$[.32, .37]$} & .76 & & & & \\
\hline \multirow{2}{*}{3.} & \multirow{2}{*}{ Climate belief } & 5.09 & 1.49 & $-.08 * * *$ & $-.26 * * *$ & $\alpha$ & & & \\
\hline & & {$[5.05,5.13]$} & {$[1.46,1.51]$} & {$[-.11,-.05]$} & {$[-.28,-.23]$} & .70 & & & \\
\hline \multirow{2}{*}{4.} & \multirow{2}{*}{ Made sacrifice } & 4.69 & 1.55 & $-.12 * * *$ & $-.18 * * *$ & $.28 * * *$ & $\alpha$ & & \\
\hline & & {$[4.85,4.92]$} & {$[1.53,1.58]$} & {$[-.15,-.09]$} & {$[-.21,-.15]$} & {$[.25, .30]$} & .77 & & \\
\hline \multirow{2}{*}{5.} & Willing to & 4.89 & 1.40 & $-.15 * * *$ & $-.25^{* * *}$ & $.38 * * *$ & $.70 * * *$ & $\alpha$ & \\
\hline & sacrifice & {$[4.85,4.92]$} & {$[1.38,1.42]$} & {$[-.18,-.12]$} & {$[-.28, .23]$} & {$[.36, .41]$} & {$[.68, .72]$} & .76 & \\
\hline \multirow{2}{*}{6.} & Value & 5.12 & 1.55 & $-.10 * *$ & $-.21 * * *$ & $.30 * * *$ & $.39 * * *$ & $.46^{* * *}$ & $\alpha$ \\
\hline & environment & {$[5.13,5.20]$} & {$[1.52,1.58]$} & {$[-.13,-.08]$} & {$[-.24,-.19]$} & {$[.28, .33]$} & {$[.37, .42]$} & {$[.43, .48]$} & N/A \\
\hline
\end{tabular}

Note. Numbers in square brackets are 95\% confidence intervals based on bootstrapping of 1000 iterations. SDO-D = dominance sub-dimension of SDO. SDO-E = anti-egalitarian sub-dimension of SDO. Along the diagonal are Cronbach's alphas for the scales. $* * p<.01, * * * p<.001$. 


\section{Descriptive Statistics}

I present means, standard deviations, and Cronbach's alphas in Table 4.2. All alphas except that for SDO-D were above .7, and therefore point to acceptable reliability. I note that the alpha for SDO-D is lower, at .54, however this is not unusual for short scales (3 items), and as a further reliability test, the corrected interitem correlations range from .29 to .41 , which is close to ideal as recommended by Briggs and Cheek (1986). Also presented here are the correlations between the measures, which are in the expected directions: SDO-D and SDO-E are positively correlated, as are the environmental variables, and SDO-D and SDO-E relate negatively to pro-environmentalism.

\section{Path model}

In my main model, SDO-D and SDO-E are set to predict the environmentrelevant variables. Looking across the model fit indices, the model demonstrates adequate fit $\left(\chi^{2}(51)=747.26, p<.001 ; \chi^{2} / \mathrm{df}=14.65 ; \mathrm{CFI}=0.96 ; \mathrm{RMSEA}=0.05\right.$; SRMR $=0.03$ ) based on all indicators aside from the Chi-square test, which (consistent with Study 2b) is likely influenced by the large sample size. As displayed in Figure 4.1, SDO-E is consistently more strongly related to environmentalism than SDO-D.

Specifically, SDO-E is a stronger predictor than SDO-D of willingness to make sacrifices for the environment (SDO-E: $\beta=-0.30, p<.001,95 \%$ CI [-0.34, 0.25]; SDO-D: $\beta=-0.07, p=.004,95 \% \mathrm{CI}[-0.12,-0.02])$, and this difference is statistically significant as indicated by the Wald test (Wald test $(1)=15.83, p<.001)$. While SDO-E appears a stronger predictor of having made sacrifices for the environment (SDO-E: $\beta=-0.20, p<.001,95 \%$ CI [-0.24, -0.15]; SDO-D: $\beta=-0.09, p$ $<.001,95 \%$ CI $[-0.14,-0.04]$ ), the difference is not statistically different (Wald test(1) $=2.41, p=.121)$. However, SDO-E is a significantly stronger predictor of the environmental value of 'protecting nature' (SDO-E: $\beta=-0.23, p<.001,95 \% \mathrm{CI}[-$ 0.27, -0.20]; SDO-D: $\beta=-0.03, p=.212,95 \%$ CI [-07, 0.02]; Wald test $(1)=20.02, p$ $<.001$ ), and climate change belief (SDO-E: $\beta=-0.39, p<.001,95 \%$ CI $[-0.43,-$ 0.34]; SDO-D: $\beta=0.08, p=.002,95 \%$ CI [0.03, 0.12]; Wald test $(1)=95.94, p<$ $.001)$. 


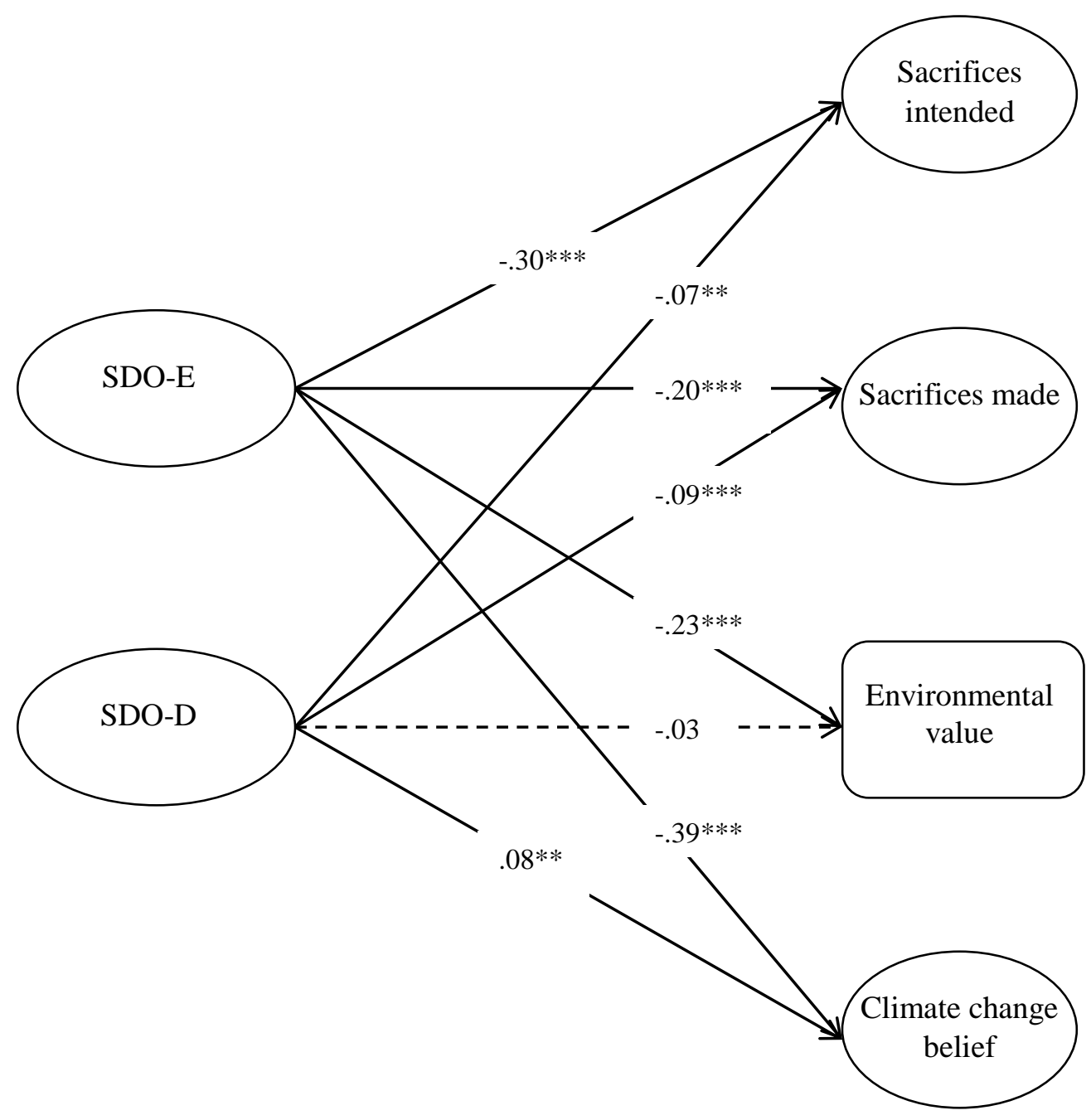

Figure 4.1. SDO-D (Dominance) and SDO-E (Egalitarianism) predicting environmental variables in Study 3A.

Note. Solid lines show significant paths $(* * p<.01, * * * p<.001)$ and dashed lines non-significant paths $(p>.01)$. 
Table 4.3.

Wald test results comparing significance of SDO-D and SDO-E as predictors of environment-relevant variables.

\begin{tabular}{|c|c|c|c|}
\hline $\begin{array}{l}\text { Environmentalism } \\
\text { measure }\end{array}$ & Value & $\begin{array}{l}\text { Degrees of } \\
\text { freedom }\end{array}$ & P-value \\
\hline \multicolumn{4}{|c|}{ Study 3A } \\
\hline Sacrifices intended & 15.83 & 1 & $<.001$ \\
\hline Sacrifices made & 2.41 & 1 & .121 \\
\hline Environmental value & 20.02 & 1 & $<.001$ \\
\hline Climate change belief & 95.94 & 1 & $<.001$ \\
\hline \multicolumn{4}{|c|}{ Study 3B } \\
\hline Sacrifices intended & 9.59 & 1 & .002 \\
\hline Sacrifices made & 0.51 & 1 & .474 \\
\hline Environmental value & 3.06 & 1 & .080 \\
\hline Climate change belief & 7.12 & 1 & .008 \\
\hline \multicolumn{4}{|c|}{ Study 3C (longitudinal) } \\
\hline $\begin{array}{l}\text { SDO predicting } \\
\text { Climate change denial }\end{array}$ & .12 & 1 & .900 \\
\hline SDO predicting NEP & .59 & 1 & .441 \\
\hline $\begin{array}{l}\text { Enviro predicting } \\
\text { SDO-D }\end{array}$ & 11.93 & 1 & .001 \\
\hline $\begin{array}{l}\text { Enviro predicting } \\
\text { SDO-E }\end{array}$ & 6.50 & 1 & .011 \\
\hline
\end{tabular}


The results of the Wald tests, as summarized in Table 4.3, show that SDO-E was a significantly stronger predictor of three of the four environment-relevant variables included in this model than SDO-D. This indicates support for the second of my competing predictions (and suggesting that the SDO-environmentalism link is better explained by Social Dominants preference for high-status groups inequitable use of the environment).

\section{Comments}

In Study 3A, I provided the first test of the two-factor model of SDO applied to environmental attitudes. This test relied on use of the $\mathrm{SDO}_{6}$ scale. Whilst this scale was designed to measure SDO as a unidimensional construct (Sidanius \& Pratto, 1999), I show that it does indeed separate into two distinct dimensions. Furthermore, these dimensions are not only statistically distinct but also meaningfully different in ways that do not clearly reflect direction-of-wording only, working to predict environmentalism in different ways (i.e. with one more strongly related than the other).

While previous studies have reported an association between SDO and environment-relevant variables, Study $3 \mathrm{~A}$ provides the first indication that this relationship is driven mostly by SDO-E, at least in this particular sample. Specifically, I show that SDO-E predicts all four aspects of environmentalism more strongly than SDO-D, and for three of these variables SDO-E is a significantly stronger predictor. This suggests that it is the opposition to group-based equality driving Social Dominants exploitation of the environment, and the preference for dominance is related to a lesser extent.

Study 3A used a shortened measure of SDO to examine the association with environmentalism. While I note in Chapter 2 that shortened measures estimate the relationship between SDO (as unidimensional) and environmentalism as well as fullscale measures, the shortened version of the $\mathrm{SDO}_{6}$ scale used here is problematic for this particular analysis. This is because, as noted already, all SDO-E items are contrait, and all SDO-D items are pro-trait. It could be that people who agree to con-trait items are more likely to endorse environmentalism. As it stands, the scale conflates 
wording direction and content (whether SDO-E or SDO-D is implied), thus I cannot be certain that this limitation does not influence the results.

As discussed earlier, the $\mathrm{SDO}_{7}$ has been developed to address these limitations (Ho et al., 2012, 2015). I therefore aim to replicate these findings in Study 3B using this scale specifically designed to measure SDO-D and SDO-E dimensions of SDO. 


\section{Study 3B}

\section{Examining how the two dimensions of social dominance orientation differentiate to predict environmentalism using the $\mathrm{SDO}_{7}$}

\section{Introduction}

Study 3A established that SDO-E is the main driver of the SDOenvironmentalism link, though it did so using a measure which is perhaps inappropriate to separate into the two dimensions. In Study 3B, I aim to replicate the pattern of associations using the full-scale $\mathrm{SDO}_{7}$. I use a large, student sample, and measure the same aspects of environmentalism as in Study 3A.

The findings of Study 3A supported the interpretation that anti-egalitarianism underlies the SDO-environmentalism association. I expect to find the same pattern of results in this study: SDO-E will likely be a stronger predictor of environmentrelevant variables than SDO-D. Furthermore, I expect the $\mathrm{SDO}_{7}$ to separate into four factors, with two wording direction factors (pro- and con-trait), and two content factors (SDO-D and SDO-E), to replicate Ho et al. (2015). I expect this model to provide the best fit to the data, followed by the two-factor model (SDO-D and SDOE, which I then use to test my main hypothesis), with the unidimensional model providing a relatively poor fit to the data.

\section{Method}

\section{Participants and procedure}

Five hundred and four first year psychology students participated in the study online. In exchange for completing the study, they received credit towards their required research participation hours. The Victoria University School of Psychology Human Ethics Committee approved this research prior to data collection (Ethics ID number 0000022835). 


\section{Materials}

My variables of interest were measured in one of several short 'packets' of studies. Each scale was presented in a randomized order, and the items within each scale were similarly randomized.

Social dominance orientation. I used Ho et al.'s (2012) $\mathrm{SDO}_{7}$ scale to assess SDO. As described in Study 2A, this measure includes eight items tapping SDO-D (with four pro-trait items, e.g. "Having some groups on top really benefits everybody"; and four con-trait: "No one group should dominate in society"), and a further balanced set of eight items to assess SDO-E (pro-trait: "We should not push for group equality"; con-trait: "Group equality should be our ideal"). Responses are recorded on a scale from 1 (strongly disagree) to 7 (strongly agree).

Environmentalism. I measured the same four aspects of environmentalism using the same scales as in Study 3A: climate change belief, willingness to make sacrifices for the environment, having made sacrifices for the environment, and environmental value.

\section{Statistical analysis}

My first set of analyses were the confirmatory factor analyses. I aim to replicate Ho et al.'s (2015) finding that the four-factor model of SDO provides the best fit to the data. This model takes into account wording direction (pro-trait and con-trait) as well as item content (SDO-D and SDO-E). In this model, pro- and contrait items formed two method factors, and the same items were also set to form SDOD and SDO-E factors. Therefore, each item loaded on both a method and a item content factor. The two-factor and unidimensional models were constructed in the same manner as in Study 3A.

Again following the analytical procedure described in Study 3A, I next constructed a path model where SDO-D and SDO-E are set to predict our four indices of environmentalism (with all variables - except environmental value - made up of latent variables). The sample is smaller in Study 3B, thus I return to the standard $p<$ .05 threshold when considering significance of findings. As in Study 3A, I use the Wald test to assess statistically significant differences in the strengths of the paths. 
Finally, goodness of fit is evaluated according to the same indices adopted in Study 3A.

\section{Results}

\section{Confirmatory factor analyses}

The fit statistics of my CFA models are included in Table 4.1. These demonstrate that the four-factor model of SDO, which is comprised of two factors of item wording (pro- and con-trait) and two content factors (SDO-D and SDO-E), provides the best fit to the data. This model was significantly better than the onefactor model (scaled $\Delta \chi^{2}(1)=71.52, p<.001$ ), therefore supporting the use of the $\mathrm{SDO}_{7}$ subscales. While the four-factor model provides the best fit to the data, the aims of the next step in my research was to determine how the content factors relate to environmental attitudes, and therefore I disregard the method factors for the next set of analyses to use SDO-D and SDO-E as predictors.

\section{Descriptive statistics}

Descriptive statistics, including means, standard deviations, and reliability information for each scale are presented in Table 4.4. Also presented here are the correlations between variables, which replicate the pattern of zero-order correlations reported in Study 3A. Specifically, the SDO-D and SDO-E subdimensions are highly correlated, as are the measures of environmentalism. On examination of the correlations between the SDO dimensions and environmentalism, it appears that SDO-E is the stronger correlate of all four aspects of environmentalism. 
Table 4.4.

Descriptive statistics, and correlation matrix between mean scores of each variable in Study $3 B$.

\begin{tabular}{|c|c|c|c|c|c|c|c|c|c|}
\hline & & Mean & SD & 1. & 2. & 3. & 4. & 5. & 6. \\
\hline 1. & SDO-D & $\begin{array}{c}2.87 \\
{[2.78,} \\
2.96]\end{array}$ & $\begin{array}{c}1.04 \\
{[.99,1.09]}\end{array}$ & $\begin{array}{c}\alpha \\
.83\end{array}$ & & & & & \\
\hline 2. & SDO-E & $\begin{array}{c}2.59 \\
{[2.50} \\
2.68]\end{array}$ & $\begin{array}{c}1.02 \\
{[.96,1.08]}\end{array}$ & $\begin{array}{c}.74 * * * \\
{[.69, .78]}\end{array}$ & $\begin{array}{c}\alpha \\
.85\end{array}$ & & & & \\
\hline 3. & Climate belief & $\begin{array}{c}6.21 \\
{[6.12,} \\
6.28]\end{array}$ & $\begin{array}{c}.93 \\
{[.83,1.04]}\end{array}$ & $\begin{array}{c}-.29 * * * \\
{[-.38,-} \\
.20]\end{array}$ & $\begin{array}{c}-.35 * * * \\
{[-.43,-} \\
.27]\end{array}$ & $\begin{array}{c}\alpha \\
.75\end{array}$ & & & \\
\hline 4. & Made sacrifice & $\begin{array}{c}4.41 \\
{[4.29} \\
4.53]\end{array}$ & $\begin{array}{l}1.44 \\
{[1.35,} \\
1.52]\end{array}$ & $\begin{array}{c}-.16 * * * \\
{[-.25,-} \\
.06]\end{array}$ & $\begin{array}{c}-.22 * * * \\
{[-.31,-} \\
.12]\end{array}$ & $\begin{array}{c}.27 * * * \\
{[.19, .36]}\end{array}$ & $\begin{array}{l}\alpha \\
.84\end{array}$ & & \\
\hline & $\begin{array}{l}\text { Willing to } \\
\text { sacrifice }\end{array}$ & $\begin{array}{c}5.24 \\
{[5.14,} \\
5.35]\end{array}$ & $\begin{array}{c}1.20 \\
{[1.11,} \\
1.30]\end{array}$ & $\begin{array}{c}-.28 * * * \\
{[-.36,-} \\
.19]\end{array}$ & $\begin{array}{c}-.39 * * * \\
{[-.47,-} \\
.30]\end{array}$ & $\begin{array}{c}.38 * * * \\
{[.28, .48]}\end{array}$ & $\begin{array}{c}.68 * * * \\
{[.61, .74]}\end{array}$ & $\begin{array}{l}\alpha \\
.86\end{array}$ & \\
\hline & $\begin{array}{l}\text { Value } \\
\text { environment }\end{array}$ & $\begin{array}{c}4.65 \\
{[4.50,} \\
4.80]\end{array}$ & $\begin{array}{c}1.67 \\
{[1.57} \\
1.77]\end{array}$ & $\begin{array}{c}-.15 * * \\
{[-.23,-} \\
.06]\end{array}$ & $\begin{array}{c}-.22 * * * \\
{[-.30,-} \\
.12]\end{array}$ & $\begin{array}{c}.28 * * * \\
{[.19, .38]}\end{array}$ & $\begin{array}{c}.56 * * * \\
{[.48, .62]}\end{array}$ & $\begin{array}{c}.64 * * * \\
{[.56, .69]}\end{array}$ & $\begin{array}{c}\alpha \\
\text { N/A }\end{array}$ \\
\hline
\end{tabular}

Note. Numbers in square brackets are $95 \%$ confidence intervals based on bootstrapping of 1000 iterations. SDO-D = dominance sub-dimension of SDO. SDO-E = anti-egalitarian sub-dimension of SDO. Along the diagonal are Cronbach's alphas for the scales. ${ }^{* *} p<.01, * * * p<.001$. 


\section{Path model}

The path model demonstrated adequate fit $\left(\chi^{2}(216)=474.55, p<.001, \chi^{2} / \mathrm{df}=\right.$ $2.20, \mathrm{CFI}=0.94, \mathrm{RMSEA}=0.05, \mathrm{SRMR}=0.05)$, and thus was accepted. As displayed in Figure 4.2, I replicated the pattern of findings from Study 3A, with SDOE a stronger correlate than SDO-D. In this dataset, I find that SDO-D does not relate to any of the environmental measure.

SDO-E is a significant predictor of willingness to make sacrifices for the environment $(\beta=-0.53, p<.001,95 \%$ CI $[-0.74,-0.32])$, endorsement of the value of protecting the environment $(\beta=-0.27, p=.007,95 \% \mathrm{CI}[-0.47,-0.08])$ and climate change belief $(\beta=-0.52, p<.001,95 \%$ CI $[-0.72,-0.32])$, and is a marginally significant predictor of having made sacrifices for the environment $(\beta=-0.20, p=$ $0.079,95 \%$ CI $[-0.42,0.02])$. Table 4.3 shows that SDO-E is a statistically stronger predictor than SDO-D for both willingness to make sacrifices and climate change belief. However, the difference in SDO-E and SDO-D's predictive ability is only marginally significant for environmental value, and not significant for having made sacrifices for the environment. 


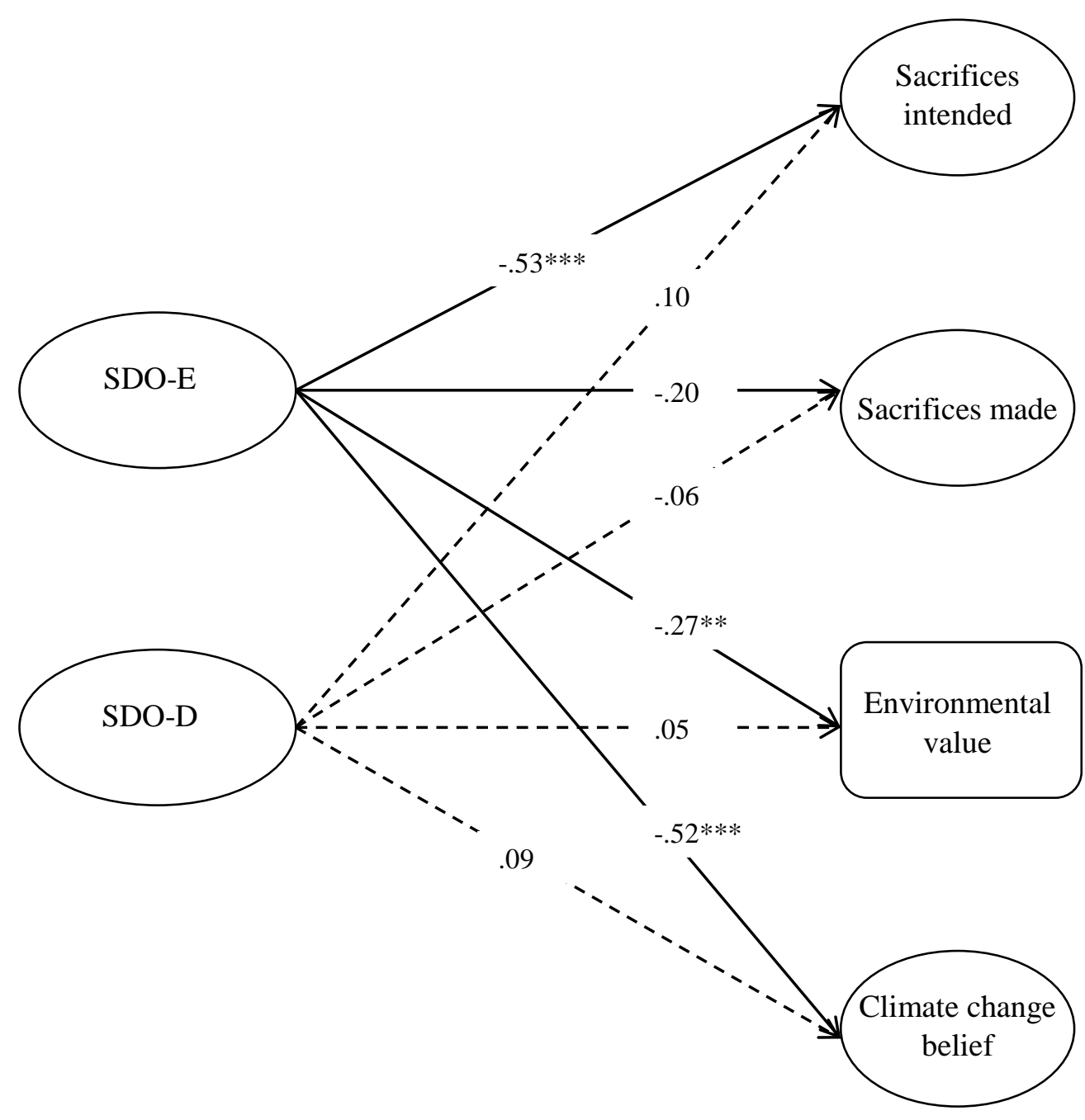

Figure 4.2. SDO-D (Dominance) and SDO-E (Egalitarianism) predicting environmental variables in Study 3B.

Note. Solid lines show significant paths $(* * p<.01, * * * p<.001)$ and dashed lines non-significant paths $(p>.01)$ 


\section{Comments}

In Study 3B, I again show that SDO-E is a superior predictor of environmentalism compared to SDO-D, adding weight to the argument that antiegalitarianism drives Social Dominants to dislike environmental action and deny climate change, more than the desire to dominate over nature.

One discrepancy between the studies is that SDO-D was a significant, albeit weaker, predictor of environmentalism in Study 3A, however was unrelated to environmentalism in Study 3B. With the dependent variables remaining consistent across these two studies, the difference in results could be due to the lower power in this study compared to Study 3A (smaller sample size, N of 504 versus 6516), or due to the difference in measurement $\left(\mathrm{SDO}_{7}\right.$ versus $\left.\mathrm{SDO}_{6}\right)$, or finally due to the difference in sample (students versus general population sample).

While the studies so far presented in this chapter converge to show that SDO$\mathrm{E}$ is the stronger predictor, they are based on cross-sectional data only. In Study 3C, I address this limitation by presenting the first study on how SDO-E and SDO-D work to predict environmentalism over time. 


\section{Study 3C}

\section{Longitudinal relation between the dimensions of SDO and environmentalism}

\section{Introduction}

In Study 3C, I aim to replicate and extend my earlier findings using a longitudinal dataset. I assess the relationship between the dimensions of SDO both within and across time points in the same dataset. Thus, as well as examining crosssectional links as in Studies 3A and 3B, I consider how SDO-D and SDO-E relate to environmentalism over time using a cross-lagged panel model. Thus far, I have shown SDO-E to be the superior predictor of environmentalism. Therefore, in this study I expect to replicate this finding.

\section{Method}

\section{Participants and procedure}

I include data from 674 first year psychology students at Victoria University of Wellington ( $77 \%$ female, mean age $=18.7$ years $)$. This is the same dataset as included in Study 2A, where I first tested the longitudinal association between environmentalism and SDO as a unitary construct.

In Study 2A, I found that SDO did not predict changes in environmentalism over time. However, this may have been the case because the model controlled for the effects of RWA, which (along with the stability coefficients) explained the majority of the variance in the environment-relevant variables, leaving little to be explained by SDO. Indeed, reanalysing the model from Study 2A when RWA is not controlled for, SDO does emerge as a significant longitudinal predictor of pro-environmental attitudes $(\beta=-.171, p=.002,95 \%$ CI $[-.281,-.062])$. Therefore, given that the model included here will not control for the effects of RWA, I believe it is worthwhile to test whether the dimensions will prove useful in predicting environmentalism longitudinally. 


\section{Measures}

For all measures, participants responded on a 7-point Likert scale from 1 (strongly disagree) to 7 (strongly agree).

Social dominance orientation. I used the same scale to measure SDO as in Study 3B: Ho et al.'s (2012) $\mathrm{SDO}_{7}$ scale.

Environmentalism. I used two environment-relevant variables in this study. This includes Dunlap and Van Liere's (1978) 12-item New Ecological Paradigm (NEP) scale to assess pro-environmental attitudes. To assess climate change denial, I used two related items (Time $1, r=0.64,95 \%$ CI [0.59, 0.70]; Time 2, $r=0.70,95 \%$ CI $[0.64,0.75])$, "Climate change is an entirely natural phenomenon - human action does not contribute importantly to it" and "Any changes in global climate simply reflects naturally occurring variation", that were combined to form a scale.

\section{Statistical analysis}

I follow an identical data-analytic procedure as in Study 3B for the confirmatory factor analyses (using Time 1 data) and cross-sectional path model. In the longitudinal model, all Time 1 variables are set to predict all Time 2 variables.

\section{Results}

\section{Confirmatory factor analyses}

Table 4.1 presents the results of my CFAs for Study 3C. Replicating both Ho et al. (2015) and findings from Study 3B, the four-factor model has better fit to the data than the unidimensional model of SDO ( scaled $\left.\Delta \chi^{2}(1)=58.84, p<.001\right)$.

\section{Descriptive statistics}

In Table 4.5, I present descriptive statistics, which replicate a similar pattern of correlations as observed in Studies 3A and 3B. Both within and across time points, SDO-E is a stronger correlate of pro-environmental attitudes, but scores on climate change denial appear to be comparable. 
Table 4.5 .

Descriptive statistics, and correlation matrix between mean scores of each variable in Study $3 C$.

\begin{tabular}{|c|c|c|c|c|c|c|c|c|c|c|c|}
\hline & & Mean & SD & 1. & 2. & 3. & 4. & 5. & 6. & 7. & 8. \\
\hline 1. & SDO-D T1 & $\begin{array}{c}2.99 \\
{[2.90,3.07]}\end{array}$ & $\begin{array}{c}1.04 \\
{[.99,1.09]}\end{array}$ & $\begin{array}{c}\alpha \\
.78\end{array}$ & & & & & & & \\
\hline 2. & SDO-E T1 & $\begin{array}{c}2.59 \\
{[2.51,2.67]}\end{array}$ & $\begin{array}{c}.99 \\
{[.93,1.04]}\end{array}$ & $\begin{array}{c}.71 * * * \\
{[.66, .75]}\end{array}$ & $\begin{array}{c}\alpha \\
.81\end{array}$ & & & & & & \\
\hline 3. & NEP T1 & $\begin{array}{c}5.24 \\
{[5.27,5.30]}\end{array}$ & $\begin{array}{c}.75 \\
{[.72, .79]}\end{array}$ & $\begin{array}{c}-.23 * * * \\
{[-.32,-.15]}\end{array}$ & $\begin{array}{c}-.29 * * * \\
{[-.38,-.20]}\end{array}$ & $\begin{array}{c}\alpha \\
.78\end{array}$ & & & & & \\
\hline 4. & Denial T1 & $\begin{array}{c}2.89 \\
{[2.78,3.01]}\end{array}$ & $\begin{array}{c}1.39 \\
{[1.30,1.46]}\end{array}$ & $\begin{array}{c}.25 * * * \\
{[.16, .33]}\end{array}$ & $\begin{array}{c}.24 * * * \\
{[.16, .32]}\end{array}$ & $\begin{array}{c}-.33 * * * \\
{[-.41,-.25]}\end{array}$ & - & & & & \\
\hline 5. & SDO-D T2 & $\begin{array}{c}3.06 \\
{[2.97,3.15]}\end{array}$ & $\begin{array}{c}1.08 \\
{[1.03,1.13]}\end{array}$ & $\begin{array}{c}.72 * * * \\
{[.67, .76]}\end{array}$ & $\begin{array}{c}.61 * * * \\
{[.56, .67]}\end{array}$ & $\begin{array}{c}-.28 * * * \\
{[-.36,-.20]}\end{array}$ & $\begin{array}{l}.27 * * * \\
{[.19, .34]}\end{array}$ & $\begin{array}{c}\alpha \\
.83\end{array}$ & & & \\
\hline 6. & SDO-E T2 & $\begin{array}{c}2.71 \\
{[2.63,2.80]}\end{array}$ & $\begin{array}{c}1.04 \\
{[.99,1.09]}\end{array}$ & $\begin{array}{c}.59 * * * \\
{[.52, .64]}\end{array}$ & $\begin{array}{c}.63 * * * \\
{[.57, .68]}\end{array}$ & $\begin{array}{c}-.27 * * * \\
{[-.35,-.18]}\end{array}$ & $\begin{array}{c}.27 * * * \\
{[.18, .36]}\end{array}$ & $\begin{array}{c}.76 * * * \\
{[.73, .80]}\end{array}$ & $\begin{array}{c}\alpha \\
.86\end{array}$ & & \\
\hline 7. & NEP T2 & $\begin{array}{c}5.27 \\
{[5.20,5.34]}\end{array}$ & $\begin{array}{c}.86 \\
{[.83, .90]}\end{array}$ & $\begin{array}{c}-.27 * * * \\
{[-.35,-.19]}\end{array}$ & $\begin{array}{c}-.31 * * * \\
{[-.38,-.23]}\end{array}$ & $\begin{array}{c}.71 * * * \\
{[.66, .75]}\end{array}$ & $\begin{array}{c}-.32 * * * \\
{[-.40,-.25]}\end{array}$ & $\begin{array}{c}-.39 * * * \\
{[-.46,-.32]}\end{array}$ & $\begin{array}{c}-.38 * * * \\
{[-.46,-.29]}\end{array}$ & $\begin{array}{c}\alpha \\
.85\end{array}$ & \\
\hline 8. & Denial T2 & $\begin{array}{c}2.76 \\
{[2.65,2.87]} \\
\end{array}$ & $\begin{array}{c}1.36 \\
{[1.28,1.44]} \\
\end{array}$ & $\begin{array}{c}.24 * * * \\
{[.16, .32]} \\
\end{array}$ & $\begin{array}{c}.22 * * * \\
{[.13, .30]} \\
\end{array}$ & $\begin{array}{c}-.35 * * * \\
{[-.43,-.28]} \\
\end{array}$ & $\begin{array}{c}.58 * * * \\
{[.50, .65]} \\
\end{array}$ & $\begin{array}{c}.37 * * * \\
{[.29, .44]} \\
\end{array}$ & $\begin{array}{c}.37 * * * \\
{[.29, .45]} \\
\end{array}$ & $\begin{array}{c}-.47 * * * \\
{[-.53,-.41]} \\
\end{array}$ & - \\
\hline
\end{tabular}

Note. Numbers in square brackets are 95\% confidence intervals based on bootstrapping of 1000 iterations. SDO-D = dominance sub-dimension of SDO. SDO-E $=$ anti-egalitarian sub-dimension of SDO. NEP = new environmental paradigm. Along the diagonal are Cronbach's alphas for the scales. ${ }^{* *} p<.01$, $* * * p<.001$. 


\section{Cross-sectional path model}

In the cross-sectional models, I set SDO-D and SDO-E to predict scores on the NEP and climate change denial in two separate models: one for each time point. Both models demonstrated adequate fit to the data (Time $1: \chi^{2}(399)=1154.38, p<.001$, $\chi^{2} / d f=2.89 ; \mathrm{CFI}=.84, \mathrm{RMSEA}=.05, \mathrm{SRMR}=.06$; Time $2: \chi^{2}(399)=1413.91, p<$ $\left..001, \chi^{2} / d f=3.54, \mathrm{CFI}=.84, \mathrm{RMSEA}=.06, \mathrm{SRMR}=.07\right)$.

These models are presented in Figures 4.3 (Time 1) and 4.4 (Time 2). Overall, the results are consistent at both time points. Each dimension of SDO exhibits positive associations with denial of climate change and negative relationships with pro-environmental attitudes. Furthermore, SDO-E remains the stronger predictor of environmentalism, predicting pro-environmental attitudes at both time points (Time 1: $\beta=-.54, p<.001,95 \%$ CI $[-.77,-.30]$; Time $2: \beta=-.42, p=.001,95 \%$ CI $[-.71,-.17])$ and climate change denial at time two only (Time $1: \beta=.12, p=.354,95 \%$ CI [-.14, $.38]$; Time $2: \beta=.26, p=.027,95 \%$ CI $[.04, .73])$. SDO-D did not predict proenvironmental attitudes (Time $1: \beta=.18, p=.130,95 \%$ CI [-.05, .42]; Time 2: $\beta=-$ $.04, p=.737,95 \%$ CI $[-.23, .16]$ ) or climate change denial (Time $1: \beta=.22, p=.08$, 95\% CI $[-.03, .47]$; Time $2: \beta=.19, p=.11,95 \% \mathrm{CI}[.05, .48])$ at either time point.

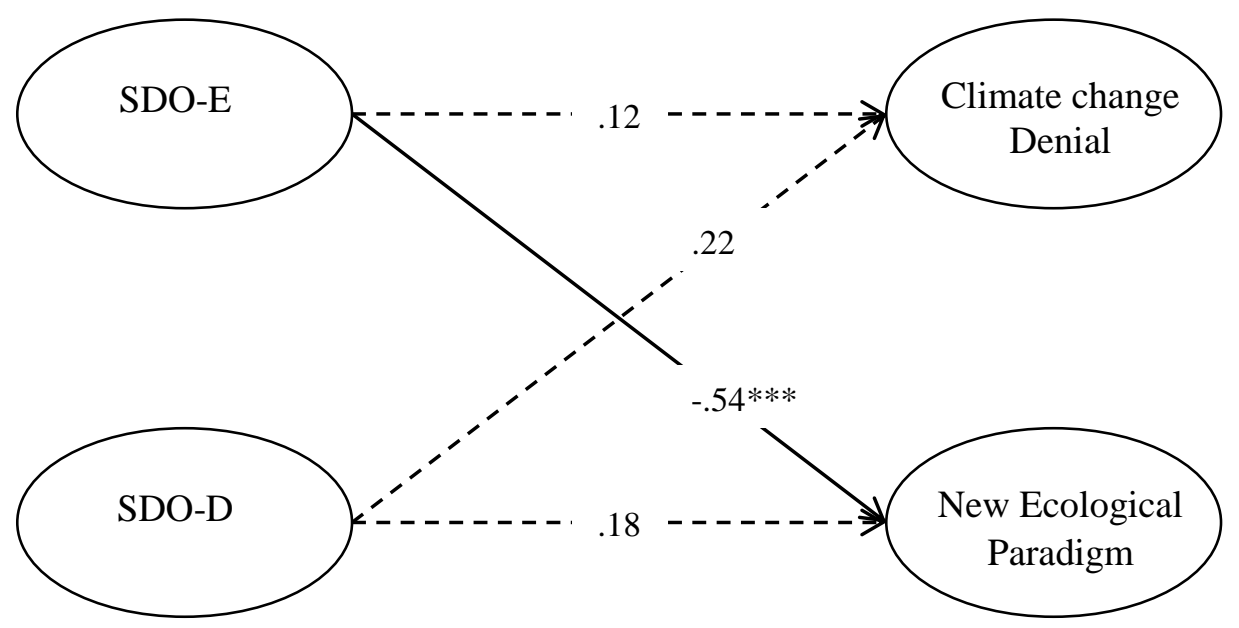

Figure 4.3. Time one SDO-D (Dominance) and SDO-E (Egalitarianism) predicting environmental variables concurrently in Study 3C.

Note. Solid lines show significant paths $(* p<.05, * * * p<.001)$ and dashed lines nonsignificant paths $(p>.05)$. 


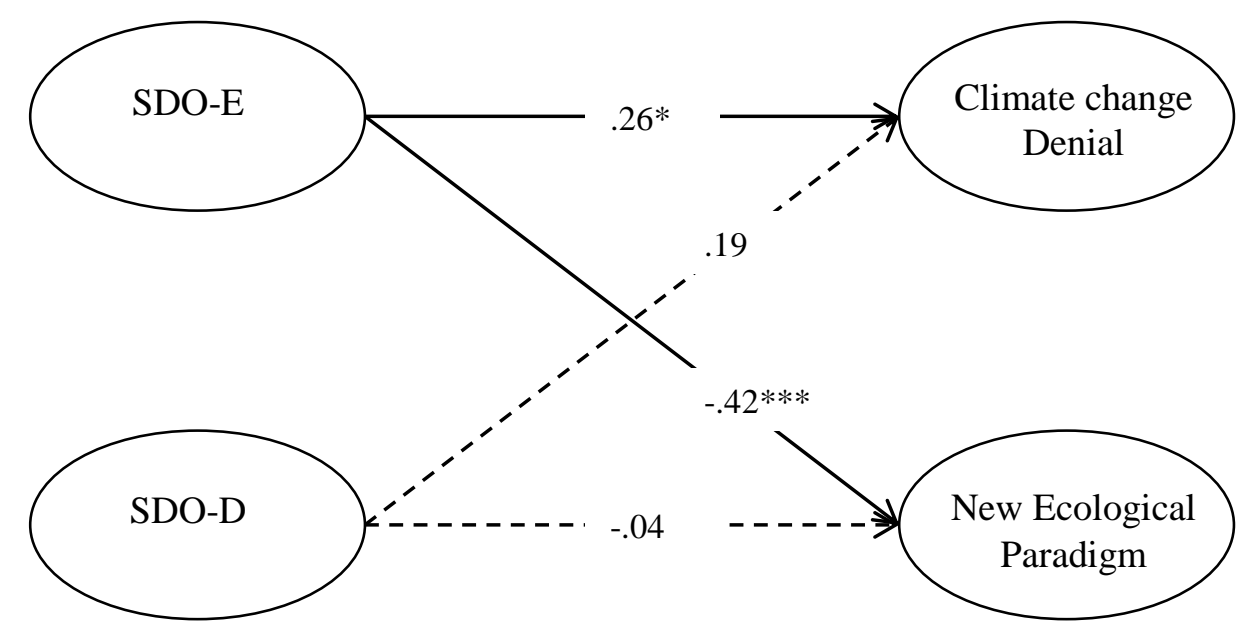

Figure 4.4. Time two SDO-D (Dominance) and SDO-E (Egalitarianism) predicting environmental variables concurrently in Study $3 \mathrm{C}$.

Note. Solid lines show significant paths $(* p<.05, * * * p<.001)$ and dashed lines nonsignificant paths $(p>.05)$.

\section{Longitudinal path model}

The cross-lagged panel model, as shown in Figure 4.5, again evidenced good fit to the data $\left(\chi^{2}(1652)=3445.55, p<.001, \chi^{2} / \mathrm{df}=2.09 ; \mathrm{CFI}=0.87 ; \mathrm{RMSEA}=\right.$ 0.04; SRMR =0.06). I firstly note that SDO-D and the environmental variables largely remain stable over time (SDO-D: $\beta=0.85, p<.001,95 \%$ CI $[0.69,1.01]$; Denial: $\beta=0.60, p<.001,95 \%$ CI [0.50, 0.69]; NEP: $\beta=0.73, p<.001,95 \%$ CI $[0.65,0.81])$, while SDO-E is comparatively less stable over time $(\beta=0.32, p<.001$, $95 \%$ CI $[0.15,0.50])$. However, rather than this indicating that people are less likely to retain their levels of SDO-E over time, it appears that the weak autoregressive path is attributable to SDO-E's shared variance with SDO-D This is because when SDO-D is not controlled for, the association between SDO-E at Time 1 and Time 2 is strong $\beta$ $=0.60, p<.001,95 \%$ CI $[0.53,0.68])$. Indeed, Figure 5 shows that SDO-D predicts an increase in SDO-E over time $(\beta=0.34, p<.001,95 \%$ CI [0.17, 0.51]), while SDO-E is not a significant predictor in changes in SDO-D over time $(\beta=-0.10, p=$ $.269,95 \%$ CI $[-0.27,0.08])$. 
My main prediction for this model was that SDO-E would prospectively predict changes in environmentalism. However, on examination of the relevant crosslagged paths, it appears that neither dimension of SDO significantly predicts changes environmentalism over time. Instead, environmentalism predicts SDO over time.

Endorsement of climate change denial at Time 1 related to increased endorsement of SDO-E at a later point in time $(\beta=0.09, p=.039,95 \%$ CI $[0.01,0.17])$, while higher levels of pro-environmental attitudes were related to decreased endorsement of SDOD five months later $(\beta=-0.10, p=0.017,95 \%$ CI $[-0.18,-0.02])$. 
TIME ONE

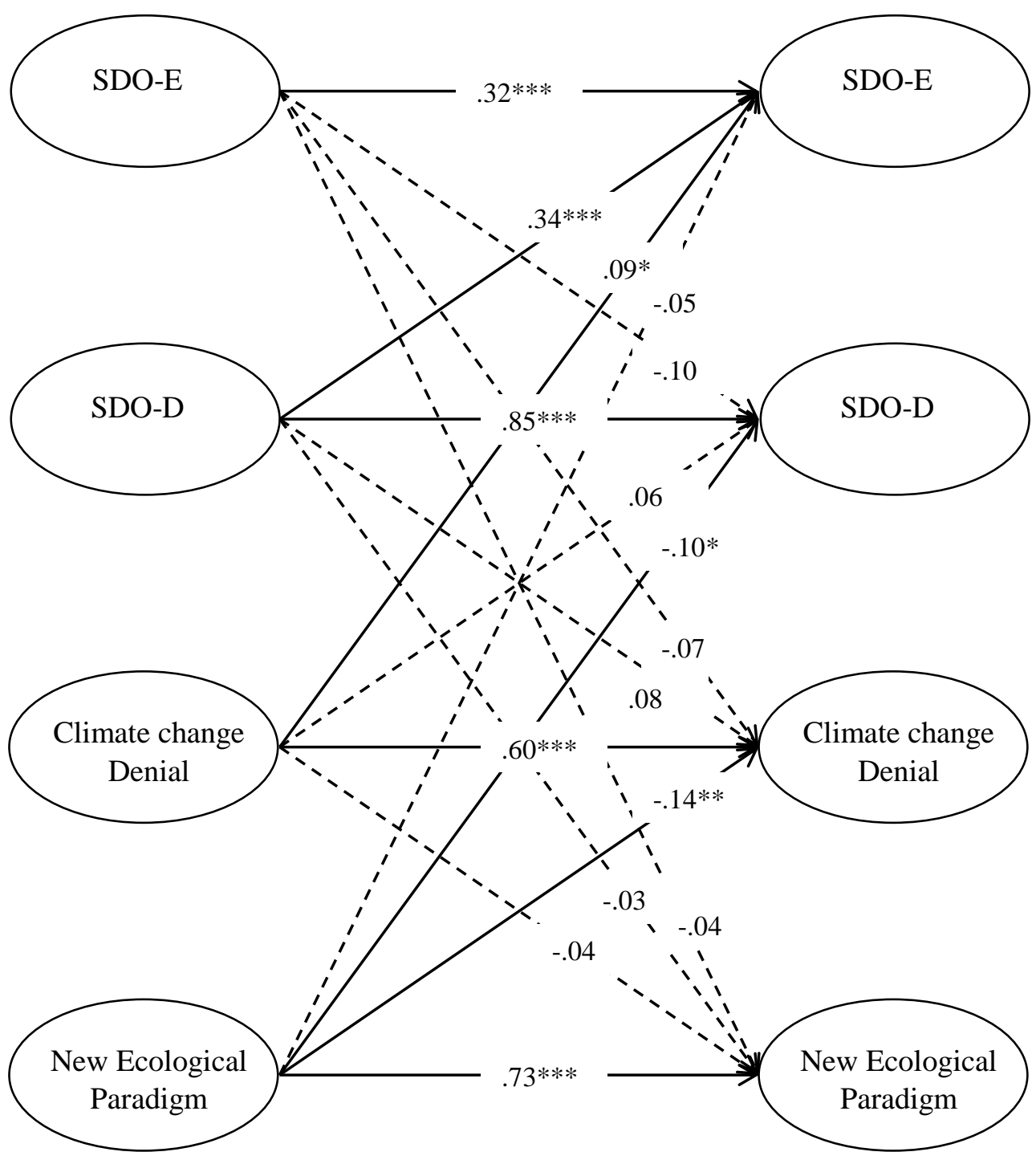

Figure 4.5. Longitudinal associations between SDO-D (Dominance) and SDO-E (Egalitarianism) and environmentalism in Study 3C.

Note. Solid lines show significant paths $(* p<.05, * * p<.01, * * * p<.001)$ and dashed lines non-significant paths $(p>.05)$ 
One potential explanation for the lack of longitudinal association between the SDO dimensions and environmentalism is that the majority of the variance was being accounted for by the stability coefficients. I ran several separate path models to test whether this would explain the findings. Firstly, I entered SDO-D and SDO-E as measured at Time 1 as predictors of Time 2 environmental variables. In this model, the measures of environmentalism are not residualized and therefore instead of examining change in climate change denial and pro-environmental attitudes, I am simply examining the relationship over time. I expected that, given there is more variance to be explained in the environmental variables now that the stability paths are removed, SDO-E at least would predict environmentalism. The model yielded acceptable fit $\left(\chi^{2}(399)=1454.70, p<.001, \chi^{2} / d f=3.65 ; \mathrm{CFI}=.85 ; \mathrm{RMSEA}=.06\right.$; SRMR = .06). Depicted in Figure 4.6, I found that initial levels of SDO-E were indeed predictive of later scores on the NEP $(\beta=-.43, p<.001,95 \%$ CI [-.62, -.23]), thus partially supporting this prediction, but all other associations were not significant.

Time 1

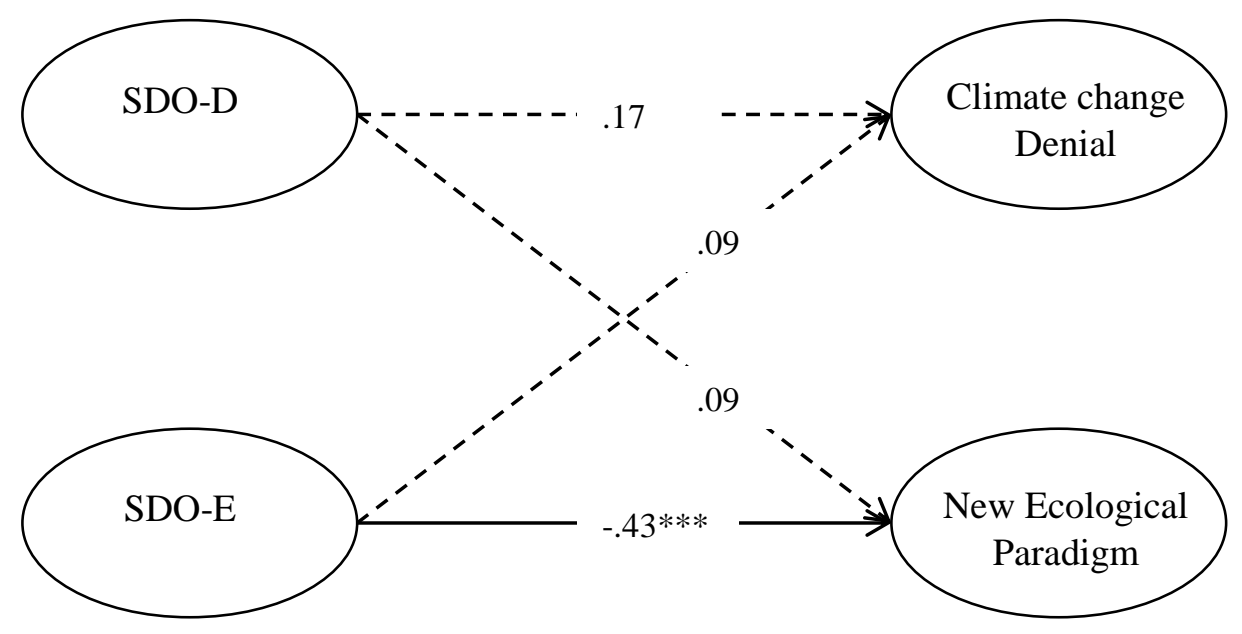

Figure 4.6. SDO-D (Dominance) and SDO-E (Egalitarianism) at Time 1 predicting environmentalism at Time 2 in Study 3C.

Note. Solid lines show significant paths $(* * * p<.001)$ and dashed lines nonsignificant paths $(p>.05)$. 
While the non-residualized model did show one longitudinal association with SDO-E, it is pertinent to test the reverse direction, from environmentalism to SDO, which did yield some significant paths even in the fully saturated model. In this model, NEP and climate change denial at Time 1 are set to predict SDO-D and SDO$\mathrm{E}$ at Time 2, which formed a well-fitting model $\left(\chi^{2}(399)=1524.91, p<.001, \chi^{2} / \mathrm{df}=\right.$ $3.82 ; \mathrm{CFI}=.84 ; \mathrm{RMSEA}=.07 ; \mathrm{SRMR}=.06)$. In this model, graphed in Figure 4.7, denial of climate change was positively related to subsequent SDO-D $(\beta=.26, p<$ $.001,95 \% \mathrm{CI}[.16, .36])$ and SDO-E $(\beta=.24, p<.001,95 \% \mathrm{CI}[.14, .33])$, and scores on the NEP similarly predicted both SDO-D $(\beta=-.20, p<.001,95 \%$ CI $[-.30,-.10])$ and SDO-E $(\beta=-.20, p<.001,95 \%$ CI $[-.30,-.11])$.

Time 1

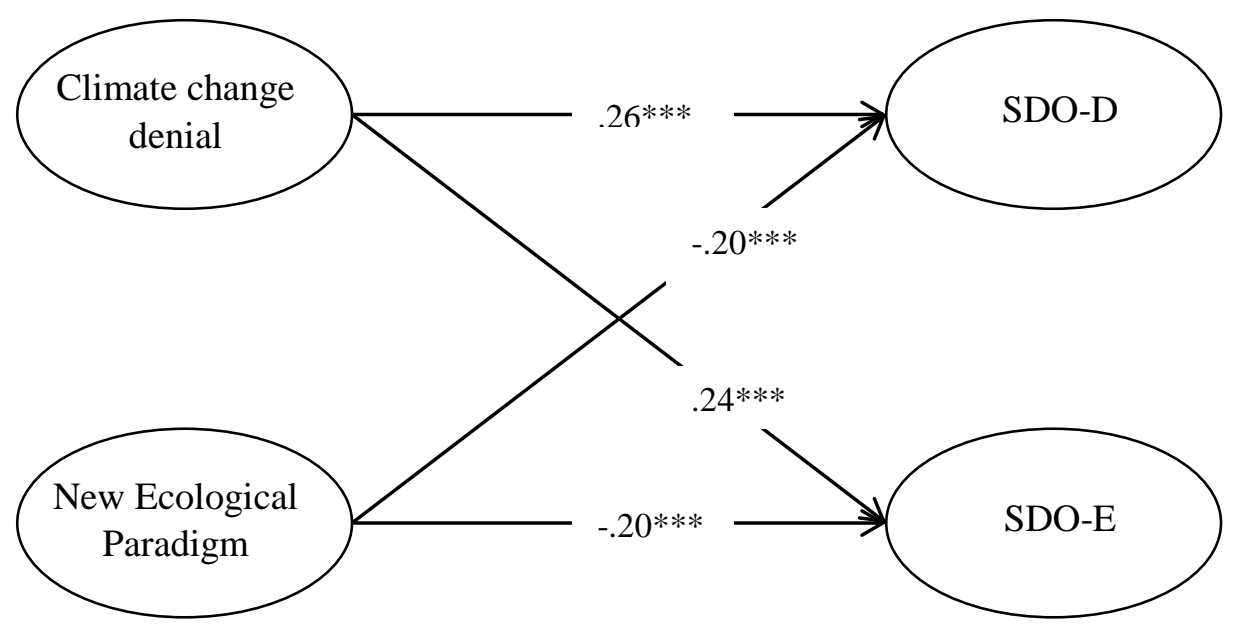

Figure 4.7. Environmental variables at Time 1 predicting SDO-D (Dominance) and SDO-E (Egalitarianism) at Time 2 in Study 3C.

Note. Solid lines show significant paths $(* * * p<.001)$ and dashed lines nonsignificant paths $(p>.05)$.

Therefore, the results of the additional path analyses confirm the findings of the main model: the direction of the association between the dimensions of SDO and environmentalism appear to go in the direction of environmental attitudes to ideology. 


\section{Comments}

Study 3C again supported the four-factor model of SDO (crossing direction of wording with Dominance/Egalitarianism facets), and provided support for SDO-E as the strongest cross-sectional predictor of environmentalism. At both time points, SDO-E predicted concurrent scores on pro-environmental attitudes, and SDO-E was a significant predictor of climate change denial at Time 2. Meanwhile, SDO-D did not predict either environmental variable, at either point in time.

The NEP scale, which I used to measure pro-environmental attitudes, is made up of several items tapping human dominance over nature. For example, the items "Humans have a right to modify the natural environment to suit their needs" and "Mankind was created to rule over the rest of nature" both present humans as dominant and nature as subordinate. Therefore, it is surprising that when environmentalism is indexed using this measure, there is no evidence that SDO-D is a predictor. However, the measure does include items hinting at equality between humans and non-human animals, which are likely opposed by those endorsing SDO-E (e.g. "Plants and animals have as much right as humans to exist").

The novel component of Study 3C was the longitudinal examination of these relationships. While I expected SDO-E to come out on top as the stronger predictor, instead I found that it is environmental attitudes that drive changes in ideology over time. This finding is explored further below in the Discussion of all studies presented in Chapter 4. 


\section{Discussion of Studies 3A, 3B \& 3C}

While a growing number of recent studies have highlighted the importance of SDO in relation to environmental attitudes and climate change belief (e.g. Milfont et al., 2013), the recent confirmation of a two-dimensional view of SDO has largely been neglected within environmental psychology. Ho et al. $(2012,2015)$ demonstrated the utility of separating SDO into SDO-D and SDO-E, to reflect a focus on dominance over other social groups, and opposition to equality, respectively. They show SDO-D to relate to overt oppression, whilst those endorsing SDO-E prefer more subtle means of oppression achieved by opposing policies aimed at equalizing conditions between groups.

In Chapter 4, I test the utility of the two-dimensional model of SDO in relation to environmentalism in three New Zealand samples. My first main finding was the confirmation of SDO as reliably forming two separate domains, regardless of which measure of SDO was used. The fit improved even more when the direction of wording (pro- or con-trait) was taken into consideration as well as the content (SDOD and SDO-E).

Next, extending the two-dimensional view of SDO into the environmental domain, I show that the two dimensions do indeed differentially relate to environmentalism. Across three studies, I note that SDO-E is the strongest (and in some cases, the only) significant predictor of environment-relevant variables. However, the pattern of associations holds only in cross-sectional datasets, with the reverse direction significant over time: environmentalism predicts endorsement in the SDO dimensions. In this discussion, I review the main findings presented in Chapter 4.

\section{Factor structure of SDO scales}

I consistently show that the two-factor solution to SDO provides better fit to the data than the unidimensional structure originally defended by Sidanius and Pratto (1993). When SDO is indexed by scores on the $\mathrm{SDO}_{7}$ scale, the four-factor solution is best, which takes into account both wording and content factors. Ho et al. (2012, 2015) first validated the $\mathrm{SDO}_{7}$, including confirming its composition as two separate subdimensions. I provide additional evidence for its psychometric properties throughout this chapter by replicating Ho et al.'s (2015) findings to show that 
accounting for both the content (i.e. SDO-D and SDO-E) components of the scale, as well as the wording direction (pro- and con-trait) provides the best fit to the data. Furthermore, I achieve similar Cronbach's alpha values as Ho et al. (2015), which exceed the threshold to indicate internal reliability (e.g. Nunnally, 1978).

By Ho et al.'s (2015) recommendation, researchers ought to abandon the $\mathrm{SDO}_{6}$ and opt for the new $\mathrm{SDO}_{7}$ instead. This makes intuitive sense, given the superior psychometric properties of the $\mathrm{SDO}_{7}$ and the ability to adequately measure the dimensions of SDO separately. However, note that the findings of Study 3A, which relied on data collected using the $\mathrm{SDO}_{6}$ scale, closely align with the results of our other two studies, which both employ the $\mathrm{SDO}_{7}$. The two-factor model of SDO provided good fit to the data, and although the Cronbach's alpha values were lower, this is not unexpected given that the shortened scale used just three items to measure each dimension of SDO. Furthermore, findings of all three studies converged to show SDO-E as the stronger predictor, regardless of the measure used. Therefore, although the $\mathrm{SDO}_{6}$ has the problem of conflating wording direction and content of its items, this does not appear to interfere with the reliability and utility of the measure. Consequently, I argue that existing data that measured SDO using this and older scales are not necessarily flawed and should certainly not be rejected. This is especially the case if SDO is to be used as a unidimensional scale, as Studies 1A and 1B demonstrated no difference in the strength of the mean correlations between SDO and environmentalism based on scale type.

As one final point, Ho et al. (2015) explain that space constraints sometimes push researchers to use shortened versions of scales. They show that a short, 8 -item version of the $\mathrm{SDO}_{7}$ is also valid (four items to measure each dimension). Using the data from Studies 3A and 3B, I took the same subset of items as Ho et al. to replicate the one- and two-dimensional CFAs using shortened measures. These results are presented in Appendix $\mathrm{C}$, and show that the best fitting models are again the ones that take method and content factors into account (four-factor models). Consistent with theory, the two-factor models provided significantly better fit to the data than the onefactor model (Study 3B: $\Delta \chi^{2}(1)=17.86, p<.001$; Study 3C: $\Delta \chi^{2}(1)=6.76, p=.009$ ). These findings confirm that the two-dimensional approach to SDO can be successfully applied in large-scale surveys, where space constraints may demand that fewer items be included. 


\section{The relation between the dimensions of SDO and environmentalism}

As discussed in the General Introduction, Sidanius and Pratto's (1993, 1999) Social Dominance Theory (SDT) was developed with a focus on understanding intergroup relations. SDO is therefore unsurprisingly more strongly related to aspects of intergroup attitudes than to environmental attitudes. For instance, Sibley and Duckitt (2008) demonstrate that SDO exhibits a strong meta-analytic correlation $(r=$ .55) with prejudice, while the overall correlation with environmentalism is moderate $(r=-.29 ;$ Study 1A). However, it is interesting that SDO relates to environmentalism at all. The implication of the SDO-environmentalism link is that the SDT can be broadened to include environmental issues, which are not explicitly tied to intergroup relations. However, by examining how the dimensions of SDO are related to environmentalism, I advance understanding of what aspect underlies this association.

Consistent with an SDO-E interpretation of this relationship, individuals who endorse SDO might use the environment to secure the dominant group's place at the top of the hierarchy. In contrast, perhaps to a lesser extent (if at all), their valuing of dominance over other social groups (i.e. SDO-D) may extend to support human dominance over nature. I show that individuals who show relatively greater opposition to group equality (i.e., greater endorsement of SDO-E) are more likely to deny climate change, be unwilling to make personal sacrifices for the cause, and do not consider protecting the environment an important principle to live by.

At face value, endorsement of SDO-D might seem like a more threatening attitude to hold, as it has been linked to overt aggression, violent subjugation of low status groups, and outward prejudice (Ho et al., 2015). However my research suggests that endorsement of SDO-D might be less threatening in the environmental context. Instead, it is the 'quieter' high-SDO-E individuals who ensure inequality is maintained through more subtle routes, who are less concerned about, and dedicated to, environmental issues, thus potentially leading to worse outcomes for the environment. The preference for dominance (as in SDO-D) is instead either minimally or not at all related to anti-environmentalism.

My findings align with the theoretical position of Milfont and Sibley (2014) as described in their hierarchy enforcement hypothesis of environmental exploitation. According to the hypothesis, the relationship between SDO and environmentalism 
exists because Social Dominants choose to exploit the environment in order to secure or strengthen the superior status of the dominant group. This is achieved, as they demonstrated, by distributing natural resources in a way that favours the dominant group (see also Jackson et al., 2013, Study 2). This finding is reminiscent of Ho et al.'s (2015) characterisation of endorsers of SDO-E, who they state "prefer hierarchies where resources are inequitably distributed, and which can be defended by anti-egalitarian ideologies" (p. 1022).

Jackson et al. (2013, Study 4) noted that high-SDO's are content sending an environmental hazard offshore, providing their ingroup benefits and a lower status outgroup is left with the consequences. Though they do not directly measure SDO-D in this study, the decisions of Socially Dominant participants in their study do seem consistent with the dominance dimension of SDO. Future research ought to consider the possibility that SDO-D may indeed be a strong(er) predictor of environmentalism depending on how this is operationalised. I did consider this possibility and included a range of environment-relevant variables in this Chapter - and found SDO-E to consistently relate more strongly (though not always significantly so) than SDO-D. However, it is possible that still other aspects of environmentalism may relate more strongly to SDO-D.

\section{Longitudinal findings}

In Study 3C, I first replicated the pattern of cross-sectional findings as reported in Studies 3A and 3B, again demonstrating SDO-E to be the stronger crosssectional predictor. I extended on this to test the potential across-time effects of the dimensions of SDO on environmentalism. However, I found that neither dimension of SDO predicted changes in environmentalism over time. Instead, the environmental variables differentially predicted changes in SDO, with climate change denial predictive of increases in SDO-E, while pro-environmental attitudes predicted decreases in SDO-D over a five-month period. The unidirectional nature of these findings imply that denial of climate change precedes an increase in endorsement of anti-egalitarianism, while preferences for harmonious relations between humans and nature precede a decrease in dominance beliefs. Put simply: environmentalism is driving ideology. 
This pattern of results was originally reported in Study 2A, where although SDO did not predict environmentalism longitudinally, climate change denial predicted a small increase in endorsement of SDO. In Study 2B, I showed that SDO predicted a decreased level of support for making sacrifices for the environment, and there was a small reciprocal effect of this index of environmentalism on SDO. Here, I delve into the possible reasons for this directional association.

Based on both theoretical and empirical grounds, I expected SDO to precede environmental attitudes. However, both theory and existing longitudinal research mostly comes from research on intergroup relations. For instance, Duckitt's (2001) dual process model suggests that viewing the world as a competitive jungle gives way to endorsement of SDO (hence, a causal relationship), and this in turn promotes prejudice towards subjugated outgroups (another causal path). This is because seeing the world as competitive motivates high-SDO's to ensure the dominant group maintains its place at the top by collecting a disproportionate amount of resources and social power. This directional prediction is supported by some empirical evidence (e.g. Asbrock et al., 2010). However, while Kteily, Sidanius and Levin (2011) replicated the lagged effect of SDO on prejudice, they also show that prejudicial attitudes precede SDO, thus implying a bidirectional association between ideology and attitudes.

Potentially explaining the lack of association from ideology to environmentalism over time, Sidanius and Pratto (1999) note that for those in hierarchy-attenuating contexts, SDO does not necessarily precede attitudes. Students studying psychology fall into this category (e.g. Jetten \& Iyer, 2010). Instead, their attitudes may be more predictive of ideology because attitudes direct thoughts, feelings and reasoning, which inform moral decision making, rather than the other way around (Hatemi \& McDermott, 2016). Therefore, the directional relationship found in Study 3C does fit with some theoretical models, and with findings that question a simple unidirectional role for SDO in determining attitudes (e.g. Kteily et al., 2011; Turner \& Reynolds, 2003), especially for my student sample (see Chapter 2, and Jetten \& Iyer, 2010). However, as the longitudinal association is based on such limited findings in the environmental domain, future research must further investigate the longitudinal nature of the SDO-environmentalism link. This is especially the case 
given the limitations of the Study 3C data (student sample, spanning only five months).

The longitudinal investigation also yielded interesting information about the stability of the SDO dimensions and environmentalism. Specifically, I found that people tend to retain their levels of SDO-D over time, whilst endorsement of SDO-E appeared less stable. Sidanius, Levin, Van Laar and Sears (2008) showed that the unidimensional SDO scores are exceptionally stable over time, with a correlation between first measurement and measurement one year later exceeding .80, and testretest reliability remaining high even after five years $(r=.74)$. In my sample, the SDO-D dimension experienced little change over time, both when stability is captured by the correlation between endorsement of SDO-D at Time 1 and Time 2, and by the auto-regressive path in the main model. However, only one such index conveyed the stability of SDO-E over time: the across time correlation, whilst the longitudinal path model implied that SDO-E is less stable compared to SDO-D. However, I argue that this is explained by the association between SDO-E and SDO-D, with SDO-D explaining more variance in SDO-E across time and hence weakening its own autoregressive association.

\section{Conclusion}

Across three studies, I confirm that SDO reliably separates into two dimensions, and show that cross-sectionally, SDO-E is consistently the stronger predictor of environment-relevant variables than SDO-D. I am confident in this finding, as it was replicated across samples (general population and student), scales $\left(\mathrm{SDO}_{6}\right.$ and $\mathrm{SDO}_{7}$ ), and when utilizing different indices of environmentalism (climate change denial, pro-environmental attitudes and intentions, and valuing the environment).

This research identifies endorsers of SDO-E as key targets for climate change communications, as they are more of a threat to the environment than those endorsing SDO-D. It also highlights the need to avoid framing environmental action as encouraging intergroup equality, so that these actions are more palatable to those endorsing SDO-E. Finally, from the longitudinal findings in Study 3C, I suggest researchers should consider the possibility that environmental attitudes may influence 
later endorsement of SDO, rather than assuming the direction of the association flows from ideology to attitudes. 


\section{Chapter 5}

\section{Examining the discourses of high- and low-Social Dominants surrounding environmental issues}

\section{Study 4}

I began my thesis by demonstrating that SDO and RWA are each key correlates of attitudes towards the environment and climate change (Study 1A \& 1B). I also showed that this ideology-environmentalism link holds across time, with both SDO and RWA prospectively predicting attitudes towards making sacrifices for the environment in the general population (Study 2B), though only RWA predicted a significant increase in climate change denial over time among students (Study 2A).

By necessity (and reflecting a longer track record of research on the topic) I have considered RWA as a part of the puzzle of environmental attitudes and actions, but the main focus of this thesis is to clarify the nature of the relationship between SDO and environmental attitudes: how does a preference for dominance in an intergroup context extend to the environmental sphere? To supplement my initial studies, I then narrowed in on the specific aspect of SDO that more clearly underlies the association, demonstrating that anti-egalitarian attitudes (i.e. those favouring inequality) more strongly predict attitudes towards the environment than dominance (favouring intergroup control; Study 3A, 3B, \& 3C).

Each of the studies I have presented thus far are quantitative in nature, meaning they rely on quantifying responses to survey items, and show a statistical relationship between responses. In fact, to my knowledge, all research to date in this area relies on quantitative methods. While findings from this body of research are

fairly consistent (e.g. see Chapter 2), the knowledge gained from quantitative work is limited.

Participants engaging with quantitative research are typically restricted to answering only the set of items or statements chosen by the researcher, indicating 
their response by selecting from a set range of answer options (e.g. rating their agreement to prescribed statements numerically). Furthermore, they are given no opportunity to justify or expand on their responses. Qualitative research takes a more exploratory, and less pre-defined approach, by asking open-ended questions that allow greater freedom and scope in the response (Biggerstaff, 2012). Qualitative methods may, therefore, encourage participants to give rich responses and facilitate a deeper understanding of an issue (Queirós, Faria \& Almeida, 2017; Rahman, 2017). In this chapter, I explore how qualitative methods may generate new information on the SDO-environmentalism link that is not as easily accessed via survey-based approaches.

Several researchers have utilized qualitative methods to understand attitudes on the environment and climate change. For instance, Stoll-Kleeman, O'Riordan, and Jaeger (2001) conducted a series of focus groups with individuals asked to think about what life might be like 30 years into the future if energy use continued in a businessas-usual fashion, versus a future where energy consumption is severely curtailed. Many participants associated the high-energy use scenario with death and catastrophe. However, despite this concern for the consequences of climate change, participants also expressed concern relating to mitigating action itself, constructing barriers to action, deflecting responsibility for acting onto others, and citing feelings of powerlessness. Lorenzoni, Nicholson-Cole and Whitmarsh (2007) similarly found evidence of the barriers to action on climate change, finding that these exist at both the individual level (e.g. insufficient knowledge, fatalism) and societal level (e.g. businesses/govenrment inaction, social norms), mirroring the individual and external barriers identified in earlier work (e.g. Stern, 2002). Both of these articles frame barriers as tools to excuse, justify, and alleviate guilt over inaction on climate change.

To my knowledge, no researchers to date have recruited interviewees based on their endorsement of SDO to examine how Social Dominants discuss their attitudes on environmental issues. In this chapter, I work to explore the discourses of individuals with varying levels of SDO who were asked to talk about environmental issues. The corpus of data I analyse in Study 4 are from 59 semi-structured interviews with individuals endorsing low, moderate, and high levels of SDO. 
Participants were asked for their opinions on climate change, whether they perceive it to be a problem, and to think about potential solutions and barriers to solving the issue. I analyse these discussions using thematic analysis, which is a framework of analyzing qualitative responses to look for patterns and common themes in the data (Braun \& Clark, 2006). The goal of this work is to capture different ways that people talk about the environment and climate change, including the solutions they see as feasible for this problem.

Given that the researcher plays an active role in qualitative research (Braun \& Clarke, 2013), I made few predictions prior to analysis. This was to avoid (to the extent possible) my preconceptions influencing my approach to the data, and because my aim was exploratory. However, given the previous work in this area (e.g. Lorenzoni et al., 2007; Stoll-Kleeman et al., 2001), and the questions I use to guide the interviews (see Method section below), I anticipate that the ideas discussed in the interviews will center around the solutions and barriers of climate change.

\section{Method}

Data collection for this study was comprised of two phases. In Phase 1, I conducted an online survey measuring levels of SDO within a large student sample. In Phase 2, a subset of these participants (selected based on their SDO level) were invited to participate in an additional study, where they would be asked to talk about their ideas about social and political issues in depth (i.e., the interview study). I relied on data from student samples for this research as, despite appearing towards the end of the thesis, data for this study were collected at the start of my $\mathrm{PhD}$, and therefore $\mathrm{I}$ did not yet know that the SDO-environmentalism association differed by sample type. In light of the findings presented in Chapter 2, this research should have more sensibly been conducted with a general population sample. The Victoria University of Wellington Human Ethics Committee approved each stage of this study (Ethics ID number: 0000022835).

\section{Participants}

Phase 1. In order to recruit participants low, moderate, and high in SDO, I first conducted a larger-scale online survey measuring endorsement of SDO. In this phase, 504 introductory psychology students at Victoria University of Wellington signed up through their online introductory psychology research participation 
program (IPRP), earning partial credit towards their required participation hours for completing the survey. They then participated in the online survey hosted by SurveyMonkey (www.surveymonkey.com).

At the end of the survey, participants were asked if they were willing to be contacted about participating in a related study that explores the ideas in the questionnaire further. If they agreed, I recorded their student ID number, which was stored so that those who met our selection criteria (i.e. equal numbers of individuals scoring low, moderate, and high on SDO) could be emailed and invited to sign up for the study. On average, those who agreed to be contacted about the future study were lower in SDO $(M=2.62, S D=.94)$ than those who declined $(M=2.98, S D=.96$; $\mathrm{t}(499)=3.95, p<.001$, Cohen's $\mathrm{d}=.38)$.

I determined SDO group membership using a quartile split. Altemeyer (2003, 2004) similarly used this method, classing individuals scoring in the upper quartile as 'high-SDOs'. Overbeck, Jost, Mosso and Flizik (2004, Study 1) used this same method, though included only top and bottom quartiles as high and low scorers, respectively (i.e. did not include a moderate/middle group). This research shows that there is a precedent for using quartiles to allocate people into groups based on their responses to the SDO scale. However, the researchers do not report the mean SDO level in each group, so there is no way to compare how similar the groups are.

I verified that the mean level of SDO significantly differed based on group membership using a one-way $\operatorname{ANOVA}(F(3,499)=1945.74, p<.001$, partial $\eta 2=$ .92). Post hoc tests using the Tukey correction confirmed that the mean SDO level in each group was significantly different from every other group (as indicated by all $p$ 's $<.001$, and non-overlapping confidence intervals around the means). Descriptive information about each of the SDO groups is included in Table 5.1 below. 
Table 5.1.

Descriptive information about the SDO groups from preselection data for Study 4.

\begin{tabular}{lccc}
\hline Group & $\mathbf{N}$ & $\mathbf{M}$ (SD) & SD \\
\hline $\mathbf{1}$ (low-SDO) & 135 & 1.53 & .33 \\
$\mathbf{2}$ (low moderate-SDO) & 117 & {$[1.48,1.59]$} & .19 \\
$\mathbf{3}$ (high moderate-SDO) & 129 & 2.36 & .25 \\
& & 3.14 & \\
$\mathbf{4}$ (high-SDO) & 122 & {$[3.10,3.18]$} & .29 \\
& & 43.00 & \\
\hline
\end{tabular}

Phase 2. Based on groups above, with the two 'moderate-SDO' groups collapsed to form one, my supervisor invited equal numbers of individuals who fell into each group to participate in the second study. The interview study consisted of a total of 59 individuals (20 low, 20 moderate, and 19 high in SDO), therefore group sizes were roughly equivalent. In terms of the descriptive characteristics of these participants, there were slightly more males $(N=33)$ than females $(N=26)$, reflecting that a greater number of males were invited (given that the psychology class tends to be around $70 \%$ female). Participants were on average 18.58 years old $(S D=1.69)$. The overall mean and distribution of SDO scores was similar to the larger sample $(M$ $=2.73, S D=1.04)$. Again, the groups differed on mean $\operatorname{SDO}$ level $(F(2,56),=$ $339.06, p<.001$, partial $\eta 2=.93$ ), with each group significantly different from the others (all $p$ 's $<.001$ ), as demonstrated in Table 5.2. 
Table 5.2.

Descriptive information about the SDO groups from those interviewed in Study $4 a$.

\begin{tabular}{lccc}
\hline Group & $\mathbf{N}$ & $\mathbf{M}(\mathbf{S D})$ & SD \\
\hline $\mathbf{1}$ (low SDO) & 20 & 1.56 & .37 \\
$\mathbf{2}$ (moderate SDO) & 20 & {$[1.38,1.73]$} & .22 \\
$\mathbf{4}($ high SDO) & 19 & 2.70 & .26 \\
& & $3.59,2.80]$ & \\
\hline
\end{tabular}

It must be noted that SDO is measured on a 7-point Likert scale (as described in the Measures subsection below). This means that the midpoint of the scale is 4, which indicates that a participant, on average, 'neither agrees nor disagrees' with the items. This is almost the exact mean of my high-SDO group (3.99). This label, while consistent with previous research (e.g. Altemeyer, 2003, 2004) is perhaps misleading, given how the mean is interpreted with reference to the scale.

Overall, average endorsement of SDO in my sample was low $(\mathrm{M}=2.74, \mathrm{SD}=$ .96). To put this value into context, I returned to the meta-analysis data and rescanned through the papers and datasets available to me to gather mean values of SDO to accompany all standard deviations already collected, focusing on only the 7-point scales. An analysis of these results showed no difference between mean endorsement of SDO among student and general population samples $(t(25)=1.12, p=.272)$, however the groups differed in standard deviation $(t(25)=2.36, p=.039)$. General population samples have a greater spread of data $\left(M_{\mathrm{SD}}=1.02, S D_{\mathrm{SD}}=.13\right)$ than student samples $\left(M_{\mathrm{SD}}=.90, S D_{\mathrm{SD}}=.12\right)$.

As demonstrated in Chapter 2, students exhibit a weaker association between their ideological and environmental attitudes (Study 1A \& 1A). However, a relationship between scores on SDO and environmental attitudes is commonly found even among such populations, albeit a weaker association than typically exists among general population samples (Study 1A \& 1B). Therefore, the low scores on SDO do not preclude investigation of how these attitudes relate. Instead, the groups are low- 
and high- in SDO relative to one another, but not in absolute terms with reference to the scale. In this Chapter, I refer to the 'relatively high' SDO group as the 'high-SDO' group. However, I fully acknowledge this limitation and discuss the issue further in the Discussion section at the end of this Chapter.

\section{Measures and Procedure}

The full 16-item $\mathrm{SDO}_{7}$ scale (Ho et al., 2012), as described in the Method sections in Chapter 3, was used in Phase 1 to group participants by mean levels of endorsement. In this sample, the reliability for the full scale was excellent $(\alpha=.90)$.

My supervisor invited individuals who met the criteria for each SDO group so that I could remain blind to the SDO-level of each group. This was to mitigate bias, as my expectations could influence my coding (e.g. experimenter bias or expectancy effects). For instance, knowledge of the aims of this study could have made me more susceptible to coding those I know are higher in SDO more liberally into 'SDOtypical' codes.

For similar reasoning, an independent interviewer blind to the aims of the study was hired to conduct the interviews. The interviewer was experienced in conducting semi-structured interviews, signed a confidentiality agreement approved by the ethics committee, and was under instruction to follow the interview schedule described below. Individuals who accepted the invitation to take part in the study signed up to an interview time via Sona Systems (a subject pool scheduling and management tool).

\section{Interview schedule}

The full interview schedule is included as Appendix D. The interview consisted of six main sections: an introduction, the 'biggest problems' section, then some participants answered follow-up questions to the biggest problems section, and all participants answered the climate change section, which included a series of questions about deep sea drilling, then finished with a conclusion. Although I scheduled 60 minutes for each interview, they typically lasted around thirty minutes $(M=32.59, S D=9.98$, range $=12.32-54.51)$. The length of the interview was not related to participant's SDO level $(r=.00, p=.989)$. 
Section 1: Introduction. The interviews commenced with a brief introduction where the interviewer thanked the participant for taking part in the study and explained the purpose of the interview: to hear their honest opinions about a range of social and political issues. They were also encouraged to read the information sheet, and then sign a written consent form, before commencing an audio-recorded interview.

Section 2: Biggest problems. Next, participants were asked a series of questions about the biggest problems they believe are facing New Zealand and the world, both today and in the future.

Section 3: Follow up to the biggest problems. The interviewer was instructed to follow on from the 'biggest problems' set of prompts by asking followup questions on one of three topics, depending on which flowed best from the responses given in Section 2. This further obscured environmentalism as the focus of the study. If participants mentioned climate change as one of the 'biggest problems', then they would immediately be asked the questions from Section 4 below. Two thirds of participants cited environmental issues in response to at least one of the 'biggest problems' stems. If they mentioned race relations/inequality (for example, mentioning the inequity between NZ Europeans and minority groups), they were asked further follow up questions on this topic. If they mentioned employment, they were asked the same series of questions about this issue.

These two topics were chosen as 'deception' topics as they were commonly given by New Zealand participants in response to the same question used in an unrelated study (Stanley, Millin, Mickleson \& Milfont, 2018). I therefore had reason to believe these topics were likely to come up in this study, especially given that inequality tends to be a top concern for low-SDOs, whereas high-SDOs tend to be more concerned about economic issues such as government spending and unemployment (Duckitt \& Sibley, 2007; Leone \& Chirumbolo, 2008). Finally, if none of the above topics were mentioned, the interviewer asked follow up questions about either inequality or employment, so that the focus on the environment was less obvious.

Section 4: Environmental topic. All participants were asked the series of questions outlined in the interview schedule about the environment, whether they 
were asked directly about this topic (which was the case for participants who cited the environment as an issue on their own volition in Section 2), or if they first responded to questions about inequality or employment in Section 2 . The environmental topic followed the same series of questions as the questions about other issues included above. However, in addition to these questions, participants were asked for their opinions about some additional issues related to climate change.

The interviewer recited an adaptation of the stem from one of the items from Liu and Sibley's (2012) environmental sacrifice scale, "Some people are willing to make sacrifices to their standard of living, for example accepting higher prices, drive less, or conserve energy, in order to protect the environment." This item was chosen as it was also used in the longitudinal research presented in Chapter 3. This initial quantitative finding suggests that high-SDOs may be especially unwilling to make personal sacrifices for the environment, and therefore this could prompt interesting discussion (both from high- and low-SDOs). After reciting this statement, the interviewer asked participants their thoughts on that, and to justify their response (with the prompt, "why?"). She also asked to what extent the participants are willing to make sacrifices for the environment, and finally for their thoughts on the barriers that stop them from doing more for the environment.

Section 5: Deep sea drilling. As part of the discussion on climate change, and following the questions in Section 4, participants were told that the NZ government was looking into the feasibility of deep sea drilling in New Zealand waters. Participants were asked for their thoughts on this, whether they support it, and who they believed should benefit from such an operation if it were to go ahead. Deep sea drilling was chosen as it was a controversial and topical issue in New Zealand at the time, and represents a point of tension between environmental and economic issues.

Section 6: Conclusion. Once the interviewer had worked through the questions in Sections 4 and 5, they ended the interview (regardless of whether the participant answered Section 3 or not). Participants were thanked for their time and informed that they would receive an electronic debrief when all interviews were complete. 


\section{Ethical considerations}

Some deception was involved in the interview study. Specifically, participants were not told why they were selected for the study prior to participation. Furthermore, I took steps to obscure the primary aim of the study (i.e. attitudes towards the environment and climate change) by directing the interviewer to follow up on unrelated topics. It is sometimes necessary to use deception in psychological studies when participant's knowledge of the aims of a study have the potential to influence their performance in the study. In this case, knowledge that they were selected on the basis of their views on equality, or that my main interest is in their views on the environment and climate change, could introduce bias, and encourage socially desirable responding (which I presume at this time means in a pro-environmental direction). Participants discussed, at most, two issues: everyone talked about climate change, and only those who did not mention climate change on their own were asked to elaborate on their own issue first, and then directed to talk about climate change. This ensured that the other discussions were used only as deception and that I was therefore only collecting data that had a purpose.

I opted for a delayed debrief, which was sent after all interviews were complete (up to 2 weeks after the first interview) to prevent prospective participants from finding out about the study aims from those who had already participated. The debrief also included contact information for the researchers (myself and my supervisors) in case participants had any questions.

\section{Analytic strategy}

I transcribed participant's responses to the questions in Section 4 verbatim. I used the Audacity software program (www.audacityteam.org) to control playing sections of the audio recordings, and typed each transcript into a separate Microsoft Word file. Where I was unable to decipher a word or phrase used by the participant, I put my best guess in square brackets. I then imported these transcripts into the software program NVivo for Mac (version 11.4.2, QSR, 2017). NVivo facilitates qualitative analysis of text-based data, allowing the researcher to highlight sections of data and allocate them to codes, which are then grouped by higher-order themes (called 'nodes'). 
The transcription process provided me with a good level of familiarity with the data. However, familiarity is a crucial part of thematic analysis, therefore my next step was to read through each transcript again before starting coding (as recommended by Braun \& Clark, 2006; 2013). I then went through each transcript and allocated sections of data that I judged were relevant or interesting into codes as I came up with them.

In keeping with the aim of this study as exploratory and knowledge generating, I took an inductive approach. I identified ideas that were common within the corpus of data, and used these to inform development of codes (a data-driven approach; Braun \& Clark, 2006). This is in contrast to theory-driven approaches, where the key themes and/or codes are derived from previous literature and preexisting theory before analysis of textual data begins. My approach was also consistent with postpositivism, which is a step removed from the objectivity of positivism, instead acknowledging and attempting to reduce the subjectivity inherent in qualitative research. The use of an independent interviewer and remaining blind to the SDO level of participants during the analysis process are both consistent with this position.

To reiterate one point made above: the average and distribution of SDO scores among Phase 2 participants was similar to the descriptives of the larger group from Phase 1. Therefore, overall the interviewees were not distinctive in terms of ideology, despite being recruited based on their responses on the SDO scale. I approached each transcript without preconceptions, looking across them to interpret codes and higherorder themes within the entire corpus of data (i.e. not separated by SDO group). My approach to each transcript was also iterative: I came up with as many codes as I could identify from the first transcript. I repeated this process for every transcript, sorting segments of data into existing and new codes that I created as I went. I then ran through every transcript again, to ensure that codes created after analyzing earlier transcripts were utilized. I did this a third time, at which point I did not add any additional new codes. In qualitative analysis, this is referred to as reaching the point of saturation (Braun \& Clark, 2013). Once I stopped generating new codes, I was satisfied that all key ideas from the data were captured within the existing body of codes. 
Where extracts were interpreted as belonging to two codes, and these captured similar ideas, I re-considered whether the codes were, in fact, different. In several cases, I decided that the codes were similar enough to be merged. For instance, I had three codes belonging to a theme named It's a Balancing Act (reflecting the idea that environmental actions are often at odds with other interests). Convenience versus Effort, a code defined by the tension participants discussed between 'effortful' proenvironmental action and taking the 'easy option' mapped onto the Motivation code, which similarly included the idea that pro-environmental action takes more effort, so these were combined. Similarly, Weighing Up Costs and Benefits, a code tapping the pros and cons of acting pro-environmentally, almost exclusively captured economic points. Therefore, the code captured the same extracts as the Costs code (for concern about personal costs of pro-environmental action) and the Prioritising Profit code (for acknowledging that pro-environmental action costs businesses).

I also organized the codes into themes and subthemes - higher-order codes used to help organize and represent the patterns of responses in the corpus of data (Braun \& Clarke, 2013). In the section below, I describe the themes that I interpreted from the corpus of data. Throughout this chapter, I capitalize names of themes and codes to differentiate their use from the normal usage of the words involved. To support my discussion, I include up to three representative extracts from the transcripts that illustrate each code. In the analysis section below, I discuss how participant's ideas about climate change fit with the broader environmental psychology literature.

\section{Thematic analysis and discussion}

I concluded my analysis with 33 codes that broadly fit into three main themes. I labeled the first theme Causes and Consequences of Climate Change, as this included discourses about potential antecedents and consequences of climate change. The second theme, Hopeless Environmentalist, captured the personal barriers associated with taking individual action on climate change. The final theme, labeled Solutions, is self-explanatory: it groups together all codes about potential solutions to climate change. This was separated into two subthemes: Top-down (codes suggesting 'action from above' on climate change), and Bottom-up (codes suggesting 'grass roots' action on climate change). 
The hierarchy of themes and codes are included in the thematic map below (Figure 5.1). A thematic map (Braun \& Clarke, 2006) is a visual representation of the structure and relationships between themes (as represented by circles) and codes (rectangles). The two subthemes are linked with thicker lines and housed in rectangles with rounded edges. Dotted lines linking codes across themes depict proposed relationships between codes belonging to different themes. 


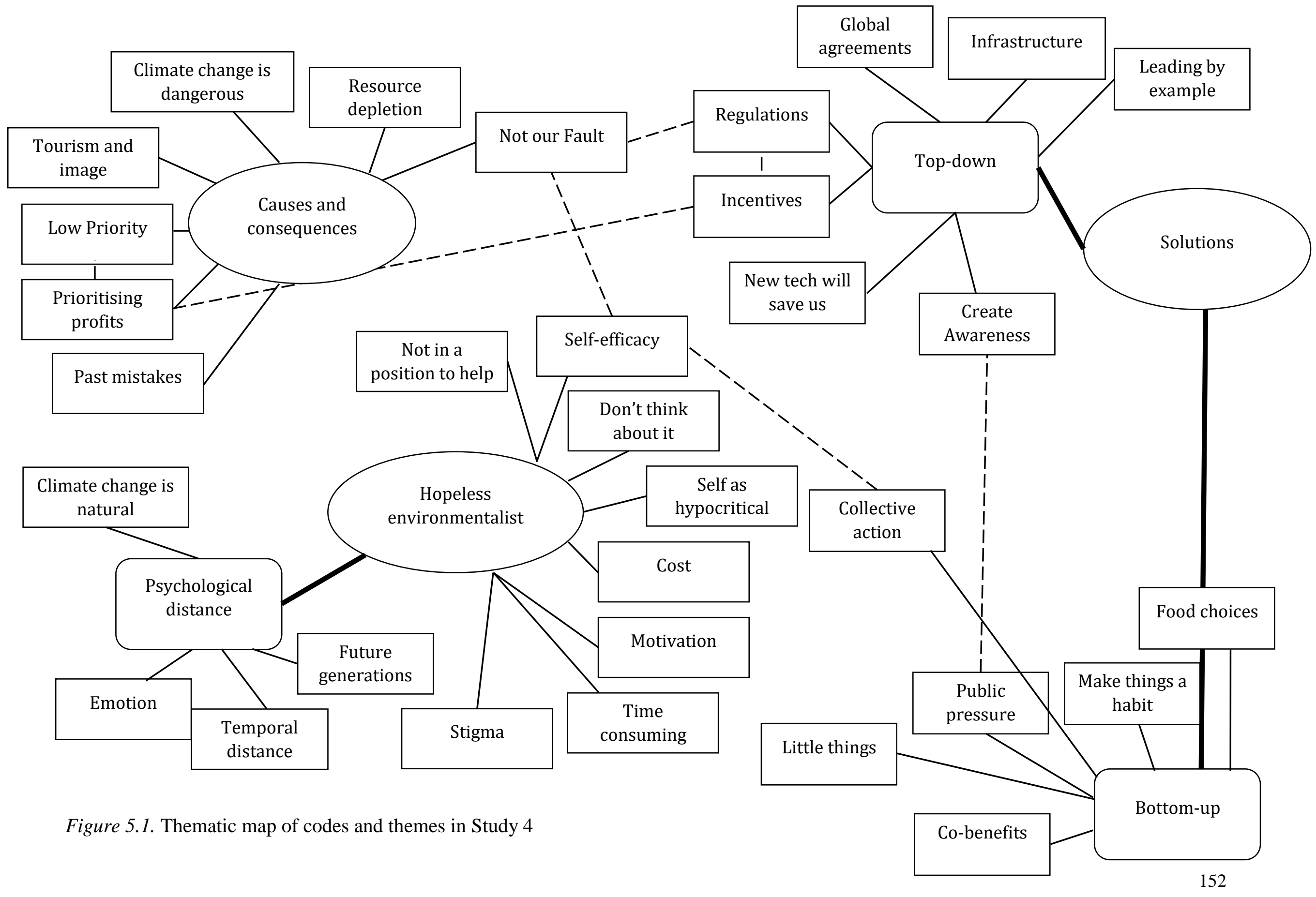




\section{Causes and Consequences of Climate Change}

The codes belonging to my first theme are listed in Table 5.3. This table also includes the number of sources of these codes (i.e. how many participants used this code), and the percentage of participants this corresponds to.

Table 5.3.

Codes included in the Causes and Consequences of Climate Change theme.

\begin{tabular}{lccccc}
\hline & & \multicolumn{5}{c}{ Frequency (\%) } \\
\hline $\begin{array}{l}\text { Causes and consequences } \\
\text { of climate change }\end{array}$ & N sources & Overall & High-SDO & Moderate & Low-SDO \\
Climate change is dangerous & 33 & 55.93 & 47.37 & 55.00 & 65.00 \\
Not our fault & 32 & 54.24 & 57.89 & 55.00 & 50.00 \\
Prioritising profit & 24 & 40.68 & 42.11 & 50.00 & 30.00 \\
Low priority & 23 & 38.98 & 31.58 & 55.00 & 30.00 \\
Resource depletion & 15 & 25.42 & 15.79 & 35.00 & 25.00 \\
Tourism and image & 12 & 20.34 & 21.05 & 15.00 & 25.00 \\
Past mistakes & 11 & 18.64 & 15.79 & 15.00 & 25.00 \\
\hline
\end{tabular}

\section{"Climate change is dangerous"}

Mentions of climate change as dangerous, apocalyptic, and devastating were the most common of the Causes and Consequences theme, which I have grouped into a code called Climate change is dangerous. Extracts from just over half of participants to fall into this category, with many transcripts including more than one extract that portrayed climate change as dangerous.

As an example, Participant 13 backs up their argument about the dangers of climate change by referencing what scientists say about the problem. They use the perceived dangerous and devastating consequences of climate change to justify why people must take action now: 
EXTRACT 1: "It's dangerous, it is wide spread, its effects are starting to be noticed today, and from what I hear, scientists and the people in the know have kind of underestimated how soon the effects can be devastating ... it's gonna change humanity and I reckon that we needa stop, at least stop making more of it now as soon as possible and notice that so I reckon that the dangers of climate change are the effects that it's having on the planet and how little known or cared about it is in today's society." (Participant 13, low SDO).

Participant 16 similarly shows concern for the consequences of climate change, and does so using apocalyptic language (e.g. "doom day") to describe what might happen if we do not address climate change:

EXTRACT 2: "If we don't do anything to kind of slow it down, then there won't be a future for us to live, and basically that's just either we create our own doom day, or nature does it for us." (Participant 16, low SDO).

Participant 55 explicitly related climate change to the movie Mad Max, and speculates on the potential social consequences of the problem:

EXTRACT 3: "I saw a movie the recent Mad Max, have you heard of that? ... I pretty much think that's what the world's gonna turn into ... the planet's gonna be destroyed and then we're gonna be like these savages that are trying to survive and then people are gonna turn real nasty, or even more nasty to survive and compete and dominate." (Participant 55, low SDO).

In each of the extracts where climate change is portrayed as dangerous, deadly, and at times an end-of-world scenario, participant's descriptions might be shaped by media depictions of the issue, as these are frequently characterised by apocalyptic scenarios (for example see Fagan, 2017).

The extracts comprising this code might also be an expression of higher perceptions of risk or climate change anxiety (which was one of the categories of dependent variables in the meta-analyses presented in Chapter 2), with some evidence that these underpin support for environmetnal policies (Smith \& Leiserowitz, 2014), energy conservation behaviours (Lacroix \& Gifford, 2017), and behavioural intentions (Brody, Grover \& Vedlitz, 2012). However, there is also evidence to suggest that the group of people most concerned about climate change are also the 
least likely to engage in pro-environmental behaviour (Hall, Lewis \& Ellsworth, 2018). Therefore, it is unclear if the idea as it is coded in this theme might be related to increased or decreased pro-environmentalism.

Interestingly, the Climate Change is Dangerous code is similar to one code that emerged from another qualitative study probing views on climate change. StollKleeman et al.'s focus groups (2001) showed that compared to groups asked to think of a future defined by strict energy conservation, the business-as-usual scenario returned images of crisis, death, catastrophe, war, and even monsters. While there were no mentions of monsters from my participants, they certainly shared the imagery of devastation and loss of life that Stoll-Kleeman and colleagues' participants associated with climate change.

\section{"Not Our Fault"}

In the next most common code belonging to this theme, Not Our Fault represents discourses about who is to blame for climate change: specifically corporations. Participants whose quotes belong to this code expressed the belief that industries are the main contributors to climate change, and that they must change in order to fix the problem.

Participant 59 provides a good example of this idea, and how both the lion's share of the blame for causing climate change, as well as the responsibility for solving the issue, is placed on businesses:

EXTRACT 4: "Um there's a big thing say with factories and that kind of thing ... there is more of a duty there in terms of if you're part of the problem, you've gotta, you should be looking at how you can contribute towards a solution ... I think if you are going to contribute to the problem then you should be, if only morally, you should be contributing towards an idea of a solution." (Participant 59, moderate SDO).

Arguably, shifting the blame in this way functions to remove personal responsibility for acting on the problem. This is a potentially problematic construction, as it could in turn stifle action on climate change. Indeed, Vail and Motyl (2010) showed that feeling personal personal responsibility for acting on climate change is strongly associated with a greater willingness to make pro- 
environmental changes to one's lifestyle. Therefore, accepting the need to do one's part might be an important precursor to action.

Participants 50 and 57 both stated that while everyone ought to act on climate change, corporations would have more of an impact on the solution as they are the bigger contributors to climate change.

EXTRACT 5: "You see a lot of um like advertising about how you individually can make a difference to the pollution in the world and things and um you know reducing the amount of water that you use or waste or um how much you drive your car to work and stuff, but I think the main contributors are actually the bigger polluters like big factories and um big corporations that aren't doing much to prevent it.... It's y'know individually we can make a difference but it would be very small, um so it's our responsibility to do our own part, but the really big effects is gonna be from big corporations and, yeah.” (Participant 50, moderate SDO).

EXTRACT 6: "Just reducing your impact on the environment, and the bigger you are, the more impact you have, and so those big corporations if they make a change, they'll make changes in other places, and it sort of, sort of a carry on effect." (Participant 57, moderate SDO).

The view that climate change is Not Our Fault shifts responsibility away from individuals. For instance, participants endorsing this code argued that their actions have a minute impact when compared to industry. In holding this belief, they neglect how consumers might, for example, shape demand for eco-friendly products versus those that harm the environment. This position appears to hold both for engaging in environmentally harmful behaviours (as these are 'not so bad', given that corporations do far worse), and for engaging in pro-environmental actions (as the personal sacrifice of doing these actions has seemingly little benefit, given the larger impact of businesses operating as usual). This idea relates to Self-Efficacy, which is discussed as part of the Hopeless Environmentalist theme.

It is also similar to the idea of delegation, where people alleviate the guilt associated with climate change by deflecting the responsibility for environmental degradation onto others, such as political establishments or, in this case, industry (Kollmus \& Agyeman, 2002). Much like the Not Our Fault code, delegation absolves 
people from feelings of personal responsibility, and Kollmus and Agyeman (2002) argue that those who use this strategy are especially unlikely to make any personal sacrifices for the environment.

In a similar theme, Stoll-Kleeman et al. (2001) identified a pattern of absolution they labeled denial of responsibility ("I am not the main cause of this problem", p. 113), similarly linked to arguments about ones own small impact and therefore inability to effect substantial change.

\section{“Low Priority"}

In the Low Priority code, participants cited other issues or commitments as more important (or higher priority) than environmental actions. They may percieve climate change as less pressing, and therefore not requiring urgent action.

For example, Participant 48 criticises the focus on environmental issues, and instead appeals for greater concern about 'human' issues:

EXTRACT 7: "Honestly I get the point of environmental concern, but personally? I feel that we should at this stage, we should only really focus on the environment insofar as allowing human society to flourish in the future, and I don't really, I see like focusing on um breeds of fish possibly dying out, like is a bit misapplied and I personally think, seriously we have way more important things to talk about." (Participant 48, low SDO).

In contrast, rather than labeling these issues as unimportant altogether, Participant 41 explained that climate change is a low priority for people because it is just part of the news cycle:

EXTRACT 8: “I dunno I really don't think anyone's gonna do anything until we experience like a massive global even, like if it just effects one country, I dunno like there's increasing like tsunamis and earthquakes and stuff in countries, and everyone's like 'Oh it's such a tragedy, this place is getting really affected', and like but it doesn't, they read about it in the news and they care about it for a few days, and then it's like oh, and even like shootings and mass murders and stuff like it's a huge tragedy. I mean it lasts forever but it only lasts for some people in our view, cuz then there's the next issue to worry about." (Participant 41, moderate SDO). 
This pattern of attention devoted to climate change, where the issue is low in salience then briefly captures awareness before yet again fading in the public interest, has been labeled the "issue-attention cycle" (Downs, 1972). Egan and Mullin (2017) argue that in the US, climate change is generally an issue of low salience. They use the recent Gallup poll to illustrate this point, where a small minority (1\%) of American's cited environmental problems as the most important problem facing their country (Comparative Agendas Project, 2016). However, while the ebb and flow of climate change news stories and resultant public attention might follow the same pattern in NZ, a quick analysis of the responses to the 'biggest problems' section of the interviews revealed that $25 \%$ of participants in this study cited environmental problems as the most important problems facing NZ today. Therefore, it is not necessarily the case that climate change is low salience in this context. Indeed, in a larger survey of New Zealanders asked the same question, environmental responses were the second-most frequent issue cited (behind 'poverty': Stanley, Millin, Mickleson \& Milfont, 2018).

In a different expression of the Low priority code, in contrast to others who pointed to different issues in the world that are higher priority than climate change, Participant 11 described priorities in their own life that meant they did little towards acting on climate change:

EXTRACT 9: "I'm too busy focusing on study and girlfriend to put too much thought into what I'm doing personally for the problem." (Participant 11, high SDO).

Taken together, what these extracts coded as Low Priority have in common is that other global, topical, or personal issues tend to overtake concerns with climate change in participant's day-to-day lives. Lorenzoni et al. (2007) similarly found among their participants that other issues were reported as more important than climate change.

\section{"Prioritising Profit"}

Building on from the last code, Prioritising Profit includes extracts that frame companies as more concerned about making money than mitigating their environmental impact. A small number of extracts were also allocated into the Not Our Fault code when they also attributed blame to corporations, however the key 
aspect of this code is the focus on profit as the motivator for environmental exploitation. Participants 1 and 13 both implied that businesses are contributing to climate change in a careless way in order to make money:

EXTRACT 10: "I mean there, it seems like there's not like a real effort on the situation, like.. it's kinda just, greed, you know, that they just wanna make profit, they don't really care about the environment, just not caring about like future generations and ..." (Participant 1, high SDO).

EXTRACT 11: "Um I feel like the ones that are causing the problem are just in it for profit, or don't really care about the effect on the world that they're having. Um small businesses I don't really see having an effect either way, um but yeah the massive conglomerates global as well it's just, national they really have to have a sense of morality at some point and realize what they're doing and just change, change their actions." (Participant 13, low SDO).

Consistent with the two extracts above, many participants acknowledged the tension businesses faced between making profits and mitigating impact, and argue that companies will favour making money, irrespective of the environmental consequences. The perception that acting on climate change is necessarily harmful to the economy predicts denial of climate change (e.g. Hoffarth \& Hodson, 2016), so on the surface is a problematic attitude to hold. However, extracts that I included under the Prioritising Profits code tended to oppose the exploitation of the environment for profits.

Participant 9 discussed businesses' preference for maximizing profit, and their reluctance to make changes if it will affect their bottom line:

EXTRACT 12: "Businesses seem very profit driven, especially seeming capitalist society, so I reckon they would be hesitant to make any changes that would reduce product efficiency, so you would have to find a way to show them that being more, I don't know environment friendly, would be just as profitable and efficient if not more than what they're currently doing cuz they would probably not wanna change, it's the whole concept of 'if it's not broken don't fix it', cuz they're making money so why would they wanna change it?" (Participant 9, low SDO). 
This extract suggests that businesses may only act on environmental issues if there are financial incentives. I will return to this idea in the Incentives code from the Top-Down Solutions subtheme.

\section{“Resource Depletion"}

In this code, I grouped together discussions about resource depletion, concerns that resources are being misused, and specific depletion issues such as deforestation and mismanagement of fisheries. Participant 4 expressed concern over resource depletion, putting it down to a mix of carelessness and habit:

EXTRACT 13: “I think it's a symptom of humans, y'know it's like a symptom of us using resources and not replacing them, or using resources without thinking of the effects of what we do, and I think it's caused by a lot of people making heaps of small mistakes and it's just culminating in the current crisis". (Participant 4, low SDO).

Participant 32 acknowledged that many of the 'mistakes' referenced by Participant 4 might have happened before the impact of resource misuse was recognised, however explains that it still goes on:

EXTRACT 14: “Overexploitation as well, just kinda sucking the earth dry for everything we want as opposed to thinking about what's best in the big picture, and I guess a lot of the damage was done before we worked out that there was even a problem, which is another one of the problems." (Participant 32 , low SDO).

One piece of evidence that humans do continue to exploit the environment at increasing rates is that Earth Overshoot Day is only getting closer to the beginning of the year each year. This is the day marking the point each year where humans have taken more resources from the planet than can be regenerated (Global footprint network, 2018). Therefore, despite growing evidence of the problem, humans have only continued to exploit the environment at an increasing rate.

Like many other participants fitting the Resource depletion code, Participant 20 was concerned about deforestation: 
EXTRACT 15: "Probably, y'know driving, gas, um just all that sort of thing and then y'know I don't know too much about climate change, but um y'know big companies that are pumping out fumes and damaging um.. y'know forests and land and all that, just using up all of the natural resources so they can't be used again, um... and I don't know if there's much that can be done to stop that because if we stop that, we're gonna lose something else that we've developed, so yeah it's a tough one." (Participant 20, high SDO).

Participant 20's extract is unique as the only example of its kind that also acknowledges the tension between sustaining natural resources and maintaining built ones, and further highlights the issue of climate change as one characterised by conflicting interests.

\section{"Tourism and Image"}

The next code includes beliefs surrounding climate change as having major consequences for NZ's reputation. This includes comments about the 'clean, green' image we project to other countries being under threat, beautiful parts of the country being ruined by environmental degradation, and the effects these may have on tourism here. Participant 10 explained the effect that environmental consequences of climate change will have on business, and specifically on the tourism sector in New Zealand:

EXTRACT 16: "Our economy actually relies a lot on tourism and if our country is not providing those beautiful environments then we're not really gonna do very well, and like the Earth is fine when we're gone, like it will keep going, but we're not really, not gonna stay around if we can't live on Earth.” (Participant 10, low SDO).

Their extract may be interpreted as indicating less concern about the effects of climate change on Earth itself, and instead concern about the flow-on effects on humans and industry. Echoing concerns that our country's image is at risk, Participant 43 explains that this may deter tourists from visiting:

EXTRACT 17: “I think y'know New Zealand we're sort of displayed to the public, uh the international community is that we're a 'clean green country' and I think that um if there aren't big changes to um how New Zealand's farming industry, and other industries, I'm not sure what the other industry's 
impacts are, but I don't think there's, if there's no changes we're not gonna be 'clean green' for very long and that's something that brings a lot of tourism to New Zealand and improves our public perception, so yeah I think New Zealand faces issues with our physical environment" (Participant 43, high SDO).

The campaign marketing NZ as 'clean, green 100\% pure' was first created (thought to have been around the mid-1980s; Kaefer, 2014) to boost the tourism sector, though it soon extended to our exports, so that these were also marketed as sustainable (Archie, Chapman \& Flood, 2018). However, this image risks becoming tainted by growing concerns about the environmental impact of our animal agriculture industry, the 'clean green' image has been labeled a myth (Coyle \& Fairweather, 2005). Participant 14 referenced the decline of NZ's image related to dairy and its associated emissions, and expressed concern about the impact on our reputation, and consequently business:

EXTRACT 18: “Also there's just the whole issue with y'know New Zealand doesn't seem to be clean and green any more, um y'know dairy contributing heavily to that with all our y'know greenhouse gas emissions and what not, so it seems like our reputation's almost slowly changing, and it seems like we hold a lot of what we are based on our reputation for being y'know obviously quite a clean, healthy environment in society but it seems like that's almost changing and that's going to affect kind of, I dunno I guess how we do especially business, I mean people y'know respect us because of the way that they think we operate but now that it's coming out that we're not as clean as we are, um I see that could be an issue in the future" (Participant 14, high SDO).

It is interesting that for these participants, concern about the impact of climate change on NZ is tied to economic concerns. Indeed, the damage to our environment related to climate change is likely to be serious, likely affecting NZ's exports (including dairy) and services (including tourism) (e.g. Bailey \& Jackson Inderberg, 2016; Fitzharris, 2007). Hsiang et al. (2017) have estimated that continuing with the current global emissions will see poor countries losing up to one fifth of their income within the next 100 years. Meanwhile, in keeping with the unequal distribution of 
consequences of climate change, the United States is set to lose just over $1 \%$ of their GDP per year for every degree the global average temperature rises. These effects are important to note, given that New Zealand will likely be affected by economic impacts on other major economies.

\section{"Past Mistakes"}

The next code of the 'Causes and Consequences' theme groups together extracts that frame climate change as a problem caused by previous generations. In this short and to-the-point extract, Participant 15 responds to the question of who is responsible for climate change with the quote below, which indicates that they believe past generations are to blame:

EXTRACT 19: "Everybody. But most of them are already dead at this point." (Participant 15, high SDO).

Participant 27 similarly blames past mistakes, and contrasts these with the belief that current generations have more sustainable practices:

EXTRACT 20: "I think that like we are speeding it up but it was actually more of like, a previous generation that did it, like y'know the industrial era, like that would have just like killed the environment, um and then like, now everyone's a lot more, y'know environmentally friendly" (Participant 27, high SDO).

Participant 15 similarly blames previous generations, but first excuses this by stating that they might not have known better, before speculating that they may also have acted unsustainably out of disregard for future generations:

EXTRACT 21: "I think it's just the optimistic view of the future from the past, they didn't know any better and I guess they were a bit more careless with what was gonna happen to the world, because they think that it will just last a lot longer, and also maybe they didn't care because by the time anything happened, they would be dead, so yeah." (Participant 15, high SDO).

An emerging consensus is that the industrial revolution and its associated use of fossil fuels and hence greenhouse gas emissions sparked the starting point of a new era of human dominance and exploitation of nature (Fischer-Kowalski, Krausmann \& 
Pallua, 2014). Some trace this back further, to $10,000 \mathrm{BC}$ and the start of the agricultural revolution, which meant changes to land use, expansion of human settlements, and largescale deforestation (Foley et al., 2013; Ruddiman, Ellis, Kaplan $\&$ Fuller, 2015).

However, while the people of the past might have put us on this path, human's negative impact on the natural world continues to be widespread and relentless. For instance, fossil fuel consumption worldwide has increased since the 1950's (Ritchie \& Roser, 2018; Smil, 2017), corresponding to significant increases in global carbon emissions (Boden, Marland \& Andres, 2017). These facts suggest that, while participants might be right in locating the start of the current environmental crisis in the past, it could be problematic to believe that humans are no longer having a serious impact on the environment.

Kurz, Donaghue, Rapley and Walker (2005) also observed interviewees placing blame on previous generations in the context of energy use. In their study, as well as placing blame for pollution on the types of energy produced rather than individual's excessive use of energy (akin to my Not Our Fault code), participants tended to locate blame for pollution with previous generations and their use of coal.

\section{Hopeless Environmentalist}

My second theme, Hopeless Environmentalist, includes codes that reflect the personal barriers to acting on climate change. I include a list of these codes and descriptive information in Table 5.4. 
Table 5.4.

Codes included in the hopeless environmentalist theme.

Frequency $(\%)$

\begin{tabular}{lccccc}
\hline Hopeless environmentalist & N sources & Overall & High-SDO & Moderate & Low-SDO \\
Not in a position to help & 26 & 44.07 & 42.11 & 35.00 & 55.00 \\
Cost & 23 & 38.98 & 52.63 & 25.00 & 40.00 \\
Motivation & 19 & 32.20 & 15.79 & 55.00 & 25.00 \\
Self efficacy & 16 & 27.12 & 15.79 & 25.00 & 40.00 \\
Time consuming & 15 & 25.42 & 21.05 & 25.00 & 30.00 \\
Forgetful or don't think & 14 & 23.73 & 26.32 & 25.00 & 20.00 \\
Psychological distance & & & & \\
$\quad$ Temporal distance & 13 & 22.03 & 26.32 & 35.00 & 5.00 \\
$\quad$ Future generations & 11 & 18.64 & 10.53 & 25.00 & 20.00 \\
$\quad$ Emotion & 11 & 18.64 & 21.05 & 20.00 & 15.00 \\
$\quad$ Climate change is & 8 & 13.66 & 10.53 & 15.00 & 15.00 \\
$\quad$ natural & & & & & \\
Stigma & 11 & 18.64 & 5.26 & 35.00 & 15.00 \\
Self as hypocritical & 10 & 16.95 & 15.79 & 15.00 & 20.00 \\
\hline
\end{tabular}

\section{“Not in a Position to Help"}

The most common code in this theme includes perceptions of being unable to engage in environmentally friendly behaviours because of current circumstances. For instance, participants cited their living situation, financial situation, or time restraints that prevented them from engaging in certain pro-environmental behaviours. This code is perhaps so common in my corpus of data because of the participant pool I drew from: first year students. Many participants mentioned living in a hall of residence, and as such have less freedom to make sustainable choices about their own food, power consumption, or waste disposal (e.g. some residences do not have recycling facilities). For instance, Participant 5 states that living in a hall of residence 
is incompatible with trying to save power, and that they would be willing and able to change their behaviour when their living situation changes:

EXTRACT 22: "If my living situation was different I feel I'd be willing to make more sacrifices but due to, say, living in a hall and such, uh hall of residence with the university, um it's difficult to kinda limit my kind of um my kind of emissions... I'd be willing to make sacrifices in future, once my living arrangements do change, say in a flat um, because yeah I've always like pushed for say like recycling and the whole um composting and such rather than just throwing things away and then like yeah, all that kind of stuff." (Participant 5, low SDO).

While the most common justification for being unable to help was their living situation (specifically for this sample, living in a catered hall of residence), another personal barrier that some participants cited was their financial situation. The extract below illustrates this idea well:

EXTRACT 23: “Well like I don't really have a whole lot of purchasing power, I mean I'm not, y'know a lot of these, well that's not necessarily in terms of the environment but it's y'know society y'know buying from.. um fair trade sort of products I mean I don't have a whole lot of purchasing power so I can't really contribute to making positive changes in that direction." (Participant 14, high SDO).

In addition to being unable to recycle or purchase certain products that might help the environment, a smaller number stated that they were unable to cut out meat as a pro-environmental action, though they would like to as they recognize the benefits for the environment. For instance, Participant 19 expressed the belief that a vegan diet is unsustainable for students:

EXTRACT 24: “Ooh, um I wanna be a vegan, but.. I've searched a lot but I think... that that can't be a very good lifestyle for me right now because I'm a student I use my brain so much and I need to... I need those like animal fat to keep my brain functional." (Participant 19, high SDO).

Not in a Position to Help appears to function as a way of excusing inaction on climate change, as it is seen as out of participant's control. Positioning this 
construction in the broader psychological literature, it is consistent with low perceptions of behavioural control as in the Theory of Planned Behaviour (Ajzen, 1991). Perceived behavioural control is an important determinant of action on climate change, with higher perceptions of control over pro-environmental behaviour associated with behavioural choices that lead to smaller projected increases in global temperatures (Beckage et al., 2018).

While some pro-environmental actions are impossible due to lack of infrastructure (e.g. lack of public transport in some areas, no recycling facilities), some are achievable and instead only perceived as prohibitively difficult. This is reminiscent of Stoll-Kleemann et al.'s (2001) Fabricated Constraints code, where their participants argued that "there are too many impediments" to action on climate change (p. 113) and Comfort code, where behavioural change is perceived as insurmountably difficult. Many of the codes in the Hopeless Environmentalist section are similar to these two codes interpreted by Stoll-Kleemann et al., who grouped together justification for inaction that is seen to be out of one's control, requiring too much effort, time, or money, and therefore a number of barriers to overcome.

\section{“Cost”}

The second most common code within the Hopeless Environmentalist theme is Cost. This code is evidenced in a collection of extracts where participants attribute their lack of action on environmental issues to the high cost of doing so. This is conceptually distinct from the Prioritising Profit code of the first theme, which focuses on company-level decisions made to maximize profits to the detriment of the environment. Instead, this code emphasizes the cost for individuals as a barrier to personal action, and the perception that environmentally friendly options are more expensive.

Participant 11's extract below is representative of this code, as they suggest that environmentally friendly options are more expensive, which is unobtainable for New Zealanders who might not be able to afford to purchase them, despite wanting to be environmentally friendly:

EXTRACT 25: "I mean it probably, it is more expensive to use to pursue options that are more less taxing on the environment, um not many people are in a position to do that and I dunno survive, New Zealand's an expensive 
country to live in um.. so many families are just scraping by as it is so them being required to purchase expensive goods in order to, I dunno ease their conscience towards the environment, is a bit much of an ask, so.. while I think it should happen I don't really think it's going to happen" (Participant 11, high SDO).

When asked what stopped them from taking more action on climate change, Participant 28 responded:

EXTRACT 26: "I try not to think about it because I know I should be doing more but at this time like I don't have heaps of money to buy the like stuff that's, not planet friendly but like better for the environment you know like.. but I feel like if I could I should, so when I have the ability to be able to pay more for stuff that's better for the environment like just little things, I will, but at the moment I just.. I just can't'. (Participant 28, moderate SDO).

While for other participants, the cost of environmentally friendly options might be perceived as too much to afford, Participant 24 instead framed the cost as unjustifiable when taking personal preferences into account:

EXTRACT 27: "Um I think yes sacrifices are definitely good to be made at an individual level, but I don't think it should strip away your needs? So like if I was to decide between spending six dollars to get into town for uni compared to maybe twenty minute drive and like having a good parking spot because then I have other things to do during the day and I have to get around town, I don't wanna spend another like three dollars getting around town that's like, I wouldn't waste that much time and money? Like I feel like time is more important, money I can afford to maybe sacrifice a little, but it's still up to what I need, it's not about the environment." (Participant 24, high SDO).

Taken together, the extracts supporting this code suggest that some proenvironmental action is perceived as costly for individuals. This code is perhaps again common among my participants given that students are a group who tend to have scarce financial resources, and thus pro-environmental actions that do cost money require a trade-off between personal financial wellbeing and prosocial behaviour that might not have immediate personal benefits. 
Cost has been shown to represent a significant barrier to acting on climate change. For instance, schemes that require users to pay to recycle result in lower levels of recycling (Seacat \& Boileau, 2018). Furthermore, initiatives that remove costs see an increase in pro-environmental actions, such as free bus passes increasing the use of public transport (Laverty, Webb, Vamos \& Millett, 2018). Some proenvironmental actions are more accessible to those with low incomes and even include financial benefits, such as energy conservation (Chen, Xu \& Day, 2017), which will be discussed in the Co-benefits code.

\section{“Motivation”}

Motivation captures the framing of environmentally friendly actions as requiring more effort than their alternatives. Some participants explained that they avoid the environmentally friendly option because they "can't be bothered", say they are too lazy, or lack the energy or motivation to engage. All of these reasons were grouped into the Motivation code, as all demonstrate that lack of motivation is a barrier to action on climate change.

In this first example, Participant 36 acknowledges laziness as one factor that makes people resistant to changing their use of cars for personal transport:

EXTRACT 28: "Yeah I mean like just what I was saying then like driving a car or um getting around, cuz so many things are being developed y'know all this technology to make life easier, but technically it's just cuz we're lazy [laughs] essentially but it's become so like engrained in our lifestyle that it would be so difficult for us to revert back to y'know before all this, yeah." (Participant 36, moderate SDO).

In the next set of extracts, Participants 57 and 4 do not focus on any specific behaviour. Instead, they speak generally about lacking the motivation to make changes for the environment:

EXTRACT 29: "I think people have just gotten into the habit of, if something's how it is, we just keep it as it is, we don't want, we want change but we don't want to make the change, so we want things to be better but we just, we can't be bothered to make it better." (Participant 57, moderate SDO). 
EXTRACT 30: "I know that I'm probably doing things, lots of things really lazily and being like 'Ah, nah someone else will do it', y'know it's easy to get into that mindset, especially if you're stressed and tired." (Participant 4, low SDO).

Laziness has been identified as one individual barrier to turning environmental values into action (Singhirunnusorn, Donlakorn \& Kaewhanin, 2017), so again this reported personal barrier to action fits with those reported in the literature.

\section{"Self efficacy"}

The next code represents ideas about action on climate change being unlikely to make a difference. This is a common idea in the environmental literature, with the perception that one's actions are likely to make a difference significantly associated with engagement in pro-environmental behaviour (e.g. Bamberg \& Möser, 2007), and the reverse true for individuals possessing low self-efficacy beliefs. In the extracts below, the participants express pessimistic views of the effectiveness of individual action on climate change:

EXTRACT 31: “Um, uh because my actions won't count for naught when compared to others, so there needs to be a sort of collective motivation to do something”. (Participant 18, moderate SDO).

EXTRACT 32: “Um.. One person doing it, I dunno it's like a grain of sand on a beach isn't it like one person isn't really like gonna do much, but everyone is gonna do a lot, both positively and negatively, like if everyone starts saving these things then it's gonna make a massive difference but if everyone just doesn't then it's definitely gonna have a drastic impact." (Participant 41, moderate SDO).

In these cases, the participants state that individuals' actions alone will not make a difference. However, they may still maintain positive attitudes towards proenvironmental behaviours, as they speculate that action on a larger scale will make a difference (therefore perhaps instead holding beliefs about collective efficacy, e.g. Reese \& Junge, 2017). Participant 20 articulated a similar view:

EXTRACT 33: "My mum recycles and it's like, that's great but you're one person, and I know that whole saying oh y'know, one person will do it then 
the next person will do it, but that's never gonna affect everyone in the world, so I really think that .... Something needs to be done from a really high up level if anything is to be done about... y'know slowing it down”. (Participant 20, high SDO).

While Participant 20 shared the view that these behaviours need to be more widespread in order to make a difference, their extract also implies that widespread or global action is impossible (i.e. low perceptions of collective efficacy). Instead, they advocate for top-down rather than bottom-up solutions on climate change.

In general, participants who suggested that individual actions on climate change were unlikely to make a difference justified this stance by pointing out that action needs to be more widespread in order to make a difference. Some participants appeared to use this to excuse their nonaction, given that action would not make a difference anyway. This is consistent with feelings of powerlessness: believing that changing one's own behaviour has no effect on whether climate change happens or how serious it is, which in turn predicts lower engagement in action on climate change (Aitken, Chapman \& McClure, 2011). Indeed, this was the label that StollKleemann et al. (2001) gave to a similar code interpreted from their own corpus.

The perception of powerlessness and low self-efficacy in this scenario likely occurs because the decision of whether to act on climate change or not represents a social dilemma. Social dilemmas represent situations where acting in one's own best interests has clear benefits but is overall detrimental to the community as a whole, which benefits the most if everyone cooperates (Dawes \& Messick, 2000). At the individual level, environmental inaction has clear benefits (e.g. greater share of the resources), while cooperation in the form of engaging in pro-environmental behaviours represents a significant personal sacrifice (e.g. reducing consumption of resources and energy). The danger here is that when inaction on climate change is scaled up to the extent that everyone is doing it, the environment and community as a whole is at serious risk in a tragedy-of-the-commons scenario. For some people interpreted as using the Self-Efficacy code, the benefits of cooperation to society are unclear, which might sway them instead towards inaction. 


\section{“Time Consuming”}

I coded extracts into the Time Consuming code if the participant labeled environmental actions as taking more time, or stating that they do not have the time to take them (which in turn implies that these actions are more time consuming). For Participant 7, both time and money are personal barriers:

EXTRACT 34: "Um... I think time.. and money is probably a bigger one, but like for most things you don't actually need money, you just need time but then there's those ones where you do like sort of need money, which is probably the things that put me off." (Participant 7, moderate SDO).

In the exchange below, Participant 16 (low SDO) discusses the amount of extra time it takes to bus to University as the main barrier to using public transport:

\section{EXTRACT 35:}

Participant 16: "I would definitely use public transport all the time if the public transport was better, yeah if they fixed that as well."

Interviewer: "So what part of the public transport.. like not, y'know what part of it's difficult for you?"

Participant 16: "I think it's just the.. it's not very regular, and it's not um.. very fast either, it's very slow and time consuming, um if they create some sort of rail that would be quite cool."

Interviewer: "So is that for you coming from like home to uni?"

Participant 16: "Yeah, I mean there is a bus from like, the [name of bus] which goes from [location] up through here and South but um, it's not like certain times it's quite regular but other times it's not regular and it's very slow and it's always full and it's just very exhausting, yeah."

In this extract from Participant 17, there is similarly the implication that having a positive impact on the environment is time consuming:

EXTRACT 36: "Yeah well I was just about to say like personally I'm not, like I feel like I am pretty reliant on everything that I use, and at the moment I feel like I'm already like more preoccupied by coming to uni and getting my 
studies done that I wouldn't, I'm not as willing to y'know spend extra or take more time out of my life to make a good impact, even though I feel bad about that, y'know." (Participant 17, high SDO).

While some participants said they did not mind spending more time if they knew it would make a difference, Participant 17 stated that taking the extra time for the environment is too much of a sacrifice for them.

\section{“Forgetful or Don't Think"}

This code includes extracts discussing how the participants do not consider the environmental impact when they are making decisions, or admissions that climate change and the environment are not 'top of mind'. In the extract below, Participant 12 suggests that climate change is not something we think about every day:

EXTRACT 37: "Um I think just the routine mundane like, this is just what happens, and people don't really give much thought to it until they're like provoked to think about it." (Participant 12, moderate SDO).

Participant 47 appears to share this belief, suggesting that instead their actions are determined by what they enjoy without thinking about the environment. However, they state that do consider changing their behaviour when climate change is top of mind:

EXTRACT 38: "It's just.. the kind of thing that I like to spend my time with, it's.. I don't really think about it much, like in my day to day life, but then the times that I do think about it I'm like oh I should really stop, and I just don't, um.." (Participant 47, high SDO).

In contrast to this, climate change similarly does not enter into Participant 2's decision-making process, and they take this a step further to suggest they do not care:

EXTRACT 39: “Oh, I think it's the [pressingness?] of it, it's not in my face in a way that I can really be scared about. Um, I kind of have a view that the worlds, kind of, going to hell in a hand basket anyway so, do I really care? [laughs], maybe, I dunno it's kind of, it's just, it's not necessarily a conscious thought it's kinda just lingering in the background." (Participant 2, low SDO). 
Crucially, this code suggests that while individuals might accept that climate change is happening, it may not necessarily enter their decision-making process when they are making choices that affect the environment. Therefore, for those who do not have the environment in mind when making decisions, the low salience of climate change means pro-environmental attitudes are unlikely to shape pro-environmental behaviour.

Environmental awareness is part of the intention-action gap (Kollmus \& Agyeman, 2002), however this concept is defined as understanding one's impact on the environment. Rather than a lack of knowledge or understanding, participants endorsing the Forgetful or Don't Think code are not conscious of their impact on the environment while making decisions, resulting in some environmentally harmful behaviours. This different kind of lack of awareness could potentially help to explain another portion of the disconnect between pro-environmental intention and climate change inaction.

\section{“Psychological Distance”}

Climate change is said to be pyschologically distant across four dimensions (Spence et al., 2012): geographical distance (effects will occur in locations further away), temporal distance (further into the future), social distance (affecting people unlike ourselves), and uncertainty distance (effects are not certain). From the interviews, I found evidence of perceptions that climate change impacts are far away in time, which I separated into two codes: time and future generations. The Temporal Distance code captures the framing of effects as distant, or occuring in the future, which is consistent with Spence et al.'s (2012) original definition. I also identified a new aspect that seems conceptually related to temporal distance. The Future Generations code is given to extracts that report concern for how climate change will affect future generations, or mentions of needing to act on climate change for the benefit of future generations.

The Climate Change is Natural code is similar to the uncertainty dimension of climate change. However, in my study the specific aspect of climate change that participants call into question is the anthropogenic nature. This could tap into a different aspect of psychological distance altogether: distancing oneself from the causes of climate change. 
The other psychological distance code that I interpreted from the data is not part of Spence et al.'s (2012) original conceptualisation of psychological distance, while being consistent with it: Emotional Distance. This code was used for extracts where participants talked about apathy towards climate change or environmental issues being a problem: they think it is a problem that others do not care. Very few participants said that they themselves did not care (but see extract 42 above), and therefore the code was applied to extracts where participants expressed concern over others being emotionally distant from the problem.

\section{Temporal Distance.}

Participant 51 did not appear to express concern about any immediate effects of climate change, as they believed that the effects are temporally distant:

EXTRACT 40: "Um oh because it's gonna maybe not very soon but in a long amount of time it's gonna affect the way that we live, and it's gonna.. it's not gonna be good, it's going to yeah affect our way of life, it's gonna.. destroy areas and live and animals and um.. yeah it's just not gonna make the world last." (Participant 51, high SDO).

Participant 42 made reference to the variation between when we might experience effects of climate change (far into the future), and when low-lying island nations may see the effects (temporally close):

EXTRACT 41: “Um I'm.. um.. climate change possibly a lack of um urgency I guess around the matter, um well world wide urgency there's certainly things from say the Kiribas they might effectively just.. they might be flooded over, they're already saying that they've, they're experiencing effects of um.. well the ocean level beginning to rise I guess and um most areas there are sort of only one or two meters above sea level so they're going to be majorly affected, whereas larger countries they'll, they're not really seeing the problem.” (Participant 42, moderate SDO).

In the next exchange, Participant 14 (high SDO) also sees climate change as a problem for the future (temporally distant). 


\section{EXTRACT 42:}

Participant 14: "It takes a bit of time but once the ball starts rolling, and the environmental effects, cuz they are delayed for about I think a week we learnt in FCom but I mean yeah I dunno say a hundred years the effect, the damage we do now then will be apparent but I'd say one of the biggest barriers yeah also is just ignorance I mean people y'know.. don't really know I guess." Interviewer "And so do you think it's a problem?" Participant 14: "Well I mean it's not, no not short term, long term yes, short term I mean it seems not so bad."

Spence et al. (2012) concluded that greater perceived temporal distance was associated with lower concern about climate change and willingness to conserve energy. Therefore, perceiving climate change as distant in time or in any other dimension might indicate that a participant is unlikely to engage with the problem, and lowering such perceptions might be a good avenue for future research (e.g. as in Jones, Hines \& Marks, 2017).

\section{Future generations.}

In response to the interviewer asking who is responsible for finding solutions to climate change, Participant 56 justified their belief that everyone should contribute to the solution by pointing to the effect climate change will have on future generations:

EXTRACT 43: “Um I say it's, or if we wanna provide a liveable earth for future generations I think it's everyone's, everyone should be concerned with it." (Participant 56, moderate SDO).

In the next two extracts, the participants explain how our inaction on climate change today has repercussions for future generations:

EXTRACT 44: "Well like obviously what we are doing is ruining like our world and everything and we're kinda just pushing it off like ignoring it I guess like we need to start realizing that it is a happening thing, cuz people are like 'Climate change doesn't exist' but it like obviously does like it's scientifically proven to, and like what we are doing isn't, we aren't making 
major changes to prevent this from happening, it's just kind of pushing it off to the next generations ... and yeah I guess we're ruining the world for the future people." (Participant 38, moderate SDO)

EXTRACT 45: "Well people are just more concerned about themselves, like we're very selfish, like we are, and people are just more concerned about like what's best for them, or what's best for me, or y'know, and I feel like there's this whole like 'Oh, we've gotta build for the next generation', but it's like, you're building for a generation that's gonna not have enough trees to what, breathe? Like, or y'know not gonna have nice little animals where you can go like and see them or you can go in cool walks in the park or something like that." (Participant 27, high SDO).

None of the distance dimensions examined by Spence et al. (2012) made reference to future generations. However, extracts belonging to this code did express a perception of temporal distance (as the effects will be felt by generations to come). There is little research to date on how concern for future generations fits within the broader environmental psychology research. However, some researchers have examined whether parenthood (and therefore, an implied investment in future generations) is associated with environmentalism, with mixed results. For example, parenthood is not related to an increase in pro-environmental attitudes or behaviour (Thomas, Fisher, Whitmarsh, Milfont \& Poortinga, 2017), however it is slightly associated with increased worry about climate change (Ekholm \& Olofsson, 2016).

\section{Emotional distance.}

Participants were interpreted as referencing the emotional distance of climate change when they discussed being concerned that some people (i.e. not themselves) do not care about the problem. In the exchange below, Participant 36 (moderate SDO) argues that emotional distance is widespread:

\section{EXTRACT 46:}

Participant 36: "Well.. obviously there's a lot of things be can cut back on, um but it's almost impossible without having everyone on the same page, and there's a lot of people who just don't give a shit."

Interviewer: "Yeah why do you think that is?" 
Participant 36: 'I think they're just... ignorant and they don't care that after they die the world's screwed, they're in this little bubble and like a lot of people are like that, it's all you know 'This is my life and I need money and I need this and this is how I'm gonna get it, screw the rest', I guess."

Participant 44 discusses how emotional distance leads to inaction on the problem, and frames this as personal choice:

EXTRACT 47: "Well I guess the thing is it does come down to like your personal opinion and where you individually stand on climate change, I mean there's always gonna be people who say 'Hey, I don't care, I'm just gonna drive anywhere in my like two liter ridiculously overpriced gas guzzling machine like y'know', then there's like the people that are gonna say like 'Hey, we can walk there, we can do this, we can do that', and I guess... it comes down to your own view because as much as like people are gonna be like 'Oh it's unfair that I'm making this sacrifice', but that's their choice, that's their choice to try and contribute, and if someone else doesn't want to that's their prerogative." (Participant 44, moderate SDO).

As well as explaining that others do not care about climate change, Participant 3 suggests that people would care (and emotional distance would reduce) if and when it starts to affect them:

EXTRACT 48: "If suddenly we have floods happening, I dunno, I dunno we have no food and ah because of the pollution, all the stuff we're doing, all the crops that they're.. And then we're gonna say 'Oh we need to find a solution', we don't care until we're pushed. It's like, we're not gonna find another alternative to, y'know fossil fuels, until we're pushed, we have no other choice. And I think that's one of our characteristics of being human.” (Participant 3, high SDO).

This code is reminiscent of the idea of apathy over climate change. Hall et al. (2018) imply that climate change apathy is linked to perceived temporal distance, as they argue it occurs because people "do not percieve climate change as an urgent problem" (p. 55). 


\section{“Climate Change is Natural"}

A relatively less common code belonging to this theme was one that captured beliefs about climate change as a natural process. This code was organised into the 'Psychological distance' subtheme because it is similar to uncertainty dimension of psychological distance (as in Spence et al., 2012). Rather than outright denying the existence of climate change in the interviews, participants whose extracts contributed to this code instead tended to deny or question the anthropogenic nature of climate change. They tend to do so by emphasizing that current and projected changes in the climate are due to natural or 'normal' fluctuations.

For instance, Participant 18 expresses this view in the extract below. Interestingly, they make it clear that this is their own opinion, though explain the rationale for this belief as though it is a scientific explanation:

EXTRACT 49: "Environment I don't believe is much of a problem anymore, it's interesting because um, I'm of the mind that um people um, that the world it goes through a cycle of an ice age and everything every ten thousand or so years, and I just believe it goes through the cycle, the globe gets hotter, the ice caps melt, the sea gets colder, it freezes over, all over again over the course of ten thousand years, and we're not really doing anything, just accelerating the rate at which the next ice age would come, so it's not really an issue for us". (Participant 18, moderate SDO).

Informed by their own 'scientific' sources, Participant 27 also expresses this view in the extract below:

EXTRACT 50: “Um [laughing], I don't actually believe in climate change, like well I don't not believe in it like I can see what's going on like we've had a very warm winter, but like I just like, there's so many like scientific things that I have read that disprove it, and if you look back across the world like thousands and thousands of years ago the Sahara desert was a rainforest, and like the world's moving all the time, like it's constantly changing, it doesn't necessarily mean that it's y'know to do with like the carbon dioxide or whatever, like I feel like it's just a thing the world does, like there's been an ice age at like the same point ish every so many years." (Participant 27, high SDO). 
From this extract, Participant 27 distrusts the vast majority of climate scientists who argue there is sufficient evidence that climate change is happening and caused by humans (Cook et al., 2016; Doran \& Zimmerman, 2009), and instead favours their own scientific sources that 'disprove' this. It is possible that this stems from a distrust in mainstream scientists, which Hornsey and colleagues (2016) identified as one of many determinants of climate change belief, and van Valkengoed and Steg (2019) linked to climate change inaction. This extract, therefore, supports quantitative findings of a statistical relationship between endorsement of climate change and trust in scientists.

Instead of outright denial of the anthropogenic cause, Participant 22 remains agnostic as to the causes of climate change. They explain the two sides they have heard, and claim they do not have sufficient information to know which to believe:

EXTRACT 51: “Okay.. so.. I don't have like a definite opinion, I think, so the two main, so I don't think that nothing's happening, I think y'know there's enough scientific evidence to prove that's not true, but then there are two views and one of them is that like 'Humans are doing this, only humans are doing this, we suck', and then one of, the other view is that, y'know the world goes through a cycle, we're either speeding the cycle up or this is the normal cycle.. shit out of luck it's gonna get hot, like y'know like sorry, poo out of luck, anyway so I think like those are the two main views and I don't know which one's true, so I think whether or not it's the Earth's natural process and we're increasing it or whether or not we're making it ourselves it's still, let's just decrease what we're doing even to like, take it back to its natural course or to stop it, like I'm not sure what doing nothing will do to it, so I do believe in it I just don't believe.. I just don't know which argument of it to believe." (Participant 22, high SDO).

This view could be exacerbated by media representations of anthropogenic climate change as a debate (but see the explanation in the discussion of the Awareness and Education code below). Thus, although the majority of climate scientists are in agreement with the first explanation Participant 22 provides, an even-handed appreciation for each 'side' of the issue may mean that this participant has given equal weight to the second explanation. It is also possible that participants who 
oppose or are uncertain about the anthropogenic nature of climate change are seeking out or attending to different sources of information, including those that confirm their belief (e.g. confirmation bias; Taber \& Lodge, 2006).

\section{"Self as Hypocritical"}

The Self as Hypocritical code is used where participants acknowledge that action on environmental issues is important, but also admit that they do not do much, are guilty, or hypocritical, and hence fall under the Hopeless Environmnentalist umbrella theme. Participant 15 outright labels their behaviour hypocritical:

EXTRACT 52: "See my thoughts on that are moderately hypocritical [laughs] because while I am fully in favour of, y'know making sacrifices towards climate change, towards fixing it, I'm gonna be completely honest and say I probably wouldn't if they inconvenienced me a great deal." (Participant 15, high SDO).

Participant 11 also acknowledges the hypocrisy in their interview, where they were supportive of pro-environmental action, while not following through in their actions:

EXTRACT 53: “Um.. I've talked a lot about um people's effect on the environment um and it may seem like I'm some sort of big advocate, but personally I don't think my actions and day to day impacts on the environment would differ much from anybody’s, um so..” (Participant 11, high SDO).

Participant 52 identified climate change as the biggest important problem facing the world (in Section 2 of the interview). Despite this, in the final extract to back up the Self as Hypocritical code, they admit their own inaction on the problem:

EXTRACT 54: "Um... well this is not, yeah see it's not really on purpose but I don't think I've been in a car at all this year, yeah, or bus yeah just been walking everywhere, and.. fuck.. there's not really much I'd say off the top of my head that I really do, I mean it's quite hypocritical I'm saying this is the most important thing in the world but there's not really much that I do in order to help.” (Participant 52, high SDO). 
This theme appears to be an acknowledgement of the attitude-behaviour gap (also called the value-action gap), which is the difference between acknowledgement of climate change and acting on the issue (e.g. Kollmuss \& Agyeman, 2002). There are numerous reasons for this inaction, and many of them are discussed in this Chapter, such as personal (e.g. cost) and institutional barriers (e.g. lack of public transport, recycling facilities).

\section{"Stigma"}

Somewhat less common is the Stigma code, where some participants articulated the view that being seen to act pro-environmentally attracts judgment, criticism, or otherwise makes it harder to fit in with others. Some specific proenvironmental actions that participants cited as being accompanied by stigma include cycling and going vegan, for instance, as vegans tend to be stereotyped as preachy and moralizing (Minson \& Monin, 2012). In the extract below, Participant 25 explained that stigma stops them from taking action on climate change:

EXTRACT 55: "Um I know for me it would probably be laziness, maybe not wanting to be seen as really outspoken or really um, like hippy I don't know [laughs]." (Participant 25, high SDO).

When asked what individuals should be doing about climate change, Participant 42 made a suggestion about cycling, then noted the stigma applied to cyclists in NZ:

EXTRACT 56: “Um.. obviously it's not going to be the best thing for every single country, but I guess if we take the Dutch approach and just ride bicycles everywhere that might be best, um.. for instance just imagine say what a city like New York would um what their emissions would cut by if every even third person took a bike to work instead of a bus or a car, um.. maybe we don't have.. maybe the culture isn't sort of as friendly towards cyclists and perhaps they just don't have the facilities for that, um but yeah that would be a start I think." (Participant 42, moderate SDO).

As well as cyclists being the target of hostility and frustration for car drivers (e.g. Rissel, Campbell, Ashley \& Jackson, 2002), other barriers that have been identified to cycling include the belief that cycling is a non-normative behaviour and 
that it is for children and old people (Allison, Woodall, Armannsdottir \& Pich, 2017). In a more subtle quote from this code, Participant 2 expressed admiration for those who made sacrifices for the environment, yet implied that they may face stigma given that doing so is a barrier to 'fitting in':

EXTRACT 57: "Um I think those people are um, are great and are kinds of I guess, heroes in a way, um, because it is much easier to live um, a suburban life that um, means you fit in more." (Participant 2, low SDO).

Concerns about the stigma associated with environmental action may reflect that pro-environmental behaviour is non-normative. For instance, if you think you will stand out by doing it, this implies you believe the behaviour is not common. Concern about being labelled a 'hippy', vegan or cyclist suggests one thinks that society does not approve of these groups and their behaviours. Social norms, our beliefs about how common or socially desirable behaviours are, have powerful effects on behaviour (e.g. Abrahamse \& Steg, 2013; Cialdini, 2005).

In their corpus of data, Lorenzoni et al. (2007) also found references to social norms surrounding how people ought to live (e.g. having a car) and undesirable lifestyles (eco-friendly living as 'hippy'). Therefore, although the 'Stigma' code was relatively uncommon in my corpus of data, it does have a basis in the broader literature.

\section{Solutions}

In their interviews, participants put forward many ideas for tackling the problem of climate change. One major distinction in the kinds of ideas put forward was whether the actions they suggest would come from the top (with suggestions of 'action from above') or from the bottom (e.g. grassroots action). I therefore separated this theme into these two subthemes: Top-Down solutions, and Bottom-Up solutions. The codes allocated to each subtheme are included in Table 5.5. 
Table 5.5.

Codes included in the solutions theme.

Frequency (\%)

\begin{tabular}{|c|c|c|c|c|c|}
\hline Solutions & $\mathrm{N}$ sources & Overall & High-SDO & Moderate & Low-SDO \\
\hline \multicolumn{6}{|l|}{ Top-Down codes } \\
\hline Regulations & 36 & 61.02 & 52.63 & 65.00 & 65.00 \\
\hline $\begin{array}{l}\text { Awareness and } \\
\text { education }\end{array}$ & 35 & 59.32 & 57.89 & 75.00 & 45.00 \\
\hline Infrastructure & 29 & 49.15 & 47.37 & 55.00 & 45.00 \\
\hline $\begin{array}{l}\text { New technology will } \\
\text { save us }\end{array}$ & 21 & 35.59 & 47.37 & 25.00 & 35.00 \\
\hline Leading by example & 15 & 25.42 & 15.79 & 30.00 & 30.00 \\
\hline Global agreements & 12 & 20.34 & 10.53 & 20.00 & 30.00 \\
\hline Incentives & 9 & 15.25 & 21.05 & 10.00 & 15.00 \\
\hline \multicolumn{6}{|l|}{ Bottom-Up codes } \\
\hline Little things & 31 & 52.54 & 42.11 & 55.00 & 60.00 \\
\hline Collective action & 31 & 52.54 & 57.89 & 55.00 & 45.00 \\
\hline Co-benefits & 26 & 44.07 & 52.63 & 35.00 & 45.00 \\
\hline Food choices & 21 & 35.59 & 36.84 & 20.00 & 50.00 \\
\hline Public pressure & 16 & 27.12 & 36.84 & 15.00 & 30.00 \\
\hline Make things a habit & 10 & 16.95 & 15.79 & 15.00 & 20.00 \\
\hline
\end{tabular}

\section{Top-Down codes}

\section{"Regulations"}

The Regulations code includes extracts arguing for policy and/or legislation mandating pro-environmental action. The majority of participants had at least one extract coded as representing support for Regulations, making this code the most common in the Solutions theme. Participant 13 took a strong stance on the role of 
government in mitigating climate change, with the suggestion that they should legislate against pollution:

EXTRACT 58: "I feel like having a responsible government which its primary goal is to reverse climate change or at least lessen the effects that New Zealand has on it so that would mean more research money going into reversing the effects of climate change and passing laws and regulations against you know pollution and everything in New Zealand, For the world in general I think yeah, restrictions on pollutions all the way around." (Participant 13, low SDO).

Participant 22 also stressed the importance of regulation, though instead of regulating business, they discuss how laws could be put in place to enforce personal pro-environmental action:

EXTRACT 59: "I mean that's why I think legislation is really important because if there's a law saying something you're more likely to follow it then like based on yourself, so I think it's like laws need to be created that have a small sacrifice on everyone now, and people might not necessarily love the idea of, but then they're kinda forced into being environmentally which in the end they'll like more." (Participant 22, high SDO).

Participant 38 shares this view, extending the notion to cover even more behaviours:

EXTRACT 60: "Um I think there should be like more laws that do prevent people from doing these kinds of things.. I guess it is hard because we're still living in this idea that it's not something that's going to affect us, and it's like you need to realize that to do it now is to help those in the future, so yeah I guess any kind of.. like law that prevents people from doing even the smallest amount of thing that is affecting our lives or our world is like going to make a difference in the long run." (Participant 38, moderate SDO).

Lorenzoni et al. (2007) found evidence of a similar theme, which they called 'Externalising responsibility and blame' Their code was much broader, however, relating to beliefs that business and government are to blame and most responsible to act on the problem, therefore also relating to my Not Our Fault code. However, the 
illustrative extracts presented in the paper only support the interpretation that industry and the US is to blame, so the focus in my study on action from the NZ government appears to be unique.

\section{“Awareness and Education"}

The second most common top-down solution was Awareness and Education. This included suggestions for climate change education or awareness campaigns, teaching about climate change in school, or highlighting that lack of knowledge is the problem. This is a commonsense response - if people are aware of the issue (and potential solutions) then why do they not act upon that knowledge? Thus, a knowledge deficit is responsible for the lack of mitigating action. However, Hornsey et al. (2016) showed that education in general has a negligible association with belief in climate change, and that self-reported knowledge on climate change has a small relationship, in turn slightly overshadowed by objective measures of knowledge.

Despite this, attempting to increase knowledge (e.g. through educational campaigns) is ineffective (Delicado, 2012; Marteau et al., 1998). Therefore, the suggestion that awareness and education is the key to solving climate change is in direct opposition to growing lines of research that show that this is not the case, yet supports a 'commonsense' understanding of the issue. Participant 4 discusses the work that is already underway educating young people about climate change, and suggests expanding this to reach all ages:

EXTRACT 61: "Um.. Well.. I mean I remember in my year nine social studies experience, I actually, we did a climate change topic, and so obviously the New Zealand government has fully accepted climate change, it doesn't matter if some people in the population disagree with it, it's part of the curriculum. That's a really good step. Um, I think the Government can still work on educating people.. I mean so that's caught like my generation, maybe the generation behind me, and all the generations that follow on from me, but I think that we still need to catch people that haven't been through that limit? So I think education is something that's really really important..." (Participant 4, low SDO). 
Participant 11 similarly emphasizes the value in education about climate change:

EXTRACT 62: "I think any education that um aims to give people insight into how they can reduce their effects on the earth is really valuable, probably one of the most valuable tools for reducing pollution and that sort of thing." (Participant 11, high SDO).

While also acknowledging the importance of individuals having knowledge about climate change, Participant 13 criticized the portrayal of climate change as a debated topic:

EXTRACT 63: "But also climate change is not as discussed or when it is discussed it's put into some sort of crazy debate format where they're like 'is it really true?', I mean, five minutes from this scientist and five minutes with this guy who said it snowed in his yard last year, I mean it's not fair y'know there should be some kind of, everyone should be at least knowledgeable on these issues and then we can see what happens." (Participant 13, low SDO).

Indeed, in an effort to achieve 'balance', mainstream news reporting on climate change has historically framed it as a controversial issue. This is reinforced by the type of 'experts' sourced for stories on climate change, where politicians, business sources, and interest groups are chosen to voice their views rather than climate scientists (Brossard, Shanahan \& McComas, 2004; Trumbo, 1996). However, the extent to which the issue is framed as controversial or debated in NZ media is unclear. For instance, an analysis of New Zealand news media conducted over a decade ago suggested that fewer than ten percent of mainstream news stories frame climate change as a debate, and examination of the stories that do mention both 'sides' largely did so to discount climate change deniers (Kenix, 2008).

More recently, major online news outlet Stuff (stuff.co.nz, 2016) moved to ban climate change denial, stating that they reserve the right to delete reader's comments that deny climate change and adding the following disclaimer to articles on the topic: "Stuff accepts the overwhelming scientific consensus that climate change is real and caused by human activity. We welcome robust debate about the appropriate response to climate change, but do not intend to provide a venue for denialism or hoax advocacy." 


\section{"Infrastructure"}

The Infrastructure code includes extracts that discuss investing in, and improving, infrastructure as a way to mitigate climate change or encourage proenvironmental action. Infrastructure here includes public transport, recycling facilities, and greener sources of energy. Many of the participants who talked about these improvements to infrastructure suggested that people will choose these actions if they are better set up, or more accessible. For example, Participant 8 points out that public transport needs to improve in order to encourage people to do away with personal cars:

EXTRACT 64: “Like transport I don't, if the transport system is improved in Wellington and Auckland, I think it's quite good now but realistically you need to have a car, but if it was improved I would feel comfortable just using transport like I do now, public transport, not needing a car and um.. yeah I'm not sure what else." (Participant 8 , high SDO).

In the exchange below, Participant 33 (moderate SDO) also makes this point, but rather than the shortcomings most participants list about public transport (e.g. time consuming, unreliable), they have a different rationale:

\section{EXTRACT 65:}

Participant 33: "I think just cuz public transport is just so unpleasant, like it's literally disgusting and I hate it, it's the weirdest thing"

Interviewer: "Is that just in New Zealand"

Participant 33: "No that's like worldwide I'm telling you it's just the weirdest, it's just so gross, but then that's something the government could like improve on is just make public transport better and like more appealing to people because it's just, nobody wants to do it because they're like 'Ew, a bus' like [laughs] it's crazy"

Participant 23 had several suggestions for improvements to infrastructure that could see more people using public or active transport options, as well as renewable energy:

EXTRACT 66: "Um.. hmm um I think they should have, should be able to influence in some way how power is generated um y'know, giving or 
something to more environmentally responsible power sources or y'know saying 'We should change from this to that', um making more stuff like bike tracks, walking tracks, um more buses or something just more yeah, so you don't have to use personal transport I think as much as you do, especially in cities, and then in New Zealand it's like what five or six big cities, it's definitely something that would be easy to do." (Participant 23, low SDO).

Research on perceptions of transport options show that personal cars are rated more favourably than public transport along many dimensions, such as convenience, comfort, and status (Steg, 2003). Individual's awareness of climate change is only weakly associated with their transport decisions (Anable, Lane \& Kelay, 2006), thus it is likely that these other barriers, including public perception of public transport, may be better predictors of its use.

\section{"New Technology Will Save Us"}

Many participants appeared to hold the belief that advancements in technology will ultimately reduce the risk of climate change, either through mitigating the problem, or helping us adapt to a changed climate. Participant 1 recalled an invention they had heard about to turn pollution into jewelry, and later suggested that implementing more things like this would likely solve climate change:

EXTRACT 67: "I mean I know, what is it like, toxins are released into the air, I mean, they could find ways, cuz I know there was this new building in China that can like, sucks in all of the polluted air, and then it turns into jewelry? and now this new, this man's like making millions off of it, he's selling polluted jewelry, it's a bit weird." (Participant 1, high SDO).

Without naming any specific technology, Participant 56 suggested that advancements could reverse climate change:

EXTRACT 68: "Maybe I dunno in the future we might be more advanced that we can reverse the process?" (Participant 56, moderate SDO).

Participant 18 articulated the position that new technology will save us to justify sticking to the status quo. Specifically, they did not argue people ought to change their behaviour to mitigate climate change, because they argued that we are 
already on the right track. They refer to new technological advancements to back up this stance:

EXTRACT 69: “Just keep doing what we're doing right now, um ah employing other tactics in terms of transit, such as uh there was this um, there's this new car that runs on salt apparently, that with no greenhouse emissions, and there are hydrogen fuel cells um, pretty much the fuel of the future, and eventually we are going to run out of fossil fuels and petrol and that won't become a problem." (Participant 18, moderate SDO).

In the early 1990's, Wortmann, Stahlberg and Frey (1993; as cited in StollKleeman et al., 2001) identified that some people are confident that there are technological solutions to climate change, which related to engagement in proenvironmental behaviour. Stoll-Kleemann et al. (2001) again found evidence to support this position, where many members of their focus groups held a 'managerialfix interpretation' of climate change, which is the expectation that technology will solve the problem. Much like participants in my interview study, Stoll-Kleemann et al.'s participants made reference to advancements in renewable energy and electric cars (p. 114). Lorenzoni et al. (2007) similarly report reliance on technology as a solution for some.

Interestingly, in a rare example of a contradiction within a theme, one participant directly opposed this view. Participant 11 argued instead that new technology will not solve the problem, while highlighting some of the problems associated with the belief that it will:

EXTRACT 70: “I remember John Key I'm not quite sure I think last year he said something about, he was discussing climate change, and he said something along the lines he of um 'But we don't have to worry because New Zealand's brilliant and innovative science um scientists will come up with solutions to these issues', and I'm not, don't quote me on his quote, but um that kind of thinking is idiotic, it just pushes the responsibility to the science community to provide new technology that deal with the problems rather than actually decreasing the personal um, yeah it's just trying to get rid of the personal liability towards what how we actually everybody contributes towards this problem." (Participant 11, high SDO). 


\section{"Leading by Example"}

Many participants suggested that someone ought to encourage other people to act pro-environmentally, or show them how to act. Some participants, like Participant 39 , suggested that the government or business leaders should take the lead on climate change:

EXTRACT 71: "Yeah individuals will just do whatever individuals do, but if on a bigger front and there's more support and the government is saying or important corporations are saying 'Guys we need to sort out what we're doing to the environment' society's gonna follow and people are gonna follow." (Participant 39, moderate SDO).

Participant 40's extract is representative of those who argued that celebrities, world leaders, and others with influence ought to lead by example on climate change:

EXTRACT 72: "Everyone's gotta put in their effort but like world leaders like high up people need to sort of influence it like just at the Oscars back in February how Leonardo de Caprio said in his speech like, oh he at the end of his speech he was y'know thanking everyone and for his um movie the revenant said how they had to go to the southern tip of the Earth just to find snow to shoot it like climate change is real like we needa not take this like planet for granted and I think a lot of people seeing that and seeing like someone they idolize like that is y'know they're gonna pay more attention, and it's just sorta those people using their like influence or like their position in the world to like.. make, bring attention to issues like that, I think that really does help." (Participant 40, moderate SDO).

Participant 14 (high SDO) divulged that they did not always believe in climate change, explaining how information about climate change from trusted sources changed their mind:

\section{EXTRACT 73:}

Interviewer: "What changed your mind?"

Participant 14: “Just exposure I guess to information, you kinda y'know, you can't really deny it, especially when you hear it coming from sources that you 
trust so I mean y'know this is just on an individual level but y'know when you look up to certain people and when all these people say it's really happening then you really go y'know, they're right about most things, they're probably not gonna be wrong about this."

Leading by example harnesses the power of social influence as a source of change. Indeed, social influence approaches, such as block leaders (individuals who spread awareness about an issue) and modeling (exhibiting pro-environmental behaviour for others to copy), do tend to motivate pro-environmental action (Abrahamse \& Steg, 2013).

\section{“Global Agreements”}

Another top-down solution was a greater emphasis on global agreements, such as the 1997 Kyoto protocol and the more recent 2015 Paris Agreement. While some participants expressed the belief that global agreements are needed (e.g. extract 74), others listed issues with such agreements (e.g. extracts 75 and 76):

EXTRACT 74: "Um first of all it is not just a single countries effort, it's global issue, um... I think governments or associations, organizations form every single country, they should increase their communication, and yeah solve this human being thing, we should solve it together." (Participant 19, high SDO).

EXTRACT 75: "From memory based on laws lectures I think, the UN has agreed, well individual countries in the UN are saying 'Right well we'll help join this fight against climate change, we'll do something, but we're not doing it first', so basically it's a weird stand off situation where everyone's willing to act, but no one wants to do it first, I think that's probably a big problem." (Participant 15, high SDO).

EXTRACT 76: "I think they had those Paris those talks or whatever a little while ago where they actually were like 'Okay, we're gonna institute these before twenty twenty five' and I think a lot of people signed, and I think that's a positive step but kind of gotta wait and see if they're actually gonna do it, because obviously governments change and yeah there's instability and things 
like that y'know if certain people get into power somewhere they might y'know, reverse those kind of talks." (Participant 45, moderate SDO).

I interpreted both suggestions about global agreements and criticisms of past agreements as fitting the Global Agreements code, given that criticisms implied a belief in the importance of upholding such agreements. It is important to note that the interviews occurred before Trump was elected into office (subsequently positioning the US to exit the Paris Agreement), and therefore Participant 45's extract is somewhat prescient.

\section{"Incentives"}

The last top-down solution mentioned in the interviews was Incentives. These included incentives offered to those engaging in pro-environmental actions, and were almost exclusively monetary incentives, such as tax cuts and discounts.

Many participants suggested that the government should offer businesses financial incentives to encourage pro-environmental action:

EXTRACT 77: "I think the government should have some type of way of... not forcing companies to do their part, but encouraging, maybe having some incentive to want to help out somehow like to put money towards like, government still has finite money like they they can't obviously pay for anything, and no matter what they do someone's gonna be complaining about them not doing something else, if not the planet they' $d$ be complaining about housing and stuff.. but like if they could find a way to get those companies on board to help them pay for some stuff." (Participant 28, moderate SDO).

Others made similar suggestions for encouraging individuals to act, such as the extract below:

EXTRACT 78: "Oh the whole car, fuel emissions reducing them, and so just incentivizing people not to just use their car when they feel like it and tryna do other stuff." (Participant 9, low SDO).

Finally, some participants suggested less direct ways to incentivize proenvironmental action. For instance, Participant 59 referenced the inbuilt incentives for businesses that would not require government intervention: 
EXTRACT 79: "Um there's a big thing say with factories and that kind of thing, tryna.. tryna look at exactly how much they're y'know releasing into the environment, and that kind of thing, there is more of a duty there in terms of if you're part of the problem, you've gotta, you should be looking at how you can contribute towards a solution, I mean even just economic standards it's good PR." (Participant 59, moderate SDO).

Research on incentives indicates that they can be effective in shaping behaviour in the short term, though people often revert back to past behaviour once the incentive is removed, as the underlying motivation for action is to get the reward (Gneezy, Meier \& Rey-Biel, 2011). With the financial incentive remaining in place, change can be sustained. As one example, in Massachusetts less than $10 \%$ of recyclable materials overall are recycled, however compliance with recycling bottles is as high as $80 \%$ as these can be returned for a five-cent refund (Kollmuss \& Agyeman, 2002). Incentives may also be social (such as gaining other's approval), which tend to be more effective than monetary rewards (Gneezy et al., 2011).

\section{Bottom-Up Solutions}

\section{"Little Things"}

Just over half of participants talked about taking small actions, and doing "little things", to do their part for the planet. The three extracts below are good illustrations of this code:

EXTRACT 80: "When I have the ability to be able to pay more for stuff that's better for the environment like just little things, I will.” (Participant 28, moderate SDO).

EXTRACT 81: “Um.. even just like one person just like recycling or like maybe biking more instead of using cars, or even the smallest little thing is like, obviously you can take it to the next level but like informing others, making groups, like getting the word out there and yeah even like you could try and take it like petitions and all that kind of stuff can also affect how people see the world like if you get enough attention things can change, so yeah." (Participant 38, moderate SDO). 
EXTRACT 82: "Yup um just like the most simplest things that even some people take for granted you know, as simple as recycling bin, rubbish bin, paper." (Participant 36, moderate SDO).

Many participants used the exact phrasing "little things": In fact, an NVivo search returned a total of 30 uses from 16 participants using the exact phrasing "little things", and a further seven included "small things".

\section{"Collective Action"}

Here, I use the term collective action to gather extracts that mention everyone doing their part, and working together to mitigate climate change. Many participants argued that working together on climate change is likely to be the most effective way of addressing the issue (sometimes explicitly contrasting this with individuals acting in isolation, as in the Self-Efficacy code):

EXTRACT 83: "I guess it can really be taken up by any individual, any individual can make a change, and saying that like we should leave it to the government and let them sort it out isn't really being an active member of society, whereas like if we all worked together to do this then it could actually happen, like sure the government is like this big thing, but like one person can make a difference, and if they got enough people together then it could like, it may be a slow like gradual change, but it would do something." (Participant 38 , moderate SDO).

Other participants emphasized that we all need to work together to solve climate change:

EXTRACT 84: “Anybody who um uses um electricity and drives cars are responsible for contributing, even if it's just a small personal amount it all adds up, everybody's responsible for that, so basically probably anybody but Amish communities or people who are [laughing] completely off the grid." (Participant 11, high SDO).

EXTRACT 85: "We're all living here at the moment, we're all living on earth, it's everyone's job to work together." (Participant 51, high SDO). 
This code relates to the idea of collective efficacy, defined as "the belief that one's group is capable of affecting relevant aspects of its environment" (Reese \& Junge, 2017, p. 200). Reese and Junge (2017) demonstrated that endorsing higher levels of collective efficacy is associated with greater intentions to engage in sustainable behaviours. Therefore, the argument for collective action in this code could be motivating. The key with this code is that the responsibility for acting is collective, so this could also be an expression of diffusion of responsibility, which may instead stifle intentions to act. This relates back to the Self-Efficacy code discussed earlier, where individuals perceive their own impact as limited.

\section{“Co-benefits"}

In this code, I included all mentions of engaging in pro-environmental behaviours that were not purely motivated by pro-environmental intentions. For instance, participants often reported getting other benefits out of acting proenvironmentally. This code was placed into the Bottom-Up solutions subtheme because the ideas shared are participant's own justifications for engaging in individual action, rather than necessarily benefits that are introduced from the top-down, as in the Incentives code. Below are three examples of this code, demonstrating (respectively) references to saving time, money and the novelty of a proenvironmental action, and a personal benefit:

EXTRACT 86: “If it's realistic, like I know I won't be in rush hour so, twenty minutes compared to an hour on the highway, if it was an hour I would definitely take the train." (Participant 24, high SDO).

\section{EXTRACT 87:}

Participant 34: " I went home over the holidays and I bussed up instead of flying, and it was a pretty big, that was a pretty big, like north of Auckland so I bussed for like over, I was bussing all day, it's like thirteen hours to get from Wellington to Whangarei, and then like parents picked me up from Whangarei” Interviewer: "And so why did you decide to do..." 
Participant 34: "I mean mostly because it was cheaper, and it was like a new experience, I was like 'you know, I can take a bag with me, it doesn't cost extra like it does on a plane, it could be a fun experience'.” (Low SDO)

EXTRACT 88: "Um.. well I choose to instead of turning on the heater just putting on more clothes and wrapping up with blankets before doing that just to save on power and gas consumption that way, just because it's also better for your skin because it's not as drying on your skin, and I've got really dry skin already, so it's like I might try to sacrifice the instantaneous heat for being able to have the sort of not as terrible skin." (Participant 57, moderate SDO).

It is unclear if, in these instances, behavior is being shaped by proenvironmental intentions. Many of the extracts in the Co-benefits code appeared to explain taking certain actions, and retrospectively evaluating them as good for the environment (e.g., taking public transport), while the original motivation was not proenvironmental (e.g., to save time). In the case where the co-benefit motivates the behaviour, this might relate more broadly to ideas of Incentives discussed above.

\section{"Food Choices"}

In the course of analysis, I was surprised to find that a large number of participants acknowledged the association between animal agriculture and climate change. This was surprising because previous research suggests that individuals are not aware of this link (e.g. Macdiarmid, Douglas \& Campbell, 2016). Abstaining from eating meat is also relatively less common among New Zealanders and those in my sample, with an item on diet in the preselection data revealing that only six of the 59 participants in my sample identifying as vegan or vegetarian. Despite this, many participants put forward cutting down on meat and other animal products as a potential solution:

EXTRACT 89: “Um like I said it's pollution, I think it's also there's studies who, I'm not um I haven't studied it, I haven't researched it, I've just seen what I've seen on the internet I suppose, um I think that like cows or something produce the most amount of methane and that's like the biggest contributor, more than cars, and so I dunno maybe that's another thing that could be changed, is if everyone doesn't go vegan but I mean we're at the time 
where we don't really need to be growing and slaughtering cows, I mean we could be coming up with some sort of scientific product on the side that's like meat but not actually meat and we can stop all these cows from farting and ruining the atmosphere." (Participant 13, low SDO).

As well as recognizing the impact of animal agriculture on the environment, Participant 14 suggested lab-grown meat as an alternative:

EXTRACT 90: "I know it seems like something coming up in the next I dunno twenty, twenty five years is gonna be um sorta synthesized meat or artificially made meat so I mean, when that comes in that'll kind of eradicate the problem of the dairy industry, anyway well meat industry, cuz I mean you don't really need to grow cows when you can make it in a lab for cheaper, um so I guess sort of just heading in that direction, um although that's not gonna be the best for the economy, seeing as we rely heavily on dairy, I think that could almost help out because I know that especially the meat industry contributes a lot to pollution in our country." (Participant 14, high SDO). In the following exchange, Participant 30 (high SDO) alludes to people ignoring the link between animal agriculture and climate change:

\section{EXTRACT 91:}

Participant 30: "Um, well really the only solution, and the thing nobody wants to do, is to change, a change of lifestyle right across the board, so like less consumption of meat or less farming sorta thing, um but no one is really willing to do that, everyone wants like the nice ending but no one wants to really change anything."

Interviewer: "Why do you think that is?"

Participant 30: "Well, when it comes back to the sorta like meat thing, no one really is willing to admit that the meat industry causes a lot of problems or anything. And people are just happier to eat meat and continue living like that despite it being like not really.. proven to not necessarily be great for the environment, but because it's sort of an enjoyable thing to do like people like doing it." 
Research is consistently demonstrating that even the lowest-emission animalbased foods regularly surpass plant-based alternatives in terms of greenhouse gas emissions (Poore \& Nemecek, 2018), with the typical western diet emitting about twice the greenhouse gasses of plant-based diets (Scarborough et al., 2014). At a country level, the environmental impact of animal agriculture is a serious concern for NZ (Parliamentary Commissioner for the Environment, 2016). Because of the size of NZ's agricultural industry, agricultural emissions account for a disproportionately large amount of our total greenhouse gas emissions (Clark, Kelliher \& Pinares-Patino, 2011). This is a unique emissions profile when compared to other developed countries, thus presenting a unique challenge for NZ.

\section{"Public Pressure"}

This code includes extracts that discuss encouraging business and government (i.e. 'top-down' forces) to take action. This is included under the 'bottom-up' solutions subtheme because participants describe individual 'grass roots' actions taken with the aim of motivating further top-down action. This could be achieved through petitions, boycotts, or voting for change. Participant 16 argued that people ought to raise the issue by lobbying those in government:

EXTRACT 92: "I think just telling whoever is in government, your local MP or y'know the future Prime Minister or whatever that y'know you care, and you want a future for your children and you want a future for yourself." (Participant 16, low SDO).

Similarly, Participant 11 suggests lobbying or protesting to spread awareness of climate change:

EXTRACT 93: "You can join a lobby group [laughing] or march on the capital I think, um yeah just public, any public pressure towards that issue would um yeah, just bringing it more to the media's attention, everyone's attention, would help address the issue." (Participant 11, high SDO).

Extract 93 was also allocated to the Awareness and Education code, as it relates to the idea of educating and informing others. In an alternative suggestion that similarly involved public pressure, Participant 14 suggested boycotting polluting industries: 
EXTRACT 94: "Um I mean.. it yeah surely I mean obviously when we, I dunno buy from companies or um, we're kind of saying 'Well, what you're doing's okay', so, and it's kind of hard with dairy because you can't really just, well you can stop buying meat or whatever or milk, but I mean y'know that seems a bit unreasonable but I mean there's certain industries I guess that which I'm unaware of that probably y'know pollute and that kind of thing just not kind of buying the products or whatever is gonna send a message to them." (Participant 14, high SDO).

\section{"Make things a habit"}

Some participants talked about environmental behaviours becoming easier and more automatic once they become habitual, and thus advocated for lifestyle changes as solutions to climate change. For instance, Participant 13 talks about how changes might initially be restricting, but eventually become habitual:

EXTRACT 95: "So I stopped driving, because I came to Wellington and there's just not much point driving here, I stopped obviously using petrol and then I've ceased eating meat recently and it's just.. once these things start to happen and you put these restrictions on yourself and then they no longer become restrictions, just become your way of living, it's not really a sacrifice or a change." (Participant 13, low SDO).

Others advocated for environmentalism to be taught to children, again relating to the Awareness and Education code discussed earlier, so that people grow up behaving pro-environmentally, and these behaviours are ingrained as habits:

EXTRACT 96: “And that's the sort of thing that's let's say, I was taught at a young age, and if we teach people about the environment, how we should take care, how we should treat one another, this thing would just come naturally, not, it will come naturally." (Participant 3, high SDO).

Participant 50 (moderate SDO) took this a step further to place responsibility on caregivers to introduce pro-environmental behaviours, which might then continue: 


\section{EXTRACT 97:}

Participant 50: "Back at home I know that we always had to have showers three to five minutes, we all made sure that we had to do that, and um" Interviewer: "Is that like, family?"

Participant 50: "Family, yeah so being brought up with it I suppose it carries on, so now that I've left home I still try to make sure that I follow that stuff so yeah a lot of responsibility is on caregivers, um yeah." (Participant 50).

In contrast, because habitual environmentally harmful behaviours are difficult to shift, Gifford (2011) identified habit (or 'behavioural momentum') as one of the 'dragons of inaction' on climate change. Some habits are entrenched in our daily lives, and these are resistant to change, though not impossible (Gifford, 2011). Making pro-environmental behaviour habitual and, therefore also resistant to change, could be a good avenue for future research.

\section{Exploratory analyses on the relation of SDO}

The analyses so far have looked for patterns (themes and codes) across all 59 interviews. In this section I describe some exploratory analyses, where I coded for the presence or absence of each code for each participant, and looked for differences across SDO-level.

I conducted each analysis twice: firstly with SDO indexed by group membership (low-, moderate- and high-SDO), and then using the mean level of SDO. When SDO was indexed by group membership, I conducted a Chi-square test, and report this with the Phi measure of the degree of association. These values were interpreted according to Rea and Parker's (1992, p. 203) guidelines: up to $.10=$ negligible association, $.10-.20=$ weak, $.20-.40=$ moderate, $.40-.60=$ relatively strong association, $.60-.80$ strong association, .80 and above $=$ very strong association. When mean level of SDO was used, I report the point-biserial correlation, and interpret the strength using Cohen's (1988) guidelines.

These results are summarized in Table 5.6. Of the 31 individual codes, only one had a significant association with SDO (i.e. $p<.05$ ), and only when this was indexed as SDO-group (i.e. the Chi-square tests). The significant finding disappears 
when controlling for multiple comparisons and the associated increase in familywise error rates. 
Table 5.6.

Associations between SDO and the codes developed in Study 4.

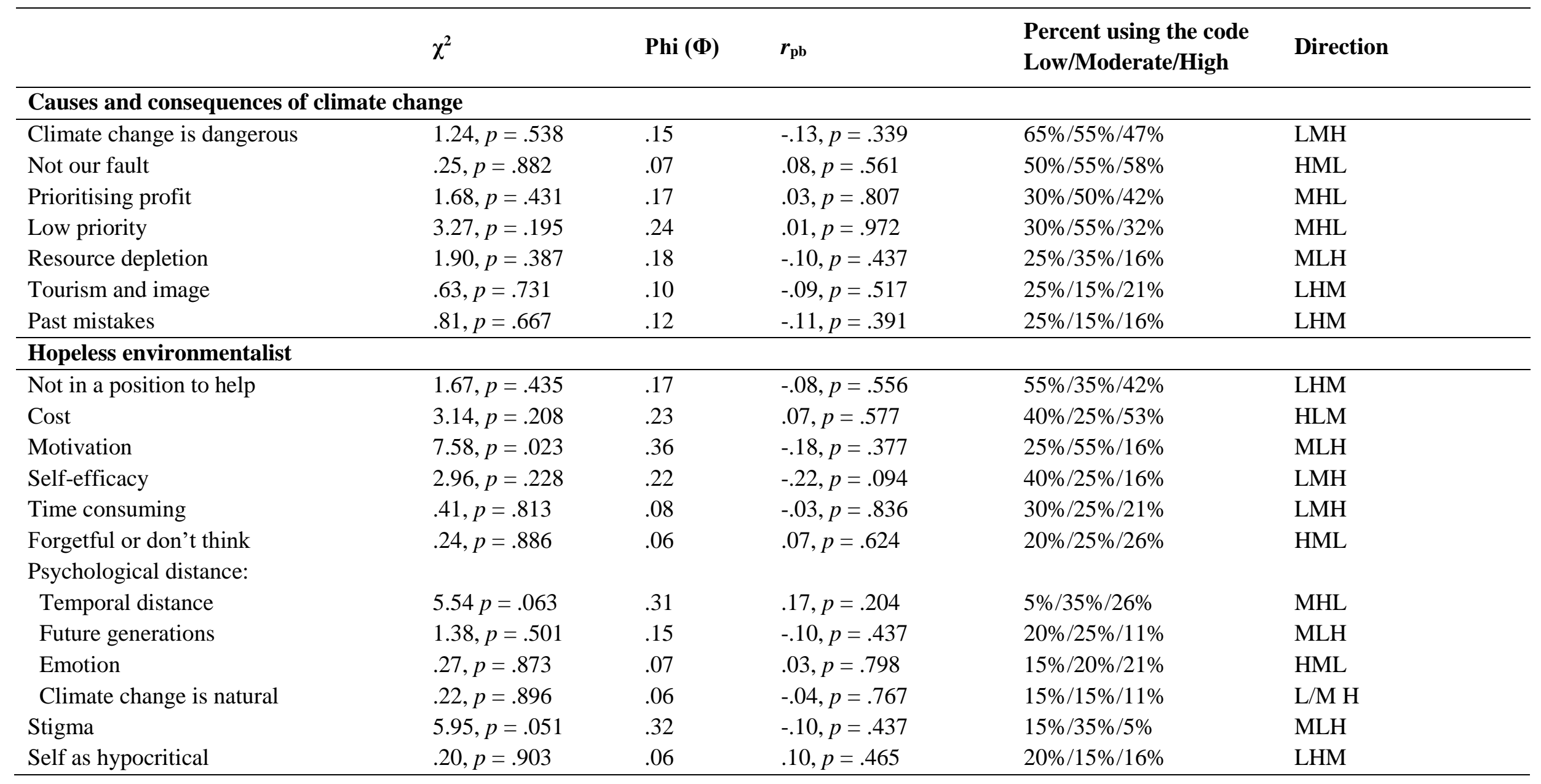




\begin{tabular}{|c|c|c|c|c|c|}
\hline \multicolumn{6}{|l|}{ Top-down solutions } \\
\hline Regulations & $.83, p=.661$ & .12 & $-.11, p=.425$ & $65 \% / 65 \% / 53 \%$ & $\mathrm{~L} / \mathrm{M} \mathrm{H}$ \\
\hline Awareness and education & $3.75, p=.153$ & .25 & $.13, p=.332$ & $45 \% / 75 \% / 58 \%$ & MHL \\
\hline Infrastructure & $.44, p=.804$ & .09 & $.03, p=.813$ & $45 \% / 55 \% / 47 \%$ & MHL \\
\hline New technology will save us & $2.13, p=.345$ & .19 & $.09, p=.492$ & $35 \% / 25 \% / 47 \%$ & HLM \\
\hline Leading by example & $1.37, p=.504$ & .15 & $-.17, p=.194$ & $30 \% / 30 \% / 16 \%$ & $\mathrm{~L} / \mathrm{M} \mathrm{H}$ \\
\hline Global agreements & $2.28, p=.319$ & .20 & $-.21, p=.117$ & $30 \% / 20 \% / 11 \%$ & LMH \\
\hline Incentives & $.92, p=.631$ & .13 & $.10, p=.435$ & $15 \% / 10 \% / 21 \%$ & HLM \\
\hline \multicolumn{6}{|l|}{ Bottom-up solutions } \\
\hline Little things & $1.33, p=.516$ & .15 & $-.16, p=.219$ & $60 \% / 55 \% / 42 \%$ & LMH \\
\hline Collective action & $.72, p=.697$ & .11 & $.17, p=.207$ & $45 \% / 55 \% / 58 \%$ & HML \\
\hline Cobenefits & $1.24, p=.538$ & .15 & $.08, p=.551$ & $45 \% / 35 \% / 53 \%$ & HLM \\
\hline Food choices & $3.95, p=.139$ & .26 & $-.08, p=.544$ & $50 \% / 20 \% / 37 \%$ & LHM \\
\hline Public pressure & $2.48, p=.290$ & .21 & $.09, p=.523$ & $30 \% / 15 \% / 37 \%$ & HLM \\
\hline
\end{tabular}

Note. Directions are in order from most to least common use of the code $(\mathrm{L}=$ low-SDO group, $\mathrm{M}=$ moderate, $\mathrm{H}=$ high. None $=$ no directional prediction). 
The lack of significant results from these exploratory analyses is likely at least in part due to the small sample size. I did not have sufficient statistical power to detect small effect sizes, as the data came from only 59 participants in total. The effect sizes were mostly small (based on examinations of the Phi and the point-biserial correlations), with the highest effect size of .36 corresponding to a moderate effect size. Using $2 \times 3$ contingency tables (binary absence/presence of codes across the three SDO-level groups, with two degrees of freedom), power analyses reveal that a sample size of 964 participants is required to detect a small effect size of .10. These requirements reduce to 107 participants to detect a medium effect size (.30), and 39 participants for a large effect size (.50). In my study, only associations with mediumto-large effect sizes reached statistical significance (before controlling for repeated comparisons). Therefore, these findings are broadly consistent with a low power interpretation.

\section{Discussion}

In this study, I examined how individuals talk about environmental issues by analysing the content of 59 semi-structured interviews. Exploratory analyses of how the ideas expressed in the interviews might relate to participant's endorsement of SDO did not return any significant results. However, through the process of thematic analysis I highlighted some important findings of laypeople's ideas on the environment and climate change, which I use in the next chapter for a more precise and larger-scale examination of whether and how they relate to SDO. While my discussion of the codes and themes was interwoven into the analysis above, I summarize and expand on some key points here.

I identified that when prompted to discuss climate change, students respond by citing the causes and consequences of the problem. Most participants described climate change as having potentially devastating effects. Despite this, and perhaps in an attempt to put distance between themselves and the issue, many participants also deflected blame for climate change by arguing that the problem is causally linked to the actions of profit-hungry corporations and mistakes individuals have made in the past, with some students even rejecting the idea that humans contribute at all.

This suggests that even with the best intentions, people may aptly be named Hopeless Environmentalists, as personal barriers prevent them from taking action to 
mitigate climate change. These barriers include the perceived costs, effort, time, and stigma associated with mitigating action. Some participants said they would like to act, but pro-environmental action is out of their control, or that their actions at an individual level would do little to achieve change. Action to address climate change is framed as a delicate balance between competing interests, with environmentalism losing the war of priorities (instead opting against spending time, money, and effort on environmental causes). Therefore, while a majority of participants expressed concern over the potential devastation to come from a changed climate, almost all also listed some barriers that excuse their own inaction.

These barriers broadly map onto many of the types of impediments identified in Lorenzoni et al. (2007) and Stoll-Kleemann et al.'s (2001) qualitative research and are generally supported by the wider environmental psychology literature. The barriers appear to function as a way of alleviating personal responsibility and guilt associated with inaction (i.e. cognitive dissonance; Festinger, 1957), while also helping to justify and maintain the status quo: a participant's current (perhaps environmentally exploitative) way of life. They exist perhaps because inaction on climate change is extraordinarily beneficial at the individual level. This is something that many participants identified: acting may require sacrifices to financial wellbeing, time, energy, and even reputation when actions are associated with stigma. When a critical mass of people engage in environmentally harmful behaviours and forgo mitigating action, the planet cannot cope with the demands for resources or greenhouse gasses emitted, and this has culminated in the current crisis (IPCC, 2007; 2014). This is a true tragedy-of-the-commons scenario, and Gardner and Stern (1996) further point out that individuals do not recognize their own inaction as problematic, given that their impact is so small in the context of the global resources being exploited.

Participants acknowledged that this tragedy occurs at a number of levels. At the individual level, participants struggled to see the effects of their actions: both positive (Self-Efficacy) and negative (Not Our Fault). Specifically, many participants agreed that individual pro-environmental actions barely contribute to solving the problem, while at the same time individual's environmentally harmful actions are perceived as barely contributing to causing the problem. Participants suggested that business-level decisions make a greater impact and are made in favour of profits 
regardless of the impact (Prioritising Profits), constructing industries as unwilling to cooperate and instead choosing environmentally exploitative practices. At the country-level, participants understood the difficulty in honouring global agreements when the effect of doing so is detrimental to one's nation, and the resistance to doing so when other countries fail to hold their end of the bargain (Global Agreements). Furthermore, a few participants also placed themselves between previous generations that they perceived as to blame for climate change (Past Mistakes) and those we ought to preserve the environment for (Future Generations). Climate change is therefore an issue characterized by competing interests (and impacts) at the individual-, businesses-, country-, and generational-level.

Participants often recognized these tensions, for instance by explaining the trade-off between economic and environmental concerns and the balance between competing values and priorities. This highlights the trade-offs required when making the decision between pro- and anti-environmental behaviours. Indeed, people tend to struggle making decisions where there are competing values at play. Tetlock, Lupia, McCubbins and Popkin (2000) explain that part of this difficulty comes from the fact that different values cannot be put on the same metric - to what extent are economic losses acceptable in order to lessen the impact on the environment? This is a big question, but people regularly make many decisions of this kind. For instance, is it worth sacrificing an extra five dollars in order to purchase a more environmentally friendly option at the supermarket? This is an example that one participant used to excuse their own environmental inaction. In this case, it is difficult to gauge how much damage choosing the cheaper, less environmentally friendly option would cause, and if this warrants paying the extra cost. Nevertheless, the effects on the environment and on one's finances are not directly comparable.

Once a decision between competing values is made, Tetlock et al. (2000) argue that people struggle to reconcile rejecting one of their values - say, if they chose economic value through rejecting the environmentally friendly option - and will downplay their investment in environmentalism in an effort to maintain consistency. Tetlock et al. point out that humans have always faced constraints on their resources, time and energy. We can only do so much, and therefore being faced with decisions where our values conflict is inevitable. But what happens if the values relevant to a situation are not salient at the time (as evidenced under the Forgetful or Don't Think 
code)? For instance, comfort or taste preference might 'win' the decision of what to buy or eat simply because the environment is less salient at the time of choosing. In this case, people are not carefully weighing the costs and benefits of different options, and the environmental consequences of day-to-day behaviours may not even factor into the decision.

Each of the barriers identified from the interviews help explain the divide between pro-environmental attitudes and environmental inaction. While many participants easily accounted for their own inaction, they also readily shared their ideas about how to solve the issue. The first type of solutions involved individuals taking action: by working together on the issue, putting pressure on governments and businesses to effect change, forging new habits, changing their diet, taking actions that benefit them in other ways, and doing Little Things that might help. While these are all commonsense, it is unclear how many participants actually engage in these actions (especially given the multitude of barriers in their way that they themselves identified).

If the frequency with which the suggested solutions were cited is anything to go by, action 'from above' by way of government regulations might be a palatable solution. However, there are still barriers to public acceptance of such regulation. In one example, the debate surrounding including greenhouse gasses associated with animal agriculture in the emissions trading scheme in NZ took a turn for the worse when this was labeled a "fart tax" (Driver, Parsons \& Fisher, 2018). Furthermore, even if regulation is introduced to change behavior, it is not always able to change people's values (Lorenzoni et al., 2007).

Where a genuine institutional barrier was identified, such as the lack of facilities to recycle or public transport options in rural areas (Not in a Position to Help), this was mirrored by suggestions aimed at facilitating these options (Infrastructure) as a solution. This is a good recommendation as long as putting systems in place to enable these behaviours is enough to spur action. However, it is likely that work might also need to be done to make these options more attractive (e.g. in the case of buses being less 'sexy' than cars: Steg, 2003).

In essence, while participants could readily list a range of viable options for addressing climate change, the discussions about climate change were heavily 
speckled with excuses. In fact, many of the solutions have an accompanying 'get out of jail free' card, where participants put forward a solution but were unable or unwilling to engage with the solution for several of the reasons falling under the Hopeless Environmentalist theme. Therefore, my research reconfirms the need to focus efforts on changing the attitudes and values that underpin (in)action on climate change, as well as continuing to find ways to encourage people to act in alliance with such values, in order to skew trade-off decisions in favour of environmentalist concerns.

This will involve a deeper exploration of the psychological barriers to proenvironmental attitudes and action. In my thesis, I try to improve understanding of how one barrier in particular, social dominance orientation, relates to environmental attitudes and action. However, an initial examination of how this construct relates to the ideas presented in the interview study did not detect any differences across SDOlevel. If it really is the case that SDO is unrelated to the frequency that codes are used (a possible finding I approach with caution), then this may indicate that individuals relatively higher in SDO are just as aware of possible causes, consequences, solutions and barriers to action towards climate change as their low-SDO counterparts. It might just be the case that these ideas do not move them to engage with the issue. This is consistent with endorsement of SDO predicting greater tolerance of actions that harm others (Wilson, 2003). Perhaps they identify the same arguments as their pro-equality counterparts, but these matter less to them or have little effect on their preference for environmental exploitation, as the ends justify the means.

In many cases, the prevalence of the use of codes are in the expected directions. For instance, Climate Change is Dangerous is cited most often by individuals low in SDO consistent with quantitative research linking SDO to lower anxiety about climate change, as summarized in Chapter 2. High-SDOs are least likely to suggest Regulation and Global Agreements as solutions, consistent with the conservative position against government intervention on climate change (Peterson et al., 1993), and more likely to deflect blame (Not Our Fault), akin to denial of responsibility found again among higher scorers on SDO (Vail \& Motyl, 2010). While none of these differences were statistically significant in this study, they warrant follow up with a larger dataset to overcome the issue of low power. 
Another limitation of this work is that the 'High-SDO' group is not a group of people who are oriented towards social dominance. Instead, the group on average neither agrees nor disagrees to items concerning inequality and intergroup dominance. The group of participants scoring in the upper quartile on SDO actually scored, on average, at the midpoint of the 7-point Likert scale, which is interpreted as expressing neither agreement nor disagreement. Therefore, while the group was high in SDO relative to the other two groups, they were not high in the sense that they (on average) agreed with the items on the scale. This affects the way these data are interpreted: instead of meaning that individuals who endorse (as in, agree or strongly agree with) social inequality and intergroup dominance, these participants are ambivalent towards it.

A further limitation to acknowledge here is that I did not have the resources to recruit a second coder to verify my allocation of codes using the coding scheme. I therefore am unable to check the reliability of these codes. This could have contributed to the lower associations with SDO than expected, as reliability is associated with the strength of an association, with less reliable measures tending to underestimate associations (e.g. Hunter \& Schmidt, 1991). Braun and Clarke (2013) warn against having codes checked and inter-rater reliability computed, as they subscribe to the position that qualitative research is inherently subjective. I agree with this position, though did take steps to reduce my influence on the data and improve objectivity, consistent with a postpositivist position. As part of this, I did have one section of the data (the discussions on deep sea drilling, not included in my thesis due to space constraints) independently coded. Using Cohen's Kappa to control for agreement achieved by chance, our coding aligned well, with reliability coefficients corresponding to moderate to perfect agreement for all codes.

The associations may also have been low because I recruited participants from a student population, where the association between environmentalism and SDO tends to be weaker than in general population samples (Chapter 2). This was in part an unfortunate consequence of the order that I did the research in this thesis: I started by collecting data for Study 4, and the meta-analyses were of the final pieces of work I did, although I presented them first in my thesis as this order was most sensible. This meant that I was unaware of the moderating effect of sample type when I chose to interview student samples, hence a limitation of this study. Chapter 2 showed that 
SDO relates to the same range of environment-relevant variables in student and general population samples. Therefore it is unlikely that, if interviewed, a general population sample might have different ideas on the environment than students, however their ideas might be more strongly tied to their ideology.

Despite the relative weaknesses of this work, the interview study provided useful insight into the ideas that students have about the causes and consequences of climate change, as well as potential solutions and barriers to mitigating the problem. It also utilized a large sample size for thematic analysis (Braun \& Clarke, 2014; Sandelowski, 1995). However, the exploratory quantitative analyses were underpowered, which prevented a thorough examination of whether there are differences in the extent to which high- and low-SDO individuals endorse these ideas. These findings have applied value for future work, as the ideas expressed in the interviews can be transformed into survey items to gauge the attitudes of a larger group of people, which is my aim in Chapter 6 . 


\section{Chapter 6}

\section{Understanding how student's ideas about climate change relate to endorsement of SDO}

\section{Introduction to Studies 5A and 5B}

In the previous chapter, I explored responses to open-ended interview questions about climate change. The purpose of this research was to better understand lay peoples' ideas on climate change, unrestricted by the closed-response formats of typical survey-based research in this area. I identified a number of key ideas (themes) that repeated across the interviews and broadly fit into three main categories: Causes and Consequences of Climate Change, Hopeless Environmentalist, and Solutions.

My sample size was limited to 59 participants, and although this is a large sample for qualitative research (Braun \& Clarke, 2014; Sandelowski, 1995), it is questionable as to whether the ideas identified from the interviews apply to larger group. This is a common criticism of qualitative research, as the aim is typically to describe the experience of those interviewed, accepting difficulties generalizing further. Another consequence of the small sample size is that preliminary analyses designed to test whether and how participant's ideas were associated with SDO were underpowered.

Many of the codes interpreted from the interviews warrant further examination in relation to the role of SDO in environmental attitudes and behaviours. In this Chapter, I develop survey items to gauge support for similar ideas as those expressed in the codes from Study 4. Based on theoretical grounds, I expect endorsement of some of these items to be associated with lower levels of SDO. For example, given the link between SDO and climate change anxiety (see Chapter 2), I would expect SDO to be negatively associated with agreement on items based on the Climate Change is Dangerous code. Items implying that our current use of the environment is exploitative (e.g. based on Resource Depletion, Prioritising Profit, Future Generations, Emotion) might also be favoured by low-SDOs, given that higher SDO scores are related to a preference for utilization of nature (Amerigo et al., 2016;

Hoffarth \& Hodson, 2016; Milfont et al., 2013). The endorsement of personal action on 
climate change (e.g. as in Habit, Public Pressure, Little Things, Food Choices, Collective Action) are also likely to exhibit a negative relationship with SDO (e.g. see relationship with support for action category of meta-analyses in Chapter 2). Finally, solutions involving public spending and involvement to regulate emissions (Regulations, Infrastructure, Leading by Example, Global Agreements) are likely negatively associated given the high-SDO preference against Green political parties and government involvement in regulating emissions (Sibley, 2009-2015). Consistent with their lower concern about climate change, and lower endorsement of environmentalism as an important value (e.g. Dimdins et al., 2016; Sibley, 2009; 2015), higher scores on SDO might be positively associated with endorsement of Low Priority and Forgetful or Don’t Think.

In contrast, some ideas are more likely positively associated with SDO. For instance, aligning with findings that SDO is associated with less willingness to make sacrifices for the environment (e.g. Study 2B), the Motivation, Cost, and Time Consuming barriers to action might be more common among high-SDOs. Consistent with the deflection of personal responsibility (Vail \& Motyl, 2010), Social Dominants might also be more likely to support the idea that New Technology Will Save Us. Individuals higher in SDO tend to be less likely to accept that humans are causing climate change (e.g. Devine-Wright et al., 2015; Milfont et al., 2013; Stevenson, Peterson, Bondell, Moore, \& Carrier, 2014), thus SDO might relate positively to the idea that Climate Change is Natural. Finally, Cantal, Milfont, Wilson and Gouveia (2015) showed that SDO relates to disdain towards dissident and derogated groups, including environmentalists. This suggests that high-SDOs see environmentalists as a group that is disliked, and therefore may be more likely to attach Stigma to pro-environmental actions.

For some codes, there are competing indicators as to the possible role of SDO. For instance, both Not Our Fault and Past Mistakes are characterized by deflecting responsibility for climate change (onto industry and previous generations, respectively). The deflection elements of these codes are consistent with SDO's association with lower assumption of responsibility for climate change (Vail \& Motyl, 2010). However, when the blame is placed on industry, SDO might follow the same pattern as RWA in opposing punishment for companies for environmental damage (e.g. Peterson et al., 1993). Blame is also deflected onto industry in the Self-Efficacy code, thus might align with lower endorsement of SDO. Similarly, for the Incentives code, there are reasons why Social Dominants might support such policies (as they are hierarchy-enhancing: the benefits go to those of higher status) or 
oppose them (possibly funded by taxes, thus involving a perceived personal cost). Tourism and Image centred on concerns that climate change will affect NZ's image and the natural attractions that boost our economy through tourism. This could be consistent either with a view of utilizing the environment (i.e. for economic gain), and therefore more often cited by individuals high in SDO, or alternatively a proxy for one's enjoyment of nature and desire to share that with others, and thus more strongly endorsed by individuals lower in SDO. Similarly, high-SDOs might endorse Co-benefits for selfish reasons. To support the opposite pattern of results, low-SDOs are more encouraging of pro-environmental action in general (e.g. Chapter 2) and this could extend to taking actions with co-benefits.

In this chapter, I return to quantitative methods to test whether a larger group of participants endorse the ideas shared by the interviewees. I also explore whether SDO is a relevant correlate of these ideas, testing the predictions outlined above. I also again delineate SDO into its two dimensions and explore how the two dimensions relate to each item. I do this by reworking codes from Chapter 5 into survey items to examine how a larger group of people respond. My first study in this chapter presents a pilot study testing an initial set of items (Study 5A), which is followed by a larger survey testing a refined set of items (Study $5 B)$. 


\section{Study 5A}

\section{Pilot study: Testing quantitative measures of the ideas expressed in the interview study about climate change}

\section{Introduction}

The summary of this study follows the format of a scale creation report. I describe how I developed an initial pool of items based on the codes and themes from Chapter 5. I then present the results of a pilot test of these items that relied on a small convenience sample recruited via social media. The main aim of this study was to develop and refine these items by requesting feedback from participants to improve clarity.

I also report some preliminary data analyses on the items. I included a short measure of SDO to test for possible associations that did not emerge in the exploratory analyses from Study 4. This research is also exploratory, though I do expect to find that SDO is related to many of these ideas, with the predictions and rationales explained above.

I also measured social desirability, which is a useful measure to include in order to see if responses to any items were potentially subject to this bias (e.g. Nederhof, 1985). This is a possibility, as pro-environmental behaviours might be perceived as more positive, though findings on this are mixed (e.g. Félonneau \& Becker, 2008; Milfont, 2009). If any items are strongly associated with this bias, this could suggest that participants are responding to present themselves positively, rather than giving honest opinions, and therefore items may need revising.

\section{Method}

\section{Participants}

Ninety-one participants responded to posts on my own and a colleague's personal Facebook pages requesting participants for a pilot study. As these participants included our own social media contacts, we did not collect any demographic or personal data, so that responses would remain anonymous. Ethical approval was granted by the VUW human ethics committee (Application ID 0000025633). 


\section{Materials}

Measures of social desirability, SDO, and the newly created items were included in a short survey among other items tapping attitudes towards scientific and social issues.

Social desirability. Reynolds' (1982) social desirability scale was used to measure the tendency to respond in socially desirable ways. The scale is comprised of 13 items, including a mix of pro-trait (e.g. "It is sometimes hard for me to go on with my work if I am not encouraged"), and con-trait (e.g. "No matter who I'm talking to, I'm always a good listener") items. Participants are instructed to read the statement and respond by indicating if this is true (coded as 1) or false (coded as 0 ) for them. Because this is a binary measure, I used the Kuder-Richardson formula 20 to assess reliability (statistically equivalent to running Cronbach's alpha in SPSS), which yielded an acceptable scale $(\alpha=.73)$. Once con-trait items were recoded, responses to items were summed together, with higher scores on this scale indicating greater social desirability.

Social dominance orientation. To keep the survey as short as possible, I used an 8item version of Ho et al.'s (2012) $\mathrm{SDO}_{7}$ scale as described in Chapter 3. In this sample, the scale evidenced good reliability both when used as a unidimensional construct $(\alpha=.88)$, and when separated into the dimensions (SDO-D: $\alpha=.87$, SDO-E: $\alpha=.78$ ).

\section{Interview-based environmentalism items.}

I created items by rewording themes and codes from the interview study into survey items. As well as being interested in the extent to which people would agree with the items (i.e. collecting responses using a traditional Likert scale), I wanted to understand how participants would choose between two competing objectives. This is because many interviewees framed action on climate change as an issue of competing interests, for instance requiring them to weigh up environmental concerns with other interests (e.g. in the Not Our Fault, Cost, Motivation, and Time Consuming codes).

To gauge the extent to which participants agreed or disagreed with some ideas, I created the first set of items, to which participants responded using a 7-point Likert scale with response options from strongly disagree to strongly agree. To understand how participants would make a decision when faced with a choice between competing objectives, I created a second set of items where participants were instructed to respond to items on sliding scales, anchored by opposing objectives on each end (e.g. the economy versus the environment), 
with the midpoint labelled 'both equally'. See Figure 6.1 for an example of how the sliding scale items were presented onscreen. The instructions for this set of items was as follows:

"In this next set of questions, we are interested in your opinions on environmental issues.

After reading each statement, we would like you to weigh up the two options on either side of the slider. You can move the slider to the middle, to represent favouring the option that's $50 \%$ of each option on either side, at one of the far sides, or anywhere in between.

We want you to move the slider to where you think best represents your opinion. There are no right or wrong answers here - the best answer is what you think."

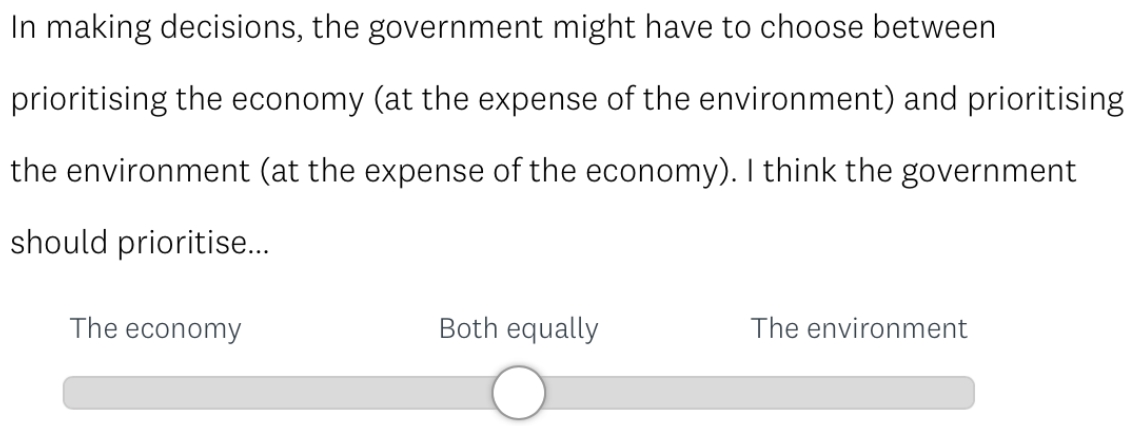

Figure 6.1. Example sliding scale item.

Because of space constraints in the survey, I did not create survey items for all codes. I gave preference to codes where no existing measure captures the same idea. Furthermore, I was still finalising the coding of the interview studies while designing this study, and was therefore unable to create items for codes that were added to the coding scheme after ethical approval was obtained for this study. For more complex ideas, where I was unsure if a single item would be well understood or capture the same construct as the interview-based code, I developed several items for pilot testing. In contrast, for more straight-forward ideas, I developed fewer items.

In the next sections, I detail my process developing items to measure codes belonging to each theme, and my justification for or against including measures of each code in the survey. In a pre-testing phase, I presented an initial list of items to two friends for their comments on any unclear or potentially confusing items. I also discussed the list and made changes with my supervisor. The items below are those that were included in the pilot study. 


\section{Causes and Consequences of Climate Change}

From the Causes and Consequences theme, I created items for the Not Our Fault, Prioritising Profit, and Past Mistakes codes. Despite its popularity amongst interviewees, I did not create items for the Climate Change is Dangerous code as several measures of this construct already exist (e.g. climate change anxiety, perceived threat of climate change). Low Priority and Tourism and Image were excluded due to space constraints and concerns that these codes refer to specific and less commonly cited issues. The Resource Depletion code was added to the coding scheme after the items and data collection for this study were completed.

“Not Our Fault”. This code is consistent with ideas interpreted from other qualitative research (Stoll-Kleemann et al., 2001), though distinct from existing quantitative measures. I developed the following three Likert-style and one sliding scale items (anchors in brackets) to capture the shifting of blame from individuals to industry:

Individuals are not to blame for what polluting industries do, even if we do buy their products.

I feel personally responsible for finding solutions to climate change.

Polluting industries are responsible for finding solutions to climate change.

Some industries generate a lot of pollution, and individuals increase demand by buying their products. Generally, I think that the biggest contributors to climate change are... (Industries - Individuals).

“Prioritising Profit”. This code highlighted the tension between economic and environmental concerns. I created several items with variations on the wording and direction to pilot test:

It is more important for companies to prioritise making profits than minimizing their environmental impact.

It is more important for companies to prioritise minimizing their environmental impact than making profits.

In making decisions, companies might need to choose between prioritizing making profits, and prioritizing minimizing their environmental impact. I think that companies should prioritize... (Making profits - Minimizing environmental impact). 
Although the code captured mentions of this tension for businesses, I also included similar measures for the role of government:

The government should prioritise the economy, even if this is at the expense of the environment.

The government should prioritise the environment, even if this is at the expense of the economy.

In making decisions, the government might have to choose between prioritising the economy (at the expense of the environment) and prioritising the environment (at the expense of the economy). I think the government should prioritise... (The economy The environment).

To capture this idea without tying it to government or business directly, I also included three general items to measure the tension between economic and environmental concerns:

Economic growth is bad for the environment.

Some people think that acting on climate change will have negative effects on the economy. Others think it could have positive effects. I think that short-term, acting on climate change will have... (Negative effects of the economy - Positive effects on the economy).

Some people think that acting on climate change will have more negative effects than positive on the economy. Others think it could have positive effects because it will avoid potentially costly damage from climate change. I think that long-term, acting on climate change will have... (Negative effects on the economy - Positive effects on the economy).

“Past Mistakes”. I created one item to measure this code quantitatively:

Climate change has mostly been caused by people in the past making mistakes.

\section{Hopeless Environmentalist}

From the Hopeless Environmentalist theme, I developed survey items for many of the personal barriers to action. I excluded those that were developed in the later stages of coding (Forgetful or Don't Think, Emotion, Self as Hypocritical), and those with already established 
measures (Psychological Distance codes). I also excluded the Not in a Position to Help code, as I thought this was an issue specific to first year student samples living in halls of residence, likely unrelated to ideology (see Chapter 5 Discussion).

"Cost". To measure the perception that action on climate change is costly, I created the following two items:

I'm happy to spend more on a product if it's environmentally friendly.

Acting on climate change is going to cost more than not acting.

“Motivation". To measure the tension between convenience and effort that interview participants mentioned, I created the following items:

I won't do an environmentally friendly action if it's going to take a lot more effort.

Some behaviours are less convenient than others, but are more environmentally

friendly (e.g. walking instead of driving). In my daily life, I tend to choose behaviours that are... (100\% of the time environmentally unfriendly, but convenient $-100 \%$ of the time environmentally friendly, but less convenient).

"Self-efficacy". This code overlapped with the Not Our Fault code, though I added one additional item:

No matter how many little things that individuals do to try and stop climate change, it won't make a difference.

“Time Consuming". I included one item to measure this code:

Acting environmentally friendly is too time consuming.

"Stigma". This was a less common code, though unique in the literature and therefore I still included one item in the survey:

I would be embarrassed if my friends thought I was becoming an 'environmentalist'.

"Climate Change is Natural". Recall that participants in the interview study generally did not deny that climate change is happening, but instead expressed confusion or scepticism about its anthropogenic causes. Many scales exist to measure belief in anthropogenic climate change (e.g. single item "climate change is caused by humans") and the certainty around this (e.g. scientific consensus). However, they do not capture the degree to which individuals think it is human activity versus natural causes that are responsible for 
recent changes in the climate. I created one item to attempt to capture this by putting the two causes as competing objectives on a sliding scale:

In my opinion, climate change is caused by... (100\% human activity - 100\% natural causes).

\section{Solutions}

From the Solutions theme, I included items to measure several of the codes from both the Top-Down and Bottom-Up subthemes. I did not develop items for Awareness and Education, Infrastructure, Leading by Example, Global Agreements, and Food Choices codes, as they were developed more recently.

I included some general items to measure the belief that solutions should come from the top:

The government is responsible for finding solutions to climate change.

I believe that the responsibility to find solutions to climate change is down to... (The government alone - Individuals alone).

I also included an item measuring the extent that participants believed they ought to come from the top versus the bottom (individual action):

Some solutions to climate change can come from the government (e.g. policy decisions, law changes), and others can come from the people (individuals making personal changes to their lifestyle). I think that environmental action should come from... (The government alone - Individuals alone).

“Regulations”. I included several items tapping support for regulations to be introduced to combat climate change:

I would rather change my behaviour for the environment because I want to, not because a law tells me to.

The government should introduce fines to reduce environmentally unfriendly practices.

Businesses shouldn't have to worry about their environmental impact: The government should stay out of it. 
It might be necessary to raise funds to go towards solutions to climate change by creating new taxes. These could come from taxing environmentally unfriendly industries (coming out of their profits), or be added to the cost of the product (for individuals to pay for). I would prefer for the new taxes to be applied to... (Environmentally unfriendly industries - The cost of environmentally unfriendly products).

“New Technology Will Save Us". This code appeared to capture a novel idea. To measure this quantitatively, I created two items:

We don't need to worry about climate change, because new technologies will eventually solve our environmental problems.

Economic growth tends to be accompanied by an increase in greenhouse gas emissions. However, some people think that economic growth is good for the environment, because new technologies will be developed to fix the problem of climate change. In my opinion, economic growth is... (Harmful for the environment Helpful for the environment).

“Incentives". I created two items for the 'Incentives' code:

The government should introduce incentives to promote environmentally friendly practices.

The government might want to introduce fines to reduce environmentally unfriendly practices, or incentives to promote environmentally friendly practices. I think that the government should focus on... (Introducing fines - Introducing incentives).

“Little Things”. The 'Little Things' code was common among the interviews. Although it captures the idea that people should take many small actions on climate change, which scales already exist to measure (e.g. pro-environmental behaviours measures), to my knowledge none capture the unique, often verbatim aspect of 'just doing little things'. I was unsure the best way to translate this idea into quantitative survey items, so I created several options and wording variations for pilot testing:

It's enough for everyone to do little things to prevent climate change, like recycling and turning lights off. 
I would rather make a few big changes for the environment (e.g. going zero waste or vegan) than making lots of little changes (e.g. recycling and turning off lights).

I would rather make lots of little changes for the environment (e.g. recycling and turning off lights) than a few big changes (e.g. going zero waste or vegan).

One way for an individual to act on climate change is to do lots of little things, such as recycling or turning off lights. Another way is to make a few big lifestyle changes, like installing solar panels or going zero waste. I would prefer to act by... (100\% of efforts to come from doing lots of little things $-100 \%$ of efforts to come from a few big things).

The final item to measure this idea drew both on the 'little things' code and the 'collective action' code:

One way to act on climate change is for a majority of people to do little things, such as recycling or turning off lights. Another way is to get a minority of people to make big changes to their lifestyles, like installing solar panels or going zero waste. I believe that the best way for individuals to act on climate change is for efforts to come from... (A majority doing little things - A minority doing big things).

“Collective Action". As well as the item above, I developed the item below to capture the 'tragedy-of-the-commons' idea (also related to Self-Efficacy):

I don't want to have to make changes to my lifestyle to help the environment unless everyone else does too.

"Co-benefits". The following item was written to measure support for environmentally friendly actions that come with other benefits:

I will do environmentally friendly behaviours if I get something out of it, like a cheaper power bill by conserving energy.

“Public pressure". To measure this code, I created two items:

The best way for individuals to help the environment is to put pressure on the government to make changes from above.

Individuals can act on climate change by doing small things to reduce their environmental impact (e.g. recycling), or by putting pressure on the government and 
big corporations to make changes. I would prefer to act by... (100\% of efforts to come from doing small things $-100 \%$ of efforts to come from putting pressure on the government and corporations).

"Habit". The following items were created based on an earlier iteration of the Habit code, where I noted that some extracts talked about making lifestyle changes for the environment, and others talked about being resistant to change (a code that was later deleted as it had too few extracts to form a coherent definition):

We should try to change our lifestyles in order to reduce our impact on the environment.

I don't want to make big changes to the way I live my life, even if it would be better for the environment.

Individuals shouldn't be expected to make changes to their lifestyles for the environment.

Deep sea drilling. I also created one item based on the discussions of deep sea drilling (not included in my thesis). Many participants suggested profits from deep sea drilling ought to go to the community, and others suggested they go to corporations. I developed the following item which drew on a hypothetical scenario to be consistent with Jackson et al.'s (2013) and Milfont et al.'s (2014) vignettes:

Imagine some minerals are found on public land, and a private company invests in setting up a mining operation. You are asked to allocate the profits from the mine, either to the investors, to the community, or divided between them in some way. I think that resources should go to... (The investors - The community).

\section{Procedure}

A colleague and I advertised the link to the survey on our personal Facebook pages, and posted it on a public Facebook group that features discussions about New Zealand politics. Participation in the survey was voluntary, took approximately 20 minutes, and participants were told they could withdraw at any time.

I spaced the new items out on separate pages, with 15 Likert scale items or 5 slider items per page. To gather feedback on these new items, I presented participants with the following instructions at the end of each page: "This set of items are under development, and 
we would like your feedback on how to improve them. Can you please tell us if any of these items were confusing, unclear, or could be improved in any way?" Responses were collected using open text boxes.

On completion of the survey, participants read an onscreen debrief, and were presented with a link to a separate Google Docs sheet to record their email address if they wished to enter the draw to win a double movie pass. This was not linked to their survey responses to ensure there was no way to identify individual participant's responses in the dataset.

\section{Analytic approach}

My approach to the data was to first examine the open-ended responses requesting feedback on the items, in order to note any issues that may affect interpretation of the data (e.g. misunderstood items). I then analysed univariate statistics for each set of items, including means, one-sample t-tests to examine how the mean diverges from the midpoint of each scale, and standard deviations. Lastly, I conducted bivariate analyses to see whether and how the items relate to social desirability bias and SDO. Responses to Likert and sliding scale items were analysed and are presented separately.

\section{Results}

\section{Participant's feedback on piloted items}

The majority of participants left the feedback boxes blank, though I received between five and ten comments on each question block. Many comments were not on the survey items at all (e.g. "I need a coffee!"), and so were disregarded. Others were positive, and did not suggest changes (e.g. "all quite clear", "they were great", "perfect").

Of the comments that did suggest changes, almost all claimed the questions were repetitive (e.g. "Remove repetitive questions", "One of the questions... was duplicated", "So repetitive"). I did ask several similar questions (e.g. impacts on the economy short and long term), and although I put these in different blocks, participants may not have noticed subtle differences in wording. As the aim of the pilot was to test which of the questions to include in a larger survey, I disregarded these comments, as the final survey would be shortened to choose the best of similarly worded items anyway. However, understanding participant's experiences of completing many similar items is a useful reminder to researchers who use multi-item scales for greater reliability. It is especially important to consider the impact of 
this on responses when participants do not detect slight differences in wording, and also how longer scales may contribute to fatigue effects.

A few participants did not like that they had to choose between competing objectives in the sliding scale questions (e.g. "Need a neither option"). I employed this response type as I wanted participants to weigh up each option and decide where their opinion fit between them. Having participants choose between values is effective in encouraging them to make decision between them, as when given the opportunity to instead rate values tends to result in each getting equal, high ratings of importance, and hence avoiding deciding between them (Krosnick \& Alwin, 1988). Because none of the comments suggested changing or removing any of the questions in particular to improve the quality or clarity of the survey, I did not believe that any changes to the items were necessary based on this feedback, and therefore moved on to the statistical analysis.

\section{Planned analyses of Likert-scale responses}

Descriptive statistics. Means and standard deviations for each item are reported in Table 6.1. One sample t-tests revealed that while the means for most items were close to the midpoint of the scale ( 4 on a 7-point scale), in all but two cases (items $15 \& 18$ ) the means were significantly different from the midpoint, and all indicated that responses are on average slightly skewed towards pro-environmentalism. Standard deviations were relatively small, indicating that while responses showed some variability, scores tended to cluster around the means. 
Table 6.1.

Descriptive statistics for the Likert response items, and their associations with SDO.

Correlations with SDO and its dimensions

Environmental attitude item
1. I'm happy to spend more on a product if it's environmentally
friendly

2. I won't do an environmentally friendly action if it's going to take a lot more effort $(\mathrm{R})$

3. Acting on climate change is going to cost more than not acting $(\mathrm{R})$

4. It's enough for everyone to do little things to prevent climate change, like recycling and turning lights off (X)

5. I don't want to make big changes to the way I live my life, even if it would be better for the environment $(\mathrm{R})$

6. We should try to change our lifestyles in order to reduce our impact on the environment

7. Individuals are not to blame for what polluting industries do, even if we do buy their products (R)

8. I would rather make a few big changes for the environment (e.g. going zero waste or vegan) than making lots of little changes (e.g. recycling and turning off lights) (X)

9. No matter how many little things that individuals do to try and stop climate change, it won't make a difference (R)

Code

$M$

(SD)

Social

desirability

5.25

(1.55)

$-.157$

3.00

Motivation

3.00
$(1.70)$

Cost

2.77

Little

Things

3.00

(1.80)

Little $\quad 2.94$

Things

(1.77)

Habit

$$
5.80
$$

Not Our

3.08

Fault

(1.71)

Little

3.31

Things

(1.47)

$$
-.274 *
$$

.199

.034

$-.149$

.162

.123 
10. I would rather make lots of little changes for the environment (e.g. recycling and turning off lights) than a few big changes (e.g. going zero waste or vegan) $(\mathrm{X})$

11. The best way for individuals to help the environment is to put pressure on the government to make changes from above (X)

12. I will do environmentally friendly behaviours if I get something out of it, like a cheaper power bill by conserving energy $(\mathrm{X})$

13. I would rather change my behaviour for the environment because I want to, not because a law tells me to $(\mathrm{X})$

14. I don't want to have to make changes to my lifestyle to help the environment unless everyone else does too (R)

15. I feel personally responsible for finding solutions to climate change

16. Acting environmentally friendly is too time consuming $(\mathrm{R})$

17. I would be embarrassed if my friends thought I was becoming an 'environmentalist' (R)

18. Climate change has mostly been caused by people in the past making mistakes (X)

19. The government should prioritise the economy, even if this is at the expense of the environment $(\mathrm{R})$

20. The government should prioritise the environment, even if this is at the expense of the economy

21. Individuals shouldn't be expected to make changes to their lifestyles for the environment (R)

\begin{tabular}{|c|c|c|c|c|c|}
\hline $\begin{array}{l}\text { Little } \\
\text { Things }\end{array}$ & $\begin{array}{c}4.57 \\
(1.48)\end{array}$ & -.111 & -.005 & .004 & -.013 \\
\hline $\begin{array}{l}\text { Public } \\
\text { Pressure }\end{array}$ & $\begin{array}{c}5.18 \\
(1.67)\end{array}$ & -.090 & $-.289 *$ & $-.254^{*}$ & $-.285^{*}$ \\
\hline Co-benefits & $\begin{array}{c}4.96 \\
(1.81)\end{array}$ & -.151 & .063 & .038 & .081 \\
\hline Regulations & $\begin{array}{c}4.52 \\
(1.68)\end{array}$ & -.032 & -.003 & -.048 & .047 \\
\hline $\begin{array}{c}\text { Collective } \\
\text { Action }\end{array}$ & $\begin{array}{c}2.56 \\
(1.48)\end{array}$ & -.081 & .065 & .054 & .068 \\
\hline $\begin{array}{c}\text { Not Our } \\
\text { Fault }\end{array}$ & $\begin{array}{c}3.70 \\
(1.75)\end{array}$ & .059 & $-.248 *$ & -.184 & $-.282 *$ \\
\hline $\begin{array}{c}\text { Time } \\
\text { Consuming }\end{array}$ & $\begin{array}{c}2.56 \\
(1.36)\end{array}$ & -.092 & $.254^{*}$ & $.259 *$ & .208 \\
\hline Stigma & $\begin{array}{c}2.04 \\
(1.54)\end{array}$ & -.046 & $.314 * *$ & $.284 *$ & $.300 *$ \\
\hline $\begin{array}{c}\text { Past } \\
\text { Mistakes }\end{array}$ & $\begin{array}{c}4.27 \\
(1.59)\end{array}$ & -.109 & .074 & .063 & .076 \\
\hline $\begin{array}{l}\text { Prioritising } \\
\text { Profit }\end{array}$ & $\begin{array}{c}2.26 \\
(1.63)\end{array}$ & .111 & $.419 * * *$ & $.374 * *$ & $.405^{* *}$ \\
\hline $\begin{array}{l}\text { Prioritising } \\
\text { Profit }\end{array}$ & $\begin{array}{c}5.16 \\
(1.74)\end{array}$ & .162 & $-.387 * *$ & $-.314 * *$ & $-.409 * * *$ \\
\hline Habit & $\begin{array}{c}2.16 \\
(1.39)\end{array}$ & .028 & $.309 * *$ & .192 & $.392 * *$ \\
\hline
\end{tabular}


22. The government is responsible for finding solutions to climate change (R)

23. The government should introduce fines to reduce environmentally unfriendly practices

24. The government should introduce incentives to promote environmentally friendly practices

25. Polluting industries are responsible for finding solutions to climate change (R)

26. Economic growth is bad for the environment $(\mathrm{X})$

27. We don't need to worry about climate change, because new technologies will eventually solve our environmental problems $(\mathrm{R})$

28. It is more important for companies to prioritise making profits than minimizing their environmental impact $(\mathrm{R})$

29. It is more important for companies to prioritise minimizing their environmental impact than making profits

30. Businesses shouldn't have to worry about their environmental impact: The government should stay out of it (R)

\begin{tabular}{|c|c|c|c|c|c|}
\hline Top-Down & $\begin{array}{c}5.44 \\
(1.60)\end{array}$ & -.149 & $-.295^{*}$ & -.177 & $-.381 * *$ \\
\hline Regulations & $\begin{array}{c}5.85 \\
(1.56)\end{array}$ & .106 & $-.386^{* *}$ & $-.278^{*}$ & $-.447 * * *$ \\
\hline Incentives & $\begin{array}{c}6.33 \\
(1.09)\end{array}$ & -.045 & -.225 & $-.240^{*}$ & -.174 \\
\hline $\begin{array}{l}\text { Not Our } \\
\text { Fault }\end{array}$ & $\begin{array}{c}5.42 \\
(1.49)\end{array}$ & -.150 & -.182 & -.092 & $-.252 *$ \\
\hline $\begin{array}{l}\text { Prioritising } \\
\text { Profit }\end{array}$ & $\begin{array}{c}2.93 \\
(1.59)\end{array}$ & .131 & -.203 & -.164 & -.215 \\
\hline New Tech & $\begin{array}{c}2.12 \\
(1.21)\end{array}$ & .012 & $.357 * *$ & .206 & $.470 * * *$ \\
\hline $\begin{array}{l}\text { Prioritising } \\
\text { Profit }\end{array}$ & $\begin{array}{c}2.33 \\
(1.59)\end{array}$ & .028 & $.485^{* * *}$ & $.394 * *$ & $.512 * * *$ \\
\hline $\begin{array}{l}\text { Prioritising } \\
\text { Profit }\end{array}$ & $\begin{array}{c}4.99 \\
(1.66)\end{array}$ & .134 & $-.465 * * *$ & $-.357 * *$ & $-.513 * * *$ \\
\hline Regulations & $\begin{array}{c}1.62 \\
(1.34)\end{array}$ & .108 & $.381 * *$ & .225 & $.496^{* * *}$ \\
\hline
\end{tabular}

Note. ${ }^{* * *} p<.001, * * p<.01,{ }^{*} p<.05$. $\mathrm{R}$ indicates that the item was reverse coded during exploratory analyses, $\mathrm{X}$ indicates items removed during exploratory analyses. 
Associations with social desirability. The analyses reported in Table 6.1 reveal that only one item (Item 2: I won't do an environmentally friendly action if it's going to take a lot more effort) was significantly related to social desirability $(r=-.27)$. This association was weak and negative, and is interpreted as meaning that greater endorsement of this item relates to lower social desirability scores. This makes sense, as individuals who admit to not engaging in effortful pro-environmental actions may care less about being regarded positively. Scores for the remaining 29 items were unrelated to social desirability. Social desirability bias is therefore unlikely to affect responding to the majority of these items.

Associations with SDO. In Table 6.1, I also correlated each item with SDO scores, and SDO-D, and SDO-E subscale scores. Eighteen items significantly related to at least one aspect of SDO. In all cases, higher scores on SDO related to disagreement with proenvironmental item content (e.g. negative relationship with item 15: I feel personally responsible for finding solutions to climate change), and endorsement of anti-environmental items (e.g. positive relationship with item 17: I would be embarrassed if my friends thought I was becoming an 'environmentalist'). This is consistent with the general trend where higher SDO is associated with lower pro-environmentalism (Chapter 2).

Additionally, my predictions were also generally supported, with SDO negatively associated with items that emphasize prioritising the environment over the economy or profits (items 21, 26, 29), instead positively relating to items which emphasize prioritising profit (items 19, 28). Unexpectedly, several of the items designed to measure Bottom-Up codes were not related to SDO. These were the items designed to measure support for Little Things (item 4, 5, 8, 9, 10) and Collective Action (item 14). However, support for Public Pressure (item 11) and Habit (item 21) were related. Opposing Regulations was unrelated to SDO in item 13, but was related in items 23 and 30, suggesting that the wording and focus (e.g. on introducing fines, versus government staying out of business affairs) may impact whether responses are ideologically motivated. The Incentives item (24) was unrelated to SDO overall, but was related to SDO-D.

The Motivation item (2) was related to SDO overall, indicating that the greater effort associated with pro-environmental behaviours is a barrier for them. Other personal barriers were also endorsed more often with higher scores on the SDO scale, e.g. Time Consuming (item 16), Stigma (17), and Cost (item 1 significant, item 3 unrelated). Supporting 
predictions, item 27, which was based on the New Technology Will Save Us code, was related to greater endorsement of SDO.

Consistent with the findings reported in Chapter 3, in all but two cases SDO-E was more strongly associated with the environmental items than SDO-D. Notable exceptions to this were items 16 (acting environmentally friendly is too time consuming) and 24 (The government should introduce incentives to promote environmentally friendly practices), where SDO-D was more strongly associated.

\section{Exploratory analyses of Likert-scale responses}

My intentions for the items created in this study was to examine separately their associations with SDO. However, if the items work together to form a scale, it would be the first scale to my knowledge created using a data-driven approach using lay people's own ideas on the environment. This is the case both for general environmental research, and for research on SDO and the environment. Here, I outline the steps I took towards exploring this possibility.

Step 1. First, I reverse coded relevant items so that all were scored in the proenvironmental direction. For nine items, it was unclear whether it was a 'pro' or 'anti' environmental response (items 4, 8, 10, 11, 13, 18, 22, 25, 26, marked with an X in Table 6.1). However, this is a somewhat subjective decision. For example, individuals who are generally pro-environmental might be more likely to agree to item 4 ("It's enough for everyone to do little things to prevent climate change, like recycling and turning lights off'), as they support individual action on climate change. However, they might also be more likely to disagree, because they think individual action is not enough and support additional action on top of this.

I examined the correlations between items carefully, and indeed these items in question had very low associations with the rest of the items (i.e. $r$ 's between $+/-.2$ ), suggesting that even if those with negative correlations were reverse-coded, they do not belong in a scale, and thus supporting their removal from these analyses. Items 22 and 25 instead had relatively stronger negative associations, justifying their reverse-coding and inclusion in the next step. This was surprising as these items, which argue that government and business are responsible for finding solutions to climate change, may appear at face value to be pro-environmental. The interpretation of them as anti-environmental, and justified by the statistical analysis, is that the items are shifting responsibility (Not Our Fault). 
Step 2. I then conducted scale reliability analyses in SPSS of the remaining 22 items, which yielded an acceptable Cronbach's alpha $(\alpha=.90)$. Examination of the corrected itemtotal correlations revealed all were within an acceptable range (.27 to .73).

Step 3. I created a mean score for each participant on the 22-item scale by calculating a mean for the 22 items $(1,2,3,5,6,7,9,14,15,16,17,19,20,21,22,23,24,25,27,28$, $29,30)$. Higher scores on this scale indicated greater pro-environmentalism. Mean scores ranged from 2.59 to 6.91 , with a relatively high mean of $5.46(\mathrm{SD}=.90)$, above the midpoint on the 7-point scale. This indicated that my sample tended towards pro-environmentalism, and that the majority of participants scored close to this mean.

Step 4. The previous steps showed that the scale demonstrated good internal reliability, with the items fitting together well and potentially representing a general factor. The scale should demonstrate convergent validity by correlating positively with climate change belief (measured using two items: Climate change is real, and Climate change is caused by humans, $r=.59$ ), and negatively with SDO. I expected the scale to demonstrate discriminant validity with a null association with social desirability.

Consistent with these expectations, I found that the new measure correlated positively with climate change belief $(r(66)=.55, p<.001)$, indicating that higher scores on the new measure were associated with greater belief in climate change. The new measure was also negatively related to SDO $(r(67)=-.58, p<.001$; and both the anti-egalitarian dimension: $r(67)=-.59, p<.001$, and dominance dimension: $r(67)=-.48, p<.001)$. Providing evidence of discriminant validity, the new measure was unrelated to social desirability $(r(71)=.00, p=$ .997).

\section{Planned analyses of sliding scale responses}

Descriptive statistics. Participants responded to the next set of items on sliding scales. Scores ranged from 0 (representing complete endorsement with the objective on the left) to 100 (complete endorsement with right-hand side objective), with a midpoint of 50 signifying equal endorsement of each objective. A mean below 50, therefore, means that on average the sample tend to prefer the target objective to the left of the scale, and a mean above 50 is interpreted as meaning that participants tend to prefer the objective to the right. In line with results based on Likert responses, Table 6.2 reveals that on items where a proenvironmental direction could be determined, participants tended to choose closer to the proenvironmental option. The favoured objective is bolded in the table. The means were 
significantly different from the midpoints for all but item 9, and standard deviations were moderate (about 20 on a 100-point scale), indicating a good level of variability on these items.

Associations with social desirability. Next, I correlated each item with social desirability. Three items significantly correlated: Item 3, 14, and 15. These associations indicate that: 1. Greater support for individuals (as opposed to government) as responsible for environmental action is associated with lower scores in social desirability (thus unlikely to be an issue); 2. Agreeing that people should put pressure on the government and corporations (rather than individuals) to act is associated with higher social desirability; and 3. The belief that acting on climate change will have long term positive (rather than negative) effects on the environment is associated with higher social desirability. All other items were unrelated to social desirability. In all cases, controlling for social desirability did not change the direction or significance of the association between these items and SDO. Specifically, item 12 remained significantly positively related to SDO after controlling for social desirability bias $(\beta=.31, p=.028)$, item 14 remained unrelated to $\operatorname{SDO}(\beta=-.16, p=.247)$, and SDO remained a strong negative predictor of item $15(\beta=-.50, p<.001)$. 
Table 6.2.

Descriptive statistics of sliding scale responses and their associations with social desirability and SDO.

Mean

(SD) Soc. Des SDO SDO-D SDO-E

1. In making decisions, the government might have to choose between prioritising the economy (at the expense of the environment) and prioritising the environment (at the expense of the economy). I think the government should prioritise... (The economy -

67.15 The environment)

2. One way for an individual to act on climate change is to do lots of little things, such as recycling or turning off lights. Another way is to make a few big lifestyle changes, like installing solar panels or going zero waste. I would prefer to act by... (100\% of efforts to come from doing lots of little things - $100 \%$ of efforts to come from a few big things)

3. Some solutions to climate change can come from the government (e.g. policy decisions, law changes), and others can come from the people (individuals making personal changes to their lifestyle). I think that environmental action should come from... (The government alone - Individuals alone)

4. Imagine some minerals are found on public land, and a private company invests in setting up a mining operation. You are asked to allocate the profits from the mine, either to the investors, to the community, or divided between them in some way. I think that resources should go to... (The investors - The community)

5. Some industries generate a lot of pollution, and individuals increase demand by buying their products. Generally, I think that the biggest contributors to climate change are... (Industries - Individuals)

6. Economic growth tends to be accompanied by an increase in greenhouse gas emissions. However, some people think that economic growth is good for the environment, because new technologies will be developed to fix the problem of climate change. In my opinion, economic growth is... (Harmful for the environment - Helpful for the environment) $\begin{array}{lll}-.431 * * * & -.361 * * & -.440 * * *\end{array}$

42.85

(17.80)

$-.013 \quad .067 \quad .003$

.132

41.98

$-.303^{*}$

$-.108$

$.378 * *$

$.344 * *$

$.363 * *$

44.34

(19.95) 
7. Some behaviours are less convenient than others, but are more environmentally friendly (e.g. walking instead of driving). In my daily life, I tend to choose behaviours that are... (100\% of the time environmentally unfriendly, but convenient $-100 \%$ of the time environmentally friendly, but less convenient)

8. In making decisions, companies might need to choose between prioritizing making profits, and prioritizing minimizing their environmental impact. I think that companies should prioritize... (Making profits - Minimizing environmental impact)

9. Some people think that acting on climate change will have negative effects on the economy. Others think it could have positive effects. I think that short-term, acting on climate change will have... (Negative effects of the economy - Positive effects on the economy)

10. The government might want to introduce fines to reduce environmentally unfriendly practices, or incentives to promote environmentally friendly practices. I think that the government should focus on... (Introducing fines - Introducing incentives)

11. It might be necessary to raise funds to go towards solutions to climate change by creating new taxes. These could come from taxing environmentally unfriendly industries (coming out of their profits), or be added to the cost of the product (for individuals to pay for). I would prefer for the new taxes to be applied to... (Environmentally unfriendly industries - The cost of environmentally unfriendly products)

12. I believe that the responsibility to find solutions to climate change is down to... (The government alone - Individuals alone)

13. One way to act on climate change is for a majority of people to do little things, such as recycling or turning off lights. Another way is to get a minority of people to make big changes to their lifestyles, like installing solar panels or going zero waste. I believe that the best way for individuals to act on climate change is for efforts to come from... (A majority doing little things - A minority doing big things) 
14. Individuals can act on climate change by doing small things to reduce their environmental impact (e.g. recycling), or by putting pressure on the government and big corporations to make changes. I would prefer to act by... (100\% of efforts to come from doing small things $\mathbf{- 1 0 0 \%}$ of efforts to come from putting pressure on

58.18 the government and corporations)

15. Some people think that acting on climate change will have more negative effects than positive on the economy. Others think it could have positive effects because it will avoid potentially costly damage from climate change. I think that long-term, acting on climate change will have... (Negative effects on the economy - Positive effects on the economy)

16. In my opinion, climate change is caused by... (100\% human activity $-100 \%$ natural causes)

Note. ${ }^{* *} p<.001, * * p<.01,{ }^{*} p<.05$. Soc. Des - Social Desirability

To interpret the mean: The scale ranges from 0 to 100, with the objective on the left coded as 0 at the most extreme end (thus favouring this objective means scoring between 0 and 50), and on the right coded as $100(50-100)$. A mean of 50 means that on average, people gave equal weighting to either objective. Where the mean indicates that one objective tends to be favoured over another, this objective is in bold. 
Associations with SDO. Next, I correlated each item with SDO (total) and its two dimensions. Table 6.2 shows that 12 of the 16 items were significantly correlated with SDO. To interpret these, positive correlations indicate that endorsement of SDO is related to scores closer to the objective on the right side of the sliding scale (relative to lower endorsement of SDO), and negative correlations mean that higher SDO relates to relatively greater endorsement of left side objectives. These associations suggest that the higher someone scores on SDO, the more they agree (relative to those low on SDO) that:

- Governments and corporations should prioritise the economy over the environment,

- Resources from a mine should benefit investors over the community,

- Individuals contribute more to pollution than industries,

- Economic growth is helpful, rather than harmful, to the environment,

- Both short and long term, acting on climate change will more negatively affect the environment,

- Climate change is caused by natural causes over human activity, and

- They tend to choose less environmentally friendly behaviours.

This is not to say that high-SDOs endorse the objectives above, but they endorse it to $a$ greater extent than low-SDOs. This is illustrated further by the next set of analyses.

\section{Exploratory analyses of sliding scale responses}

To better illustrate how high- and low-SDOs place their opinions on the sliding scales, I used the two groups created previously (high-SDO and low-SDO) and compared groups' mean responses to each item using independent sample t-tests. The results are illustrated in Figure 6.2 below.

For nine of the 16 items, there is a significant difference between groups, with highSDO's tending to choose the anti-environmental option more than low-SDOs, who tend to place their preference relatively closer to the pro-environmental objective. They also show that it is not the case that high-SDOs think climate change is unrelated to human activity. Instead, the figure shows that both groups place their opinion on the side that recognises that human activity has some effect on climate change. However, individuals lower in SDO tend to place blame with human activity to a greater extent than high-SDOs. In three cases, this 
pattern does flip so that the groups on average place themselves on different sides of the scale. For instance, high-SDOs perceive a negative short-term economic impact of acting on climate change, whilst low-SDOs perceive a positive impact.

Taken together, these differences are impressive, considering the sample sizes are small ( $n$ s of 30 and 43), and groups were separated based on a mean-split, with the high-SDO group still scoring below the midpoint on the SDO scale. 
$100 \%$ human

activity

$100 \%$ of efforts to come from doing lots of little things

$100 \%$ of efforts to come from doing small things

A majority doing little things

Environmentally

unfriendly

industries

Introducing fines

Negative effects on the economy

Negative effects

on the economy
$*[$

In my opinion, climate change is caused by ...

One way for an individual to act on climate change is to do lots of little things, such as recycling or turning off lights. Another way is to make a few big lifestyle changes, like installing solar panels or going zero waste. I would prefer to act by...

$$
\text { ns }[
$$

Individuals can act on climate change by doing small things to reduce their environmental impact (e.g. recycling). or by putting pressure on the government and big corporations to make changes. I would prefer to act by ...

\section{] ns}

One way to act on climate change is for a majority of people to do little things, such as recycling or turning off lights. Another way is to get a minority of people to make big changes to their lifestyles, like installing solar panels or going zero waste. I believe that the best way for individuals to act on climate change is for efforts to come from.

$$
\text { ns }[
$$

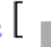$$
\text { [ }
$$

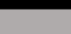

It might be necessary to raise funds to go towards solutions to climate change by creating new taxes. These could come from taxing environmentally unfriendly industries (coming out of their profits), or be added to the cost of the product (for individuals to pay for). I would prefer for the new taxes to be applied to...

The government might want to introduce fines to reduce environmentally unfriendly practices, or incentives to promote environmentally friendly practices. I think that the government should focus on.

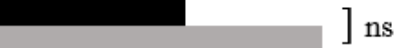

\section{ns}

Some people think that acting on climate change will have negative effects on the economy. Others think it could have positive effects. I think that short-term, acting on climate change will have.

$*[$

Some people think that acting on climate change will have more negative effects than positive on the economy. Others think it could have positive effects because it will avoid potentially costly damage from climate change. I think that long-term, acting on climate change will have

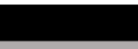

$-30$

$-20$
$-10$
$100 \%$ natural

causes

$100 \%$ of efforts to come from a few big things

$100 \%$ of efforts to come from putting pressure on government an corporations

A minority doing big things

The cost of environmentally unfriendly products

Introducing incentives

Positive effects on the economy

Positive effects on the economy

- High 1 Low 
In making decisions, companies might need to choose between prioritizing making profits, and prioritizing minimizing their environmental impact. I think that companies should prioritize.

Making profits

$100 \%$ of the time environmentally

unfriendly, but

convenient

Harmful for the

environment

ndustries

The government

alone

The government

lone

The economy
Some behaviours are less convenient than others, but are more environmentally friendly

(e.g. walking instead of driving). In my daily life, I tend to choose behaviours that are..

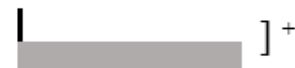

Economic growth tends to be accompanied by an increase in greenhouse gas emissions. However, some people think that economic growth is good for the environment, because new technologies will be developed to fix the problem of climate change. In my opinion, economic growth is...

Some industries generate a lot of pollution, and individuals increase demand by buying

their products. Generally, I think that the biggest contributors to climate change are.

$+[$

Imagine some minerals are found on public land, and a private company invests in setting up a mining operation. You are asked to allocate the profits from the mine, either to the investors, to the community, or divided between them in some way. I think that the resources should go to...

I believe that the responsibility to find solutions to climate change is down to...

$$
*[
$$

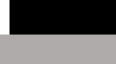

Some solutions to climate change can come from the government (e g policy decisions, law changes)

and others can come from the people (individuals making personal changes to their lifestyle). I think that environmental action should come from.

$$
\text { makin }
$$

In making decisions, the government might have to choose between prioritising the economy (at the

expense of the environment) and prioritising the environment (at the expense of the economy). I think the government should prioritise...

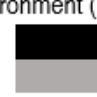

Minimizing environmental impact $100 \%$ of the time environmentally friendly, but less convenient

\section{Helpful for the} environment

Individuals

The community

Individuals alone

Individuals alone

The environment

Figure 6.2. Mean differences between high- and low-SDO groups on the sliding scale items. 


\section{Comments}

The analyses reported in Study 5A indicate that people tend towards agreeing with the pro-environmental ideas reported in the interviews. This is consistent with scores on other environment-relevant variables (e.g. higher than the midpoint means on NEP, climate change belief, see Chapter 3). Furthermore, many of the newly created items are significantly associated with SDO (and for most, I did not find evidence that these are related to social desirability). The Likert scale items also show promise for forming a scale that correlates strongly with endorsement of SDO. Similarly, when separated into groups of high- and lowSDO, participants responses to the items were significantly different. Importantly, these differences are also broadly consistent with the predictions based on previous literature.

However, because this was a pilot study, there are limitations to the results presented here. For instance, the sample size was small relative to the number of items, and unlikely to be representative of the target population, given that the sampling frame was friends and family of myself and a colleague (i.e. a convenience sample). Related to this, we decided not to collect demographic data to protect the privacy of our participants, precluding examination of the representativeness of the sample. The content of the survey was also limited in scope, with only the new items, and several other basic, short scales included. Regardless, the items do show promise for identifying SDO-specific environmental attitudes, and I therefore build on this work in Study 5B. 


\section{Study 5B}

\section{How does social dominance orientation relate to student's ideas about climate change?}

\section{Introduction}

I expand on the testing of items piloted in the previous Study (5A), which were in turn based on ideas shared by the interviewees in Study 4. For consistency throughout this line of work, I recruited a larger sample from the same population (first year students). I also took several additional steps for a more thorough examination of these constructs and their associations.

In the meta-analyses and longitudinal research presented in Chapters 2 and 3, I included RWA as a second ideological correlate of environmentalism. These studies demonstrated that both together (using correlation) and independently (using regression analyses), RWA and SDO are associated with a range of environment-relevant variables and attitudes on climate change. Due to space restrictions, I did not include a measure of RWA in Study 5A. However, I include it in this study to allow for the examination of whether responses to items are SDO-specific, or if they are also (or alternatively) related to authoritarianism.

Chapter 4 focused on the multi-dimensional nature of SDO, generally finding that the anti-egalitarianism component of social dominance (SDO-E) is more strongly related to environmentalism than the dominance facet (SDO-D). I concluded Chapter 4 with a call for further research on whether certain aspects of environmentalism might be more closely aligned with SDO-D. This would be consistent with research in the prejudice domain, where SDO-E and SDO-D differentially predict intergroup attitudes (e.g. Ho et al., 2012, 2015). While Study 5A hinted at some differences in the strength of the correlations with different SDO facets, it did not have the power to control for each variable to examine unique associations using regression analysis. With a larger sample size in Study 5B, I pit the two dimensions against each other to test for differences in their predictive ability.

I also include a broader range of existing environmental measures to assess convergent validity. These measures ought to be related to scores on the interview-based 
items (and scale), assuming that all measure the same underlying construct (i.e. environmentalism). Therefore, I expect to find a positive association between proenvironmental interview-based items and climate change belief, pro-environmental attitudes, and willingness to make sacrifices for the environment.

To summarize, in Study 5B I build on the initial analyses of the piloted items. I do this by including a subset of the items from Study 5A and several other theoretically related scales, which allows for a more complete examination of the role of different ideologies and facets of ideology in a larger sample.

\section{Method}

\section{Participants}

Participants were 461 first year psychology students who took part in the survey in exchange for partial credit towards a mandatory research participation requirement. The majority of the sample were female $(\mathrm{n}=363)$, with a mean age of $19.11(S D=3.86)$. The ethics application from Study 5A (ID = 0000025633) was amended to secure approval for this study.

\section{Materials}

My measures were embedded in a longer survey with items tapping attitudes towards social, political, and scientific issues.

Ideology. I used the full 16-item version of Ho et al.'s (2012) SDO scale detailed in Chapter 4 Study 3B. This once again demonstrated good reliability $(\alpha=.86$; SDO-D: $\alpha=.74$; SDO-E: $\alpha=.80$ ). RWA was measured using Altemeyer's 30 -item scale as described in Chapter 3 Study 2A. I had intended to include Duckitt et al.'s (2010) ACT scale to allow for examination of associations with the three distinct dimensions of authoritarianism, however Altemeyer's scale was instead included in the survey by the broader research team. Therefore, examination of the role of RWA is limited to treating the construct as unidimensional $(\alpha=.92)$. I also included the foundations of these ideological attitudes: perceptions of the world as dangerous and competitive. I used Duckitt et al.'s (2002) scale, with ten items each to measure dangerous $(\alpha=.76)$ and competitive $(\alpha=.63)$ worldviews.

Existing environmentalism measures. The same reliable $(\alpha=.77)$ 12-item NEP scale described in Chapter 3 Study 2A measured pro-environmental attitudes. I included two 
items: 'climate change is real' and 'climate change is caused by humans' to measure belief in climate change $(r(459)=.69, p<.001,95 \%$ CI $[.77, .62])$. I also used the same sets of two items designed to measure both willingness to make $(r=.85, p<.001)$, and having made $(r=$ $.80, p<.001$ ), sacrifices for the environment as described in Chapter 3, Study $2 \mathrm{C}$.

Interview-based environmentalism items. To reduce the list of items from those included in the pilot study, I removed all items that were duplicates (same idea expressed in different wording), or demonstrated low inter-item correlations from Study 5A (i.e. $r$ 's between $+/$ - .2). Study 5B included 17 of the original 30 Likert-type items and 10 of the original 16 slider items. A complete list of the retained items is available in the Results section in Table 6.4 (Likert-scale items) and Table 6.5 (sliding scales), with the numbering consistent with Study 5A for ease of comparison.

\section{Procedure}

Participants completed the surveys across two sessions due to space constraints in each of the single sessions, with responses matched using student identification numbers. Partial participation credit was allocated separately for each survey completed. The data included in these analyses include only participants who completed both survey packets $(\mathrm{N}=$ 461).

\section{Statistical analysis}

I generally followed the same analytic procedure as in Study 5A. However, instead of presenting zero-order correlations with the items and SDO-E and SDO-D, I present beta weights from a linear regression. This pits the dimensions of SDO against each other as a test of which best explains the variance in each environmental variable (see Chapter 2).

\section{Results}

\section{Descriptive statistics of existing measures}

Table 6.3 includes means and standard deviations of the validated measures included in this study and correlations between these variables. These highlight an issue with endorsement of pro-environmental attitudes in this sample, as indexed by scores on the NEP. Specifically, scores were unrelated to all other environment-relevant variables and ideology, which is highly unusual. Examination of the univariate statistics reveals that scores on the NEP clustered tightly around the mean, with low variance ( $S D=.52$ on a 7-point scale). This 
could explain why correlations with other variables were so low, as there was little variance to explain in scores on the NEP (compared to the other variables). This means that using an association between the interview-based environmentalism items and the NEP as evidence of convergent reliability is flawed, given that the NEP is not performing as expected. I therefore include it only cautiously in further analyses. Descriptive statistics and correlations between other validated items fit with the literature (environmental items related, SDO and its dimensions related to these and other ideological variables). 
Table 6.3.

Descriptive information and correlations between mean scores.

\begin{tabular}{|c|c|c|c|c|c|c|c|c|c|c|c|}
\hline & Mean (SD) & 1. & 2. & 3. & 4. & 5. & 6. & 7. & 8. & 9. & 10. \\
\hline 1. SDO mean & $2.60(.82)$ & & & & & & & & & & \\
\hline 2. SDO-E & $2.47(.90)$ & - & & & & & & & & & \\
\hline 3. SDO-D & $2.74(.88)$ & - & $.70 * * *$ & & & & & & & & \\
\hline 4. RWA & $2.63(.77)$ & $.58 * * *$ & $.50 * * *$ & $.58 * * *$ & & & & & & & \\
\hline 5. CWV & $2.92(.68)$ & $.28 * * *$ & $.23 * * *$ & $.29 * * *$ & $.18 * * *$ & & & & & & \\
\hline 6. DWV & $3.87(.81)$ & $.18 * * *$ & $.13 * *$ & $.21 * * *$ & $.33 * * *$ & $.16^{* *}$ & & & & & \\
\hline 7. NEP & $4.41(.52)$ & -.01 & -.06 & .04 & -.02 & .04 & $.12 * *$ & & & & \\
\hline 8. CC belief & $6.09(1.06)$ & $-.26 * * *$ & $-.21 * * *$ & $-.26 * * *$ & $-.26 * * *$ & $-.12 *$ & $-.14 * *$ & $.11 *$ & & & \\
\hline 9. Will sacrifice & $5.45(1.21)$ & $-.36 * * *$ & $-.33 * * *$ & $-.33 * * *$ & $-.39 * * *$ & $-.22 * * *$ & $-.16^{* *}$ & $.12 *$ & $.32 * * *$ & & \\
\hline 10. Have sacrificed & $4.75(1.43)$ & $-.24 * * *$ & $-.24 * * *$ & $-.20 * * *$ & $-.28 * * *$ & $-.13 * *$ & $-.13 * *$ & .09 & $.21 * * *$ & $.71 * * *$ & \\
\hline 11. Enviro likert mean & $5.16(.70)$ & $-.40 * * *$ & $-.37 * * *$ & $-.37 * * *$ & $-.38 * * *$ & $-.27 * * *$ & $-.14 * *$ & $.20 * * *$ & $.38 * * *$ & $.62 * * *$ & $.55 * * *$ \\
\hline
\end{tabular}




\section{Planned analyses of Likert-scale items}

Table 6.4 presents the analyses of responses to the Likert-type items, with means and standard deviations presented after reverse coding of items. These follow the same pattern as Study 5A, with participants leaning, on average, towards pro-environmentalism. It also presents the correlations between the environmental variables and SDO, which show significant associations with every item. The lowest association is with Item 26 (Economic growth is bad for the environment), however this is still in the expected direction: stronger endorsement of SDO is linked to greater support for this item. Extending on these analyses, which broadly replicate the results of Study 5A, I examined the correlations with scores on the RWA scale. These generally match those with SDO, indicating that both ideologies are significantly related to the environmental items.

The table also includes standardized regression coefficients with SDO-E and SDO-D, showing that a similar number of items are more strongly predicted by SDO-E as those more strongly predicted by SDO-D. For many items, only one facet of SDO is a significant predictor. For instance, only SDO-E is associated with decreased support for sacrificing money (item 1) and effort (item 2) for the environment, as well as endorsing the belief that technology will solve climate change (item 27). In contrast, SDO-D is the only predictor of items suggesting businesses should not have to make sacrifices (items 24, 29 and 30).

\section{Exploratory analyses of Likert-scale responses}

The exploratory analyses of these items from Study 5A showed that they work reasonably well together as a coherent scale, with good internal reliability. However, the reliability analyses may have been artificially inflated given that several items were written to tap the same idea with variations on the wording. The updated scale with reduced items included in Study 5B avoids this problem. The pilot study did show some convergent and discriminant validity, but there were few variables included in the dataset, and therefore a full examination of this was not possible. Here, I repeat the psychometric evaluation of the Likert-scale responses, and examine convergent validity with climate change belief and support for sacrificing for the environment.

Step 1. I reverse coded relevant items so that all pointed in the pro-environmental direction. Again, I initially did not recode items 11, and 26, which arguably (and supported by analyses in Study 5A) are nondirectional, and items 22 and 25 (appear anti-environmental, but limited sample from Study 5A pointed to pro-environmental) to let the internal reliability 
analyses point to whether they ought to be included and reverse coded (data driven approach).

Step 2. To determine whether the items did indeed form a unidimensional scale, as I speculated in Study 5A, or if there is an underlying multidimensional factor structure, I conducted an exploratory factor analysis. Because of the small sample size and relatively large number of variables included in Study 5A, I could not do this step, as a variables to cases ratio of less than 1:5, when it should be over 1:20, with ideally over 300 participants in total (Comrey \& Lee, 1992). The variables:cases ratio in this study is about 1:30. I started by including all 17 variables, and used a Principal Components model to analyse all variance in the variables.

Four factors with Eigenvalues above 1 were extracted, with the first explaining the majority of the variance $(27.11 \%$, Eigenvalue $=4.61)$, with a clear drop in the Scree plot and variance explained between this and factor 2 , which explained $10.62 \%$ of the variance $($ Eigenvalue $=1.82)$. Below that, factors 3 and 4 explained similar amounts of variance (7.27\% and 6.37\%, Eigenvalue's $=1.24,1.09$, respectively). These observations support the interpretation of the scale as unidimensional, however the rotated solution (using Oblimin to allow for associations between extracted factors) showed that items loaded on three distinct factors, with few cross-loadings above .3. I removed one complex item (Item 20, as it had a primary loading of .49, and a cross loading of -.39), and one item with low communalities (.12, Item 26), and the resulting solution gave a clearer three factor structure. The three factors explained $48.11 \%$ of the variance in the 15 items, with Eigenvalues of 4.25 (explaining $28.33 \%$ of the variance), $1.76(11.73 \%)$, and $1.21(8.05 \%)$. 
Table 6.4.

Rotated factor matrix.

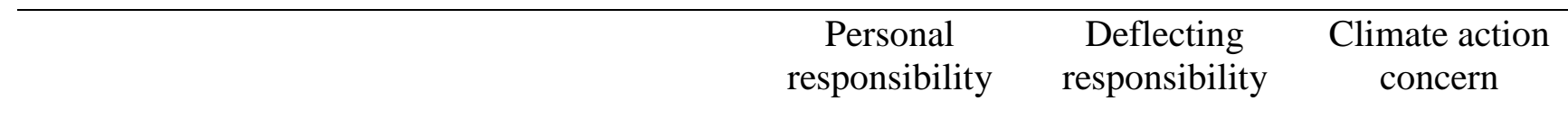

I'm happy to spend more on a product if it's environmentally friendly

I won't do an environmentally friendly action if it's going to take a lot more effort

I feel personally responsible for finding solutions to climate change

We should try to change our lifestyles in order to reduce our impact on the environment

Individuals shouldn't be expected to make changes to their lifestyles for the environment

The best way for individuals to help the environment is to put pressure on the government to make changes from above

Polluting industries are responsible for finding solutions to climate change

The government is responsible for finding solutions to climate change

The government should introduce incentives to promote environmentally friendly practices

The government should introduce fines to reduce environmentally unfriendly practices

It is more important for companies to prioritise minimizing their environmental impact than making profits

Businesses shouldn't have to worry about their environmental impact: The government should stay out of it

We don't need to worry about climate change, because new technologies will eventually solve our environmental problems

Acting on climate change is going to cost more than not acting

I would be embarrassed if my friends thought I was becoming an 'environmentalist'

Note. Factor loadings below .3 have been suppressed. 
The first five items in Table 6.4 cluster on the first factor, and appear to sensibly be measuring felt personal responsibility for action. The second six set of items cluster on the second factor, which centers on deflecting personal responsibility (e.g. onto government). The final four items load onto the third factor, which appears to measure climate action worries (i.e. denying that we should be worried about the environment, and worrying instead about the economic or social costs of action).

To see if these three factors were reliable, I computed alphas for each. Personal responsibility $(\alpha=.74)$ and deflecting responsibility $(\alpha=.70)$ factors were reliable, however the climate action concern factor was not $(\alpha=.55)$. Removal of Item 3 , which had a low corrected item-total correlation (.21) saw the reliability increase to an acceptable level for a three-item scale $(\alpha=.66)$. In Table 6.5 below, I include descriptive information and correlations between these factors. These demonstrate moderate correlations between the factors themselves, and that each factor is similarly reated to both SDO and RWA.

Table 6.5.

Descriptive information and correlations between environmentalism scale factors and SDO.

\begin{tabular}{|c|c|c|c|c|c|}
\hline & 1. & 2. & 3. & 4. & 5 . \\
\hline $\begin{array}{l}\text { 1. Personal } \\
\text { responsibility }\end{array}$ & $5.06(0.94)$ & & & & \\
\hline 2. Deflecting & .43 & $4.96(0.80)$ & & & \\
\hline responsibility & {$[.34, .51]$} & & & & \\
\hline 3. Climate & .48 & .32 & $5.85(0.95)$ & & \\
\hline action concern & {$[.41, .56]$} & {$[.24, .40]$} & & & \\
\hline 4. SDO & -.34 & -.30 & -.32 & - & \\
\hline \multirow{3}{*}{ 5. RWA } & {$[-.43,-.25]$} & {$[-.39,-.20]$} & {$[-.40,-.24]$} & & \\
\hline & -.30 & -.29 & -.33 & .58 & - \\
\hline & {$[-.38,-.20]$} & {$[-.38,-.19]$} & {$[-.41,-.24]$} & {$[.51, .65]$} & \\
\hline
\end{tabular}

Note. In the diagonals are the subscale means and (standard deviations). 95\% confidence intervals are based on 1000 iterations. SDO = social dominance orientation, RWA $=$ right-wing authoritarianism. 
As a final exploration of how the factors are associated with SDO, I entered the two components (SDO-D and SDO-E) into a multiple linear regression. These analyses show that SDO-E is more strongly related to feelings of personal responsibility (SDO-E: $\beta=-.27, p<$ .001 , SDO-D: $\beta=-.10, p=.116$ ), however SDO-D is more strongly related to deflection of responsibility (SDO-D: $\beta=-.26, p<.001$, SDO-E: $\beta=-.06, p=.308$ ), while the dimensions are similarly related to climate action concern (SDO-E: $\beta=-.19, p=.002$, SDO-D: $\beta=-.16, p$ $=.013)$.

Step 3. I also checked how the scale performed overall. The Cronbach's alpha of the 17 items indicated acceptable internal reliability $(\alpha=.80)$. To see how the scale could be improved, I next examined the corrected item-total correlations. These pointed to two items with very low associations with the overall scale (below .1). Items 3 and 26 ("Acting on climate change is going to cost more than not acting' and 'Economic growth is bad for the environment') were excluded, and the analyses repeated. The alpha improved $(\alpha=.83)$, and examination of the items with unclear wording showed they all correlated positively with the rest of the scale. The corrected item-total correlations ranged from .31 to .56 , verifying that no remaining items needed reverse coding or removing (therefore items 22 and 25 loaded positively in this scale, indicating they are pro-environmental). I computed a mean score for each participant on the 15-item scale.

Step 4. Univariate statistics for the overall 15-item scale again showed that participants leant towards pro-environmentalism $(M=5.16, S D=.70)$.

Step 5. In Table 6.6, I present correlations between mean scores on the Likert-based items, other indices of environmentalism, and ideological variables. These analyses reveal significant positive associations between my environmental mean and the other indices of pro-environmentalism, and therefore demonstrate convergent validity.

Mean scores on the Likert-based items also exhibited moderate negative associations with SDO, RWA, and the separate dimensions of SDO. Scores were also significantly related to worldview, with perceptions of the world as a dangerous relating to lower proenvironmental responding. Similarly, the view of the world as a competitive place (the worldview foundational for SDO; Duckitt, 2001) related to lower pro-environmental responding. 


\section{Planned analyses of sliding scale responses}

For the sliding scale analyses presented in Table 6.7, the means show the same pattern of responses as in Study 5A, with participants on average leaning towards the environmentally friendly objective. Items 5, 6, 11, and 12 are unrelated to mean scores on the SDO scale, while the remaining six items are. Eight items are significantly related to RWA, which show that while SDO is unrelated to placement on how harmful or helpful economic growth is, individuals endorsing RWA tend to believe economic growth is more helpful for the environment than those low in RWA. Similarly, while SDO is a non-significant correlate, RWA predicts support for individuals as responsible for pollution, for placing the cost on individuals buying products (through taxes), while individuals low in RWA place blame to a greater extent on industry, and prefer industry to be taxed. Regarding the short-term economic effects of acting on climate change, endorsement of SDO predicts placement closer to negative effects, while RWA is unrelated. However, both are related to perceiving more negative effects long-term than low-RWA and low-SDO individuals.

The zero-order correlations in Study 5A showed that SDO-E and SDO-D were differentially related to the items such that a roughly equivalent proportion of items were more strongly related to each facet. The analyses presented here control for each component to examine the unique association each has with the items. Two items show a clear differentiation between the facets: Item 11 and 12, which could explain the nonsignificant associations between overall endorsement of SDO and these items. In item 11, SDO-E relates negatively (in the direction towards favouring taxing environmentally unfriendly industries; $\beta$ $=-.109, p=.094)$, while SDO-D relates positively, in the direction of applying costs to environmentally unfriendly products $(\beta=.178, p=.006)$. Similarly, item 12 shows divergence between the facets with SDO-E relating negatively (thus in the direction of preferring the government to find solutions to climate change, $\beta=-.161, p=.013$ ), while SDO-D relates positively (thus in the direction of preferring individuals to find the solution, $\beta$ $=.214, p=.001)$.

The direction (positive or negative) of the associations reveal which objective individuals high in SDO-D and E lean towards, this is relative to those low in SDO-D and E. Therefore, they could be placing themselves anywhere along the sliding scale, and without plotting average endorsement (as in the analyses of Study $5 \mathrm{~A}$ ), it is not possible to tell where high- and low-SDOs are really placing themselves on the scale. 
Table 6.6.

Descriptive analyses of Likert-scale response items, including associations with SDO.

\begin{tabular}{|c|c|c|c|c|c|}
\hline Environmental attitude item & Mean (SD) & $\begin{array}{c}r \\
\text { RWA }\end{array}$ & $\begin{array}{c}r \\
\text { SDO }\end{array}$ & $\begin{array}{c}\beta \\
\text { SDO-E }\end{array}$ & $\begin{array}{c}\beta \\
\text { SDO-D }\end{array}$ \\
\hline 1. I'm happy to spend more on a product if it's environmentally friendly & $5.15(1.31)$ & $-.187 * * *$ & $-.220 * * *$ & $-.247 * * *$ & .009 \\
\hline 2. I won't do an environmentally friendly action if it's going to take a lot more effort (R) & $5.02(1.44)$ & $-.168 * * *$ & $-.255^{* * *}$ & $-.197 * *$ & -.079 \\
\hline 3. Acting on climate change is going to cost more than not acting $(\mathrm{R})$ & $4.68(1.95)$ & $-.166 * * *$ & $-.153 * *$ & -.047 & $-.119^{+}$ \\
\hline $\begin{array}{l}\text { 6. We should try to change our lifestyles in order to reduce our impact on the } \\
\text { environment }\end{array}$ & $5.89(1.00)$ & $-.274 * * *$ & $-.304 * * *$ & $-.187 * *$ & $-.142^{*}$ \\
\hline $\begin{array}{l}\text { 11. The best way for individuals to help the environment is to put pressure on the } \\
\text { government to make changes from above (X) }\end{array}$ & $4.81(1.30)$ & $-.217 * * *$ & $-.229 * * *$ & $-.119^{+}$ & $-.130 *$ \\
\hline 15. I feel personally responsible for finding solutions to climate change & $3.96(1.52)$ & $-.197 * * *$ & $-.173 * * *$ & $-.135^{*}$ & -.052 \\
\hline $\begin{array}{l}\text { 17. I would be embarrassed if my friends thought I was becoming an 'environmentalist' } \\
\text { (R) }\end{array}$ & $5.67(1.36)$ & $-.212 * * *$ & $-.233 * * *$ & $-.195 * *$ & -.058 \\
\hline $\begin{array}{l}\text { 20. The government should prioritise the environment, even if this is at the expense of } \\
\text { the economy }\end{array}$ & $4.77(1.27)$ & $-.220 * * *$ & $-.181 * * *$ & $-.117^{+}$ & -.078 \\
\hline $\begin{array}{l}\text { 21. Individuals shouldn't be expected to make changes to their lifestyles for the } \\
\text { environment }(\mathrm{R})\end{array}$ & $5.28(1.38)$ & $-.237 * * *$ & $-.258 * * *$ & $-.186 * *$ & -.094 \\
\hline 22. The government is responsible for finding solutions to climate change (R) & $3.42(1.37)$ & $.109 *$ & $.139 * *$ & $.101 *$ & $.156^{* *}$ \\
\hline $\begin{array}{l}\text { 23. The government should introduce fines to reduce environmentally unfriendly } \\
\text { practices }\end{array}$ & $5.11(1.31)$ & $-.250 * * *$ & $-.216 * * *$ & $-.115^{+}$ & $-.119^{+}$ \\
\hline 24. The government should introduce incentives to promote environmentally friendly & $5.94(1.00)$ & $-.281 * * *$ & $-.233 * * *$ & -.018 & $-.235 * * *$ \\
\hline
\end{tabular}
practices 
25. Polluting industries are responsible for finding solutions to climate change (R)

26. Economic growth is bad for the environment $(\mathrm{X})$

27. We don't need to worry about climate change, because new technologies will eventually solve our environmental problems $(\mathrm{R})$

29. It is more important for companies to prioritise minimizing their environmental

30. Businesses shouldn't have to worry about their environmental impact: The government should stay out of it (R) impact than making profits

$\begin{array}{lllll}3.34(1.30) & .128 * * & .150 * * & .104 * & -.173 * * \\ 3.61(1.20) & .073 & .098 * & .102 & .005 \\ 5.91(1.18) & -.230 * * * & -.237 * * * & -.177 * * & -.080 \\ & & & & \\ 4.64(1.33) & -.158 * * & -.195 * * * & -.038 & -.173 * * \\ 5.96(1.16) & -.330 * * * & -.271 * * * & -.060 & -.233 * * *\end{array}$

Note. For ease of comparison with the Study 5A results, I have kept the numbering of items consistent, and present these results prior to reverse coding of the variables. 
Table 6.7.

Descriptive analyses of sliding scale response set items, including associations with SDO.

\begin{tabular}{|c|c|c|c|c|c|}
\hline & Mean (SD) & $r$ RWA & $r \mathrm{SDO}$ & $\beta$ SDO-E & $\beta$ SDO-D \\
\hline 1. Government: The economy - The environment & $59.97(17.63)$ & $-.287 * * *$ & $-.197 * * *$ & .012 & $-.226 * * *$ \\
\hline 4. Minerals: The investors - The community & $60.34(18.74)$ & $-.249 * * *$ & $-.201 * * *$ & $-.132 *$ & -.086 \\
\hline 5. Pollution: Industries - Individuals & $38.55(24.26)$ & $.112 *$ & .075 & -.002 & .084 \\
\hline $\begin{array}{l}\text { E. Economic growth: Harmful for the environment - Helpful for the } \\
\text { environment }\end{array}$ & $40.63(17.77)$ & $.147 * *$ & .053 & -.086 & $.143^{*}$ \\
\hline 8. Industry: Making profits - Minimizing environmental impact & $64.20(16.88)$ & $-.241 * * *$ & $-.206^{* * *}$ & -.041 & $-.182 * *$ \\
\hline $\begin{array}{l}\text { 9. Short term: Acting has Negative effects of the economy - Positive } \\
\text { effects on the economy }\end{array}$ & $50.27(21.23)$ & -.042 & $-.116^{*}$ & $-.126^{+}$ & .000 \\
\hline $\begin{array}{l}\text { Taxes: Environmentally unfriendly industries - The cost of } \\
\text { environmentally unfriendly products }\end{array}$ & $42.34(23.46)$ & $.119^{*}$ & .064 & -.109 & $.178^{* *}$ \\
\hline 12. Responsibility: The government alone - Individuals alone & $45.73(15.04)$ & .027 & .049 & $-.161 *$ & $.214 * *$ \\
\hline $\begin{array}{l}\text { 15. Long term: Acting has Negative effects on the economy - Positive } \\
\text { effects on the economy }\end{array}$ & $67.94(20.12)$ & $-.177 * * *$ & $-.186 * * *$ & .010 & $-.212 * *$ \\
\hline 16. Cause: $\mathbf{1 0 0 \%}$ human activity $-100 \%$ natural causes & $24.54(17.58)$ & $.187 * * *$ & $.139 * *$ & -.032 & $.183^{* *}$ \\
\hline
\end{tabular}




\section{Exploratory analyses of sliding scale responses}

Next, I analyse results by splitting participants by SDO-level (overall) based on a quartile split, as used in Chapter 5. While in Study 5A I examined the difference between high- and low- scorers based on a mean split, the larger sample size of the current study would allow for a finer examination of how differing SDO-levels relate to endorsement of these items. Therefore, I created three groups using the same method as was used in Study 4 (low, moderate, which included low-moderate and high-moderate, and high, consistent with the interview study).

Table 6.8 shows how the samples were split, with the cut-off criteria (based on the quartile split), sample size in each group, and mean levels of SDO. I used One-Way ANOVAs to verify that levels of SDO were significantly different across the newly created groups $(F(2,458)=1159.60, p<.001)$, with each group significantly different from the other (all $p$ 's $<.001)$.

Table 6.8.

Descriptive information about the three groups based on SDO level.

\begin{tabular}{llll}
\hline & Low-SDO & Moderate-SDO & High-SDO \\
\hline SDO & & & \\
\hline Cut off points & $0-1.9375$ & $1.9376-3.2499$ & $3.2500-7$ \\
$\mathrm{~N}$ & 119 & 223 & 119 \\
Mean (SD) & $1.58(.27)$ & $2.58(.36)$ & $3.67(.34)$ \\
\hline
\end{tabular}

Table 6.9 presents the results of a series of One-Way ANOVAs testing for differences in where high- moderate- and low-SDO individuals place themselves on the sliding scales. Seven of the ten associations differed by SDO level, and most differences are between highand low-SDO groups, which suggests the relationship between environmentalism and ideology is linear.

To guide interpretation of these findings, Figure 6.3 shows the average placement on the sliding scales per group. In these graphs, to better visualize the associations, the opposing adjectives are at either extreme end (as 50 and -50), and the midpoint (0) represents the middle of the sliding scale (marked 'both equally'). 
Table 6.9.

Group-based differences in sliding scale responses.

Item

One-way ANOVA

Interpretation

1. Govt: The economy - The environment

4. Mining: The investors - The community

5. Contributors: Industries - Individuals

6. Economic growth: Harmful for the environment Helpful for the environment

8. Companies prioritize: Making profits - Minimizing environmental impact

9. Action short term: Negative effects of the economy -

Positive effects on the economy

11. Tax: Environmentally unfriendly industries - The cost of environmentally unfriendly products

12. Responsibility for solutions: The government alone Individuals alone

15. Acting long term: Negative effects on the economy Positive effects on the economy

16. Cause: $100 \%$ human activity $-100 \%$ natural causes
$\mathrm{F}(2,458)=5.22, p=.006 \quad$ Low and high groups significantly different

$\mathrm{F}(2,458)=7.82, p<.001$

Low and moderate groups significantly different from high group

$\mathrm{F}(2,458)=4.85, p=.008 \quad$ Low and high groups significantly different

$\mathrm{F}(2,458)=.08, p=.924 \quad$ No differences between groups

$\mathrm{F}(2,458)=8.37, p<.001$

Low and moderate groups significantly different from high group

$\mathrm{F}(2,458)=3.07, p=.047 \quad$ Low and high groups significantly different

$\mathrm{F}(2,458)=1.90, p=.150 \quad$ No differences between groups

$\mathrm{F}(2,458)=.92, p=.400 \quad$ No differences between groups

$\mathrm{F}(2,458)=7.03, p=.001 \quad$ Low and high groups significantly different

$\mathrm{F}(2,458)=4.09, p=.017$ 


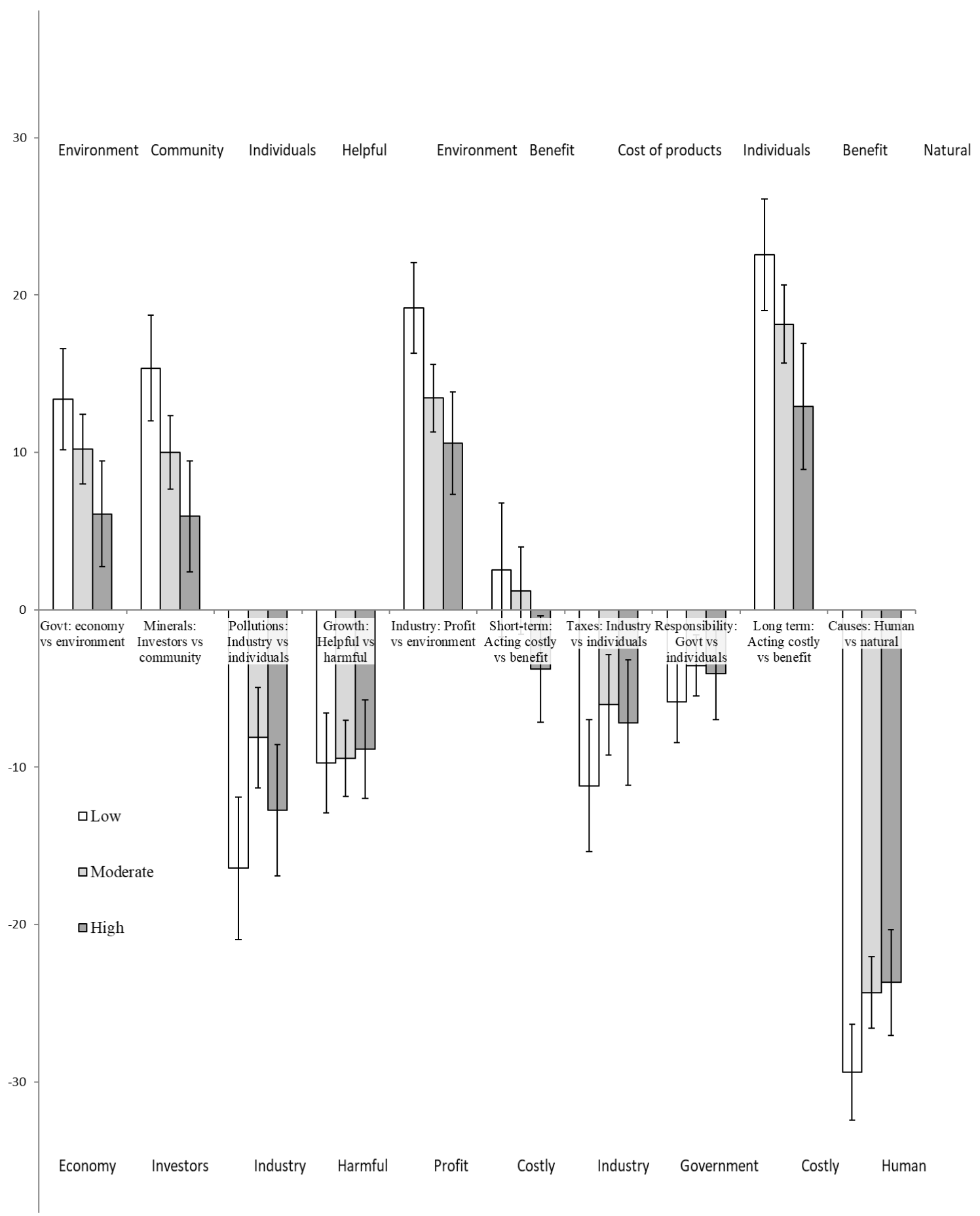

Figure 6.3. Placement on sliding scale by SDO group.

Note. Anchors are at 50 and -50, midpoint signifies endorsement of each objective equally.

Figure 6.3 depicts the pattern of results, where individuals higher in SDO generally place their opinion further from the pro-environmental objective than individuals lower in SDO. For instance, individuals endorsing low levels of SDO think the government should pay relatively greater attention to the environment than the economy, but high-SDOs also respond favouring the environment, just to a lesser extent. Indeed, high-SDOs even lean 
towards giving the community a share of the resources from a mine, again to a lesser extent than low-SDOs. In Study 5B, the only instance where high- and low-SDOs respond in different directions is for the short-term effects of acting on climate change, though the group differences were not significant. Low-SDOs leaned towards there being an economic benefit of acting (though this crosses the midpoint, suggesting they generally endorse the "both equally' response), while high-SDOs lean towards short-term action being costly. For longterm action, low-SDOs saw significantly more economic benefit to acting on climate change, and while high-SDOs agreed there would be benefit, they did so to a lesser extent. These graphs reveal more clearly that high-SDOs are in actuality on the same 'side' as low-SDOs.

However, recall that the 'high-SDO' group still on average scores below the midpoint on the scale. That is, they are still on average opposing inequality and intergroup dominance. Consideration of the histogram of SDO scores shows that no individuals score above 5 ('agree') on the 7-point scale, with only $4.1 \%$ scoring above the scale midpoint. This indicates that participants are overwhelmingly against the ideas captured by the scale of inequality and dominance.

\section{Comments}

In Study 5B, I presented a subset of the items already piloted in Study 5A to a larger group of participants. I found that in general, participants leant towards endorsing proenvironmentally-worded items. Importantly, this was even the case for (relatively) highSDOs. It is not that Social Dominants endorsed anti-environmentalism, as could be interpreted from the negative correlations, but instead they may be pro-environmental, just to a lesser extent than their low-SDO counterparts. Furthermore, participants who were deemed high-scorers on the SDO scale similarly rejected inequality and intergroup dominance, though not to the same degree as low-SDOs.

Indeed, scores on the SDO scale suffered from a form of range restriction, with participants almost exclusively scoring on average below the midpoint of the scale. This is potentially problematic, because I did not capture any individuals who express a preference for inequality and dominance. The 'high-SDOs' are therefore not high endorsers of SDO at all, they are indifferent: neither agreeing nor disagreeing with the items. Perhaps they are therefore best described as tolerant of social dominance and inequality, or apathetic towards it, rather than endorsers. I consider how this finding affects interpretation of the research in this area in the General Discussion (Chapter 7). 
Study 5B expanded on Study 5A by incorporating RWA, and using this to show that the items were not necessarily specific to SDO, but instead associated with both ideologies. This is consistent with Study 1A, which revealed similar correlations between SDO, RWA, and environmental measures. In the Discussion on the next page, I draw conclusions from across the two studies described in this chapter. 


\section{Discussion of Studies 5A and 5B}

The purpose of the studies in this Chapter was to present a larger group of people with the ideas shared by interviewees in Chapter 5 to determine whether the ideas (as reformatted into survey items) were generally supported by a larger group of people, and if they were associated with ideology. Indeed, I found that participants across my two studies generally responded in the pro-environmental direction (where such a direction could be determined), agreeing with many of the concerns, suggested solutions, and perceived barriers as were expressed in the interviews. Responses were also tied to participant's ideological stances as expected.

In Study 4, I did not find associations between participant's SDO level and the ideas they shared about climate change in their interviews. This suggests that the absense of a mention of a theme by the interviewees does not mean they do not endorse that theme, but simply that they did not mention it. Here, when presented with each idea, the difference in low- and high-SDO's opinions emerge. To reconcile apparent differences across Chapters 5 and 6, this suggests that individuals opinions are different and associated with ideology, but they are as adept at recognising different arguments about climate change.

In these studies, endorsement of SDO was related to greater sensitivity to the cost and effort associated with pro-environmental action through lower agreement with these items. While individuals scoring relatively higher on the SDO scale were less likely to feel personal responsibility for climate change, they were also more opposed to government- or businesslevel action on climate change. The only 'solution' they were more supportive of involved technology to solve the problem.

It is therefore possible that Social Dominants' consistently lower pro-environmental responding is an expression of a general underlying environmental attitude. This would be consistent with the findings from Chapter 2 , which revealed roughly equivalent associations between SDO and different indices on environmentalism. Indeed, I found that the items combined to form a scale, and endorsement of SDO was significantly related to lower mean endorsement of the environmental items. Higher scores on RWA were similarly related to lower endorsement of these items. This scale could also be separated into three factors, which differentially related to the dimensions of SDO.

In Chapter 4, I demonstrated that SDO-E is more strongly related to environmentrelated items than SDO-D, concluding with a call for future research exploring whether SDO- 
D was more strongly related to other facets of environmentalism. Combined, the dimensions of SDO relate to lower environmentalism overall, however an exploration of how the two dimensions relate to a wider range of environment-relevant issues would help to further disentangle the SDO-environmentalism association. An SDO-D interpretation of the SDOenvironmentalism link would perhaps suggest that the relationship exists due to the preference for human dominance over nature (e.g. Milfont et al., 2013), while an SDO-E interpretation supports the idea that humans use the environment to enhance status differences between groups (e.g. Jackson et al., 2013; Milfont \& Sibley, 2014). On examination of the associations with the three subscales in this study, I note there is a clear pattern where SDO-D is related to greater resistance to government or industry-level action on climate change (i.e. action that deflects responsibility onto government and business, similar to the Solutions theme: public pressure, top-down solutions), while SDO-E is more strongly related to opposition to engaging in personal action on climate change (opposing personal responsibility, similar to the Hopeless Environmentalist theme). Both SDO-D and SDO-E relate to concern about action on climate change, which broadly maps onto aspects from all three main themes from Study 4.

High-SDO-Ds support the dominance of high-status groups over subordinate groups. In the intergroup relations context, these individuals are more aggressive and exhibit overt racism (Ho et al., 2012). They are more active oppressors of low-status groups, compared to high-SDO-Es, who are more covert in their resistance to moves towards equal intergroup relations. At first glance, the pattern of associations are surprising in light of Ho et al.'s (2012) findings that SDO-E is more predictive of policy support (when this would enhance or maintain hierarchy, e.g. symbolic racism, system legitimacy) and opposition (for those that would reduce inequality, e.g. affirmative action or social welfare). Top-down solutions to climate change are generally policy measures, however in this case SDO-E is largely unrelated to these items, while SDO-D is a significant predictor of relatively lower support.

SDO-E endorsers are unphased by appeals to equalise conditions for others. Instead, they tolerate differences in quality of life across groups. Why then, would they make personal sacrifices to their own quality of life for the environment? Aside from the SDO-E-policy distinction, the dimensions differ on how overt the attitudes and behaviours they predict are. Individual pro-environmental actions are analagous to the subtle forms of prejudice, as they are less visible. For instance, people will not necessarily know if others are taking personal actions for the environment, such as reducing waste or conserving power. The conclusion 
from Chapter 4 that the SDO-environmentalism association exists because Social Dominants attempt to secure disproportionate access to natural resources for high-status groups still stands with these new findings. Resistance to engaging in personal actions for the environment means securing more resources for oneself, through making no sacrifices to personal finances, time, effort, or consumption. Government or business action, however, represents a different category of action on climate change.

Those who endorse SDO-D favour more overt forms of aggression directed at lowstatus groups, power structures that allow high-status groups to subjugate others, and using more forceful approaches to achieve such inequality than SDO-E. SDO-D is an ideology more sensitive to threat (Ho et al., 2015), which could explain their opposition to top-down solutions to climate change, as these tend to be viewed as presenting a threat to both the current way of life and economy (Clarke, Ling, Kothe, Klas \& Richardson, 2019; Hoffarth \& Hodson, 2016). SDO-D is also more strongly related to nationalism (Ho et al., 2015). Recall that Peterson et al. (1993) showed RWA related to the belief that environmental action would reduce ones country to a second-rate power. Social dominants might also be sensitive to this idea, given their relatively stronger fear of economic threats and perception that the world is a competitive place (Duckitt, 2001). The sensitivity to country-level economic success, coupled with greater nationalism and therefore the desire for one's own country's superiority, could explain why high-SDO-Ds are more opposed to government and business action on climate change. Furthermore, top-down support for environmental exploitation (by way of rejecting such actions) is particularly overt, sending a clear message that human dominance over nature is permissible.

As endorsement of SDO-D was largely unrelated to opinions on personal actions, these might be more palatable to high-SDO-Ds than top-down action. In constrast, highSDO-Es are more opposed to personal actions, and therefore could be more likely to support top-down actions if prompted. The findings could be applied to help tailor climate change communications to target those who endorse hierarchical thinking, without 'wasting' time and effort attempting to promote potential solutions that are in direct conflict with individual's ideological stances. My findings would need to be replicated, ideally in a general population sample, to verify that SDO-E and SDO-D are indeed better predictors of different kinds of environmental action, and ideally efforts to motivate action ought to be tested experimentally. 
In sum, the findings from this chapter suggest that it is not SDO-E solely and uniformly predicting lower pro-environmentalism than SDO-D, but instead the two dimensions may be related to different outcomes. The items based on ideas from the interview study were pilot tested and then administered to a larger sample from the same group as the interviewees, which is one strength of this work. However, one limitation is that student samples tend to exhibit a different relationship between SDO and environmentalism (Chapter 2). Future work is needed to see whether these findings hold in a general population study. 


\section{Chapter 7}

\section{General discussion}

In this thesis, I present a series of ten studies with the overarching aim of clarifying the nature of the association between social dominance orientation (SDO) and environmental beliefs, attitudes and behaviours. In this chapter, I summarize my key findings and contributions to the literature. I also explore the main theoretical implications, limitations, and future directions for research into attitudes on the environment and climate change.

\section{Key contributions to the literature}

My original contribution to research on the environment-ideology link began in Chapter 2 by surveying the literature to date on the topic and using meta-analysis to combine effect sizes (Stanley \& Wilson, 2019). This demonstrated that SDO is a key correlate of a range of attitudes towards the environment and climate change, related to a similar degree as RWA. I show that each ideological attitude has a significant and independent association with environmentalism. Importantly, I also show that the relationship between SDO and environmentalism is moderated by sample type, with stronger associations in the general population than in student samples.

While the meta-analyses confirm that there is a growing interest and body of research on the relation between SDO and environmentalism, it does little to implicate SDO as causally related to attitudes towards the environment and climate change. In Chapter 3, I conducted two studies examining how SDO and RWA relate to environmentalism over time (Stanley et al., 2017a; Stanley et al., 2019). The first showed that RWA and not SDO prospectively predict increases in environmentalism. Following this up using a general population sample surveyed over five years, the opposite pattern of results emerged. Instead, both ideologies related to decreases in environmentalism, however SDO was a statistically stronger predictor. This is consistent with the weaker association in student samples, and suggests that this 
moderation by sample type also occurs across time: For those in the general population, attitudes about inequality are more strongly tied to changes in environmental attitudes over time. However, in terms of the temporal direction of the SDO-environmentalism link, I showed that environmentalism is a stronger predictor of SDO than the reverse.

Having established that SDO is related to environmentalism, both within and across time points (at least for general population samples), I then shifted focus to the theoretical foundations of SDO. Specifically, recent advancements confirm conceptualizing SDO as comprised of two subfactors: One favouring intergroup dominance, and the other anti-egalitarianism. In Chapter 4, using a series of three studies, I show that the SDO-environmentalism association is driven by the preference for inequality (anti-egalitarianism) to a greater extent than dominance (Stanley et al., 2017b).

In Chapter 5, I presented thematic analysis from semi-structured interviews of individuals with low, moderate, and high levels of SDO. These analyses revealed that many participants express fear over the dangers of climate change and have some ideas about how to address the problem, though also tend to be armed with barriers that justify their inaction. Many of these themes are consistent with previous qualitative research, but there was little evidence that mentioning these themes was associated with interviewee levels of SDO. Taking the main ideas (codes and themes) from this study, I created quantitative survey items to gauge agreement in a larger sample (Chapter 6). This research confirmed that a larger group generally agrees with pro-environmental items, even those scoring relatively higher in SDO. However, relatively higher levels of SDO were associated with relatively lower proenvironmentalism, as expected. Opposition to climate change solutions were also differentiated by the dimension of SDO, with dominance relating to greater opposition to Top-Down solutions to climate change, while anti-egalitarians were more opposed to individual actions.

In each successive Chapter, I try to advance knowledge on the SDOenvironmentalism link further: by being the first to examine the relationship metaanalytically, across time, using the two-dimensional model of SDO, and using a 
mixed-methods approach. In the next section, I discuss how these advancements relate to broader work on environmental psychology and intergroup relations.

\section{Implications and applications}

My research strengthens the utility of viewing environmental issues and climate change through the lens of an environmental justice framework. Both on a global and local level, there is an inequitable distribution of benefits and consequences of environmental utilization. Higher status groups typically enjoy a greater share of environmental resources than low status groups, live lifestyles that emit greater quantities of greenhouse gasses, yet will be relatively unscathed by the negative effects of climate change (IPCC, 2014b). Based on these observations, it makes sense then that those individuals who are relatively more tolerant of inequality (i.e. hold a stronger social dominance orientation) will be less concerned about reducing their impact on the environment.

Jylhä (2016) first made this link in her thesis and empirical work (Jylhä et al., 2015, 2016), using climate justice as one potential explanation of the SDOenvironmentalism association. With a main focus on climate change denial, Jylhä and colleagues assert that individuals who endorse SDO are motivated to deny climate change as a way of ensuring they will not have to make changes to their lifestyles. This interpretation positions Social Dominants as motivated to protect the current system of group-based hierarchy, where the higher status group members continue to enjoy environmental utilization with little consequence. This fits with earlier ideas about high-SDOs as motivated to reject environmentalism in order to secure disproportionate access to natural resources for high-status groups (Milfont \& Sibley, 2014), while directing the environmental consequences of this to lower-status groups (Jackson et al., 2013). It is also consistent with SDO-E being generally more strongly related to environmentalism than SDO-D, with anti-egalitarianism an ideology that appears to permit selfish use of the environment. Stronger feelings of resentment towards racial minorities is associated with climate change denial, a finding which Benegal (2018) has labelled evidence of the spillover of racialization. Drawing together environmental and social concerns, Klinsky (2017, p. 4) points out that "justice, and its flipside injustice, are central to the intersection of climate change and human well-being, and to political systems at all levels". 
Human interactions with the natural environment are an example of a tragedyof-the-commons (Gardner \& Stern, 1996): Individuals benefit from taking a competitive approach through using more natural resources for themselves or their own group. This is at odds with the best interests of the broader community, which benefits most from cooperation, where use of the environment is restricted. Those more concerned with the interests of their ingroup, and relatively less concerned with the interests of the collective or all groups, will compete. Endorsement of SDO is an expression of the motivational goals of superiority and dominance, spurred by a view of the world as a competitive place (Duckitt, 2001), therefore permitting exploitation of natural resources. Meanwhile, those rejecting inequality strive towards an egalitarian use of nature (i.e. cooperation/restriction), hence explaining why lower endorsement of SDO is related to pro-environmentalism.

This has concerning implications in the current political climate. Beyond SDO stifling individual engagement in environmentalism, it is also related to lower support for top-down initiatives, such as global climate change agreements (see Chapter 6). Mitigating climate change requires countries to cooperate to reduce greenhouse gas emissions. However, in less egalitarian societies, and in those where the leadership is more conservative, top-down action is hindered. At the time of writing, one example of this is the United States, which is currently reneging on promises made at the Paris accord (Saad, 2018). For those with a strong social dominance orientation, action on climate change is perceived as threatening the social and economic success of one's country (Clarke et al., 2019; Hoffarth \& Hodson, 2016). The politicization of climate change has meant that there are clear ideological divides between liberals and conservatives in terms of environmental attitudes.

This is also on track to worsen over time. As one of my interviewees acknowledged, the consequences of climate change will affect natural resources like food crops and water, thus bringing increased competition for natural resources (IPCC, 2014a). Related to this, an increase in migration from low lying coastal areas could increase perceptions of competition for space. With this in mind, climate change has the potential to increase perceptions of the world as a competitive jungle, which is the precursor worldview to SDO (Duckitt, 2001). Endorsement of SDO may also be a way of responding to threat (Jost et al., 2003). Based on these observations, 
there could be bidirectional associations between holding an SDO causally relating to climate change (through greater inaction and environmental exploitation), and the effects of climate change causally related to holding an SDO (through increased perceptions of competition).

I show that the endorsement of SDO is largely incompatible with many aspects of pro-environmentalism, extending beyond a simple denial of climate change. This suggests that high-SDO individuals see the environment in a different way: As a resource to be exploited to enhance our way of life, rather than something to be protected. Individuals who are less concerned about inequality are perhaps instead more concerned about their own interests. This is consistent with Steg and Vlek's (2009, p. 311) observations, that "the more strongly individuals subscribe to values beyond their immediate own interests, that is, self-transcendent, prosocial, altruistic or biospheric values, the more likely they are to engage in proenvironmental behaviour". Duckitt (2001) argues that endorsement of SDO is a direct rejection of self-transcendent values such as universalism and benevolence, and is instead aligned with achievement and power value dimensions (self-enhancement).

Because SDO and RWA are identified as barriers to environmental engagement, these variables (and the syndromes they represent) ought to be taken into consideration when designing climate change communication. Individuals relatively higher in these ideologies can be expected to be more resistant to changing (e.g. as indicated by lower willingness to make sacrifices), and therefore more immune to pleas for help on the environment. They also might perceive action on climate change in a different way to their more liberal counterparts. For instance, high-SDOs are threat-sensitive under some conditions, and thus wary of mitigating action that may be costly or has the potential to disrupt the current hierarchical social system. Ideology is a more difficult barrier to bridge than a simple misunderstanding of the science, and therefore sheds some light on why climate change belief is not more strongly predicted by education level or knowledge about climate change (Hornsey et al., 2016).

My research also has several implications for measurement, both of SDO and environmentalism. I demonstrated that the associations between these variables are relatively consistent, regardless of the measure of SDO employed - including 
shortened scales - and regardless of the aspect of environmentalism measured. This is testament to how robust the SDO-environmentalism relationship is. I also identified that high-SDO participants in my samples (and consistent with those of other researchers) tend not to endorse hierarchy per se, but instead are more tolerant of it than low-SDOs. I discuss this as a limitation below, but also explore here what this means for understanding and conceptualizing SDO as a construct.

A negative association between SDO and environmentalism has previously been taken in the literature (and in my thesis) as meaning that greater endorsement of SDO is related to lower pro-environmentalism. For example, high-SDOs are frequently referred to as individuals who "prefer unequal social systems" (Clarke et al., 2019, p. 1). However, there are typically very few individuals that actually endorse SDO, making this common interpretation somewhat flawed. This is not to say that the preference for inequality is unrelated to environmentalism, but instead that researchers ought to interpret the relationships within the constraints of their dataset. Such a finding might be demonstrating that it is not the 'preference' for inequality per se, but instead the tolerance of inequality that is problematic for the environment.

The ideology-environmentalism association was largely consistent across various indicators of environmentalism, which all tend to be interrelated. This could suggest that elements such as pro-environmental attitudes, behavioural intentions, and belief in the severity of environmental problems (i.e. climate change) might all contribute to measuring a general overarching construct of 'environmentalism'. While I draw parallels between the generality of this environmentalism and the generality of prejudice (as in Chapter 2), there do remain some subtle differences. For instance, RWA more strongly predicts the opposition of Green politics than SDO. Furthermore, there are distinctions in how the two dimensions of SDO relate to various elements of environmentalism, with endorsement of SDO-D relating to the rejection of business and government-level responsibility for acting on climate change, while SDO-E relates more strongly to refusing personal responsibility.

Therefore, while the associations are similar, and hence suggest that endorsing these conservative ideologies relate to a general rejection of pro-environmentalism, differences do exist. I further speculate that the reasons underlying the associations 
likely differ, for example Social Dominants may reject environmental engagement in order to avoid the associated financial costs of action (e.g. Clarke et al., 2019). Future research to thoroughly explore the reasons behind these associations would helpfully inform possible ways of framing environmental engagement that does appeal to those with conservative ideologies.

Given the range of measures available, and potential to find subtle distinctions in their predictions, I still agree with the argument I made in the Introduction (Chapter 1) that multiple indices of environmentalism ought to be included in research in this area. To guide future research, when interested in examining the association with conservative ideologies, it is sensible to include a measure of environmental policy support. This ties into the original purpose of the political polarization of climate change, which served to undermine support for pro-environmental policy (McCright $\&$ Dunlap, 2010). However, some research indicates that increasing policy support may simultaneously undermine environmental activism (Levine \& Kline, 2017), and therefore a separate measure of personal action intentions is also warranted. I also found that many people note that environmental action is characterised by competing interests (Chapter 5), and this is perhaps particularly relevant for conservatives. Specifically, framing environmental action at the expense of the economy lowers support, and this is especially the case for conservatives (Harring \& Sohlberg, 2017). To contribute to the measurement of this, I have introduced a new set of items that might be useful in seeing where individuals across the ideological spectrum place their opinions when faced with trade off decisions.

\section{Strengths and Limitations}

I would like to briefly note some strengths of this work, before acknowledging its limitations. First, my thesis includes a relatively large number of studies, with thousands of participants. My work spans both student and general community samples, noting differences between them, and includes examination of both crosssectional and longitudinal associations between the variables of interest. Where possible, I replicated my findings, rather than relying on single studies to draw conclusions (i.e. Chapters 3, 4, and 6). I also made use of both quantitative and qualitative methods, culminating in a reliable scale derived from actual interviews. 
With this in mind, there are several weaknesses to point out, both as limitations of my work and as potential avenues for future research.

As I have alluded to already, scores on the SDO scale demonstrated restriction of range across the studies in my thesis, in that scale scores are distributed primarily below the theoretical scale midpoint. This is not a limitation for the robustness of statistical tests, as the distribution of scores were neither skewed nor kurtotic, and hence did not violate assumptions of normality. Instead, scores were normally distributed, but participants generally did not make use of the full scale. For example, the individual with the highest level of SDO in Studies 4, 5a and 5b scored on average only just above the midpoint of the scale. It is therefore more appropriate to describe the high-SDO groups as indifferent to inequality, rather than a group characterized by a preference for it. This raises a problem for interpreting the attitudes of 'high' scorers on the SDO scale, as researchers are perhaps mischaracterizing ambivalence as endorsement.

I am not the only researcher to report such a distribution of SDO scores, though I am unaware of other places where this issue has been fully explored. Several researchers (e.g. Altemeyer, 2003, 2004; Overbeck et al., 2004) have used individuals who score in the top quartile to denote a 'high SDO' group. However, to my knowledge, none report the high-SDO group's actual level of endorsement of SDO in relation to the scale. It is therefore unclear if these high-SDO groups are similar to those reported here, meaning high scorers relative to other participants, or if it is a true high-SDO group, meaning high scores relative to the scale.

To examine the possible extent of this issue in this literature, I again returned to the information collected for the meta-analyses. Participants in the studies where means and standard deviations were available $(\mathrm{k}=27)$ for 7 -point scales on average disagreed with the items $\left(M_{\text {mean }}=2.58, M_{\mathrm{SD}}=.94\right)$. Using the average mean and standard deviations from these samples, the majority (68\%) of individuals score between 1.64 and 3.52 on the scale, and almost everyone (95\%) scores between 0.70 (a score that is out of range below the possible minimum score of 1 , therefore is indexed by strong disagreement on all items) and 4.46 on the scale, as calculated by \pm 1 and 2 standard deviations, respectively. This means that only a very small percentage (perhaps no greater than 5\%) of people actually endorse SDO (i.e. score 5, 
6 , or 7 on the 7-point scale, indicating agreement with the items). It is therefore inappropriate to comment on the relationship between SDO and environmentalism beyond these upper limits.

Without true high-SDO scorers (relative to the scale), it is impossible to know how individuals who genuinely prefer inequality and dominance would place themselves in terms of environmental engagement, or whether they exist at all. Higher scores on the SDO scale could continue the linear relationship between these variables, such that the stronger one endorses SDO, the less pro-environmental they are. This would mean that these high scorers are even more opposed to acting on climate change than the midpoint-high SDOs. Alternatively, the linear relationship may not continue. For example, at more extreme scores the variables could instead exhibit a curvilinear association. This could look like diminishing gains, where additional increases in SDO are unrelated to environmentalism scores. Another (unlikely) possibility is a U-shaped curve, such that those scoring at the extreme upper end of SDO may tend back towards pro-environmentalism.

Alternatively, individuals at the extreme end might not actually exist. This is supported from examination of histograms and frequency charts, which reveal (at least in my samples) that there really are so few 'true' Social Dominants. It could also be the case that high-SDOs are around, but uninterested in participating in research. As some evidence for the second point, individuals who were unwilling to be contacted for a future study during the preselection phase in Chapter 5 were significantly higher in SDO than those who were willing. For the actual interview study, I ended up with only 19 of the 20 invited high-SDOs, as my final participant did not show up for their interview.

Assuming they do exist, individuals at the upper end of the SDO scale are a group I have not managed to recruit, and therefore I cannot generalize my findings to them. However, based on the theoretical rationale of why these variables are related, I expect that the linear association would continue. Given that there are few, if any, individuals that actually do endorse high levels of SDO, it is perhaps unimportant to focus research efforts on identifying these people to assess their environmental attitudes. The main takeaway from this limitation is that the SDO scale as used here is not a measure of the extent to which one endorses or opposes intergroup dominance, 
but instead a measure of the tendency towards, versus indifference of, equality. I recommend that in future, researchers use the scale anchors to benchmark where 'high' SDO groups sit to help guide interpretation of results.

The most concerning criticism that stems from this 'restriction of range' is that the groups that I identified as high-SDOs could be individuals who tend to place themselves on the midpoint of all scales. Specifically, these individuals on average neither agree nor disagree with SDO scale items (while the majority of the sample on average disagrees), and similarly score closer to the midpoint on measures of environmentalism (while the majority of the sample leans towards proenvironmentalism). This pattern of responding, where individuals are perhaps systematically choosing the middle of a series of response options, could be a midpoint response bias. Kieruj and Moors (2010) define a midpoint response bias as occurring "when the midpoint or middle response categories are relatively more chosen than the adjacent categories." (p. 328). Participants clearly do not express this bias on average, given that means differ from the midpoint on most SDO and environmental measures. However, it is possible that some participants respond at the midpoint more than others (e.g. Hernádez, Dasgow \& González-Romá, 2004), which could be those I am capturing.

Future work ought to explore this possibility, though there are several reasons to reject the midpoint interpretation of my findings. Perhaps reassuringly, He, Van de Vijver, Espinosa and Mui (2014) found that midpoint responding was unrelated to many variables similar to, or that ought to correlate with, SDO. For example, midpoint responding was unrelated to Schwartz's egalitarianism measure, unrelated to gender egalitarianism, and related negatively to hierarchy values. One finding from my thesis that cannot be explained by a midpoint bias interpretation is the longitudinal results from Study 2B. It is unclear how midpoint responding could explain the decrease in environmentalism over time related to indifference to inequality. In future, seeing whether high-SDOs (midpoint responders) behave differently could give additional evidence that the findings reported in this literature reflect meaningful relationships, as objective ratings of behaviour cannot be subject to a midpoint bias.

In another issue related to this concern, five of the studies in my thesis relied on student samples. This is a problem, given that I demonstrated the association 
between SDO and environmentalism is moderated by sample type. However, because of the temporal, financial, and practical constraints placed on my thesis, I still relied on data from student samples, where the correlation is attenuated. This meant that some weak or null associations that I found when relying on student data may still exist meaningfully in general population samples (e.g. what I found when Study 2a was conceptually replicated in Study 2b). In some ways, this finding is a strength of my research, as it helps to explain some disparate findings. Researchers in this area ought to be aware of the problem, and where possible use general population samples if their aim is to generalize to the wider community.

While there is some evidence for a temporal relationship between variables from the longitudinal research, the majority of the research presented in this thesis is correlational. This is an area that would benefit from some experimental research, to explore whether a causal association exists. Some initial studies are returning promising results. For example, researchers have identified SDO as the mechanism underlying the association between self-transcendent states, such as mindfulness and awe, and environmentalism (Panno et al., 2018; Zhao et al., 2018). Specifically, Panno et al. (2018) demonstrated that more mindful individuals tend to endorse egalitarian intergroup relations (i.e. lower scores on the SDO scale), and this in turn is associated with greater engagement in pro-environmental behaviours.

Providing some experimental evidence of causality, Zhao et al. (2018) succeeded in decreasing endorsement of SDO through an 'awe' manipulation. They found that this decrease in SDO in turn increased environmentalism. The shift in focus to awe, associated with self-transcendence, applied both with awe at the natural environment (e.g. describing the beauty of a sunset, watching an awe-inspiring nature documentary) or a 'social' wonder (childbirth). The authors explained that witnessing such experiences might diminish perceptions of human dominance over nature. This work supports the causal role of SDO in shaping environmental attitudes, and the utility of targeting socially dominant attitudes as a way of boosting proenvironmentalism.

Another relative limitation of my work is that I (as have the majority of other researchers) exclusively captured environmentalism using self-report scales. Furthermore, I largely relied on participant's reported attitudes and intentions, which 
are not perfect indicators of behaviour (e.g. Bamberg \& Möser, 2007). The metaanalyses are somewhat reassuring on this point, as they demonstrate that the SDOenvironmentalism relationship exists across most environment-relevant variables. One possible reason why self-report measures might not accurately capture participants' actual attitudes or behaviour is social desirability bias. If this bias were present, it would mean that participants choose response options that they believe will show them in a favourable light. However, there is doubt as to the extent that this bias affects environmental measures (e.g. Félonneau \& Becker, 2008; Milfont, 2009), and I did not find much evidence for associations with environmentalism items and the social desirability measure included in Chapter 6.

It is also possible that the environmental items and issues conveyed in these items have a different meaning for different participants. Ideally, participants all have a shared understanding of what is being assessed in each item, so that variance in responses captures real individual differences in opinion, rather than differences in comprehension. One example where this might not be the case is for climate change solutions. Perhaps high-SDOs think that acting on climate change involves a different set of actions than those that come to mind for low-SDOs, and therefore respond differently. This provides an alternative explanation for why high-SDOs think that climate change action will be relatively more costly than inaction compared to lowSDOs, as some solutions are more expensive.

This interpretation is consistent with political discourses, where for some people 'climate change' is interpreted based on whether the current weather is or is not different. For example, Trump's (2014) tweet: “Snowing in Texas and Louisiana, record setting freezing temperatures throughout the country and beyond. Global warming is an expensive hoax!", conflating and confusing climate change and global warming, and weather with climate. Meanwhile, others note changes in climate as long-term variability in the average weather of a region, consistent with the IPCC (2014a) and other scientific analyses of climate change.

Finally, it is important to note that while SDO and RWA exhibit relatively consistent and significant associations with environment-relevant variables, they do not fully explain attitudes towards the environment and climate change. Instead, the effect sizes are relatively small, explaining part of the variance in these attitudes. 
Putting the effect sizes in context, even relatively small changes in behaviour correspond to significant reductions in greenhouse gas emissions (e.g. Beckage et al., 2018; Heller, Willits-Smith, Meyer, Keoleian, \& Rose, 2018; Wynes \& Nicholas, 2017). Therefore, research on small influences on environmental engagement is still important. More research is needed to clarify all influences on environmental attitudes, and work on removing barriers to action.

\section{Future research}

While the research in this thesis clarifies some aspects of how SDO and environmentalism relate, I did not (as I had hoped) have the space to explore interventions that might motivate high-SDOs to act. One sensible next step in this area is to work on developing, and testing, ways of framing climate change that might appeal to relative high-SDOs. It is unlikely that they will respond in the same way to climate change communications as other groups, and therefore messages should be tailored (i.e. segmentation, Feinberg \& Willer, 2013). Some studies show that the way climate change is framed influences participant's willingness to engage with the issue, though the same message elicits different responses from conservatives and liberals. For example, denial is higher among conservatives when the issue is referred to as 'global warming' rather than 'climate change', while the wording makes no difference for liberals (Schuldt, Konrath \& Schwarz, 2011).

Other research has demonstrated some success framing environmental action in a way that conservatives (who are typically higher in SDO) relate to. For instance, they are more responsive to such appeals when they come from other conservatives (e.g. Wolsko, Ariceaga \& Seiden, 2016) or religious leaders (Maibach et al., 2015; Schuldt, Pearson, Romero-Canyas \& Larson-Konar, 2016). Messages are also more convincing when they articulate and align with conservative values, such as appealing to purity concerns, to obeying authority, or framing environmental actions as acts of patriotism (Feinberg \& Willer, 2013; Wolsko et al., 2016). Finally, the partisan gap in climate change engagement is virtually eradicated when conservatives are asked to compare the present with the past, as opposed to the future (Baldwin \& Lammers, 2016). 
While the research outlined above suggests that framing effects have the potential to increase conservative's environmental engagement, other research suggests that conservatives are somewhat immune to many climate change frames (e.g. Feldman \& Hart, 2018), and they also neglect to consider the role of SDO specifically. This highlights the need for further research into when and how frames resonate with conservatives, and those with relatively higher SDO, in order to identify effective strategies to communicate climate change in a way that bridges the ideological divide. This is an area of research that $\mathrm{I}$ hope to transition into in the coming years. This work would need to be designed with care, as much of the framing research has found that those already dismissive of climate change may become less pro-environmental after certain manipulations, such as those highlighting the national security (Myers, Nisbet, Maibach \& Leiserowitz, 2012), economic (Harring \& Sohlberg, 2017), justice (Whitmarsh \& Corner, 2017), or health (Hart \& Nisbet, 2012) implications of climate change.

Future research into experimentally manipulating SDO levels, by undermining support for inequality and hierarchy, would also be beneficial. For instance, using an awe manipulation, Zhao et al. (2018) showed that SDO levels declined. It would be useful to test the limits of awe interventions, for instance assessing effects on behaviour as well as attitudes, and whether these are sustained over time. Based on Duckitt's (2001) observations that a competitive worldview leads to SDO, future research could test manipulations that enhance or reduce perceptions of competition. These should, in turn, lead to increases or decreases in SDO, and subsequent changes in environmental attitudes. As well as having a positive influence on environmental attitudes, manipulations that successfully reduce SDO levels may also improve intergroup relations and other prejudicial correlates of SDO.

Given that the research presented here drew from New Zealand samples, research should continue to examine the ideology-environmentalism link overseas. New Zealand is a more strongly ideologised country (Duckitt, 2001), as evidenced by the relatively stronger link between SDO and RWA. New Zealanders are also socialized more strongly into authoritarianism than inequality and dominance, hence why RWA is related to increases in SDO over time, but not the reverse (Study 2B). Based on this, it is possible that the relationship between these ideologies and 
environmentalism could differ across countries, given that the ideologies likely relate differently. Future research could therefore explore how SDO and RWA relate to environmental attitudes in settings where climate change is more politically polarized, such as the United States, which Duckitt (2001) claims is less ideologically organized than NZ and Western European countries.

Research could also continue clarifying the role of the two facets of SDO in relation to environmentalism, and with a greater variety of environment-relevant variables. With enough research in this area, the associations could be meta-analysed to get a better idea of how and when SDO-E or SDO-D is the stronger correlate. Furthermore, given that RWA is a predictor of similar strength, it would also be useful to broaden the three-dimensional RWA approach to the environmental sphere (e.g. as in Clarke et al., 2019; Reese, 2012). To my knowledge, no research to date has constructed Duckitt's (2001) dual-process motivational model with worldviews predicting each facet of SDO and RWA, in turn relating to environmentalism. This could be an important next step, as it is possible that the dimensions of SDO are not uniformly predicted by perceptions of competition, and similarly for the dimensions of RWA (aggression, conservatism, and traditionalism; Duckitt et al., 2010) predicted by perceptions of danger.

There are also interesting avenues for future research examining the ideologyenvironmentalism link qualitatively. Continued interview-based work in this area would be helpful for generating knowledge on more exploratory questions, such as: Do high-SDOs (or laypeople in general) recognise climate change as a social justice issue? Do they understand the impact of their behaviours, not just on the environment, but the likely consequences for low-status groups? While researchers are increasingly concerned about these justice dimensions of climate change (e.g. Jylhä, 2016), it is unclear whether this has entered the public conscience. Probing these questions with qualitative research could help determine how laypersons view the sociocultural consequences of climate change, which I did not capture in my interview study, or perhaps how individuals react when faced with such facts. While the information gleaned from such future work may help generate new theories, this would need to be followed up by quantitative research in order to fully test such theories. 


\section{Conclusions}

Social dominance theory (SDT; Sidanius \& Pratto, 1999) provides a framework for understanding how societies are organized in group-based hierarchies. SDT also explains how this hierarchy is maintained through a network of legitimizing myths, which help individuals justify the unequal distribution of resources and social value to groups at the top of the hierarchy, and away from groups at the bottom. My thesis supports the growing pool of research extending SDT to aid understanding of how individuals use the environment in hierarchical and unequal ways. Those who are relatively more tolerant of social inequality are also happier to continue exploiting the environment, and more resistant to making sacrifices for the environment.

I set out to gain a better understanding of individual differences in attitudes towards social and enironmental issues. I have made some progress towards this, though note that we still have far to go. I sincerely hope that researchers continue to clarify the role of ideology in environmental attitudes. I also wish to highlight the need to develop interventions that might spur even those tolerant of inequality to action. 


\section{References}

Abrahamse, W., \& Steg, L. (2013). Social influence approaches to encourage resource conservation: A meta-analysis. Global Environmental Change, 23, 1773-1785.

Adorno, T. W., Frenkel-Brunswik, E., Levinson, D. J., \& Sanford, R. N. (1950). The Authoritarian Ppersonality. New York: Harper.

Agyeman, J., Schlosberg, D., Craven, L., \& Matthews, C. (2016). Environmental Justice: From inequity to everyday life, community, and just sustainabilities. Annual Review of Environment and Resources, 41, 321-340.

Ahn, S., Ames, A. J., \& Myers, N. D. (2012). A review of meta-analyses in education: Methodological strengths and weaknesses. Review of Educational Research, 82, 436-476.

Aitken, C., Chapman, R., \& McClure, J. (2011). Climate change, powerlessness and the commons dilemma; Assessing New Zealander's preparedness to act. Global Environmental Change, $21,752-760$.

Ajzen, I. 1991. The theory of planned behavior. Organizational Behavior and Human Decision Processes, 50, 179-211.

Allison, S., Woodall, T., Armannsdottir, G., \& Pich, C. (2017, July). It's just for old men and children: exploring self-image barriers to cycling. Paper presented at the 50th Academy of Marketing Conference, Hull University Business School, England.

Aloe, A. M. (2009). A partial effect size for the synthesis of multiple regression models. (Doctoral dissertation). Florida State University.

Altemeyer, B. (1981). Right-wing authoritarianism. Winnipeg, Canada: University of Manitoba Press.

Altemeyer, B. (1998). The other "authoritarian personality". Advances in Experimental Social Psychology, 30, 47-92.

Altemeyer, B. (1996). The Authoritarian Specter. Cambridge, MA: Harvard University Press.

Altemeyer, B. (2003). What happens when authoritarians inherit the earth: A simulation. Analyses of Social Issues and Public Policy, 3, 161-169.

Altemeyer, B. (2004). Highly dominating, highly authoritarian personalities. Journal of Social Psychology, 144, 421-447. 
Althor, G., Watson, J., \& Fuller, R. (2016). The inequity in climate change. Australasian Science, 37, $1-6$.

Amérigo, M., Palavecinos, M., García, J. A., Román, F., \& Trizano-Hermosilla, I. (2017). Effects of the social dominance orientation on environmental attitudes of Chilean university students. International Journal of Social Psychology, 32, 136-163.

Anable, J., Lane, B., \& Kelay, T. (2006). An evidence base review of public attitudes to climate change and transport behaviour. The Department for Transport report: United Kingdom.

Archie, K. M., Chapman, R., \& Flood, S. (2018). Climate change response in New Zealand communiites: Local scale adaptation and mitigation planning. Environmental Development, 28, 19-31.

Ard, K. (2015). Trends in exposure to industrial air toxins for different racial and socioeconomic groups: A spatial and temporal examination of environmental inequality in the U.S. from 1995 to 2004. Social Science Research, 53, 375-390.

Asbrock, F., Sibley, C. G., \& Duckitt, J. (2010). Right-wing authoritarianism and social dominance orientation and the dimensions of generalized prejudice: A longitudinal test. European Journal of Personality, 24, 324-340.

Austin, D. E., \& Jackson, M. (2019). Benevolent and hostile sexism differentially predicted by facets of right-wing authoritarianism and social dominance orientation. Personality and Individual Differences, 139, 34-38.

Bachman, R., \& Schutt, R. K. (2013). The practice of research in criminology and criminal justice. United States of America: Sage Publications.

Bailey, I. G., \& Jackson Inderberg, T. H. (2016). New Zealand and climate change: what are the stakes and what can New Zealand do? Policy Quarterly, 12, 3-12.

Baldwin, M., \& Lammers, J. (2016). Past-focused environmental comparisons promote proenvironmental outcomes for conservatives. PNAS, 113, 14953-14957.

Bamberg, S., \& Möser, G. (2007). Twenty years after Hines, Hungerford, and Tomera: A new metaanalysis of psycho-social determinants of pro-environmental behaviour. Journal of Environmental Psychology, 27, 14-25. 
Beckage, B., Gross, L., Lacasse, K., Carr, E., Metcalf, S., Winter, J., . . \& \& Hoffman, F. (2018). Linking models of human behaviour and climate alters projected climate change. Nature Climate Change, 8, 79-84.

Becker, B. J., \& Wu, M. J. (2007). The synthesis of regression slopes in meta-analysis. Statistical science, 22, 414-429.

Benegal, S. D. (2018). The spillover of race and racial attitudes into public opinion about climate change. Environmental Politics, 27, 1-24.

Ben-Nun, P. (2008). Respondent Fatigue, in P. J. Lavrakas Encyclopedia of survey research methods. United States of America: Sage Publications.

Bergh, R., Sidanius, J., \& Sibley, C. G. (2015). Dimensions of social dominance: Their personality and socio-political correlates within a New Zealand probability sample. New Zealand Journal of Psychology, 44, 25-34.

Biggerstaff, D. (2012). Qualitative research methods in psychology. In Psychology - selected papers, Dr Gina Rossi (Ed), 175-206.

Boden, T. A., Marland, G., \& Andres, R. J. (2017). Global, Regional, and National Fossil-Fuel CO2 Emissions. Carbon Dioxide Information Analysis Center, Oak Ridge National Laboratory, U.S. Department of Energy, Oak Ridge: United States of America.

Bollen, K. A. (2002). Latent variables in psychology and the social sciences. Annual Review Psychology, 53, 605-634.

Borenstein, M., Hedges, L. V., Higgins, J. P. T., \& Rothstein, H. R. (2009). Introduction to metaanalysis. John Wiley \& Sons, Ltd.

Brandt, M. J., \& Henry, P. J. (2012). Gender inequality and gender differences in authoritarianism. Personality and Social Psychology Bulletin, 38, 1301-1315.

Braun, V., \& Clarke, V. (2006) Using thematic analysis in psychology. Qualitative Research in Psychology, 3, 77-101

Braun, V., \& Clarke, V. (2013). Successful qualitative research: A practical guide for beginners. United Kingdom: Sage Publications.

Briggs, S., \& Cheek, J. (1986). The role of factor analysis in the development and evaluation of personality scales. Journal of Personality, 54, 106-148. 
Brody, S., Grover, H., \& Vedlitz, A. (2012). Examining the willingness of Americans to alter behavior to mitigate climate change. Climate Policy, 12, 1-22.

Brossard, D., Shanahan, J. \& McComas, K. (2004) Are issue-cycles culturally constructed? A comparison of french and american coverage of global climate change. Mass Communication and Society, 7, 359-377.

Brown, T. A. (2014). Confirmatory factor analysis for applied research. United States of America: Guilford Publications.

Bryant, B. (1995). Environmental justice: Issues, policies, and solutions. United States of America: Island Press.

Burgess, J., Harrison, C., Filius, P. (1998). Environmental communication and the cultural politics of environmental citizenship. Environment and Planning, 30, 1445-1460.

Caddick, Z. A. (2016). Evaluating contradicting and confirming evidence: A study on beliefs and motivated reasoning (Master's thesis). San José State University, California, United States.

Campbell, D. T. (1063). From description to experimentation: Interpreting trends as quasiexperiments. In C. W. Harris (eds.), Problems in measuring change. Madison: University of Wisconsin Press.

Campbell, D. T., \& McCandless, B. R. (1951). Ethnocentrism, xenophobia, and personality. Human Relations, 4, 186-192.

Cantal, C., Milfont, T. L., Wilson, M. S., \& Gouveia, V. V. (2015). Differential effects of right-wing authoritarianism and social dominance orientation on dimensions of generalized prejudice in Brazil. European Journal of Personality, 29, 17-27.

Carlton, J. S., Perry-Hill, R., Huber, M., \& Prokopy, L. S. (2015). The climate change consensus extends beyond climate scientists. Environmental Research Letters, 10, 1-12.

Carmines, E., \& McIver, J. (1981). Analyzing Models With Unobservable Variables: Analysis of Covariance Structures, in Social Measurement: Current Issues (pp. 65-115). United States of America: Sage Publications.

Casey, G., \& Galor, O. (2017). Is faster economic growth compatible with reductions in carbon emissions? The role of diminished population growth. Environmental Research Letters, 12, $1-8$. 
Chen, C., Noble, I., Hellmann, J., Coffee, J., Murillo, M., \& Chawla, N. (2015). University of Notre Dame Global Adaptation Index Country Index Technical Report. ND-GAIN: South Bend, IN, USA.

Chen, C. F., Xu, X., \& Day, J. K. (2017). Thermal comfort or money saving? Exploring intentions to conserve energy among low-income households in the United States. Energy Research \& Social Science, 26, 61-71.

Chiles, R. M., Fabian, E. E., Tobin, D., Colby, S. J., \& DePue, S. M. (2018). Greenhouse gas emissions from agriculture: Reconciling the epistemological, ethical, political, and practical challenges. Journal of Agricultural and Environmental Ethics, 31, 341-348.

Cialdini, R. B. (2005). Basic social influence is underestimated. Psychological inquiry, 16, 158-161.

Clark, H., Kelliher, F., \& Pinares-Patino, C. (2011). Reducing CH4 Emissions from grazing ruminants in New Zealand: Challenges and opportunities. Asian-Australian Journal of Animal Science, 24, 295-302.

Clarke, E. J. R., Ling, M., Kothe, E. J., Klas, A., Richardson, B. (2019). Mitigation system threat partially mediates the effects of right-wing ideologies on climate change beliefs. Journal of Applied Social Psychology, 00, 1-12.

Climate Change Adaptation Technical Working Group. (2017). Adapting to climate change in New Zealand. ISBN: 978-1-98-852527-3

Cohen, J. (1988). Statistical power analysis for the behavioral sciences (2nd ed.). United States of America: Erlbaum.

Collins, M., Knutti, R., Arblaster, J., Dufresne, J-L., Fichefet, T., Friedlingstein, P., ... Wehner, M. (2013). Long-term Climate Change: Projections, Commitments and Irreversibility. In: Climate Change 2013: The Physical Science Basis. Contribution of Working Group I to the Fifth Assessment Report of the Intergovernmental Panel on Climate Change [Stocker, T.F., D. Qin, G.-K. Plattner, M. Tignor, S.K. Allen, J. Boschung, A. Nauels, Y. Xia, V. Bex and P.M. Midgley (eds.)]. Cambridge University Press, Cambridge, United Kingdom and New York, NY, USA.

Comparative Agendas Project. (2016). Gallup's most important problem. (Unpublished raw data). Comrey, A. L., \& Lee, H. B. (1992). Interpretation and application of factor analytic results. In A. L. Comrey \& H. B. Lee: A first course in factor analysis. 
Cook, J., Oreskes, N., Doran, P. T., Anderegg, W. R., Verheggen, B., Maibach, E. W., ... \&

Nuccitelli, D. (2016). Consensus on consensus: a synthesis of consensus estimates on humancaused global warming. Environmental Research Letters, 11, 1-7.

Cooper, H. (1998). Synthesizing research: A guide for literature reviews (3rd ed.). United States: Sage Publications.

Cordano, M., Welcomer, S. A., \& Scherer, R. F. (2003). An analysis of the predictive validity of the new ecological paradigm scale. Journal of Environmental Education, 34, 22-28.

Coyle, F., Fairweather, J. (2005). Challenging a place myth: New Zealand's clean green image meets the biotechnology revolution. Area, 37, 148-158.

Cumming, G. (2013). Understanding the new statistics: Effect sizes, confidence intervals, and metaanalysis. United States of America: Routledge.

Cushing, L., Morello-Frosch, R., Wander, M., \& Pastor, M. (2015). The haves, the have-nots, and the health of everyone: the relationship between social inequality and environmental quality. Annual Review of Public Health, 36, 193-209.

Dawes, R. M., \& Messick, D. M. (2000). Social dilemmas. International Journal of Psychology, 35, 111-116.

de Sousa-Neto, E. R., Gomes, L., Nascimento, N., Pacheco, F., \& Ometto, J. P. (2018). Land use and land cover transition in Brazil and their effects on greenhouse gas emissions. (pp. 309-

321).In M. A. Muñoz \& R. Zornoza. Soil Management and Climate Change, Academic Press.

Delicado, A. (2012). Environmental education technologies in a social void: The case of

'Greendrive'. Environmental Education Research., 18, 831.

Devine-Wright, P., Price, J., \& Leviston, Z. (2015). My country or my planet? Exploring the influence of multiple place attachments and ideological beliefs upon climate change attitudes and opinions. Global Environmental Change, 30, 68-79.

Diamantopoulos, A., Sarstedt, M., Fuchs, C., Wilczynski, P., \& Kaiser, S. (2012). Guidelines for choosing between multi-item and single-item scales for construct measurement: A predictive validity perspective. Journal of the Academy of Marketing Science, 40, 434-449.

DiMaggio, P., Evans, J. H., \& Bryson, B. (1996). Have Americans' Social Attitudes Become More Polarized? American Journal of Sociology, 102, 690-755. 
Dimdins, G., Sandgren, M., \& Montgomery, H. (2016). Psychological variables underlying political orientations in an old and a new democracy: A comparative study between Sweden and Latvia. Scandinavian Journal of Psychology, 57, 437-445.

Doran, P. T., \& Zimmerman, M. K. (2009). Examining the scientific consensus on climate change. Transactions American Geophysical Union, 90, 22-23.

Downs, A. (1972). Up and Down with Ecology-The 'Issue-Attention Cycle.' The Public Interest, 28, 38-50.

Duckitt, J. (2001). A dual-process cognitive-motivational theory of ideology and prejudice. In: Advances in experimental social psychology (Vol. 33, pp. 41-113). Academic Press.

Duckitt, J., Bizumic, B., Krauss, S. W., \& Heled, E. (2010). A tripartite approach to right-wing authoritarianism: The authoritarianism-conservatism-traditionalism model. Political Psychology, 31, 685-715.

Duckitt, J., \& Sibley, C. G. (2007). Right wing authoritarianism, social dominance orientation and the dimensions of generalized prejudice. European Journal of Personality, 21, 113-130.

Duckitt, J., \& Sibley, C. G. (2010). Personality, ideology, prejudice, and politics: A dual-process motivational model. Journal of personality, 78, 1861-1894.

Duckitt, J., Wagner, C., du Plessis, I., \& Birum, I. (2002). The psychological bases of ideology and prejudice. Testing a dual process model. Journal of Personality and Social Psychology, 83, 75-93.

Dunlap, R. E. (2008). The new environmental paradigm scale: From marginality to worldwide use. The Journal of Environmental Education, 40, 3-18.

Dunlap, R. E. \& Van Liere, K. D. (1978). The new environmental paradigm: A proposed measuring instrument and preliminary results. Journal of Environmental Education, 9, 10-19.

Duval, S., \& Tweedie, R. (2000). Trim and fill: A simple funnel-plot-based method of testing and adjusting for publication bias in meta-analysis. Biometrics, 56, 455-463.

Eagly, A. H., Diekman, A. B., Johannesen-Schmidt, M. C., \& Koenig, A. G. (2004). Gender gaps in sociopolitical attitudes: A social psychological analysis. Journal of Personality and Social Psychology, 87, 796-816.

Egan, P. J. \& Mullin, M. (2017). Climate change: US public opinion. Annual Reviews, 20, 209-227. 
Ekholm, S., \& Olofsson, A. (2017). Parenthood and worrying about climate change: the limitations of previous approaches. Risk Analysis, 37, 305-314.

Erez, A., Bloom, M. C., \& Wells, M. T. (1996). Using random rather than fixed effects models in meta-analysis: Implications for situational specificity and validity generalization. Personnel Psychology, 49, 275-306.

Everett, J. A., Caviola, L., Savulescu, J., \& Faber, N. (2018). Speciesism, generalized prejudice and perceptions of prejudiced others. Group Processes and Intergroup Relations.

Fagan, M. (2017). Who's afraid of the ecological apocalypse? Climate change and the production of the ethical subject. The British Journal of Politics and International Relations, 19, 225-244.

Feinberg, M., \& Willer, R. (2013). The moral roots of environmental attitudes. Psychological Science, 24, 56-62

Feldman, L. \& Hart, P. S. (2018). Broadening exposure to climate change news? How framing and political orientation interact to influence selective exposure. Journal of Communication, 68, 503-524.

Félonneau, M. L., \& Becker, M. (2008). Pro-environmental attitudes and behavior: Revealing perceived social desirability. International Review of Social Psychology, 21, 25-53.

Festinger, L. (1957). A theory of cognitive dissonance. United States of America: Stanford university press.

Feygina, I. (2013). Social justice and the human-environment relationship: Common systemic, ideological, and psychological roots and processes. Social Justice Research, 26, 363-381.

Fischer-Kowalski, M., Krausmann, F., \& Pallua, I. (2014). A sociometabolic reading of the Anthropocene: Modes of subsistence, population size and human impact on Earth. The Anthropocene Review, 1, 8-33.

Fitzharris, B. (2007). How vulnerable is New Zealand to the impacts of climate change? New Zealand Geographer, 63, 160-168.

Flouri, E. (2004). Mothers' nonauthoritarian chlid-rearing attitudes in early childhood and children's adult values. European Psychologist, 9, 154-162.

Foley, S. F., Gronenborn, D., Andreae, M. O., Kadereit, J. W., Esper, J., Scholz, D., ... \& Vött, A. (2013). The Palaeoanthropocene: The beginnings of anthropogenic environmental change. Anthropocene, 3, 83-88. 
Gardner, B., \& Abraham, C. (2008). Psychological correlates of car use: A metaanalysis. Transportation Research Part F: Traffic Psychology and Behaviour, 11, 300-311.

Gardner, G. T., \& Stern, P. C. (1996). Environmental problems and human behavior. United States of America: Allyn \& Bacon.

Gifford, R. (2011). The dragons of inaction: psychological barriers that limit climate change mitigation and adaptation. American psychologist, 64, 290.

Global footprint network. (2018). Earth overshoot day. Online resource: https://www.overshootday.org/newsroom/past-earth-overshoot-days/

Gneezy, U., Meier, S., \& Rey-Biel, P. (2011). When and why incentives (don't) work to modify behavior. Journal of Economic Perspectives, 25, 191-210.

Guillebaud, J., \& Hayes, P. (2008). Population growth and climate change. British Medical Journal, $337,1-2$.

Gunn, L. D., Greenham, B., Davern, M., Mavoa, S., Taylor, E. J., \& Bannister, M. (2017). Environmental justice in Australia: Measuring the relationship between industrial odour exposure and community disadvantage. In Community quality-of-life indicators: Best cases VII (pp. 113-133). Springer, Cham.

Hadarics, M., \& Kende, A. (2018). The dimensions of generalized prejudice within the dual-process model: The mediating role of moral foundations. Current Psychology, 37, 731-739.

Häkkinen, K., \& Akrami, N. (2014). Ideology and climate change denial. Personality and Individual Differences, 70, 62-65.

Hall, M. P., Lewis, N. A., \& Ellsworth, P. C. (2018). Believing in climate change, but not behaving sustainably: Evidence from a one-year longitudinal study. Journal of Environmental Psychology, 56, 55-62.

Hamilton, L. C. (2011). Education, politics and opinions about climate change evidence for interaction effects. Climatic Change, 104, 231-242.

Harman, H. H. (1960). Modern Factor Analysis. United States of America: University of Chicago Press.

Harring, N., \& Sohlberg, J. (2017). The varying effects of left-right ideology on support for the environment: Evidence from a Swedish survey experiment. Environmental Politics, 26, 278300. 
Harrison, F. (2011), Getting started with meta-analysis. Methods in Ecology and Evolution, 2, 1-10.

Hatemi, P. K., \& McDermott, R. (2016). Give me attitudes. Annual Review of Political Science, 19, 331-350.

Hawcroft, L. J., \& Milfont, T. L. (2010). The use (and abuse) of the new environmental paradigm scale over the last 30 years: A meta-analysis. Journal of Environmental Psychology, 30, 143158.

He, J., Van de Vijver, F. J., Espinosa, A. D., \& Mui, P. H. (2014). Toward a unification of acquiescent, extreme, and midpoint response styles: A multilevel study. International Journal of Cross Cultural Management, 14, 306-322.

Heller, M. C., Willits-Smith, A., Meyer, R., Keoleian, G. A., \& Rose, D. (2018). Greenhouse gas emissions and energy use associated with production of individual self-selected US diets. Environmental Research Letters, 13, 1-11.

Helmus, M. (2017). Personal communication - advice on meta-analytic methods.

Hernández, A., Drasgow, F., \& González-Romá, V. (2004). Investigating the functioning of a middle category by means of a mixed-measurement model. Journal of Applied Psychology, 89, 687699.

Higgins, J. P. T., \& Thompson, S. G. (2002). Quantifying heterogeneity in a meta-analysis. Statistics in Medicine, 21, 1539-1558.

Hines, J. M., Hungerford, H. R., \& Tomera, A. N. (1987). Analysis and synthesis of research on responsible environmental behaviour: A meta-analysis. Journal of Environmental Education, $18,1-8$.

Hites, R. W., \& Kellog, E. P. (1964). The F and Social Maturity Scales in relation to racial attitudes in a deep south sample. Journal of Social Psychology, 62, 189-195.

Ho, A. K., Sidanius, J., Pratto, F., Levin, S., Thomsen, L., Kteily, N., \& Sheehy-Skeffington, J. (2012). Social dominance orientation: Revisiting the structure and function of a variable predicting social and political attitudes. Personality and Social Psychology Bulletin, 38, 583606.

Ho, A. K., Sidanius, J., Kteily, N., Sheehy-Skeffington, J, Pratto, F., Henkel, K. E., Foels, R., \& Stewart, A. L. (2015). The nature of social dominance orientation: Theorizing and measuring 
preferences for intergroup inequality using the new $\mathrm{SDO}_{7}$ scale. Journal of Personality and Social Psychology, 109, 1005-1028.

Hoffarth, M. R., \& Hodson, G. (2016). Green on the outside, red on the inside: Perceived environmentalist threat as a factor explaining political polarization of climate change. Journal of Environmental Psychology, 45, 40-49.

Hornsey, M. J., Harris, E. A., Bain, P. G., \& Fielding, K. S. (2016). Meta-analyses of the determinants and outcomes of belief in climate change. Nature Climate Change, 6, 622.

Hsiang, S., Kopp, R., Jina, A., Rising, J., Delgado, M., Mohan, S., ... \& Larsen, K. (2017). Estimating economic damage from climate change in the United States. Science, 356, 13621369.

Hu, L. \& Bentler, P. M. (1999) Cutoff criteria for fit indexes in covariance structure analysis: Conventional criteria versus new alternatives, Structural Equation Modeling: A Multidisciplinary Journal, 6, 1-55

Huedo-Medina, T., Sanchez-Meca, J., Marin-Martinez, F., \& Botella, J. (2006). Assessing heterogeneity in meta-analysis: Q statistic or I2 index? CHIP Documents, 19, 1-37.

Hunter, J. E., \& Schmidt, F. L. (1991). Meta-analysis. In Advances in educational and psychological testing: Theory and applications (pp. 157-183). Springer, Dordrecht.

IBM. (2017). SPSS version 23.

IPCC. (2014a). Climate Change 2014: Synthesis Report. Contribution of Working Groups I, II and III to the Fifth Assessment Report of the Intergovernmental Panel on Climate Change [Core Writing Team, R.K. Pachauri and L.A. Meyer (eds.)]. IPCC, Geneva, Switzerland, 151 pp.

IPCC. (2014b). Climate Change 2014: Impacts, Adaptation, and Vulnerability. Part A: Global and Sectoral Aspects. Contribution of Working Group II to the Fifth Assessment Report of the Intergovernmental Panel on Climate Change Mach, M.D. Mastrandrea, T.E. Bilir, sicepiM. Chatterjee, K.L. Ebi, Y.O. Estrada, R.C. Genova, B. Girma, E.S. Kissel, A.N. Levy, S. MacCracken, P.R. Mastrandrea, and L.L. White (eds.)]. Cambridge University Press, Cambridge, United Kingdom and New York, NY, USA, 1132 pp.

Jackson, L. M., Bitacola, L. M., Janes, L. M., \& Esses, V. M. (2013). Intergroup ideology and environmental inequality. Analyses of Social Issues and Public Policy, 13, 327-346. 
Jacques, P. J., Dunlap, R. E., \& Freeman, M. (2008). The Organization of Denial: Conservative Think Tanks and Environmental Skepticism. Environmental Politics, 17, 349-85.

Jetten, J., \& Iyer, A. (2010). Different meanings of the social dominance orientation concept: Predicting political attitudes over time. British Journal of Social Psychology, 49, 385-404.

Jia, F., Alisat, S., Soucie, K., \& Pratt, M. (2015). Generative concern and environmentalism: a mixed methods longitudinal study of emerging and young adults. Emerging Adulthood 3, 306-319.

Jones, C., Hine, D. W., \& Marks, A. D. (2017). The future is now: Reducing psychological distance to increase public engagement with climate change. Risk Analysis, 37, 331-341.

Jo reskog, K. G, So r̈bom, D. (1979). Advances in Factor Analysis and Structural Equation Models. Cambridge: Abt Books

Jost, J. T., Glaser, J., Kruglanski, A. W., \& Sulloway, F. J. (2003). Political conservatism as motivated social cognition. Psychological Bulletin, 129, 339-375.

Jost, J. T., \& Thompson, E. P. (2000). Group-based dominance and opposition to equality as independent predictors of self-esteem, ethnocentrism, and social policy attitudes among African Americans and European Americans. Journal of Experimental Social Psychology, $36,209-232$.

Jylhä, K. M. (2016). Ideological roots of climate change denial. Resistance to change, acceptance of inequality, or both? [Doctoral dissertation]. Sweden: Uppsala University.

Jylhä, K. M., Akrami, N. (2015). Social dominance orientation and climate change denial: The role of dominance and system justification. Personality and Individual Differences, 86, 108-111.

Jylhä, K. M., Cantal, C., Akrami, N., Milfont, T. L. (2016). Denial of anthropogenic climate change: Social dominance orientation helps explain the conservative male effect in Brazil and Sweden Personality and Individual Differences, 98, 184-187.

Kaefer, F. (2014). Credibility at stake? News representations and discursive constructions of national environmental reputation and place brand image: The case of clean, green New Zealand [Doctoral dissertation]. New Zealand: University of Waikato.

Kieruj, N. D., \& Moors, G. (2010). Variations in response style behavior by response scale format in attitude research. International Journal of Public Opinion Research, 22, 320-342. 
Kellstedt, P. M., Zahran, S. \& Vedlitz, A. (2008). Personal efficacy, the information environment, and attitudes toward global warming and climate change in the United States. Risk Analysis, 28, 113-126.

Kempton, W., Boster, J.S. \& Hartley, J.A. (1995). Environmental Values in American Culture. Cambridge: MIT Press.

Kenix, L. J. (2008) Framing science: Climate change in the mainstream and alternative news of New Zealand. Political Science, 60, 117-132.

Kenny, D. A. (2015). Measuring model fit. Website http://davidakenny.net/cm/fit.htm accessed $29 / 08 / 17$

Kenny, D. A., \& Harackiewicz, J. M. (1979). Cross-lagged panel correlation: Practice and promise. Journal of Applied Psychology, 64, 372-379.

Kim, R. S. (2011). Standardized regression coefficients as indices of effect sizes in meta-analysis [Doctoral dissertation]. United States of America: Florida State University.

Kim, J. O., \& Mueller, C. W. (1976). Standardized and unstandardized coefficients in causal analysis: An expository note. Sociological Methods \& Research, 4, 423-438.

Klinsky, S., Roberts, T., Huq, S., Okereke, C., Newell, P., Dauvergne, P., ... \& Bauer, S. (2017). Why equity is fundamental in climate change policy research. Global Environmental Change, 44, 170-173

Klöckner, C. A. (2013). A comprehensive model of the psychology of environmental behaviour: A meta-analysis. Global Environmental Change, 23, 1028-1038.

Kollmuss, A., \& Agyeman, J. (2002). Mind the gap: Why do people act environmentally and what are the barriers to pro-environmental behavior? Environmental Education Research, 8, 239260

Krosnick, J. A., \& Alwin, D. F. (1988). A test of the form-resistant correlation hypothesis: Ratings, rankings, and the measurement of values. Public Opinion Quarterly, 52, 526-538.

Kteily, N., Ho, A. K., \& Sidanius, J. (2012). Hierarchy in the mind: The predictive power of social dominance orientation across social contexts and domains. Journal of Experimental Social Psychology, 48, 543-549. 
Kurz, T., Donaghue, N., Rapley, M., \& Walker, I. (2005). The ways that people talk about natural resources: Discursive strategies as barriers to environmentally sustainable practices. British Journal of Social Psychology, 44, 603-620.

Lacroix, K., \& Gifford, R. (2017). Psychological barriers to energy conservation behaviour: The role of worldviews and climate change risk perception. Environment and Behaviour, 1-32.

Landis, J. R., \& Koch, G. G. (1977). The measurement of observer agreement for categorical data. Biometrics, 33, 159-174.

Laverty, A. A., Webb, E., Vamos, E. P., \& Millett, C. (2018). Associations of increases in public transport use with physical activity and adiposity in older adults. International Journal of Behavioral Nutrition and Physical Activity, 15, 1-10.

Layman, G. C., Carsey, T. M., \& Horowitz, J. (2006). Party Polarization in American Politics. Annual Review of Political Science 9, 83-110.

Lee, I. C., Pratto, F., \& Johnson, B. T. (2011). Intergroup consensus/disagreement in support of group-based hierarchy: An examination of socio-structural and psycho-cultural factors. Psychological Bulletin, 137, 1029.

Leiserowitz, A., Maibach, E., Roser-Renouf, C., \& Hmielowski, J. D. (2012). Extreme weather, climate \& preparedness in the american mind. Yale University and George Mason University. New Haven.

Leiserowitz, A., Maibach, E., Roser-Renouf, C., Feinberg, G., \& Rosenthal, S. (2015). Climate Change in the American Mind: March, 2015. Yale Project on Climate Change Communication. Yale University and George Mason University, New Haven.

Leiserowitz, A., Maibach, E., Roser-Renouf, C., Rosenthal, S., Cutler, M., \& Kotcher, J. (2017). Climate change in the American mind: October 2017. Yale University and George Mason University. New Haven, CT: Yale Program on Climate Change Communication.

Leone, L., \& Chirumbolo, A. (2008). Conservatism as motivated avoidance of affect: Need for affect scales predict conservatism measures. Journal of Research in Personality, 42, 755-762.

Levine, A. S., \& Kline, R. (2017). A new approach for evaluating climate change communication. Climatic change, 142, 301-309.

Little, T. D., Cunningham, W. A., Shahar, G., \& Widaman, K. F. (2002). To parcel or not to parcel: Exploring the question, weighing the merits. Structural equation modeling, 9, 151-173. 
Liu, J. H., Huang, L. L., \& McFedries, C. (2008). Cross-sectional and longitudinal differences in social dominance orientation and right wing authoritarianism as a function of political power and societal change. Asian Journal of Social Psychology, 11, 116-126.

Liu, J. H., \& Sibley, C. G. (2012). Hope for the future? Understanding self-sacrifice in the face of global warming among young citizens of the world. Analyses of Social Issues and Public Policy, 12, 190-203.

Lorenzoni, I., Nicholson-Cole, S., \& Whitmarsh, L. (2007). Barriers perceived to engaging with climate change among the UK public and their policy implications. Global environmental change, $17,445-459$.

MacCallum, R. C., Browne, M. W., \& Sugawara, H. M. (1996). Power analysis and determination of sample size for covariance structure modeling. Psychological methods, 1, 130.

Macdiarmid, J., Douglas, F., \& Campbell, J. (2016). Eating like there's no tomorrow: Public awareness of the environmental impact of food and reluctance to eat less meat as part of a sustainable diet. Appetite, 96, 487.

Maibach, E., Leiserowitz, A., Roser-Renouf, C., Myers, T., Rosenthal, S. \& Feinberg, G. (2015). The Francis effect: How Pope Francis changed the conversation about global warming. George Mason University and Yale University. Fairfax, VA: George Mason University Center for Climate Change Communication

Malka, A., Krosnick, J. A. \& Langer, G. (2009). The association of knowledge with concern about global warming: trusted information sources shape public thinking. Risk Analysis, 29, 33647.

Marteau, T. M., Sowden, A. J., \& Armstrong, D. (1998). Implementing research findings into practice: beyond the information deficit model. In A. Haines \& A. Donald (Eds.), Getting research findings into practice (pp. 36-42). London: BMJ Publishing Group.

Martin, J. G., \& Westie, F. R (1959). The tolerant personality. American Sociological Review, 24, $521,528$.

McCright, A. M., \& Dunlap, R. E. (2000). Challenging global warming as a social problem: An analysis of the conservative movement's counter-claims. Social problems, 47, 499-522.

McCright, A. M., \& Dunlap, R. E. (2003). Defeating Kyoto: The Conservative Movement's Impact on U.S. Climate Change Policy. Social Problems, 50, 348-73. 
McCright, A. M., \& Dunlap, R. E. (2011). The politicization of climate change and polarization in the American public's views of global warming, 2001-2010. The Sociological Quarterly, 52, 155-194.

Milfont, T. L. (2009). The effects of social desirability on self-reported environmental attitudes and ecological behaviour. The Environmentalist, 29, 263-269.

Milfont, T. L., \& Duckitt, J. (2010). The environmental attitudes inventory: A valid and reliable measure to assess the structure of environmental attitudes. Journal of Environmental Psychology, 30, 80-94.

Milfont, T. L., Richter, I., Sibley, C. G., Wilson, M. S., \& Fischer, R. (2013). Environmental consequences of the desire to dominate and be superior. Personality and Social Psychology Bulletin, 39, 1127-1138.

Milfont, T. L., Sibley, C. G. (2014). The hierarchy enforcement hypothesis of environmental exploitation: A social dominance perspective. Journal of Experimental Social Psychology, 55, 188-193.

Milfont, T. L., \& Sibley, C. G. (2016). Empathic and social dominance orientations help explain gender differences in environmentalism: A one-year Bayesian mediation analysis. Personality and Individual Differences, 90, 85-88.

Milfont, T. L., Milojev, P., Greaves, L. M., \& Sibley, C. G. (2015). Socio-structural and psychological foundations of climate change beliefs. New Zealand Journal of Psychology, 44, $17-30$.

Milfont, T. L., Bain, P. G., Kashima, Y., Corral-Verdugo, V., Pasquali, C., Johansson, L. O., ... \& Bilewicz, M. (2018). On the relation between social dominance orientation and environmentalism: A 25-nation study. Social Psychological and Personality Science, 9, 802814.

Milfont, T. L., Wilson, M. S., \& Sibley, C. G. (2017). The public's belief in climate change and its human cause are increasing over time. PLOS ONE, 12.

Milojev, P., Osborne, D., \& Sibley, C. G. (2014). Default statistical standards and modelling decisions for NZAVS data analysis. NZAVS Technical Documents.

Minson, J. A., \& Monin, B. (2012). Do-gooder derogation: Disparaging morally motivated minorities to defuse anticipated response. Social Psychological and Personality Science, 3, 200-207. 
Muthén, L. K. \& Muthén, B. O. (1998-2010). Mplus user's guide. Sixth edition. Los Angeles, CA: Muthén \& Muthén.

Nederhof, A. J. (1985). Methods of coping with social desirability bias: A review. European Journal of Social Psychology, 15, 263-280.

New Zealand Gazette. (2011). The climate change response (2050 emissions target) notice 2011. The Gazette, 41, 987. https://gazette.govt.nz/notice/id/2011-go2067

Noe, F. P., \& Snow, R. (1990). The new environmental paradigm and further scale analysis. The Journal of Environmental Education, 21, 20-26.

Nunnally, J. C. (1978). Psychometric Theory. New York: McGraw-Hill

O’Rourke, D., \& Connolly, S. (2003). Just oil? The distribution of environmental and social impacts of oil production and consumption. Annual Review of Environmental Resources, 28, 587-617.

Oreskes, N., \& Conway, E. M. (2010). Merchants of doubt. Nature, 465, 686.

Osborne, D., Milojev, P., \& Sibley, C. G. (2017). Authoritarianism and national identity: examining the longitudinal effects of SDO and RWA on nationalism and patriotism. Personality and Social Psychology Bulletin, 43, 1086-1099.

Overbeck, J. R., Jost, J. T., Mosso, C. O., \& Flizik, A. (2004). Resistant versus acquiescent responses to ingroup inferiority as a function of social dominance orientation in the USA and Italy. Group Processes \& Intergroup Relations, 7, 35-54.

Owens, S., \& Driffill, L. (2008). How to change attitudes and behaviours in the context of energy. Energy Policy, 36, 4412-4418.

Panno, A., Giacomantonio, M., Carrus, G., Maricchiolo, F., Pirchio, S., \& Mannetti, L. (2018). Mindfulness, Pro-environmental Behavior, and Belief in Climate Change: The Mediating Role of Social Dominance. Environment and Behaviour, 50, 864-888.

Parliamentry Comissioner for the Environment. (2016). Climate change and agriculture: Understanding the biological greenhouse gases. New Zealand.

Pellow, D. N. (2000). Environmental inequality formation: Toward a theory of environmental justice. American Behavioural Scientist, 43, 581-601

Peterson, B. E., Doty, R. M., \& Winter, D. G. (1993). Authoritarianism and attitudes toward contemporary social issues. Personality and Social Psychology Bulletin, 19, 174-184. 
Pirages, D. C., \& Ehrlich, P. R. (1974). Ark II; social response to environmental imperatives [by] Dennis C. Pirages [and] Paul R. Ehrlich.

Polanin, J. R. (2013). Addressing the issue of meta-analysis multiplicity in education and psychology. [Doctoral dissertation]. Loyola University, Chicago.

Poore, J., \& Nemecek, T. (2018). Reducing food's environmental impacts through producers and consumers. Science, 360, 987-992.

Posthuma, L., Bjørn, A., Zijp, M. C., Birkved, M., Diamond, M. L., Hauschild, M. Z., ... \& Van de Meent, D. (2014). Beyond safe operating space: finding chemical footprinting feasible. Environmental Science \& Technology, 48, 6057-6059.

Potthast, M. (1993). Confirmatory factor analysis of ordered categorical variables with large models. British Journal of Mathematical \& Statistical Psychology, 46, 273-286.

Pratto, F., Cidam, A., Stewart, A. L., Zeineddine, F. B., Aranda, M., Aiello, A., ... \& Eicher, V. (2013). Social dominance in context and in individuals: Contextual moderation of robust effects of social dominance orientation in 15 languages and 20 countries. Social Psychological and Personality Science, 4, 587-599.

Pratto, F., Sidanius, J., Stallworth, L. M., Malle, B. F. (1994). Social dominance orientation: A personality variable predicting social and political attitudes. Journal of Personality and Social Psychology, 67, 741-763.

Pratto, F., Stallworth, L. M., \& Sidanius, J. (1997). The gender gap: Differences in political attitudes and social dominance orientation. British Journal of Social Psychology, 36, 49-68.

Queirós, A., Faria, D., \& Almeida, F. (2017). Strengths and limitations of qualitative and quantitative research methods. European Journal of Education Studies, 3, 369-387.

QSR International Pty Ltd. (2017). NVivo qualitative data analysis Software; Version 11.4.2.

Rahman, M. S. (2017). The Advantages and Disadvantages of Using Qualitative and Quantitative Approaches and Methods in Language "Testing and Assessment" Research: A Literature Review. Journal of Education and Learning, 6, 102-112.

Ray, J. J., \& Wilson, R. S. (1976). Social conservatism in Australia. Australian and New Zealand Journal of Statistics, 12, 254-257.

Rea, L. M., \& Parker, R. A. (1992). Designing and conducting survey research. San Francisco: Jossey-Boss. 
Reese, G. (2012). When authoritarians protect the earth - Authoritarian submission and proenvironmental beliefs: A pilot study in Germany. Ecopsychology, 4, 232-236.

Reese, G., \& Junge, E. A. (2017). Keep on rockin'in a (plastic-) free world: Collective efficacy and pro-environmental intentions as a function of task difficulty. Sustainability, 9, 1-13.

Reynolds, W. M. (1982). Development of reliable and valid short forms of the Marlowe-Crowne Social Desirability Scale. Journal of Clinical Psychology, 38, 119-125.

Rhodes, R. E., \& de Bruijn, G. (2013). How big is the physical activity intention-behaviour gap? A meta-analysis using the action control framework. British Journal of Health Psychology, 18, 296-309.

Ripple, W. J., Wolf, C., Newsome, T. M., Galetti, M., Alamgir, M., Crist, E., ... \& 15,364 scientist signatories from 184 countries. (2017). World scientists' warning to humanity: a second notice. BioScience, 67, 1026-1028.

Rissel, C., Campbell, F., Ashley, B., \& Jackson, L. (2002). Driver road rule knowledge and attitudes towards cyclists. Australian Journal of Primary Health, 8, 66-69.

Ritchie, H., \& Roser, M. (2018). Fossil Fuels. OurWorldInData.org. Accessed via https://ourworldindata. org/fossil-fuels

Roccato, M., \& Ricolfi, L. (2005). On the correlation between right-wing authoritarianism and social dominance orientation. Basic and Applied Social Psychology, 27, 187-200.

Rosendaal, F. R., \& Reitsma, P. H. (2014) Meta-analysis. Journal of Thrombosis and Haemostasis, 12, 1009 .

Rosenthal, R. (1979). The file drawer problem and tolerance for null results. Psychological Bulletin, $86,638$.

Ruddiman, W. F., Ellis, E. C., Kaplan, J. O., \& Fuller, D. Q. (2015). Defining the epoch we live in. Science, 348, 38-39.

Saad, A. (2018). Pathways of Harm: The Consequences of Trump's Withdrawal from the Paris Climate Agreement. Environmental Justice, 11, 47-51.

Sandelowski, M. (1995). Sample size in qualitative research. Research in Nursing \& Health, 18, 179183.

Satorra, A. \& Bentler, P.M. (2010). Ensuring positiveness of the scaled difference chi-square test statistic. Psychometrika, 75, 243-248. 
Scammacca, N., Roberts, G., \& Stuebing, K. K. (2014). Meta-analysis with complex research designs: Dealing with dependence from multiple measures and multiple group comparisons. Review of Educational Research, 84, 328-364.

Scarborough, P., Appleby, P. N., Mizdrak, A., Briggs, A. D., Travis, R. C., Bradbury, K. E., \& Key, T. J. (2014). Dietary greenhouse gas emissions of meat-eaters, fish-eaters, vegetarians and vegans in the UK. Climate Change, 125, 179-192.

Schlosberg, D., \& Collins, L. B. (2014). From environmental to climate justice: Climate change and the discourse of environmental justice. WIREs Climate Change, 5, 359-374.

Schuldt, J. P., Konrath, S. H. \& Schwarz, N. (2011). “Global warming” or "climate change”? Whether the planet is warming depends on question wording. Public Opinion Quarterly, 75, 115-124.

Schuldt, J. P., Pearson, A. R., Romero-Canyas, R., \& Larson-Konar, D. (2017). Brief exposure to Pope Francis heightens moral beliefs about climate change. Climatic Change, 141, 167-177.

Schultz, P. W., \& Stone, W. F. (1994). Authoritarianism and attitudes toward the environment. Environment and Behavior, 26, 25-37.

Schwartz, S. H. (1992). Universals in the content and structures of values: theoretical advances and empirical tests in 20 countries. In Advances in Experimental Psychology, Vol. 25, ed. M Zanna, pp. 1-65. Orlando, FL: Academic

Scovronick, N., Budolfson, M. B., Dennig, F., Fleurbaey, M., Siebert, A., Socolow, R. H., ... \& Wagner, F. (2017). Impact of population growth and population ethics on climate change mitigation policy. Proceedings of the National Academy of Sciences, 114, 12338-12343.

Seacat, J. D., \& Boileau, N. (2018). Demographic and community-level predictors of recycling behaviour: A statewide, assessment. Journal of Environmental Psychology, 56, 12-19.

Sears, D. O., Haley, H., \& Henry, P. J. (2008). Cultural diversity and sociopolitical attitudes at college entry. In J. Sidanius, S. Levin, C. van Laar, \& D. O. Sears (Eds.), The diversity challenge (pp. 65-99). New York, NY: Russell Sage.

Shurpali, N., Agarwal, A. K., \& Srivastava, V. K. (2019). Introduction to Greenhouse Gas Emissions. In Greenhouse Gas Emissions (pp. 1-5). Singapore: Springer.

Sibley, C. G. (2009 - 2015). New Zealand Attitudes and Values Study. Unpublished raw data. 
Sibley, C. G. (2014) Sampling procedure and sample details for the New Zealand Attitudes and Values Study. NZAVS Technical Documents.

Sibley, C. G., \& Wilson, M. S. (2007). Political attitudes and the ideology of equality: Differentiating support for liberal and conservative political parties in New Zealand. New Zealand Journal of Psychology, 36, 72.

Sidanius, J., Cotterill, S., Sheehy-Skeffington, J., Kteily, N., \& Carvacho, H. (2016). Social dominance theory: Explorations in the psychology of oppression. In Sibley, C. G. \& Barlow, F. K. (Eds.), The Cambridge Handbook of the Psychology of Prejudice.

Sidanius, J., Kteily, N., Sheehy-Skeffington, J., Ho, A. K., Sibley, C., \& Duriez, B. (2013). You're inferior and not worth our concern: The interface between empathy and social dominance orientation. Journal of Personality, 81, 313-323.

Sidanius, J., Levin, S., Van Laar, C., \& Sears, D. O. (2008). The diversity challenge: Social identity and intergroup relations on the college campus. Russell Sage Foundation.

Sidanius, J., \& Pratto, F. (1993). The inevitability of oppression and the dynamics of social dominance. Prejudice, politics, and the American dilemma, 173-211.

Sidanius, J., \& Pratto, F. (1999). Social Dominance: An Intergroup Theory of Social Hierarchy and Oppression. Cambridge: Cambridge University Press.

Sidanius, J., Pratto, F., \& Mitchell, M. (1994). In-group identification, social dominance orientation, and differential intergroup social allocation. The Journal of Social Psychology, 134, $151-167$

Simmonds, M. (2015). Quantifying the risk of error when interpreting funnel plots. Systematic Reviews, 4, 24.

Singer, M. (2018). Climate Change and Social Inequality: The Health and Social Costs of Global Warming. Routledge.

Smil, V. (2017). Energy Transitions: Global and National Perspectives. \& BP Statistical Review of World Energy. http://vaclavsmil.com/2016/12/14/energy-transitions-global-and-nationalperspectives-second-expanded-and-updated-edition/ ; http://www.bp.com/statisticalreview

Smith, N., \& Leiserowitz, A. (2014). The role of emotion in global warming policy support and opposition. Risk Analysis, 34, 937-948. 
Spence, A., Poortinga, W., \& Pidgeon, N. (2012). The psychological distance of climate change. Risk analysis, 32, 957-972.

Stanley, S., Millin, P., Mickleson, K., \& Milfont, T. L. (2018, August 29). Not here, not to us, maybe not at all: The psychological distance of climate change in relation to environmental engagement. https://doi.org/10.31234/osf.io/qt5nj

Stanley, S. K., Milfont, T. L., Wilson, M. S., \& Sibley, C. G. (2019). The influence of social dominance orientation and right-wing authoritarianism on environmentalism: A five-year cross-lagged analysis. PLoS ONE, 14, e0219067. https://doi.org/10.1371/journal.pone.0219067.

Stanley, S. K., \& Wilson, M. S. (2019). Meta-analysing the association between social dominance orientation, authoritarianism, and attitudes on the environment and climate change. Journal of Environmental Psychology, 61, 46-56.

Stanley, S. K., Wilson, M. S., \& Milfont, T. L. (2017a). Exploring short-term longitudinal effects of right-wing authoritarianism and social dominance orientation on environmentalism. Personality and Individual Differences, 108, 174-177.

Stanley, S. K., Wilson, M. S., Sibley, C. G., \& Milfont, T. L. (2017b). Dimensions of social dominance and their associations with environmentalism. Personality and Individual Differences, 107, 228-236.

Steg, L. (2003). Can public transport compete with the private car? Itass Research, 27, 27-35.

Steg, L., \& Vlek, C. (2009). Encouraging pro-environmental behaviour: An integrative review and research agenda. Journal of Environmental Psychology, 29, 309-317.

Stern, P. C. (1992). Psychological dimensions of global environmental change. Annual Review of Psychology, 43, 269-301.

Stern, P. C. (2002). New Environmental Theories: Toward a Coherent Theory of Environmentally Significant Behavior. Journal of Social Issues, 56, 407-424.

Stevenson, K. T., Peterson, M. N., Bondell, H. D., Moore, S. E., \& Carrier, S. J. (2014). Overcoming scepticism with education: Interacting influences of worldview and climate change knowledge on perceived climate change risk among adolescents. Climatic Change, 126, 293304.

Stokes, B., Wike, R., \& Carle, J. (2015). Global concern about cliamte change, broad support for limiting emissions. Pew Research Center. 
Stoll-Kleemann, S., O’Riordan, T., \& Jaeger, C. C. (2001). The psychology of denial concerning climate mitigation measures: Evidence from Swiss focus groups. Global Environmental Change, 11, 107-117.

Stuff.co.nz (2016). Terms and Conditions: User submitted content and comments.

SurveyMonkey Inc. www.surveymonkey.com Location: San Mateo, California, USA https://www.stuff.co.nz/about-stuff/87006221/terms-and-conditions-user-submitted-contentand-comments.

Sutton, A. J., \& Higgins, J. P. (2008). Recent developments in meta-analysis. Statistics in medicine, $27,625-650$.

Taber, C. S., \& Lodge, M. (2006). Motivated skepticism in the evaluation of political beliefs. American Journal of Political Science, 50, 755-769.

Tetlock, P. E., Lupia, A., McCubbins, M. D., \& Popkin, S. L. (2000). Coping with trade-offs: Psychological constraints and political implications. Elements of reason: Cognition, choice, and the bounds of rationality, 239-263.

Thibodeau, R. (1998). Integrative complexity in reasoning about the natural environment: An investigation of Authoritarianism and Machiavellianism. [Master's thesis]. Carleton University, Canada.

Thomas, G. O., Fisher, R., Whitmarsh, L., Milfont, T. L., \& Poortinga, W. (2018). The impact of parenthood on environmental attitudes and behaviour: a longitudinal investigation of the legacy hypothesis. Population and Environment, 39, 261-276.

Trumbo, C. (1996). Constructing climate change: Claims and frames in US news coverage of an environmental issue. Public Understanding of Science, 5, 269-83.

Trump, D. J. (28 January, 2014).

https://twitter.com/realdonaldtrump/status/428414113463955457?lang=en

Tukey, J. W. (1949). Comparing individual means in the analysis of variance. Biometrics, 5, 99-114.

Turner, J. C., \& Reynolds, K. J. (2003). Why social dominance theory has been falsified. British Journal of Social Psychology, 42, 199-206.

United Nations. (2015). Report of the Conference of the Parties on its twenty-first session, held in Paris from 30 November to 13 December 2015. Addendum. Part two: Action taken by the 
Conference of the Parties at its twenty-first session. Bonn: United Nations Framework Convention on Climate Change.

Van Hiel, A., Cornelis, I., Roets, A., \& De Clercq, B. (2007). A comparison of various authoritarianism scales in Belgian Flanders. European Journal of Personality, 21, 149-168.

Van Hiel, A. V., \& Kossowska, M. (2007). Contemporary attitudes and their ideological representation in Flanders (Belgium), Poland, and the Ukraine. International Journal of Psychology, 42, 16-26.

Vail, K. E., \& Motyl, M. (2010). Support for diplomacy: Peacemaking and militarism as a unidimensional correlate of social, environmental, and political attitudes. Peace and Conflict, $16,29-57$.

van Valkengoed, A. M., \& Steg, L. (2019). Meta-analyses of factors motivating climate change adaptation behaviour. Nature Climate Change.

Velicer, W. F. (1978). Suppressor variables and the semipartial correlation coefficient. Educational and Psychological Measurement, 38, 953-958.

Verheggen, B., Strengers, B., Cook, J., van Dorland, R., Vringer, K., Peters, J., Visser, H. \& Meyer, L. (2014). Scientists' views about attribution of global warming. Environmental Science \& Technology, 48, 8963-8971.

Vittinghoff, E., Glidden, D. V., Shiboski, S. C., \& McCulloch, C. E. (2005). Regression methods in biostatistics: linear, logistic, survival and repeated measures models. Springer.

Wang, A. Y. (1999). Gender and nature: A psychological analysis of ecofeminism theory. Journal of Applied Social Psychology, 29, 2410-2424.

Warren, K. J. (1990). The power and promise of ecological feminism. Environmental Ethics, 12, $125-146$.

Wheaton, B., Muthén, B., Alwin, D., \& Summers, G. (1977). Assessing reliability and stability in panel models. Sociological Methodology, 8, 84-136.

Whitley, B. E. (1999). Right-wing authoritarianism, social dominance orientation, and prejudice. Journal of Personality and Social Psychology, 77, 126.

Wilson, M. S. (2003) Social dominance and ethical ideology: The end justifies the means? The Journal of Social Psychology, 143, 549-558. 
Wilson, M. S., \& Sibley, C. G. (2013). Social dominance orientation and right-wing authoritarianism: Additive and interactive effects on political conservatism. Political Psychology, 34, 277-284.

Wolsko, C., Ariceaga, H., \& Seiden, J. (2016). Red, white, and blue enough to be green: effects of moral framing on climate change attitudes and conservation behaviors. Journal of Experimental Social Psychology, 65, 7-19

World Resources Institute. (2014). Climate Analysis Indicators Tool (CAIT) Version 2.0. Washington, DC: World Resources Institute. Retrieved 2017-06-12.

Wynes, S., \& Nicholas, K. A. (2017). The climate mitigation gap: education and government recommendations miss the most effective individual actions. Environmental Research Letters, 12, 074024.

Yung, Y., \& Bentler, P. (1994). Bootstrap-corrected ADF test statistics in covariance structure analysis. British Journal of Mathematical \& Statistical Psychology, 47, 63-84.

Zakrisson, I. (2005). Construction of a short version of the Right-Wing Authoritarianism (RWA) scale. Personality and Individual Differences, 39, 863-872.

Zhao, H., Zhang, H., Xu, Y., Lu, J., \& He, W. (2018). Relation between awe and environmentalism: The role of social dominance orientation. Frontiers in psychology, 9. 


\section{Appendix A}

Table A.

Summary of studies included in the meta-analyses of zero-order correlations (Study 1A).

\begin{tabular}{|c|c|c|c|c|c|c|c|c|}
\hline \multirow[b]{2}{*}{ Authors } & \multirow[b]{2}{*}{ Environment } & \multicolumn{2}{|l|}{ Measures } & \multicolumn{2}{|c|}{ Correlation } & \multicolumn{3}{|c|}{ Sample details } \\
\hline & & SDO & RWA & SDO & RWA & N / Type & $\begin{array}{l}\% \\
\text { fema } \\
\text { le }\end{array}$ & Country \\
\hline $\begin{array}{l}\text { Amérigo, Palavencinos, García, Román \& } \\
\text { Trizano-Hermosilla (2016) }\end{array}$ & 5 & Full scale & - & -.23 & - & $262 \mathrm{~S}$ & 60 & Chile \\
\hline Caddick (2016) & $\begin{array}{l}1 \\
3 \\
\text { Overall }\end{array}$ & - & $\mathrm{ACT}$ & - & $\begin{array}{l}-.32 \\
-.33 \\
-.32 \\
\end{array}$ & $445 \mathrm{G}$ & 64 & US \\
\hline $\begin{array}{l}\text { Choma, Hanoch, Gummerum \& Hodson } \\
\text { (2013) }\end{array}$ & 6 & Full scale & Shortened & -.29 & -.26 & $387 \mathrm{G}$ & 59 & US \\
\hline Currie \& Choma (unpublished)* & $\begin{array}{l}\text { S1: } 5 \\
\text { S2: } 5\end{array}$ & $\begin{array}{l}\text { Full scale } \\
\text { Full scale }\end{array}$ & $\begin{array}{l}\text { Shortened } \\
\text { Shortened }\end{array}$ & $\begin{array}{l}-.20 \\
-.24\end{array}$ & $\begin{array}{l}-.23 \\
.01\end{array}$ & $\begin{array}{l}200 \mathrm{~S} \\
150 \mathrm{G}\end{array}$ & $\begin{array}{l}83 \\
50\end{array}$ & $\begin{array}{l}\text { Canada } \\
\text { US }\end{array}$ \\
\hline Devine-Wright, Price \& Leviston (2015) & 1 & Shortened & ACT & -.22 & -.20 & $576 \mathrm{G}$ & 57 & Australia \\
\hline Dimdins, Sandgren \& Montgomery (2016)* & $\begin{array}{l}\text { S1: } 5 \\
\text { S2: } 5\end{array}$ & $\begin{array}{l}\text { Full scale } \\
\text { Full scale }\end{array}$ & $\begin{array}{l}\text { Zakrisson } \\
\text { Zakrisson }\end{array}$ & $\begin{array}{r}-.36 \\
-.29\end{array}$ & $\begin{array}{l}-.21 \\
-.01 \\
\end{array}$ & $\begin{array}{l}320 \mathrm{~S} \\
264 \mathrm{~S}\end{array}$ & $\begin{array}{l}67 \\
76 \\
\end{array}$ & $\begin{array}{l}\text { Sweden } \\
\text { Latvia }\end{array}$ \\
\hline Duckitt, Bizumic, Krauss \& Heled (2010) & 4 & - & $\mathrm{ACT}$ & - & -.34 & $209 \mathrm{~S}$ & 76 & $\mathrm{NZ}$ \\
\hline Häkkinen \& Akrami (2014) & S1: 1 & Full scale & Zakrisson & -.53 & -.33 & $135 \mathrm{G}$ & 68 & Sweden \\
\hline & $\begin{array}{l}\text { S2a: } 1 \\
\text { S2b: } 1\end{array}$ & $\begin{array}{l}\text { Shortened } \\
\text { Shortened }\end{array}$ & $\begin{array}{l}\text { Zakrisson } \\
\text { Zakrisson }\end{array}$ & $\begin{array}{l}-.35 \\
-.47\end{array}$ & $\begin{array}{l}-.20 \\
-.40\end{array}$ & $\begin{array}{l}53 \mathrm{G} \\
48 \mathrm{G}\end{array}$ & $\begin{array}{l}60 \\
60\end{array}$ & $\begin{array}{l}\text { Sweden } \\
\text { Sweden }\end{array}$ \\
\hline Hoffarth \& Hodson (2016) & $\begin{array}{l}1 \\
2 \\
3 \\
5 \\
\text { Overall } \\
\end{array}$ & Full scle & Zakrisson & $\begin{array}{l}-.35 \\
-.50 \\
-.57 \\
-.40 \\
-.42 \\
\end{array}$ & $\begin{array}{l}-.40 \\
-.43 \\
-.59 \\
-.33 \\
-.40 \\
\end{array}$ & $384 \mathrm{G}$ & 46 & US \\
\hline Hoffarth \& Hodson unpublished* & S1: 1 & SDO7 & Shortened & -.37 & -.44 & $200 \mathrm{G}$ & 51 & US \\
\hline
\end{tabular}




\begin{tabular}{|c|c|c|c|c|c|c|c|c|}
\hline & $\begin{array}{l}\text { S2: } 1 \\
3 \\
6 \\
\text { Overall }\end{array}$ & SDO7 & Shortened & $\begin{array}{l}-.42 \\
-.62 \\
-.48 \\
-.49 \\
\end{array}$ & $\begin{array}{l}-.45 \\
-.70 \\
-.49 \\
-.52 \\
\end{array}$ & $304 \mathrm{G}$ & 48 & US \\
\hline Hoot (thesis)* & 5 & Full scale & - & -.12 & - & $103 \mathrm{G}$ & 34 & US \\
\hline 'Howison (2005) & 4 & Full scale & Full scale & -.24 & -.22 & $116 / 128 \mathrm{G}$ & 59 & $\mathrm{NZ}$ \\
\hline Jackson, Bitacola, Janes \& Esses (2013) & 5 & Full scale & - & -.59 & - & $72 \mathrm{~S}$ & 68 & Canada \\
\hline Jylha \& Akrami (2013) unpublished* & $\begin{array}{l}1 \\
5 \\
6 \\
\text { Overall } \\
\end{array}$ & Full scale & Zakrisson & $\begin{array}{l}-.37 \\
-.31 \\
-.21 \\
-.28 \\
\end{array}$ & $\begin{array}{l}-.23 \\
-.16 \\
-.11 \\
-.15 \\
\end{array}$ & $102 \mathrm{G}$ & 71 & Sweden \\
\hline Jylhä \& Akrami (2015) & $\begin{array}{l}1 \\
5 \\
\text { Overall } \\
\end{array}$ & SOD7 & - & $\begin{array}{l}-.37 \\
-.30 \\
-.34 \\
\end{array}$ & - & $221 \mathrm{G}$ & 66 & Sweden \\
\hline Jylha \& Akrami (2015) unpublished* & $\begin{array}{l}1 \\
5 \\
6 \\
\text { Overall }\end{array}$ & SDO7 & - & $\begin{array}{l}-.53 \\
-.44 \\
-.31 \\
-.43\end{array}$ & - & $222 \mathrm{G}$ & 68 & Sweden \\
\hline Jylhä, Cantal, Akrami \& Milfont (2016) & 1 & SDO7 & - & -.25 & - & $367 \mathrm{G}$ & 59 & Brazil \\
\hline \multirow[t]{3}{*}{ Kemmelmeier (2016)* } & S1: 4 & - & Shortened & - & .11 & $126 \mathrm{G}$ & 44 & US \\
\hline & S2: 4 & - & Shortened & - & .25 & $90 \mathrm{G}$ & 51 & US \\
\hline & S3: 4 & - & Shortened & - & .08 & $79 \mathrm{G}$ & 49 & US \\
\hline Leftcourt (1996) & 5 & - & Full scale & - & -.14 & $304 \mathrm{~S}$ & 57 & Canada \\
\hline \multirow[t]{3}{*}{ Leung, Koh \& Tam (2015) } & $\begin{array}{l}\text { S1:2 } \\
5 \\
\text { Overall }\end{array}$ & Shortened & - & $\begin{array}{l}-.18 \\
-.23 \\
-.20\end{array}$ & - & $309 \mathrm{~S}$ & 69 & Singapore \\
\hline & $\begin{array}{l}\text { S2: } 2 \\
5 \\
\text { Overall }\end{array}$ & Full scale & - & $\begin{array}{l}-.29 \\
-.26 \\
-.28\end{array}$ & - & $99 \mathrm{~S}$ & 55 & Australia \\
\hline & S3: 2 & Full scale & - & -.41 & & $251 \mathrm{G}$ & & US \\
\hline
\end{tabular}




\begin{tabular}{|c|c|c|c|c|c|c|c|c|}
\hline & $\begin{array}{l}5 \\
\text { Overall }\end{array}$ & & & $\begin{array}{l}-.46 \\
-.42\end{array}$ & & & & \\
\hline \multirow[t]{2}{*}{ McDermott (2007) } & $\begin{array}{l}\text { S1:2 } \\
5 \\
\text { Overall }\end{array}$ & - & Shortened & - & $\begin{array}{l}.03 \\
-.35 \\
-.16\end{array}$ & $240 \mathrm{G}$ & 59 & Argentina \\
\hline & $\begin{array}{l}\mathrm{S} 2: 2 \\
5 \\
\text { Overall }\end{array}$ & - & Shortened & - & $\begin{array}{l}-.20 \\
-.39 \\
-.30\end{array}$ & $105 \mathrm{G}$ & 100 & UK \\
\hline \multirow[t]{2}{*}{ McFarland (unpublished)* } & $\begin{array}{l}\text { S1: } 1 \\
3 \\
5 \\
\text { Overall }\end{array}$ & Full scale & Zakrisson & $\begin{array}{l}-.49 \\
-.56 \\
-.28 \\
-.46\end{array}$ & $\begin{array}{l}-.52 \\
-.54 \\
-.19 \\
-.44\end{array}$ & $\begin{array}{l}1767 \mathrm{G} \\
1852 \mathrm{G} \\
1276 \mathrm{G} \\
1632 \mathrm{G}\end{array}$ & 41 & Global \\
\hline & S2: 1 & Shortened & Zakrisson & -.50 & -.62 & $385 \mathrm{G}$ & 61 & US \\
\hline \multirow{2}{*}{$\begin{array}{l}{ }^{+} \text {Milfont, Richter, Sibley, Wilson \& Fischer } \\
\text { (2013) }\end{array}$} & S3: 5 & Shortened & Shortened & -.24 & -.21 & $150 \mathrm{~S}$ & 69 & NZ \\
\hline & $\begin{array}{l}\text { S4: } 1 \\
4 \\
5 \\
\text { Overall }\end{array}$ & Shortened & Shortened & $\begin{array}{l}-.36 \\
-.36 \\
-.42 \\
-.37 \\
\end{array}$ & $\begin{array}{l}-.38 \\
-.36 \\
-.36 \\
-.36 \\
\end{array}$ & $3849 \mathrm{G}$ & 61 & NZ \\
\hline \multirow[t]{2}{*}{ Peterson, Doty \& Winter (1993) } & $\begin{array}{l}\text { S2: } 2 \\
3 \\
5 \\
\text { Overall }\end{array}$ & - & Full scale & - & $\begin{array}{l}-.06 \\
-.32 \\
-.11 \\
-.15\end{array}$ & $278 \mathrm{~S}$ & 67 & US \\
\hline & S3: 3 & - & Full scale & - & -.49 & $170 \mathrm{~S}$ & 56 & US \\
\hline Peterson, Smirles \& Wentworth (1997)* & 2 & - & Full scale & - & -.15 & $193 \mathrm{~S}$ & 50 & US \\
\hline $\begin{array}{l}\text { Richert, Boschetti, Walker, Price \& Grigg } \\
(2017)^{*}\end{array}$ & $\begin{array}{l}1 \\
5 \\
\text { Overall }\end{array}$ & Shortened & Shortened & $\begin{array}{l}-.41 \\
-.43 \\
-.42\end{array}$ & $\begin{array}{l}-.31 \\
-.15 \\
-.23\end{array}$ & $130 \mathrm{G}$ & 45 & Australia \\
\hline Sabbagh (2005) & 2 & Other & - & -.31 & - & $3331 \mathrm{~S}$ & 44 & Germany \\
\hline Schultz \& Stone (1994) & $\begin{array}{l}\text { S1: } 2 \\
\text { S2: } 5\end{array}$ & - & $\begin{array}{l}\text { Shortened } \\
\text { Full scale }\end{array}$ & $\begin{array}{l}- \\
-\end{array}$ & $\begin{array}{l}-.51 \\
-.54\end{array}$ & $\begin{array}{l}80 \mathrm{G} \\
87 \mathrm{~S}\end{array}$ & $\begin{array}{l}40 \\
46\end{array}$ & $\begin{array}{l}\text { US } \\
\text { US }\end{array}$ \\
\hline
\end{tabular}




\begin{tabular}{|c|c|c|c|c|c|c|c|c|}
\hline Sibley \& Wilson (2007) & $\begin{array}{l}\mathrm{S} 1: 4 \\
\mathrm{~S} 2: 4\end{array}$ & $\begin{array}{l}\text { Shortened } \\
\text { Full scale }\end{array}$ & $\begin{array}{l}\text { Shortened } \\
\text { Shortened }\end{array}$ & $\begin{array}{l}-.25 \\
-.29\end{array}$ & $\begin{array}{l}-.32 \\
-.36\end{array}$ & $\begin{array}{l}259 \mathrm{~S} \\
146 \mathrm{~S}\end{array}$ & $\begin{array}{l}76 \\
64\end{array}$ & $\begin{array}{l}\text { NZ } \\
\text { NZ }\end{array}$ \\
\hline \multirow[t]{2}{*}{${ }^{+}$Sibley $(2009-2015)$} & $\begin{array}{l}\text { S1: } 1 \\
2 \\
5 \\
\text { Overall }\end{array}$ & Shortened & Shortened & $\begin{array}{l}-.20 \\
-.22 \\
-.19 \\
-.21\end{array}$ & $\begin{array}{l}-.07 \\
-.07 \\
.01 \\
-.06\end{array}$ & $\begin{array}{l}6301 / 6302 \mathrm{G} \\
6320 \\
6376 \\
6371 \mathrm{G}\end{array}$ & 60 & NZ \\
\hline & $\begin{array}{l}\text { S2: } 1 \\
5 \\
6 \\
\text { Overall } \\
\end{array}$ & Shortened & Shortened & $\begin{array}{l}-.33 \\
-.24 \\
-.32 \\
-.28 \\
\end{array}$ & $\begin{array}{l}-.27 \\
-.10 \\
-.23 \\
-.17 \\
\end{array}$ & $\begin{array}{l}10263 / 10260 \\
10343 / 10341 \\
10226 / 10223 \\
10277 / 10274 \\
\end{array}$ & 63 & NZ \\
\hline $\begin{array}{l}\text { Stevenson, Peterson, Bondell, Moore \& } \\
\text { Carrier (2014) }\end{array}$ & $\begin{array}{l}1 \\
6 \\
\text { Overall }\end{array}$ & Other & - & $\begin{array}{l}-.23 \\
-.18 \\
-.21\end{array}$ & - & $378 \mathrm{~S}$ & 55 & US \\
\hline Thibodeau (1998) & 5 & - & Full scale & - & .05 & $110 \mathrm{~S}$ & 50 & US \\
\hline Vail \& Motyl (2010) & $\begin{array}{l}2 \\
5 \\
\text { Overall }\end{array}$ & Full scale & Full scale & $\begin{array}{l}-.37 \\
-.35 \\
-.36\end{array}$ & $\begin{array}{l}-.29 \\
-.30 \\
-.29\end{array}$ & $231 \mathrm{~S}$ & 57 & Australia \\
\hline \multirow[t]{3}{*}{ Van Heil \& Kossowska (2007) } & $\begin{array}{l}\text { S1: } 2 \\
5 \\
\text { Overall }\end{array}$ & Other & - & $\begin{array}{l}-.47 \\
-.32 \\
-.37\end{array}$ & - & $176 \mathrm{G}$ & 39 & Belgium \\
\hline & $\begin{array}{l}\text { S2: } 2 \\
5 \\
\text { Overall }\end{array}$ & Other & - & $\begin{array}{l}0.00 \\
-.15 \\
-.10\end{array}$ & - & $93 \mathrm{G}$ & 38 & Poland \\
\hline & $\begin{array}{l}\text { S3: } 2 \\
5 \\
\text { Overall }\end{array}$ & Other & - & $\begin{array}{l}-.27 \\
-.26 \\
-.26 \\
\end{array}$ & & & & \\
\hline Van Hiel, Cornelis, Roets \& Clercq (2007) & 4 & - & Full scale & - & -.39 & $476 \mathrm{G}$ & 43 & Belgium \\
\hline Wang (1999) & $\begin{array}{l}\text { S1: } 5 \\
\text { S2: } 5\end{array}$ & Full scale & Full scale & $\begin{array}{l}-.38 \\
-.01\end{array}$ & $\begin{array}{l}-.36 \\
-.17\end{array}$ & $\begin{array}{l}97 \mathrm{~S} \\
60 \mathrm{~S}\end{array}$ & $\begin{array}{l}100 \\
0\end{array}$ & $\begin{array}{l}\text { US } \\
\text { US }\end{array}$ \\
\hline +Wilson (1998) & 5 & Full scale & - & -.21 & - & $245 \mathrm{~S}$ & 70 & NZ \\
\hline +Wilson \& Evers (2004) & 4 & Full scale & Full scale & -.31 & -.38 & $161 / 205 \mathrm{~S}$ & 71 & NZ \\
\hline
\end{tabular}




\begin{tabular}{|c|c|c|c|c|c|c|c|c|}
\hline${ }^{+}$Wilson (2005a) & 4 & Shortened & Shortened & -.22 & -.28 & $465 \mathrm{~S}$ & 70 & $\mathrm{NZ}$ \\
\hline \multirow{15}{*}{${ }^{+}$Wilson (2013) } & S1: 1 & Shortened & Full scale & -.10 & -.14 & $230 / 216 \mathrm{~S}$ & 71 & $\mathrm{NZ}$ \\
\hline & 2 & & & -.04 & -.03 & & & \\
\hline & 4 & & & -.12 & -.16 & & & \\
\hline & 5 & & & -.26 & -.23 & & & \\
\hline & Overall & & & -.12 & -.14 & & & \\
\hline & $\mathrm{S} 2: 1$ & Shortened & Full scale & -.17 & -.25 & $200 / 181 S$ & 75 & NZ \\
\hline & 2 & & & .04 & .02 & & & \\
\hline & 4 & & & -.09 & -.22 & & & \\
\hline & 5 & & & -.32 & -.39 & & & \\
\hline & Overall & & & -.15 & -.22 & & & \\
\hline & S3:1 & Shortened & Full scale & -.18 & -.27 & $226 \mathrm{~S}$ & 63 & $\mathrm{NZ}$ \\
\hline & 2 & & & .07 & -.01 & & & \\
\hline & 4 & & & -.01 & -.13 & & & \\
\hline & 5 & & & -.38 & -.33 & & & \\
\hline & Overall & & & -.14 & -.21 & & & \\
\hline \multirow[t]{10}{*}{${ }^{+}$Wilson (2014) } & S1: 1 & Full scale & Full scale & -.25 & -.23 & $356 \mathrm{~S}$ & 78 & $\mathrm{NZ}$ \\
\hline & 2 & & & -.02 & -.11 & & & \\
\hline & 4 & & & -.16 & -.17 & & & \\
\hline & 5 & & & -.30 & -.43 & & & \\
\hline & Overall & & & -.19 & -.23 & & & \\
\hline & S2: 1 & Full scale & Full scale & -.09 & -.13 & $285 \mathrm{~S}$ & 67 & NZ \\
\hline & 2 & & & -.04 & -.05 & & & \\
\hline & 4 & & & -.11 & -.24 & & & \\
\hline & 5 & & & -.28 & -.35 & & & \\
\hline & Overall & & & -.12 & -.18 & & & \\
\hline${ }^{+}$Wilson (2016) & 1 & Full scale & Full scale & -.17 & -.19 & $621 / 622 \mathrm{~S}$ & 67 & $\mathrm{NZ}$ \\
\hline${ }^{+}$Wilson (2017) & 5 & SDO7 & Full scale & -.36 & -.44 & $560 / 555 \mathrm{~S}$ & 73 & $\mathrm{NZ}$ \\
\hline \multirow[t]{2}{*}{${ }^{+}$Wilson $(2005 b)$} & 4 & Full scale & Full scale & -.31 & -.39 & $178 / 179 \mathrm{G}$ & 61 & $\mathrm{NZ}$ \\
\hline & 5 & & & -.11 & -.20 & & & \\
\hline
\end{tabular}




\begin{tabular}{|c|c|c|c|c|c|c|c|c|}
\hline & Overall & & & -.26 & -.34 & & & \\
\hline +Wilson (2005c) & 4 & Full scale & Full scale & -.23 & -.38 & $208 / 207 \mathrm{G}$ & 66 & $\mathrm{NZ}$ \\
\hline${ }^{+}$Wilson $(2005 d)$ & $\begin{array}{l}4 \\
5 \\
\text { Overall }\end{array}$ & Full scale & Full scale & $\begin{array}{l}.26 \\
-.31 \\
-.27\end{array}$ & $\begin{array}{l}-.20 \\
-.09 \\
-.17\end{array}$ & $204 \mathrm{G}$ & 60 & $\mathrm{NZ}$ \\
\hline
\end{tabular}

Note. Where studies are marked with $\mathrm{a}^{*}$, this indicates that we contacted the author(s) to obtain additional information to include findings from their study in our meta-analyses in Study 1A. A ${ }^{+}$indicates that the raw datasets were available for inclusion in our meta-analyses in Study 1B. The measures of environmentalism included are coded such that $1=$ climate change belief, $2=$ support for action, $3=$ environmentalist threat, $4=$ Green politics, 5 = pro-environmental attitudes, $6=$ climate change anxiety. The $\mathrm{G}$ after sample size indicates that the study used a general population sample, and the $\mathrm{S}$ a student sample. References for the studies included in the meta-analyses are in the Supplementary Material. US = United States, UK = United Kingdom, NZ = New Zealand. Where there are two sample sizes separated by a forward slash, this indicates that the sample size differed for each correlate, so the sample size is presented for SDO first then RWA $\left(\mathrm{N}_{\mathrm{SDO}} / \mathrm{N}_{\mathrm{RWA}}\right)$. 


\section{Appendix B}

Table B.

Summary of studies included in our meta-analyses of standardized regression weights.

\begin{tabular}{|c|c|c|c|c|c|c|}
\hline & \multicolumn{3}{|c|}{ Measures } & \multicolumn{2}{|c|}{ Beta weight } & \multirow[b]{2}{*}{ Adjusted $\mathrm{R}^{2}$} \\
\hline Authors & Environmentalism & SDO & RWA & SDO & RWA & \\
\hline Howison (2005) & 4 & Full scale & Full scale & -.16 & -.13 & .05 \\
\hline \multirow[t]{3}{*}{ Wilson \& Fischer (2013) } & 4 & & & -.25 & -.26 & .19 \\
\hline & 5 & & & -.33 & -.21 & .21 \\
\hline & Overall & & & -.27 & -.25 & \\
\hline \multirow{5}{*}{ Sibley $(2009-2015)$} & 2 & & & -.21 & -.03 & .04 \\
\hline & 5 & & & -.20 & .05 & .05 \\
\hline & Overall & & & -.21 & -.01 & \\
\hline & S2: 1 & Shortened & Shortened & -.28 & -.19 & .06 \\
\hline & 5 & & & -.23 & -.03 & .14 \\
\hline \multirow[t]{10}{*}{ Wilson (2013) } & S1: 1 & Shortened & Full scale & -.05 & -.11 & .02 \\
\hline & 2 & & & -.03 & -.01 & -.01 \\
\hline & 4 & & & -.04 & -.13 & .02 \\
\hline & 5 & & & -.20 & -.12 & .07 \\
\hline & Overall & & & -.07 & -.10 & \\
\hline & $\mathrm{S} 2: 1$ & Shortened & Full scale & -.10 & -.21 & .06 \\
\hline & 2 & & & .01 & .01 & -.01 \\
\hline & 4 & & & .004 & -.23 & .04 \\
\hline & 5 & & & -.12 & -.32 & .14 \\
\hline & Overall & & & -.07 & -.19 & \\
\hline
\end{tabular}




\begin{tabular}{|c|c|c|c|c|c|c|}
\hline & $\begin{array}{l}\text { S3:1 } \\
2 \\
4 \\
5 \\
\text { Overall }\end{array}$ & Shortened & Full scale & $\begin{array}{l}-.09 \\
.08 \\
.04 \\
-.30 \\
-.07 \\
\end{array}$ & $\begin{array}{l}-.23 \\
-.04 \\
-.15 \\
-.21 \\
-.18 \\
\end{array}$ & $\begin{array}{l}.09 \\
-.003 \\
.01 \\
.18\end{array}$ \\
\hline Wilson (2014) & $\begin{array}{l}\text { S1: } 1 \\
2 \\
4 \\
5 \\
\text { Overall }\end{array}$ & Full scale & Full scale & $\begin{array}{l}-.18 \\
.05 \\
-.09 \\
-.08 \\
-.09\end{array}$ & $\begin{array}{l}-.13 \\
-.14 \\
-.120 \\
-.38 \\
-.18\end{array}$ & $\begin{array}{l}.07 \\
.01 \\
.03 \\
.18\end{array}$ \\
\hline & $\begin{array}{l}\text { S2: } 1 \\
2 \\
4 \\
5 \\
\text { Overall }\end{array}$ & Full scale & Full scale & $\begin{array}{l}-.03 \\
-.02 \\
.03 \\
-.13 \\
-.04 \\
\end{array}$ & $\begin{array}{l}-.11 \\
-.05 \\
-.26 \\
-.28 \\
-.16 \\
\end{array}$ & $\begin{array}{l}.01 \\
-.004 \\
.05 \\
.13\end{array}$ \\
\hline Wilson (2016) & 1 & Full scale & Full scale & -.14 & -.25 & .12 \\
\hline Wilson (2017) & 5 & Full scale & Full scale & -.14 & -.36 & .20 \\
\hline Wilson (2005b) & $\begin{array}{l}4 \\
5 \\
\text { Overall }\end{array}$ & Full scale & Full scale & $\begin{array}{l}-.16 \\
-.02 \\
-.12 \\
\end{array}$ & $\begin{array}{l}-.30 \\
-.17 \\
-.27 \\
\end{array}$ & $\begin{array}{l}.17 \\
.02\end{array}$ \\
\hline Wilson (2005c) & 4 & Full scale & Full scale & -.08 & -.34 & .15 \\
\hline Wilson (2005d) & $\begin{array}{l}4 \\
5 \\
\text { Overall }\end{array}$ & $\begin{array}{l}\text { Full scale } \\
\text { Full scale }\end{array}$ & $\begin{array}{l}\text { Full scale } \\
\text { Full scale }\end{array}$ & $\begin{array}{l}-.21 \\
-.31 \\
-.24\end{array}$ & $\begin{array}{l}-.13 \\
.02 \\
-.09\end{array}$ & $\begin{array}{l}.10 \\
.09\end{array}$ \\
\hline
\end{tabular}

Note. The measures of environmentalism included are coded such that $1=$ climate change belief, $2=$ support for action, $4=$ Green politics, $5=$ pro-environmental attitudes 


\section{Appendix C}

\section{Replication of Study $3 \mathrm{~B}$ and $3 \mathrm{C}$ using shortened $\mathrm{SDO}_{7}$ scale}

In Chapter 4, I show that the 16-item $\mathrm{SDO}_{7}$ scale reliably separates into two subscales to measure SDO-D and SDO-E. To examine whether the scale could also be used in a shortened 8-item version, I then replicated the analyses presented in Studies 3B and 3C with the shortened version.

Firstly, Table C presents the CFAs on the unidimensional, two-factor, and four-factor models based on the shortened scale. Replicating the findings of the full scale, these support the two-dimensional model providing superior fit compared to the one-dimensional model (Study 3B: $\left(\Delta \chi^{2}(1)=17.86, p<.001 ;\right.$ Study 3C: $\left.\Delta \chi^{2}(1)=6.76, p=.009\right)$.

Finally, examination of the Cronbach's alphas for each subscale showed they were reasonably reliable (for shortened scales) in Study 3B (SDO-D: $\alpha=.69$, SDO-E: $\alpha=.75$ ) and $3 \mathrm{C}$ (both SDO-D and SDO-E: $\alpha=.67$ ). 


\section{Table C.}

Model fit for one- and two-dimension models, two-method models, and four-factor models using shortened scales from in Studies $3 B$ and $3 C$.

\begin{tabular}{lcccccccc}
\hline & $\boldsymbol{X}^{2}$ & $d f$ & $\boldsymbol{X}^{2} / d f$ & CFI & AIC & RMSEA & $\begin{array}{c}\text { 90\% CI } \\
\text { RMSEA }\end{array}$ & SRMR \\
\hline \hline One-dimension model & 66.93 & 20 & 3.35 & .94 & $13,312.10$ & .07 & {$[.05, .09]$} & .04 \\
Two-dimension model & 57.28 & 19 & 3.01 & .95 & $13,298.67$ & .06 & {$[.05, .08]$} & .04 \\
Two-method model & 38.64 & 9 & 4.29 & .97 & $13,272.59$ & .05 & {$[.02, .07]$} & .03 \\
Four-factor model & 3.25 & 10 & .33 & 1.00 & $13,241.98$ & .00 & {$[.00, .00]$} & .01
\end{tabular}

Study 3 T1

\begin{tabular}{lcccccccc}
\hline One-dimension model & 79.89 & 20 & 3.99 & .93 & $18,466.59$ & .07 & {$[.05, .08]$} & .04 \\
Two-dimension model & 72.93 & 19 & 3.84 & .94 & $18,459.18$ & .07 & {$[.05, .08]$} & .04 \\
Two-method model & 52.59 & 19 & 2.77 & .96 & $18,432.29$ & .05 & {$[.04, .07]$} & .03 \\
Four-factor model & 19.47 & 10 & 1.95 & .99 & $18,401.77$ & .04 & {$[.01, .06]$} & .02 \\
\hline
\end{tabular}

Notes. $\chi^{2}=$ Chi Square, $d f=$ degrees of freedom, CFI = comparative fit index, RMSEA = root mean square approximation, 90\% CI RMSEA $=$ confidence interval around RMSEA of the change in fit between models, AIC = Akaike Information Criterion, SRMR = standardized root mean square residual. 


\section{Appendix D}

\section{Interview schedule used in Study 4}

Firstly, have the participant read the information sheet, give them an opportunity to ask questions, and then give them the consent form to sign.

\section{Introduction}

Thanks for agreeing to take part in our study today. As you will have read from the information form, in this study we are really interested in getting your honest opinions on some social and political issues. We have a few issues that we are interested in, but we may not get to all of them today. We will start with some general questions. We whatare interested in your opinions - there are no right or wrong answers to these questions, we just want your thoughts on them.

\section{Section 1. General questions}

Ask each of the following questions, and after each ask follow up questions

1. What do you think some of the biggest problems facing New Zealand today are?

2. What do you think some of the biggest problems facing the world today are?

3. What do you think some of the biggest problems facing New Zealand in the future will be?

4. What do you think some of the biggest problems facing the world in the future will be?

Follow up questions:

1. Why is [that issue] a problem?

2. What are some solutions to [that problem]? Why?

3. What are some of the barriers that need to be overcome to solve [the problem]? Why?

4. What do you think the Government should do about [the problem]? Why?

5. What do you think individuals should do about [the problem]? Why?

6. What do you think businesses should do about [the problem]? Why?

Thank you for answering these questions 
Next, lead into whichever topics flows best from the answers given in section 1. The idea here is to obscure the focus of the study (the environment).

- If climate change is mentioned as a problem, go to section 2

- If race relations/inequality is mentioned, go to section 3, then to section 2 after

- If employment is mentioned, go to section 4, then to section 2 after

- If none of these are mentioned, go to either section 3 or 4 so that the focus (environment) is less clear, then to section 2 after

Once the Environmental topic has been discussed, end the interview.

Show them this graph, introducing it as results of a previous study of New Zealanders that asked what the biggest problem facing New Zealand today was, and explain that one important issue was [next section topic]

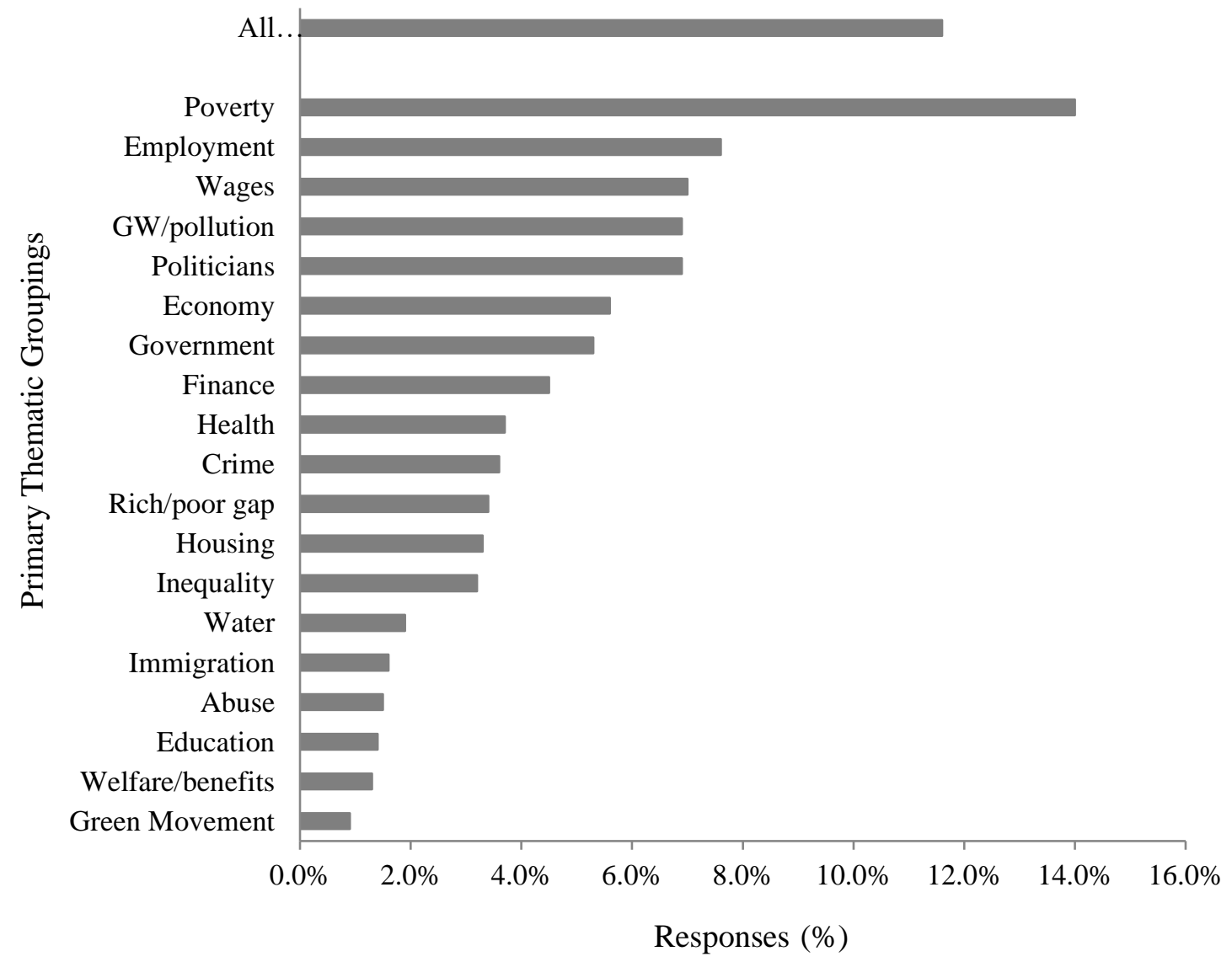

Thematically grouped responses (\%) to the question: "What do you think is the most important problem facing New Zealand today" 


\section{Section 2. Environment topic}

Research on New Zealander's opinions recently found that climate change was a problem that many people relate to.

1. What are your views on climate change?

2. Do you think climate change is a problem?

3. What are some solutions to climate change? Why?

4. What are some of the barriers that need to be overcome to solve climate change? Why?

5. What do you think the Government should do about climate change? Why?

6. What do you think individuals should do about climate change? Why?

7. What do you think businesses should do about climate change? Why?

Some people are willing to make sacrifices to their standard of living, for example accepting higher prices, drive less, or conserve energy, in order to protect the environment.

1. What are your thoughts on this? Why?

2. To what extent are you willing to make sacrifices for the environment?

3. What are some of the barriers that stop you from doing more for the environment?

The Government has looked into the feasibility of deep sea drilling under the sea bed.

1. What do you think about this? Why?

2. Who should benefit from this if it does go ahead? (second prompt: e.g. profits to investors or community) Why?

\section{Section 3. Race relations topic}

Another common answer about the biggest problem in New Zealand was inequalitymeaning that not all groups have the same opportunities. 
1. What are your views on this? Why?

2. Why is/isn't inequality a problem?

3. What are some solutions to inequality? Why?

4. What are some of the barriers that need to be overcome to solve inequality? Why?

5. What do you think the Government should do about inequality? Why?

6. What do you think individuals should do about inequality? Why?

7. What do you think businesses should do about inequality? Why?

One solution to racial inequality is affirmative action policies. These policies redistribute resources to minority groups.

1. What do you think about these policies? Why?

2. Are you supportive of these policies? Why/why not?

3. Do you think resources/jobs etc. need to be redistributed in favour of disadvantaged groups?

\section{Section 4. Economy}

Another issue that ranked highly among New Zealander's responses was the economy.

1. What are your views on this? Why?

2. Why is/isn't the economy a problem?

3. What are some solutions to this problem? Why?

4. What are some of the barriers that need to be overcome to solve the problem? Why?

5. What do you think the Government should do about the economy? Why?

6. What do you think individuals should do about economy? Why?

7. What do you think businesses should do about economy? Why? 


\section{Conclusion}

Thank you for answering these questions. This is the end of the interview. Once we are finished with all of the interviews, we will email you a debriefing to let you know more information about the study.

\section{Note:}

The actual interview schedule may differ slightly from this, but no additional questions will be any more intrusive than those above. 